\title{
Synchronisation chaotisch fluktuierender Halbleiterlaser
}

\author{
Dissertation \\ zur Erlangung des Doktorgrades der \\ Mathematisch-Naturwissenschaftlichen Fakultäten der \\ Georg-August-Universität zu Göttingen
}

\author{
vorgelegt von \\ Immo Wedekind \\ geboren in \\ Köln
}

Göttingen 2005 
D7

Referent: $\quad$ Prof. Dr. Ulrich Parlitz Korreferent: $\quad$ Prof. Dr. Dirk Ronneberger Tag der mündlichen Prüfung: 26. April 2005 


\section{Zusammenfassung}

Die vorliegende Arbeit beschäftigt sich mit der experimentellen und numerischen Synchronisation zweier optisch unidirektional gekoppelter Halbleiterlaser. Bei unidirektionaler Kopplung werden die beiden Systeme als treibendes und als empfangendes System entsprechend der Kopplungsrichtung bezeichnet. Schwerpunkt dieser Untersuchung liegt in der Synchronisation der Dynamik des optisch rückgekoppelten Halbleiterlasers als treibendes System. Die Synchronisation der Dynamik der Intensitätseinbrüche (low frequency fluctuations - LFFs), der Intensitätssprünge (low frequency power jump-ups - LFJs) und die Dynamik des Kohärenzkollapses wird untersucht. Gemäß dem allgemein akzeptierten deterministischen Modell von LANG und KOBAYASHI handelt es sich bei diesen Intensitätsfluktuationenen um extrem hochdimensionales Chaos.

Die langsame Änderung der Hüllkurve der Gesamtintensität beider Laser bei LFFbzw. LFJ-Dynamik ist im Experiment mit zeitgemäßer Laborausstattung gut detektierbar. Im Experiment wird in beiden Fällen sowohl Synchronisation als auch Antisynchronisation erreicht. Die detaillierten Betrachtungen der Synchronisation für die Variation der wichtigsten Parameter zeigen charakteristische Wiederholungen der Synchronisationsbereiche. Die Synchronisationsbereiche liegt in direkter Nachbarschaft zu Bereichen der Antisynchronisation.

Neben diesen Untersuchungen der Gesamtintensität werden umfangreiche Messung des optischen Leistungsspektrums beider Laser während der Synchronisation gezeigt. Diese Messungen enthüllen viele aktive longitudinale Lasermoden in beiden Lasern. Die Veränderung der Lichtfrequenzen dieser Moden erklärt auf einfache Weise das wiederholte Auftreten der Synchronisationsbereiche in den untersuchten Parameterbereichen. Mit einem Monochromator ist es experimentell möglich, die einzelnen Modenintensitäten beider Laser zeitgleich aufzunehmen und auf Synchronisation zu untersuchen. Speziell im Vergleich zu der zur selben Zeit aufgezeichneten Gesamtintensität beider Laser ergeben sich überraschende Ergebnisse. Es zeigt sich Modensynchronisation bei zeitgleicher Antisynchronisation der Gesamtintensität. Die Gesamtintensität der extrem schnellen Dynamik des Kohärenzkollapses konnte experimentell nicht aufge- 
nommen werden, hingegen ist es problemlos möglich die deutlich langsamer fluktuierenden einzelnen Modenintensitäten aufzunehmen. Modensynchronisation kann für bestimmte Parameterbereiche zwischen beiden Lasern nachgewiesen werden. Ohne optische Rückkopplung des treibenden Lasers zeigt sich normalerweise keine Dynamik der Gesamtintensität. Durch den Monochromator kann jedoch festgestellt werden, dass an bestimmtem Parameterkombinationen spontane Modensprünge auftreten, die auf die Gesamtintensität praktisch keinen Einfluss haben. Auch diese Modensprünge sind zwischen beiden Lasern sowohl synchronisierbar als auch antisynchronisierbar.

Im numerischen Teil dieser Arbeit wird das bekannte Lang-Kobayashi-Modell geeignet erweitert, um die wenig beachtete LFJ-Dynamik simulieren zu können. Ermöglicht wird dies durch die Berücksichtigung von Mehrfachreflexionen im externen Resonator. Desweiteren wird die Frequenzverstimmung zwischen beiden Lasern in dem Modell berücksichtigt und mehrere longitudinale Moden eingeführt. Zur Nachbildung des experimentellen Aufbaus wird ein so erweitertes Lang-Kobayashi-Modell mit dem eines multimodalen Halbleiterlasers gekoppelt. Der Übergang von Synchronisation zu Antisynchronisation stimmt in der numerischen Simulation sehr gut mit den experimentellen Daten überein. Dies gilt sowohl für die LFF- als auch für die LFJ-Dynamik. Die Modensynchronisation wird von diesem Modell ebenfalls gut wiedergegeben, auch im Bereich des Kohärenzkollapses. Auch der überraschende Fall von Modensynchronisation bei zeitgleicher Antisynchronisation der Gesamtintensität lässt sich in der Simulation nachvollziehen. Über die Bestätigung des experimentellen Ergebnisse hinaus, ist es mit diesem Modell möglich, die Synchronisation auch auf wesentlichen schnelleren, dem Experiment nicht zugänglichen Zeitskalen zu analysieren. 


\section{Inhaltsverzeichnis}

1 Einleitung 1

1.1 Aufbau der Arbeit . . . . . . . . . . . . . . . . . . . . . 2

2 Der Halbleiterlasen 5

2.1 Funktionsweise . . . . . . . . . . . . . . . . . . . 6

2.2 Kantenemittierender Halbleiterlaser . . . . . . . . . . . . . . . . . . . 9

2.3 Oberflächenemittierender Halbleiterlasen . . . . . . . . . . . . . . . . . 10

2.4 Semiklassische Lasergleichungen . . . . . . . . . . . . . . . . . . . . . . 12

2.5 Multi-Longitudinale Lasergleichungen . . . . . . . . . . . . . . . . . 16

2.6 Modensprünge . . . . . . . . . . . . . . . . . . . . . . . . . . . . 17

3 Halbleiterlaser mit optischer Rückkopplung 19

3.1 Low Frequencv Fluctuations . . . . . . . . . . . . . . . . . . . . 20

3.2 Die Lang-Kobavashi-Modellgleichungen . . . . . . . . . . . . . . . . . . 22

3.3 Fixpunkte und Stabilität . . . . . . . . . . . . . . . . . . . . . . . . 24

3.4 Multimode Lang-Kobavashi-Modelloleichungen . . . . . . . . . . . . . . 26

3.5 Modellierung der Dvnamik der Intensitätssprünge . . . . . . . . . . . . 29

4 Synchronisation 33

4.1 Kopplung . . . . . . . . . . . . . . . . . . . . . . . . . . . . . . . . . . . . . 34

4.2 Svnchronisationsarten . . . . . . . . . . . . . . . . . . 35

4.2 .1 Identische Svnchronisation . . . . . . . . . . . . . . . . . 35

4.2 .2 Antisvnchronisation . . . . . . . . . . . . . . . . . 36

4.2 .3 Phasensvnchronisation . . . . . . . . . . . . . . 36

4.3 Stabilität . . . . . . . . . . . . . . . . . 37

4.4 Messung der Svnchronisation . . . . . . . . . . . . . . . . . . . . . . . 39

4.4 .1 Messung der identischen Svnchronisation . . . . . . . . . . . . . 39

4.4 .2 Messung der Antisvnchronisation . . . . . . . . . . . . . . . . . 41

4.4 .3 Messung der Phasensvnchronisation . . . . . . . . . . . . . . . . 42 
5 Experimentelle Svnchronisation von Lasern 45

5.1 Historischer Überblick . . . . . . . . . . . . . . . . . . . . . . . . 45

5.2 Experimenteller Aufbaul . . . . . . . . . . . . . . . . . . . . 46

5.3 Svnchronisation und Antisvnchronisation der LFF-Dvnamik . . . . . 48

5.3 .1 Einfluss der Kopplungsstärke . . . . . . . . . . . . . . . 49

5.3 .2 Übergang von Svnchronisation zu Antisvnchronisation ..... 53

5.3.3 Parameterbereiche der Svnchronisation und Antisvnchronisation 60

5.3.4 Messung des optischen Leistungspektrums . . . . . . . . . . . 62

5.3 .5 Modensunchronisation ... . . . . . . . . . . 68

5.4 Svnchronisation und Antisvnchronisation der LFJ-Dvnamik $\ldots . .73$

5.4 .1 Einfluss der Kopplungsstärke . . . . . . . . . . . . 74

5.4.2 Parameterbereiche der Svnchronisation und Antisynchronisation 74

5.4 .3 Messung des optischen Leistungsspektrums . . . . . . . . . . . 77

5.4 .4 Modensunchronisation . . . . . . . . . . . . . . . . 80

5.5 Modensvnchronisation im Kohärenzkollaps . . . . . . . . . . . . 83

5.6 Svnchronisation der spontanen Modensprünge . . . . . . . . . . . . 83

5.7 Antisvnchronisation der spontanen Modensprünge . . . . . . . . . 86

6 Numerische Simulation der Lasersynchronisation 91

6.1 Historischer Überblick . . . . . . . . . . . . . . . . . . . . . . . 91

6.2 Modellierung der Wellenlängenabhängigkeit . . . . . . . . . . . . 92

6.3 Numerisches Svnchronisationschema . . . . . . . . . . . . . . . . 95

6.4 Svnchronisation und Antisvnchronisation der LFF-Dvnamik . . . . 97

6.4.1 Parameterbereiche der Svnchronisation und Antisvnchronisation 98

6.4.2 Übergang zwischen Svnchronisation und Antisvnchronisation . . 104

6.5 Svnchronisation und Antisvnchronisation der LFJ-Dvnamik . . . . . 104

6.6 Modensvnchronisation . . . . . . . . . . . . . . . . . . . . 108

6.7 Puls- und Phasenraumdvnamik . . . . . . . . . . . . . . 118

$\begin{array}{lll}7 & \text { Diskussion und Ausblick } & 125\end{array}$

\begin{tabular}{ll}
\hline A Symbolverzeichnis & 129
\end{tabular}

B Komponenten des experimentellen Aufbaus 133

\begin{tabular}{ll}
\hline C Farbtabellen & 135
\end{tabular}

\begin{tabular}{ll}
\hline P Parameter der numerischen Simulation & 139
\end{tabular} 


\section{Kapitel 1}

\section{Einleitung}

Die Synchronisation zweier chaotischer Systeme ist ein sehr faszinierendes Phänomen. Nur durch eine schwache Kopplung verbunden, können zwei identische, chaotisch schwingende Systeme bereits nach kurzer Zeit eine identische Dynamik beschreiben. Und dies funktioniert trotz der bekannten empfindlichen Abhängigkeit von den Anfangsbedingungen bei diesen Systemen. Neben dieser identischen Synchronisation werden heute viele verschiedene Arten von Synchronisation chaotischer Systeme unterschieden, die sehr unterschiedliche Anforderungen an die Systeme und Kopplung haben.

So faszinierend die Entdeckung dieses Phänomens durch FuJISAKA und Yamada 1983 [21] am Beispiel des Lorenz-Systems war, so inspirierend war die Aussicht einer praktischen Anwendung, die PECORA und CARROL 1990 [53] vorschlugen: die sichere Kommunikation mittels solcher Systeme. Besteht zwischen zwei synchronisierten, chaotischen Systemen eine unidirektionale Kopplung, so wirkt eines der Systeme als treibendes System oder Sender und das andere als Empfangssystem. Die Sicherheit soll hier durch die empfindliche Abhängigkeit von den Parametern entstehen, nur eine leichte Verstimmung der Parameter im Sende- oder im Empfangssystem würde die Synchronisationsqualität empfindlich stören. Dementsprechend wären diese Parameter der Schlüssel dieser Kommunikation. Je komplexer das dynamische System, desto schwieriger wäre die Analyse und demnach die Rekonstruktion dieser Parameter aus dem übermittelten Signal.

Durch diese Motivation beflügelt wurde die Synchronisation auch an sehr schnellen und komplexen System numerisch wie auch experimentell untersucht, speziell auch für den Fall unidirektionaler Kopplung. Ein Beispiel für ein solches System ist der optisch rückgekoppelte Halbleiterlaser, um den es in dieser Arbeit geht. Die entstehende Dynamik dieses sehr einfachen Aufbaus aus Laserdiode und in einer Entfernung von 10-100cm stehenden Reflektors ist äußerst vielfältig, komplex und extrem schnell. Geht 
man von dem deterministischen Modell eines solchen Systems von LANG und KoBAYASHI [41] aus, konnten AHLERS et al. [5] in numerischen Rechnungen über 100 positive Lyapunovexponenten bestimmen. Die extrem schnelle Dynamik in der Größenordnung einiger Gigahertz überfordert auch heute noch die gängige Ausstattung der meisten Labore. Die Hüllkurve dieser schnellen Dynamik zeigt unter bestimmten Bedingungen jedoch auch eine mit 3-30Mhz verhältnismäßig langsame Dynamik, die s.g. low frequency fluctuations kurz LFFs. Es zeigen sich gut zu beobachtende, sich wiederholende Intensitätseinbrüche des Lasers.

Im Laufe der neunziger Jahre des letzten Jahrhunderts konnte zuerst numerisch die identische Synchronisation der LFFs gezeigt werden. Etwas später gelang auch die experimentelle Synchronisation dieser Dynamik. Zu dieser Zeit begann ich mit meiner Diplomarbeit zur experimentellen Synchronisation dieser Dynamik. Überraschenderweise war in meinem Experiment nicht nur identische Synchronisation sondern auch Antisynchronisation - der empfangende Laser reagiert mit einem Intensitätssprung auf den Intensitätseinbruch des treibenden Lasers - der LFF-Dynamik zu beobachten. Nach dem Abschluss dieser experimentellen Arbeit, war noch kein Modell für dieses Phänomen gefunden. Kurze Zeit nach dieser Entdeckung wurde auch von anderen Arbeitsgruppen diese Synchronisationsart experimentell beschrieben.

In dieser Arbeit habe ich die experimentellen Untersuchung sehr erweitert. Systematisch werden die Laserparameter auf Synchronisation und Antisynchronisation beider Laser untersucht. Als weiterer Untersuchungsschwerpunkt wird das Leistungsspektrum des Laserlichtes systematisch analysiert. Neben der bekannten LFF-Dynamik existiert noch eine sehr eng verwandte, aber in der Literatur kaum beachtete Dynamik eines solchen Systems, die statt den bekannten Intensitätseinbrüchen Intensitätssprünge aufweist. Auch für diese Dynamik werden die Laserparameter systematisch auf Synchronisation bzw. Antisynchronisation untersucht. Darüber hinaus wird das bekannte Lang-Kobayashi-Modell geeignet erweitert und an die experimentellen Gegebenheiten angepasst, um ein qualitatives Modell zur Beschreibung der Dynamik möglichst aller experimentell entdeckten Phänomene zu erreichen.

\subsection{Aufbau der Arbeit}

In Kapitel 2 beschreibe ich kurz die historische Entwicklung des Halbleiterlasers. Danach wird die Herleitung der wichtigen semiklassischen Lasergleichungen für die Beschreibung der Dynamik dieses Lasertyps dargestellt. Kapitel 3 beschreibt die Entwicklung der optische rückgekoppelten Halbleiterlaser mit anschließender Diskussion der LFF-Dynamik anhand der Literatur. Danach beschreibe ich das Modell von LANG 
und Kobayashi mit anschließender Untersuchung der Stabilität und Erklärung der LFF-Dynamik. Zum Schluss dieses Kapitels gehe ich auf einige wichtige Erweiterungen dieses numerischen Modells ein. Zum einen wird die Erweiterung auf mehrere longitudinale Lasermoden beschrieben, zum anderen die Einführung von Mehrfachreflexionen im externen Resonator zur Beschreibung der Dynamik der Intensitätssprünge.

Kapitel 4 führt die Theorie der Synchronisation nichtlinearer, chaotischer Systeme ein. Es wird auf die Kopplung und die wichtigsten Synchronisationsarten eingegangen. Danach werden Methoden zu Detektion dieser Synchronisationsarten im Experiment beschrieben, wie sie in den von mir durchgeführten Experimenten Anwendung fanden.

Alle experimentellen Ergebnisse sind in Kapitel 5 zusammengefasst. Zu Anfang werden systematisch Untersuchungen auf Synchronisation und Antisynchronisation für unterschiedliche Kombinationen von Temperatur, Pumpstrom und Kopplungsstärke gezeigt. Im weiteren folgen Messungen zum Übergang zwischen Synchronisation und Antisynchronisation. Danach wurde mittels Spektrometer das Leistungspektrum beider Laser aufgezeichnet. Zum Schluss werden umfangreiche Messungen zur Modensynchronisation sowohl für die Dynamik der Intensitätseinbrüche und Intensitätssprünge als auch für den Parameterbereich des Kohährenzkollaps und der spontanen Modensprünge präsentiert.

Das 6. Kapitel beschreibt das verwendete numerische Synchronisationsschema. Es werden die Ergebnisse der Simulationen denen der experimentellen Messungen gegenübergestellt. 



\section{Kapitel 2}

\section{Der Halbleiterlaser}

Eine der grundlegenden theoretischen Vorarbeiten zur Entwicklung Lasers war ein Postulat von Einstein 1917. Einstein nahm an, dass es für die Emission von Lichtquanten eines Atoms zwei unterschiedliche Mechanismen geben müsse, die spontane und die induzierte Emission. Im Gegensatz zur spontanen Emission, die bei jeder Art von Lichterzeugung stattfindet, gelang ein experimenteller Nachweis der induzierten Emission erst elf Jahre später durch LADENBURG bei der Untersuchung von elektrischen Entladungen in einem mit Neon gefüllten Rohr. Im Jahre 1954 konnte TownES et. al. 24] erstmals experimentell zeigen, dass das heute bekannte Konzept des Lasers aus den drei Grundbestandteilen - Pumpe, laseraktives Material und Resonator - funktioniert, damals jedoch noch im Mikrowellenbereich. Sinngemäß nannte Townes das Gerät auch MASER für Microwave Amplification by Stimulated Emission of Radiation. Das dieses Prinzip auch im optischen Frequenzbereich möglich ist konnte Maimann 1960 mit dem ersten Rubin-Lasen 1 zeigen. Dieser noch im Pulsbetrieb arbeitenden Laser führte in den folgenden Jahren zu einer enormen Forschungstätigkeit auf diesem Gebiet. Noch im selben Jahr konnte der Helium-Neon-Laser als erster cw-Lasen 2 vorgestellt werden, er emittierte ungepulst kohärentes, rotes Laserlicht.

1961 wurde erstmals die Aussendung von inkohärentem Licht - im infraroten Wellenlängenbereich - aus einer Halbleiterdiode beobachtet. Bereits ein Jahr später konnte HALL et. al. [27] den ersten infraroten Halbleiterlaser herstellen, basierend auf einem Gallium-Arsenid p-n-Übergang. Nur wenige Wochen später zeigte HolONyAK [31] einen roten Halbleiterlaser basierend auf einem zur damaligen Zeit neuartigen GaAsP p-n-Übergang.

\footnotetext{
${ }^{1}$ LASER - Light Amplification by Stimulated Emission of Radiation

2 constant wave laser - Dauerstrichlaser
} 


\subsection{Funktionsweise}

Bei allen Halbleiterlasern wird ein p-n-Übergang sowohl als laseraktives Material als auch als Pumpe verwendet, häufig wird dieser Lasertyp deshalb auch Diodenlaser genannt. Steht ein Halbleiter im thermischen Gleichgewicht ist das Valenzband mit Elektronen gefüllt und das Leitungsband ist leer. Die Fermi-Energie $\mu$, über die mit der Fermi-Dirac-Verteilung

$$
f(E)=\frac{1}{e^{\frac{E-\mu}{k_{B} T}}+1}
$$

die Besetzungswahrscheinlichkeit einzelner Energieniveaus durch Elektronen beschrieben wird, liegt dann genau zwischen dem Valenzband und dem Leitungsband, es existieren keine freien Ladungsträger. Bei nicht verschwindender Temperatur $T$ gibt Gleichung (2.1) die Aufenthaltswahrscheinlichkeit von Ladungsträgern im Leitungsband (Elektronen) und im Valenzband („Löchern“) an. Die Lichterzeugung in einem Halbleiter, spontan oder induziert, entsteht durch Rekombination von LeitungsbandElektronen mit Valenzband-Löchern. Strahlung kann hierbei jedoch nur dann entstehen, wenn der Wellenzahlvektor $\boldsymbol{k}$ des Elektrons erhalten bleibt. Nur in direkten Halbleitern ist dies für die überwiegende Anzahl von Rekombinationen möglich. Aus diesem Grund sind praktisch alle Halbleiterlaser aus III-V-Verbindungen (z.B. GalliumArsenid GaAs) hergestellt, da nur diese Stoffe gleichzeitig direkte Halbleiter sind und eine Bandlücke entsprechend dem optisch sichtbaren Bereich besitzen.

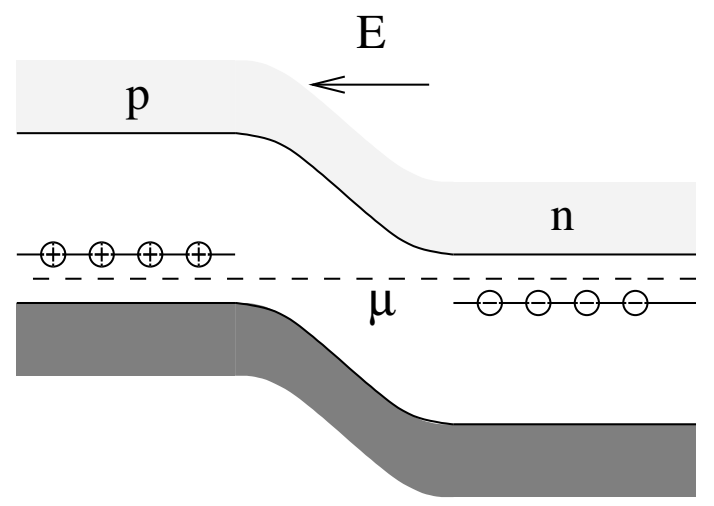

Abbildung 2.1: Der p-n-Übergang im thermischen Gleichgewicht.

Ähnlich wie bei anderen Lasertypen muss auch bei dem Diodenlaser eine Ladungsträgerinversion vorliegen, bevor Laseraktivität entstehen kann. Dies geschieht durch die Dotierung in Verbindung mit dem p-n-Übergang. Durch die p-Dotierung werden 
zusätzliche, leere Energieniveaus knapp oberhalb des Valenzbandes erzeugt. Die nDotierung fügt zusätzliche, besetzte Energieniveaus knapp unterhalb des Leitungsbandes hinzu. Durch Zusammenfügen des p- und des n-dotierten Material entsteht ein p-n-Übergang mit einer in Abbildung 2.2 skizzierten Bandstruktur.

Durch Anlegen eines externen elektrischen Feldes $E_{\text {ext }}$, welches stärker ist, als das durch Ladungsträgerdiffusion entstandene interne elektrische Feld $E$ entsteht ein Stromfluss durch den p-n-Übergang. Bei der Rekombination der Ladungsträger im Bereich der Grenzschicht kommt es bei direkten Halbleitern zu spontaner und induzierter Emission.

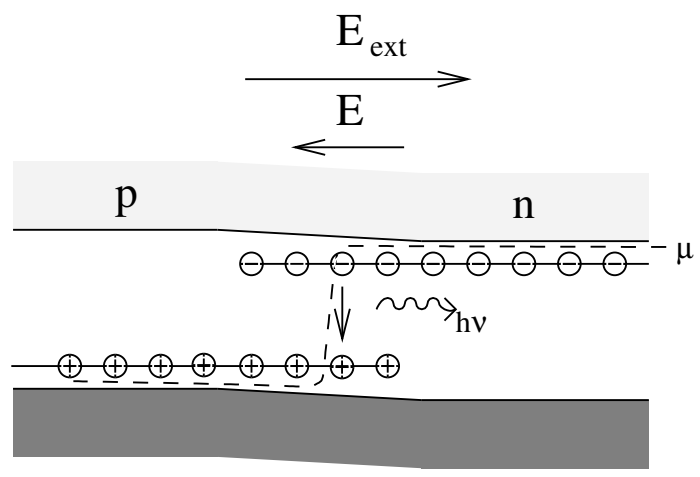

Abbildung 2.2: Stromfluss durch den p-n-Übergang.

Zu einem kompletten Laser fehlt noch der Resonator, der im einfachsten Fall durch das Polieren der Endflächen des Halbleiterkristalls als Fabry-Perot-Typ ausgeführt werden kann.

Dieser so konstruierte homojunction semiconductor laser besitzt nur eine sehr geringe Effizienz und Haltbarkeit, da nur in dem räumlich sehr kleinen Bereich der Grenzschicht eine Ladungsträgerinversion vorhanden ist. Nur durch sehr hohe Ströme, die schnell das Halbleitermaterial degenerierten, ist Laseraktivität bei sehr geringen Arbeitstemperaturen - weit unterhalb der Raumtemperatur - möglich.

Eine enorme Effizienzsteigerung konnte durch das Einbringen einer speziell dotierten Zusatzschicht aus einem anderen Halbleitermaterial mit geringerer Bandlücke zwischen das p- und das n-Material erzielt werden. Diese Heterostrukturen - die Verbindung von unterschiedlichen Halbleitermaterialien - wurde von Alferov [6] und Kroemer [40] 1963 unabhängig voneinander für die Verwendung in Diodenlasern vorgeschlagen 3 . 1970 konnte AlfEROV erstmals einen so konstruierten Laser demonstrieren, der bei Raumtemperatur arbeitete.

\footnotetext{
${ }^{3}$ Alerov und Kroemer erhielten im Jahr 2000 zusammen mit Kilby den Nobelpreis für Physik u.a. für die Entwicklung dieser Heterostrukturen.
} 


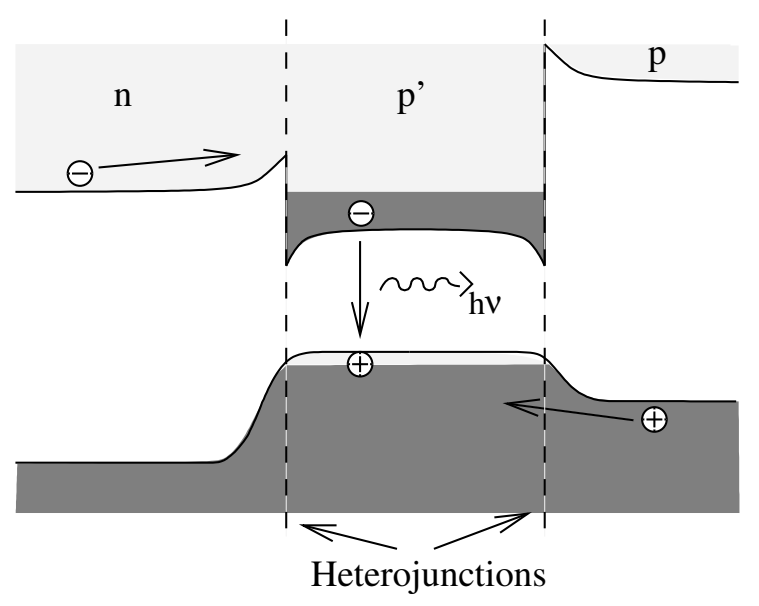

Abbildung 2.3: Bandstruktur des Heterolasers.

Die Effizienzsteigerung bei diesen Heterolasern (aus dem englischen heterojunction semiconductor laser) lässt sich darauf zurückführen, dass bei geeigneter Vorspannung der gesamte räumliche Bereich des zusätzlich eingebrachten Halbleiters in den Zustand der Ladungsträgerinversion versetzt werden kann (Abb. 2.3).

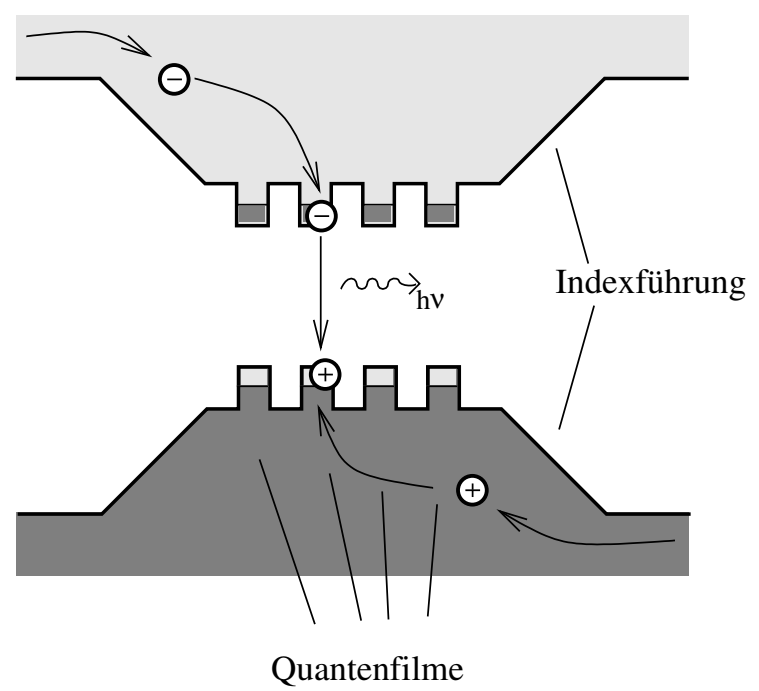

Abbildung 2.4: Bandstruktur eines indexgeführten MQW-Lasers.

Die konsequente Weiterentwicklung dieser Technologie führte später zu den Quantenfilm-Lasern (engl. quantum well laser) und Mehrfach-Quantenfilm-Lasern (engl. multiple quantum well laser MQW) [76]. Ein Quantenfilm-Laser ist im Prinzip ein Heterolaser bei dem die Dicke der zusätzlich eingebrachten Halbleiterschicht sehr klein ist. Auf Grund der geringen Dicke werden Quanteneffekte für die Elektronen in diesem Bereich wirksam und diskretisieren deren Energie in einer Raumrichtung. Zum 
einen lässt sich bei solchen Lasern durch die Schichtdicke sehr genau die Bandlücke definieren, zum anderen können mehr Elektronen an strahlenden Rekombinationsprozessen teilnehmen, die Effizienz steigt und die Laserschwelle sinkt [3]. In einem Mehrfach-Quantenfilm-Laser werden in exakt definierten Abständen mehrere Quantenfilme verwendet (Abb. 2.4). Praktisch alle momentan kommerziell verfügbaren Diodenlaser sind MQW-Laser.

Die weitere Entwicklung führte zu den Quantendraht- und Quantenpunkt-Laser, bei denen die Elektronen in quasi eindimensionalen bzw. nulldimensionalen Bereichen konzentriert werden. Sie zeigen vor allen eine sehr geringe Laserschwelle, geringe Temperaturabhängigkeit, hohe Modulationsbandbreite und geringere Linienbreite [25]. Für Hochleistungsanwendungen wurde jüngst der Quanten-Kaskaden-Laser entwickelt. Durch geschickte Wahl der Materialien wird bei diesem Lasertyp die Frequenz des emittierten Lichtes nicht mehr durch die Größe der Bandlücke definiert, sondern durch die diskreten Zustände in den Quantenfilmen, die durch den Herstellungsprozess variiert werden können. Durch diese exakte Kontrolle der Elektronenzustände ist es möglich nicht nur eine Energiestufe zur Lichtgewinnung zu verwenden sondern mehrere äquidistante Energiestufen. Die Elektronen durchlaufen also eine Kaskade von Energiestufen. Die Effizienz steigt hierbei enorm und ermöglicht extrem leistungsfähige Halbleiterlaser[18].

\subsection{Kantenemittierender Halbleiterlaser}

In kantenemittierenden Halbleiterlasern (EELs - edge emitting semiconductor lasers) wird das Licht innerhalb der laseraktiven Schicht wie in einem rechteckigen optischen Wellenleiter geführt (siehe Abb. 2.5). Die Begrenzung dieses Wellenleiters werden in der Richtung senkrecht zur laseraktiven Schicht durch den geringeren Brechungsindex der beiden p- und n-dotierten Halbleiter erreicht. Ohne weitere Maßnahmen findet eine Eingrenzung des elektromagnetischen Feldes in der Ebene der laseraktiven Schicht lediglich über die Stärke des durch diese Schicht fließenden Stromes statt. Durch Protonen-Implantation in den Außenbereich dieser Halbleiterschicht wird der Widerstand erhöht, um den Stromfluss einzugrenzen. So konstruierte Laser werden als gewinngeführt (engl. gain-guided) bezeichnet. Eine andere Methode ist die räumliche Veränderung des Brechungsindexes auch innerhalb der Ebene der laseraktiven Schicht, diese Laser sind indexgeführt (engl. index-guided). Augenfälligster Unterschied ist die für letzteren Laser wesentlich geringeren Schwell-Strom bei höheren Herstellungskosten. Die meisten kommerziellen Laser sind heutzutage indexgeführt.

Der Resonator kann im einfachsten Fall durch die parallelen, polierten Facetten des 
Halbleiterkristalls im Sinne eines Fabry-Perot-Resonators gebildet werden. Übliche Resonatorlängen von EELs liegen bei $L \approx 300 \mu$ m mit einer Facetten-Reflektivität von $R \approx 0.3$. Durch den geringen longitudinalen Modenabstand von nur $\Delta \nu_{L} \approx 100 \mathrm{GHz}$ fallen sehr viele dieser Moden in den Verstärkungsbereich des laseraktiven Materials. Kantenemittierende Laser sind im Allgemeinen also Multi-Mode-Laser.

Durch Verwendung von wellenlängenabhängigen Reflektoren durch Einbringung optischer Indexgitter in oder an den Enden der laseraktiven Schicht (DFB - distributed feedback bzw. DBR - distributed bragg reflector) können auch Single-Mode-Laser hergestellt werden, die speziell in der optischen Datenübertragung wichtig sind [3].

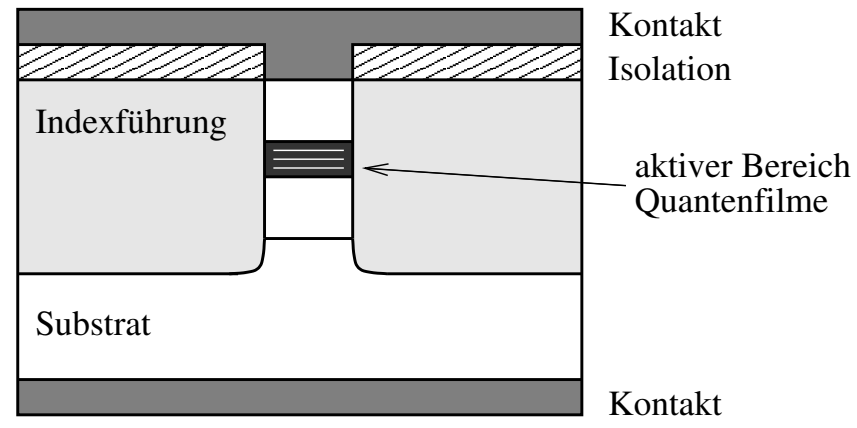

Abbildung 2.5: Aufbau eines kantenemittierenden Halbleiterlasers. Lichtaustritt in Richtung der Normalen.

Aufgrund der starken räumlichen Konzentration des Lichtes innerhalb des Halbleiterkristalls entsteht bei dem Austritt ein stark divergierender Strahl, der aufgrund der Form des Wellenleiters der EELs ein elliptisches Profil aufweist. Dies erschwert die Einkopplung in Glasfasern deutlich.

Die optische Polarisation ist hingegen bei diesem Lasertyp auf einfache Weise durch die Richtung der laseraktiven Schicht festgelegt. Sowohl die transversal elektrische TE-Mode als auch die transversal magnetische TM-Mode sind möglich. Aufgrund der geringeren Reflektivität der TM-Mode an den Kristallfacetten (siehe Abschnitt 2.5 in [3]) wird die Polarisationrichtung des Lasers im wesentlichen von der TE-Mode bestimmt, d.h. EELs sind im Allgemeinen linear polarisiert.

\subsection{Oberflächenemittierender Halbleiterlaser}

Im Gegensatz zum kantenemittierenden Laser liegt die Achse des Resonators eines oberflächenemittierenden Lasers (VCSEL - vertical cavity surface emitting laser) nicht in der Ebene der laseraktiven Schicht sondern senkrecht dazu (Abbildung 2.61). 
Bereits 1965 wurde von Melngailis [46] der erste oberflächenemittierende Halbleiterlaser gebaut, bei dem ein starkes Magnetfeld zur Ladungsträgerkonzentration eingesetzt werden musste. IGA verwendete 1977 erstmals Heterostrukturen in diesem Lasertyp. Diese ersten VCSELs besaßen eine enorm hohe Laserschwelle und mussten stark gekühlt werden $(900 \mathrm{~mA}$ bei $70 \mathrm{~K})$ 66. Grund war die ungenügende Reflektivität der aufgebrachten metallischen Verspiegelung. Durch den Einsatz von Halbleiterspiegeln konnten IBARAKI et al. 1984 [35] die Effizienz enorm steigern, Pulsbetrieb bei Raumtemperatur wurde möglich. Weitere Verbesserungen ergaben sich durch die Verwendung von Bragg-Reflektoren und Quantenfilmen in der laseraktiven Schicht. 1989 stellten JEWELL et al. einen oberflächenemittierenden Halbleiterlaser her, der bei Raumtemperatur im cw-Betrieb arbeiten konnte und eine Laserschwelle von nur $1 \mathrm{~mA}$ besaß [37]. Die ersten kommerziell erhältlichen VCSELs kamen 1996 auf den Markt.

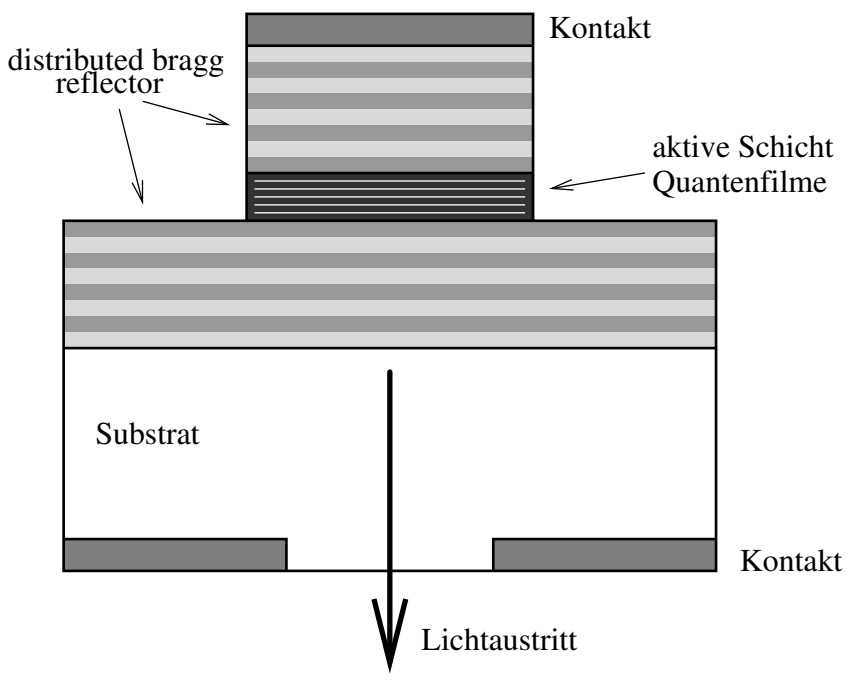

Abbildung 2.6: Aufbau eines oberflächenemittierenden Halbleiterlasers.

Da der Resonator mit Bragg-Reflektoren (DBR) ausgeführt wird, kann eine hohe Wellenlängenselektivität gewährleistet werden. VCSELs emittieren deshalb und wegen der im Vergleich zu EELs wesentlich kleineren Resonatorlänge im Allgemeinen nur eine longitudinale Mode pro Polarisationsrichtung.

Die Form des optischen Wellenleiters ist in der Ebene transversal zur Strahlrichtung nicht durch die Heterostruktur vorgegeben. Da das Strahlprofil des austretenden Laserstrahls entscheidend von dieser Form abhängt bietet sich eine kreisförmige Geometrie an, um eine einfache Einkopplung in eine Glasfaser zu gewährleisten. Durch diese Form ergeben sich jedoch einige Probleme. Die Polarisationsrichtung ist nicht wie bei den kantenemittierenden Halbleiterlasern auf einfache Weise festgelegt. Die 
Reflektivität der DBRs ist für beliebige Polarisationsrichtungen nahezu gleich. Durch leichte Ungenauigkeiten in der kreisförmigen Grundfläche, mechanischen Spannungen im Halbleiterkristall als auch durch die geringe Eigenschaft der Doppelbrechung des Kristalls kann eine Richtung ausgezeichnet werden, die jedoch von der Temperatur und noch weiteren Einflussfaktoren abhängen kann. Die Polarisation des Ausgangslichtes kann parallel als auch senkrecht zu dieser ausgezeichneten Richtung sein. In vielen VCSELs kann ein unmotiviertes Umschalten dieser Polarisationsmoden beobachtet werden [12. Durch die im Vergleich zu EELs große transversale Ausdehnung des Resonators treten in vielen VCSELs transversale Moden auf [11, 16, 17].

\subsection{Semiklassische Lasergleichungen}

In diesem Abschnitt soll die Herleitung der verwendeten semiklassischen Lasergleichungen skizziert werden. Es werden die wesentlichen Bestandteile speziell in Bezug auf den Halbleiterlaser vorgestellt sowie die üblichen Vereinfachungs- und Näherungsschritte dargestellt. Die Herleitung orientiert sich im Wesentlichen an Ref. [3] und [26].

Bei den semiklassischen Lasergleichungen werden die Gleichungen des elektromagnetischen Feldes mit den Maxwellgleichung - ohne Quantisierung des Feldes - beschrieben. Die Materiegleichungen werden jedoch quantenmechanisch betrachtet.

\section{Feldgleichungen}

Ausgangspunkt für die Feldgleichung des Halbleiterlasers sind die maxwellschen Gleichungen

$$
\begin{aligned}
\nabla \times \mathcal{E} & =-\frac{\partial \mathcal{B}}{\partial t} \\
\nabla \times \mathcal{H} & =\mathcal{J}+\frac{\partial \mathcal{D}}{\partial t} \\
\nabla \cdot \mathcal{D} & =\rho_{f} \\
\nabla \cdot \mathcal{B} & =0
\end{aligned}
$$

Für nichtmagnetische dielektrische Medien lassen sich folgende Beziehungen der Flussdichten zu den Feldern verwenden 


$$
\begin{aligned}
\mathcal{D} & =\epsilon_{0} \mathcal{E}+\mathcal{P} \\
\mathcal{B} & =\mu_{0} \mathcal{H} \\
\mathcal{J} & =\sigma \mathcal{E}
\end{aligned}
$$

mit $\epsilon_{0}$ als Permittivität und $\mu_{0}$ als Permeabilität des Vakuums sowie $\rho$ als Leitfähigkeit des Mediums.

Anwendung der Rotation auf Gleichung (2.2) ergibt mit Anwendung von (2.7)

$$
\nabla \times \nabla \times \mathcal{E}=-\mu_{0} \frac{\partial}{\partial t}(\nabla \times \mathcal{H})
$$

Durch Verwendung der Gleichungen (2.3), (2.6) und (2.8) lassen sich $\mathcal{H}, \mathcal{J}$ und $\mathcal{D}$ aus der Gleichung (2.9) eliminieren:

$$
\nabla \times \nabla \times \mathcal{E}=-\mu_{0} \sigma \frac{\partial \mathcal{E}}{\partial t}-\mu_{0} \epsilon_{0} \frac{\partial^{2} \mathcal{E}}{\partial t^{2}}-\mu_{0} \frac{\partial^{2} \mathcal{P}}{\partial t^{2}}
$$

Die linke Seite lässt sich durch die Identität

$$
\nabla \times \nabla \times \mathcal{E}=\nabla(\nabla \cdot \mathcal{E})-\nabla^{2} \mathcal{E}
$$

weiter vereinfachen. Ohne freie Ladungsträger gilt mit den Gleichungen (2.4) und (2.6)

$$
\nabla \cdot \mathcal{D}=\epsilon_{0} \nabla \cdot \mathcal{E}+\nabla \cdot \mathcal{P}=0
$$

Für den hier betrachteten Fall ist der Term $\nabla \cdot \mathcal{P}$ im Allgemeinen vernachlässigbar und es gilt $\nabla \cdot \mathcal{E}=0$. Gleichung (2.10) lässt sich dann wie folgt schreiben

$$
\nabla^{2} \mathcal{E}-\frac{\sigma}{\epsilon_{0} c^{2}} \frac{\partial \mathcal{E}}{\partial t}-\frac{1}{c^{2}} \frac{\partial^{2} \mathcal{E}}{\partial t^{2}}=\frac{1}{\epsilon_{0} c^{2}} \frac{\partial^{2} \mathcal{P}}{\partial t^{2}}
$$

unter Verwendung der Definition der Lichtgeschwindigkeit $\mu_{0} \epsilon_{0}=1 / c^{2}$.

Zur weiteren Vereinfachung werden die zeitliche und räumliche Änderung der Felder $\mathcal{E}$ und $\mathcal{P}$ separiert:

$$
\begin{aligned}
& \mathcal{E}(x, y, z, t)=\operatorname{Re}\left(\boldsymbol{E}(x, y, z) e^{i \omega t}\right) \\
& \mathcal{P}(x, y, z, t)=\operatorname{Re}\left(\boldsymbol{P}(x, y, z) e^{i \omega t}\right)
\end{aligned}
$$


Der Einfachheit halber wurde hierbei nur eine Frequenzkomponente mit der Kreisfrequenz $\omega$ betrachtet. Mit diesen beiden Gleichungen wird aus Gleichung (2.13)

$$
\nabla^{2} \boldsymbol{E}+k_{0}^{2}\left(1+\frac{i \sigma}{\epsilon_{0} \omega}\right) \boldsymbol{E}=-\frac{k_{0}^{2}}{\epsilon_{0}} \boldsymbol{P}
$$

Im stationären Zustand kann die Polarisation durch die elektrische Feldstärke ausgedrückt werden

$$
\boldsymbol{P}=\epsilon_{0} \chi(\omega) \boldsymbol{E},
$$

wobei die Suszeptibilität $\chi(\omega)$ im Allgemeinen komplex und frequenzabhängig ist. Gleichung (2.16) und Gleichung (2.17) ergeben die zeitunabhängige Wellengleichung

$$
\nabla^{2} \boldsymbol{E}+\epsilon k_{0}^{2} \boldsymbol{E}=0
$$

$\operatorname{mit} \epsilon=1+\chi(\omega)+i \sigma /\left(\epsilon_{o} \omega\right)$.

Für den Fall von zeitlich veränderlichen Feldern muss die Polarisation berücksichtigt werden. Da jedoch die Reaktionszeit vieler Medien sehr gering ist folgt die Polarisation bei den hier interessanten Zeitskalen den Änderungen des elektrischen Feldes ohne eine physikalisch relevante zeitliche Verzögerung. Aus diesem Grund wird die Polarisation bei der Beschreibung des Halbleiterlasers normalerweise adiabatisch eliminiert. Anders ausgedrückt bedeutet dies, dass die Gleichung (2.17) auch für zeitlich langsam veränderliche Felder $\mathcal{E}$ und $\mathcal{P}$ als gültig angenommen wird. Diese seien nun unter Vernachlässigung des Realteils in den Gleichungen (2.14) und (2.15) komplexwertig. Die Feldgleichung (2.18) sieht dann wie folgt aus:

$$
\nabla^{2} \mathcal{E}-\frac{1}{c^{2}} \frac{\partial^{2}}{\partial t^{2}}(\epsilon \mathcal{E})=0
$$

Die räumliche Modenstruktur lässt sich als Superposition von ebenen Wellen auffassen

$$
\mathcal{E}(\boldsymbol{x}, t)=\mathcal{E}(x, y, z, t)=\psi(x) \phi(y) \sum_{\lambda} E_{\lambda}(t) \boldsymbol{u}_{\lambda}(\boldsymbol{x})
$$

mit

$$
\boldsymbol{u}_{\lambda}(\boldsymbol{x})=\boldsymbol{e}_{\lambda} \mathcal{N} \sin k_{\lambda} z .
$$

Hierbei ist $\boldsymbol{e}_{\lambda}$ der Einheitsvektor in Polarisationsrichtung und $\mathcal{N}$ eine geeignete Normierungskonstante. Es wurde hierbei auch nur der multilongitudinale, monotransversale Fall betrachtet. $k_{\lambda}$ ist die Wellenzahl der longitudinalen $z$-Richtung.

Die zweite räumliche Ableitung in (2.18), angewendet auf $\boldsymbol{u}_{\lambda}$ ergibt

$$
\nabla^{2} \boldsymbol{u}_{\lambda}(\boldsymbol{x})=-k_{\lambda}^{2} \boldsymbol{u}_{\lambda}(\boldsymbol{x})=-\frac{\omega_{\lambda}^{2}}{c^{2}} \boldsymbol{u}_{\lambda}(\boldsymbol{x})
$$


Durch Einsetzen von $\mathcal{E}$ aus Gleichung (2.20) in Gleichung (2.19) ergibt nach Integration über die transversalen Richtungen $x$ und $y$

$$
\frac{2 i \omega}{c^{2}}\left(\langle\epsilon\rangle+\frac{\omega}{2} \frac{\partial\langle\epsilon\rangle}{\partial \omega}\right) \frac{\mathrm{d}}{\mathrm{d} t} E(t)+\left(\frac{\omega^{2}}{c^{2}}\langle\epsilon\rangle-k^{2}\right) E(t)=0
$$

Hierbei wurden einige Vereinfachungen verwendet und angenommen $E(t)$ ändere sich nur langsam. Für räumlich gemittelte Dielektrizitätskonstante gilt:

$$
\langle\epsilon\rangle=\int_{-\infty}^{\infty} \int_{-\infty}^{\infty} \epsilon(x, y) \psi^{2}(x) \phi^{2}(y) d x d y \approx \bar{\mu}^{2}
$$

Hier wurde näherungsweise angenommen, dass $\langle\epsilon\rangle$ konstant ist mit $\bar{\mu}$ als gemittelten Brechungsindex des Mediums. Weiter gilt für $k=\bar{\mu} \omega_{0} / c$. Mit der Vereinfachung $\left(\omega^{2}-\right.$ $\left.\omega_{0}^{2}\right) \approx 2 \omega\left(\omega-\omega_{0}\right)$ lässt sich Gleichung (2.23) weiter vereinfachen:

$$
\frac{\mathrm{d}}{\mathrm{d} t} E(t)=\left(i\left(\omega-\omega_{0}\right)+\frac{1}{2}\left(G-\frac{1}{\tau_{p}}\right)\right) E(t)
$$

Der Brechungsindex $\mu$ des Mediums und demnach auch die Frequenz $\omega$ hängen noch von der Ladungsträgerinversion $N(t)$, also von später beschriebene Materiegleichung ab. In diesem Zusammenhang wird häufig der Linienbreitenvergrößerungsfaktor (engl. linewidth enhancement factor) oder auch $\alpha$-Faktor verwendet:

$$
\alpha=-\frac{\operatorname{Re} \chi_{p}}{\operatorname{Im} \chi_{p}}=-k_{0} \frac{\partial \mu / \partial N}{\partial G / \partial N}
$$

Die Feldgleichung (2.25) lässt sich unter Zuhilfenahme dieses $\alpha$-Faktors mit der Beziehung

$$
\omega=\omega_{0}+\frac{1}{2} \alpha G(N)
$$

weiter vereinfachen.

\section{Materiegleichung}

Bei den herkömmlichen semiklassischen Lasergleichungen wird die Materiegleichungen durch die quantenmechanische Berechnung des Polarisation hergeleitet. Da jedoch für die hier betrachteten langsameren physikalischen Vorgänge die Polarisation adiabatisch eliminiert werden kann, bietet sich ein phänomenologischer Ansatz an. Sowohl numerisch als auch experimentell lässt sich zeigen, dass die Gewinnfunktion $G(N)$ für 
jeden beliebigen Pumpstrom $J$ im wesentlichen linear mit der Ladungsträgerinversion steigt. D.h.

$$
G(N)=G_{N}\left(N-N_{t r}\right)
$$

wobei $G_{N}$ der differentielle Gewinn und $N_{t r}$ die Ladungsträgerinversion bei Transparenz ist.

Desweiteren muss ein Quellterm in Form des Pumpstromes $J$ und ein Dissipationsterm für Ladungsträger in der Materiegleichung Verwendung finden. Ist der Laser gewinngeführt so darf auch die Ladungsträgerdiffusion nicht vernachlässigt werden:

$$
\frac{\mathrm{d}}{\mathrm{d} t} N(t)=D \nabla^{2} N(t)+J-\gamma N(t)-G(N)|E(t)|^{2}
$$

Hierbei ist $D$ der Diffusionskoeffizient und $\gamma$ der Koeffizient für die Ladungsträgerverluste, die keine Strahlung produzieren. Der Diffusionskoeffizient kann jedoch im weiteren vernachlässigt werden, da er für die verwendeten indexgeführten Laserdioden nur eine untergeordnete Rolle spielt.

Die kompletten Lasergleichungen sind dann:

$$
\begin{aligned}
\frac{\mathrm{d}}{\mathrm{d} t} E(t) & =\left[\frac{i \alpha}{2} G(N)+\frac{1}{2}\left(G(N)-\frac{1}{\tau_{p}}\right)\right] E(t) \\
\frac{\mathrm{d}}{\mathrm{d} t} N(t) & =J-\gamma N(t)-G(N)|E(t)|^{2}
\end{aligned}
$$

\subsection{Multi-Longitudinale Lasergleichungen}

Gleichungen für einen Halbleiterlaser mit mehreren longitudinalen Moden lassen sich ohne großen Aufwand aus diesen Monomodegleichungen gewinnen. Dafür ist lediglich die Definition der spektralen Gewinnverteilung der Moden nötig. Die einfachste Näherung geht hierbei von einem quadratischen Abfall des Gewinns zu niedrigeren und höheren Frequenzen ausgehend von einem Maximum bei $\omega_{0}$ aus.

$$
G(N, \omega)=G_{N}\left(N-N_{t r}\right)\left[1-\left(\frac{\omega-\omega_{0}}{\Delta \omega_{g}}\right)^{2}\right]
$$

wobei $\Delta \omega_{g}$ die spektrale Breite zwischen der untersten und der obersten anschwingenden Mode beschreibt. Die Modenfrequenzen sind hierbei

$$
\omega_{m}=\omega_{0}+m \Delta \omega_{L}
$$


mit $\Delta \omega_{L}$ als spektralem Modenabstand und $-M \leq m \leq+M, m \in \mathbb{Z}$ und $M \approx$ $\Delta \omega_{g} / \Delta \omega_{L}, M \in \mathbb{N}$

Die gesamten Multimode-Lasergleichungen sind dann:

$$
\begin{aligned}
\frac{\mathrm{d}}{\mathrm{d} t} E_{(m)}(t) & =\left[\frac{i \alpha}{2} G\left(N, \omega_{m}\right)+\frac{1}{2}\left(G\left(N, \omega_{m}\right)-\frac{1}{\tau_{p}}\right)\right] E_{(m)}(t) \\
\frac{\mathrm{d}}{\mathrm{d} t} N(t) & =J-\gamma N(t)-\sum_{m} G\left(N, \omega_{m}\right)\left|E_{(m)}(t)\right|^{2}
\end{aligned}
$$

Hierbei wird für jede anschwingende Mode $m$ eine eigene Feldgleichung aufgestellt. Normalerweise kann davon ausgegangen werden, dass die optischen Verluste $1 / \tau_{p}$ für alle Moden gleich und dementsprechend wellenlängenunabhängig sind.

\subsection{Modensprünge}

Eine wesentliche Eigenschaft des Halbleiterlasers ist die einfache Beeinflussung der emittierten Lichtfrequenz. Sowohl durch Temperatur- als auch durch Pumpstromvariation lässt sich die Lichtfrequenz verändern. Die Temperatur verändert u.A. die mechanische Länge des internen Resonators, wodurch sich die Resonanzbedingung und damit der Lichtfrequenz minimal verändert. Über den Pumpstrom lässt sich die Ladungsträgerdichte in der aktiven Schicht einstellen; der Brechungsindex des Halbleitermaterials besitzt eine leichte Abhängigkeit von dieser Dichte wodurch sich die optische Länge des internen Resonators ebenfalls geringfügig ändert.

Die Gewinnkurve des Halbleitermaterials besitzt aufgrund der Temperaturabhängigkeit der Bandlücke [85] eine deutlich stärkere Abhängigkeit von der Temperatur als der Resonator selber. Aus diesem Grund kommt es zu dem Phänomen der Modensprünge (engl. mode hopping): Durch die starke Verschiebung der Gewinnkurve fallen bisher nicht verstärkte longitudinale Resonatormoden in den Bereich der Gewinnkurve und bisher verstärkte Moden fallen aus der Gewinnkurve. Dies tritt lediglich an diskreten Punkten des variierten Parameters auf, an denen sich die Frequenzen der beteiligten Moden im Allgemeinen sprunghaft ändern. Dies wird als deterministischer Modensprung bezeichnet.

In der Nähe dieser diskreten Punkte kann ein spontanes Hin- und Herspringen der neuen und alten Modenfrequenzen beobachten werden. Dies wird im wesentlichen durch den Rauscheinfluss auf die Laserparameter hervorgerufen. Ich verwende zur Unterscheidung von den oben beschriebenen deterministisch begründeten Modensprüngen hierfür den Begriff der spontanen Modensprünge, da sie für bestimmte Parameterkombinationen ohne äußere Beeinflussung des Lasers auftreten. 



\section{Kapitel 3}

\section{Halbleiterlaser mit optischer Rückkopplung}

Zur Herstellung von Einmodenlasern wurde außer den besprochenen DFB- und DBRLasern auch Laser mit mehr als einem Resonator verwendet. Nachteil der DFB- und DBR-Laser ist die durch die feste, alternierende Abfolge verschiedener Brechungsindizes festgelegte Wellenlänge des Lasers. Die Lichtfrequenzen dieser Laser sind demnach nicht abstimmbar obwohl bei herkömmlichen kantenemittierenden Lasern dies problemlos über den Pumpstrom und die Temperatur realisiert werden kann.

Für spezielle Anwendungen wurden deshalb Halbleiterlaser entwickelt, die durch mehrere Resonatoren einen Einzelmodenbetrieb auf abstimmbarer Lichtfrequenz ermöglichten, wobei die Idee dieser gekoppelten Resonatoren (engl. coupled cavities) bereits bei Gaslasern Verwendung fand. Die vielen verwendeten Konzepte unterscheiden sich durch die Anordnung und die Art der verwendeten Resonatoren.

Ich beschränke mich im folgenden auf die Beschreibung der in dieser Arbeit verwendeten Halbleiterlaser mit passivem, externem Resonator (den ECSL - external cavity semiconductor laser). Eine umfangreiche Darstellung anderer Typen dieser Laser ist in Ref. [3] zu finden.

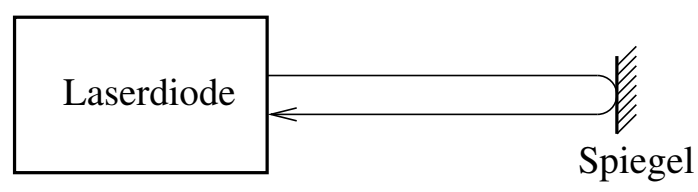

Abbildung 3.1: Laser mit externem Resonator

Bei einem Halbleiterlaser mit externem Resonator wird durch einen entfernt stehenden Spiegel ein Teil des emittierten Lichtes in den Laser zurückreflektiert. Durch die geringe Spiegelqualität der Laserfacetten des Lasers und aufgrund des vergleichsweise großen $\alpha$-Faktors ist eine starke Beeinflussung des Lasers zu erwarten. 
Eine der ersten experimentellen und numerischen Untersuchung zu diesen optisch rückgekoppelten Lasern wurde 1980 von LANG und KobaYASHI veröffentlicht [41. Die beobachteten Phänomene hängen hierbei sehr stark von der Länge des Resonators, der Menge und Phase des reflektierten Lichtes als auch von dem Pumpstrom des Lasers und dessen Temperatur ab. LANG und KoBAYASHI konnten bei den von ihnen verwendeten externen Resonatorlängen von $1-2 \mathrm{~cm}$ und geringer Spiegelreflektivität Multistabilität sowie Hysterese-Phänomene, ähnlich einem nichtlinearen Fabry-PerotResonator, beobachten und numerisch simulieren. Sie stellten in dieser Arbeit das in Abschnitt 3.2 beschriebene Gleichungssystem zur numerischen Simulation auf.

Andere Arbeitsgruppen konnten später bei genau abgestimmter Phasenbeziehung des externen Resonators eine deutliche Reduktion der Linienbreite feststellen [2, 52, 71]. Im Gegensatz dazu kann jedoch eine ungünstige Phasenbeziehung zu eine deutliche Linienverbreiterung auf über $25 \mathrm{GHz}$ führen. Dieses Phänomen wird als Kohärenzkollaps bezeichnet, da dabei die Kohärenz des Lichtes zerstört wird [42].

\subsection{Low Frequency Fluctuations}

Ein sehr auffälliges Phänomen für den Fall von Resonatorlängen im Bereich von 10$100 \mathrm{~cm}$ sowie geringer Reflektivität des Rückkoppelspiegels sind die low frequency fluctuations (LFFs). Sie wurden erstmals von RISCH und Voumand 1977 in [56] beschrieben. Die Intensität des Lasers zeigt hierbei mit einer Frequenz von nur 3-30MHz sehr abrupte Intensitätseinbrüche (Abb. 3.2) mit nachfolgender langsamerer Erholung auf den alten Wert. Aufmoduliert auf diese langsame Struktur scheint ein äußerst hochfrequentes Signal zu liegen, welches im Experiment, je nach Bandbreite der verwendeten Fotodetektoren, mehr oder weniger stark herausgefiltert wird. Diese Dynamik entsteht bei Pumpströmen knapp oberhalb der Laserschwelle und geringen bis mittleren Spiegelreflektivitäten.

Verschiedene Erklärungsmodelle für diese Strukturbildung wurden in den folgenden zwei Jahrzehnten publiziert. Hierbei gab es zwei grundsätzlich unterschiedliche Interpretationen des Phänomens. Auf der einen Seite wurden rauschinduzierte Modelle vorgeschlagen. HenrY und KaZARINOv gingen davon aus, dass der Laser durch das Rauschen seine normalerweise stabilen Resonatormode verlassen kann. Dieses führt dann zu einem Intensitätseinbruch [30]. MøRK et al. nahmen an, dass der Laser im Falle der Rückkopplung bistabil wird und auf zwei unterschiedlich starken Moden arbeiten kann [4]. Durch das natürliche Rauschen in Form der spontanen Emission findet ein Hin- und Herspringen zwischen beiden Zuständen statt. 


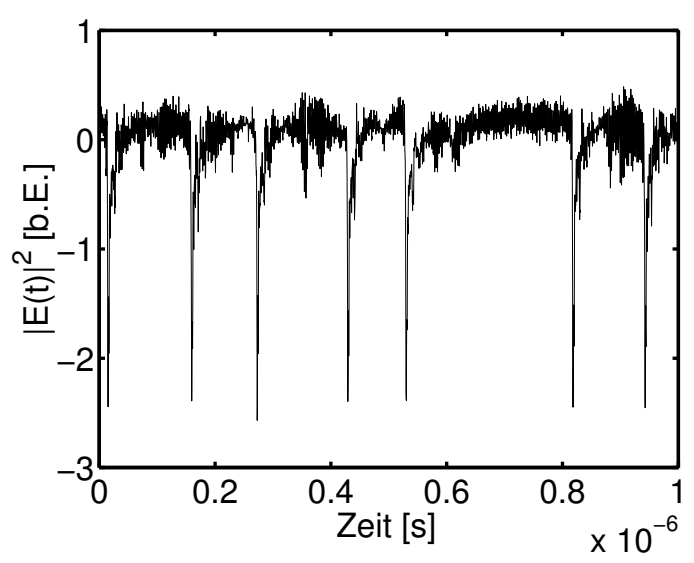

Abbildung 3.2: LFF-Dynamik einer Laserdiode.

Auf der anderen Seite gab es die Vertreter der deterministischen Modelle. SAno zeigte 1994, dass die LFFs direkt mit den bekannten Lang-Kobayashi-Modellgleichungen simulierbar sind 61]. Durch Stabilitätsanalyse dieses Systems konnte er darüberhinaus eine elegante Erklärung dieses Phänomens liefern, die ich in Abschnitt 3.3 skizzieren werde.

Die numerische Simulation mit den Lang-Kobayashi Gleichungen zeigte die interessante Struktur der LFFs in hoher zeitlicher Auflösung. Das Intensitätssignal beschreibt hierbei sehr schnelle, intensive Pulse (Abb. 3.3) . Nur durch die im Experiment unvermeidbare Tiefpassfilterung zeigen sich die bekannten langsamen Fluktuationen.

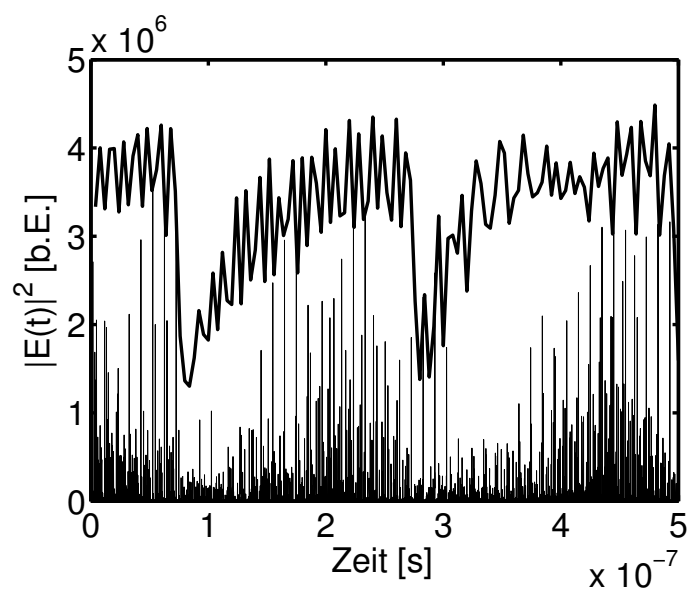

Abbildung 3.3: Intensitätssignal der numerischen LFF-Simulation. Dünne Linie: ungefilterte Intensität, dicke Linie: tiefpassgefiltert und hochskalierte Intensität.

Diese durch VAN TARTWIJK et al. 1995 [77] numerisch vorhergesagten Pulse konnten durch Fischer et al. 20] ein Jahr später experimentell nachgewiesen werden. Diese 
Arbeiten konnten auch zeigen, dass der Kohärenzkollaps die selbe schnelle Pulsdynamik aufweist wie die LFFs, jedoch ohne die dort sichtbaren Intensitätseinbrüche der Hüllkurve. HEIL et al. veröffentlichten 1998 in einer systematischen experimentellen Studie [29] für verschiedene Pumpströme und Spiegelreflektivitäten einen Überblick der möglichen Dynamiken.

Nicht enthalten in diesem Überblick ist die Entdeckung der Intensitätssprünge (engl. power jump-ups) durch DE TOMASI et al. [15 1994. Diese Dynamik zeigt ebenfalls langsame Fluktuationen, jedoch im Gegensatz zu den bekannten LFFs bricht hierbei die Intensität nicht ein sondern springt auf ein höheres Niveau (Abb. 3.4). Deutlich höhere Pumpströme und Reflektivitäten des Spiegels sind im Experiment für diese Dynamik nötig. DE TOMAsI et al. fanden jedoch heraus, dass sich diese Dynamik nur auf der Seite des externen Resonators als Intensitätsprünge manifestiert. An der anderen Laserfacette, die nicht dem externen Resonator zugewandt ist, sind die bekannten Intensitätseinbrüche beobachtbar. Es besteht also eine Asymmetrie in der Lichtemission zwischen den beiden Laserfacetten. SPENCER et al. veröffentlichten 1997 67] ein iteratives Modell, welches die beschriebenen Intensitätssprünge in Verbindung mit den bei der LFF-Dynamik gefundenen schnellen Pulsfolgen zeigt. Eine experimentelle Bestätigung steht hierzu noch aus.

Da es sich bei beiden Dynamiken sinngemäß um langsame Fluktuationen, also low frequency fluctuations handelt, gehe kurz auf die in dieser Arbeit verwendeten Begriffe ein: Für die Dynamik mit den Intensitätseinbrüchen wurde von PAN et al. der Begriff power drop-outs oder genauer low frequency power drop-outs verwendet. Ich verwende hierfür neben der Bezeichnung Intensitätseinbrüche auch die Abkürzung LFF. Diese Abkürzung wird in allen mir bekannten Veröffentlichungen in dieser Weise verwendet. Die Dynamik mit den Intensitätssprüngen wurde sinngemäß power jump-ups oder low frequency power jump-ups genannt. Dementsprechend verwende ich in dieser Arbeit neben dem Begriff Intensitätssprünge auch die Abkürzung LFJ (für Low Frequency power Jump-ups).

\subsection{Die Lang-Kobayashi-Modellgleichungen}

Für beliebige Reflektivitäten des externen Spiegels kann nicht mehr angenommen werden, dass sich im Laserresonator eine stehende Welle aufbaut. Für geringe bis mittlere Reflektivitäten hat sich jedoch gezeigt, dass die bereits hergeleitete Feldgleichung (2.30) weiterverwendet werden kann. In diesem Fall kann der Einfluss des zurückreflektierten Lichtes als kleine Störung angesehen werden, die die räumliche Modenstruktur 


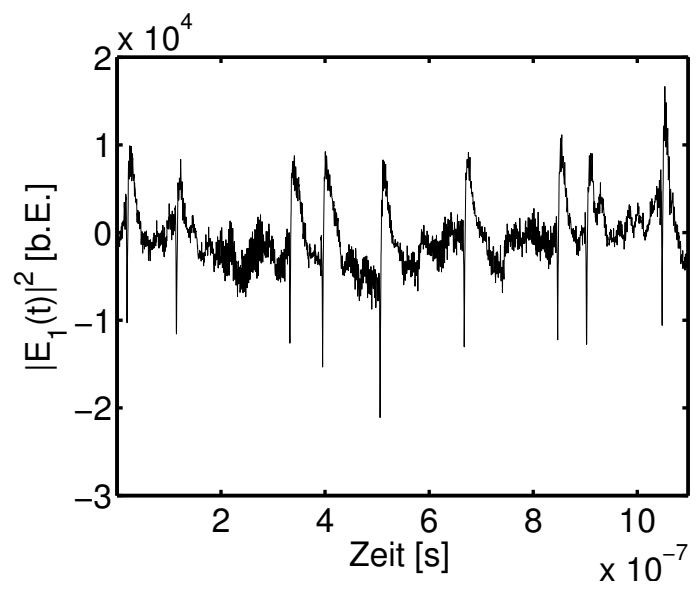

Abbildung 3.4: Dynamik der Intensitätssprünge einer optisch rückgekoppelten Laserdiode.

innerhalb des Lasers nur unwesentlich beeinflusst. Von Interesse sind dann vor allem die zeitlichen Einflüsse dieser Rückkopplung, die durch einen zusätzlichen Term Eingang in die Feldgleichung findet

$$
\frac{\mathrm{d}}{\mathrm{d} t} E(t)=\left[\frac{i \alpha}{2} G(N)+\frac{1}{2}\left(G(N)-\frac{1}{\tau_{p}}\right)\right] E(t)+\kappa E(t-\tau) e^{-i \omega_{0} \tau},
$$

wobei $\tau$ die Umlaufzeit im externen Resonator beschreibt. Hierbei wird auch nur ein einziger Umlauf im externen Resonator berücksichtigt, da angenommen wird, dass alle weiteren Umläufe aufgrund der geringen Intensität keinen Einfluss mehr haben. Die Materiegleichung ist identisch mit der der einfachen Laserdiode (2.31).

Entwickelt wurden diese Gleichung für die Simulation von rein kohärenter Rückkopplung. Ist die Resonatorlänge wesentlich größer als die Kohärenzlänge des Lasers muss von inkohärenter Kopplung ausgegangen werden. Die von mir verwendeten Laserdioden besitzen viele longitudinale Moden und demnach eine Kohärenzlänge von maximal einigen Millimetern. Bei einer Resonatorlänge von ca. $35 \mathrm{~cm}$ ist von inkohärenter Kopplung auszugehen. In den Achtziger und Neunziger Jahren des vergangenen Jahrhundert wurden die Kohärenzeigenschaften des optisch rückgekoppelten Halbleiterlasers im Bereich des Kohärenzkollapses intensiv untersucht [13, 14, 28, 42. Zur Modellierung dieser Dynamik zeigte sich, dass trotz der offensichtlich inkohärenten Kopplung die Einbeziehung der Phase zwingend erforderlich war. Zwar existiert keine feste Phasenbeziehung mehr zwischen dem Feld des Lasers $E(t)$ und dem rückgekoppelten Feld $\kappa E(t-\tau)$, doch wurde in den Veröffentlichungen angenommen, dass die demnach zufällig fluktuierende Phasendifferenz als eine Art Rauschterm wirkt. Dies ist auch eine Erklärung dafür, dass die numerische Modellierung der spontanen Emission 
einen fast verschwindenden Einfluss auf die Dynamik des Systems hat. Der Einfluss des Phasenrauschens ist weit größer. In vielen der folgenden Veröffentlichungen und auch in dieser Arbeit, wurde deshalb die spontane Emission vernachlässigt.

Es sei hier noch erwähnt, dass in einigen Veröffentlichungen die im LK-Modell verwendete Kopplung im Sinne von LANG und KOBAYASHI grundsätzlich als „kohärent“ bezeichnet wird [61].

Die Rückkoppelrate $\kappa$ ist hierbei definiert als

$$
\kappa=\frac{1-r_{2}^{2}}{\tau_{\text {in }}} \cdot \frac{r_{3}}{r_{2}}
$$

Hierbei beschreibt $r_{2}$ die Amplitudenreflektivität der dem externen Resonator zugewandten Laserfacette und $r_{3}$ die des Spiegels. $\tau_{i n}$ bezeichnet die Umlaufzeit im internen Resonator der Laserdiode.

\subsection{Fixpunkte und Stabilität}

Zum Auffinden und der Analyse der Fixpunkte ist eine Spaltung der Feldgleichung in Betrag und Phase sinnvoll:

$$
E(t)=E_{0}(t) e^{i\left(\omega_{0} t+\phi(t)\right)}
$$

Aus Gleichung (3.1) wird dann

$$
\begin{aligned}
\frac{\mathrm{d}}{\mathrm{d} t} E_{0}(t) & =\frac{1}{2} G_{N} n(t) E_{0}(t)+\kappa E_{0}(t-\tau) \cos \left(\omega_{0} \tau+\phi(t)+\phi(t-\tau)\right) \\
\frac{\mathrm{d}}{\mathrm{d} t} \phi(t) & =\frac{1}{2} G_{N} \alpha n(t)-\kappa \frac{E_{0}(t-\tau)}{E_{0}(t)} \sin \left(\omega_{0} \tau+\phi(t)-\phi(t-\tau)\right)
\end{aligned}
$$

Hierbei wurde noch $n(t)=N(t)-N_{t h}$ verwendet mit $N_{t h}$ als Ladungsträgerinversion des Lasers an der Laserschwelle.

Für stationäre Lösungen gilt

$$
E_{0}(t)=\tilde{E}_{0}, \quad \phi(t)=\tilde{\phi} t, \quad N(t)=\tilde{n}
$$

mit konstanten $\tilde{E}_{0}, \tilde{\phi}, \tilde{n}$. (3.6) eingesetzt in (3.4) und (3.5) ergibt

$$
\begin{aligned}
G_{N} \tilde{n} & =-2 \kappa \cos (\omega \tau) \\
\tilde{E}_{0}^{2} & =\frac{E_{S}^{2}-\tau_{p} \gamma \tilde{n}}{1+G_{N} \tau_{p} \tilde{n}} \\
\tilde{\phi} & =-\kappa(\alpha \cos \omega \tau+\sin \omega \tau) \\
& =-\kappa \sqrt{1+\alpha^{2}} \sin \left(\omega_{0} \tau+\tilde{\phi}+\arctan \alpha\right)
\end{aligned}
$$


mit $\omega=\omega_{0}+\tilde{\phi}$ und $E_{S}$ als Feldamplitude des konstant leuchtenden Lasers. Die transzendente Gleichung (3.9) lässt sich zusammen mit der Gleichung (3.7) auf anschauliche Weise grafisch lösen. Die Lösungen dieser Gleichungen sind die Schnittpunkte der beiden Kurven in Abbildung 3.5. Die rote Kurve beschreibt die Ladungsträgerbedingung aus Gleichung (3.7), die blaue Kurve die Phasenbedingung aus Gleichung (3.9) 1 . In dieser Darstellung liegen die Fixpunkte auf dem Rand einer Ellipse [61], in der Abbildung [3.5 grün dargestellt.

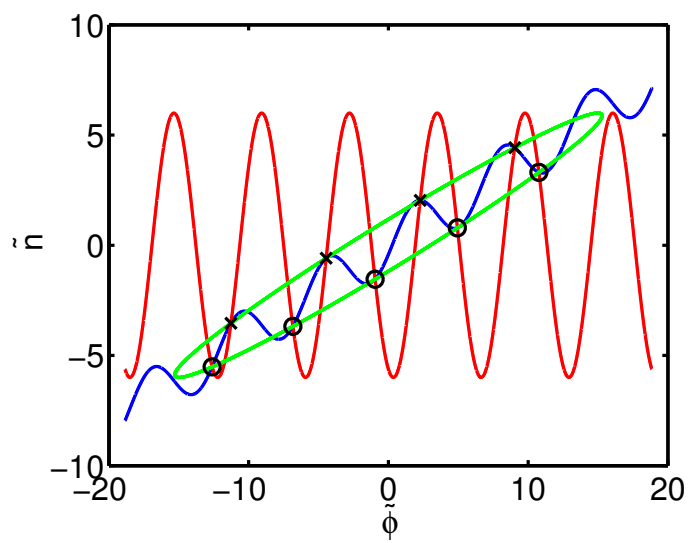
Abbildung 3.5: Fixpunkte der Lasergleichung, aufgetragen in einem $\tilde{\phi}-\tilde{n}$ - Diagramm. Rot: Ladungsträgerbedingung aus Gleichung (3.7), blau: Phasen- bedingungen aus Gleichung (3.9), grün: Lösungsraum, $\times$ Antimoden, ○ Eigen- moden.

Eine detaillierte Analyse dieser Fixpunkte haben Mørk et al. 1992 in [49] ausgeführt. Sie zeigten, dass alle Fixpunkte in der oberen Hälfte der Ellipse instabile Sattelknoten darstellen. Diese auch als Antimoden bezeichneten Fixpunkte repräsentieren eine destruktive Interferenz des Laserfeldes mit dem rückgekoppelten Feld. Die Fixpunkte der unteren Ellipsenhälfte sind je nach den eingestellten Parametern stabil oder durch mehrere Hopfbifurkation zu instabilen Grenzzyklen geworden. Diese Fixpunkte werden Eigenmoden oder externe Resonatormoden (engl. ECMs - external cavity modes) genannt. Sie sind auf eine konstruktive Interferenz des Laserfeldes mit dem rückgekoppelten Feld zurückzuführen.

Der Mechanismus, der den LFFs zugrunde liegt wurde von SANO [61] anschaulich wie folgt beschrieben. Ausgangspunkt der Dynamik ist die Mode des Lasers ohne Einfluss des externen Spiegels in der Mitte der Ellipse. Durch den Einfluss des externen Resonators ist diese Mode instabil und es werden benachbarte ECMs aufgesucht. Durch die dort ausgeführte chaotische Dynamik werden durch die nichtverschwindende Bandbreite des Signals Frequenzen benachbarter ECMs angeregt und nehmen an der

\footnotetext{
${ }^{1}$ Hierbei wurde Gleichung (3.7) eingesetzt und nach $\tilde{n}$ aufgelöst.
} 
Kap. 3 Halbleiterlaser mit optischer Rückkopplung

chaotischen Dynamik teil. Bogatov et al. [7] konnten 1975 zeigen, dass die Energie der Moden in einem Halbleiterlaser in Richtung der kleineren Frequenzen transportiert wird. Die Dynamik des Lasers wandert also chaotisch entlang des unteren Teiles der Ellipse von Eigenmode zu Eigenmode (Abb. 3.6). Die Intensität des Laser schaukelt sich hierbei langsam auf, da die Ladungsträgerinversion $\tilde{n}$ abnimmt. In den meisten Fällen trifft die Trajektorie zu einem späteren Zeitpunkt in dieser Aufschaukelphase auf eine Antimode. Durch diese destruktive Interferenz des Laserfeldes mit dem externen Feld, wird die Intensität des Lasers praktisch auf Null reduziert. Im Phasenraum in Abbildung 3.6 bedeutet dies ein sofortiger Anstieg der Ladungsträgerinversion auf das Niveau der Laserschwelle. Der Laser schwingt wieder in der stationären Mode ohne externen Resonator an und das Spiel beginnt von neuem.

LEVINE et al. konnte zeigen, dass die Eigenmode mit der kleinsten Ladungsträgerinversion $n(t)$ (und demnach maximalen Intensität, maximum gain mode, MGM) stabil sein muss [43. Dies gilt unter bestimmten Umständen auch für in der Nähe liegende Eigenmoden.

Erreicht die Trajektorie also bei dem Durchwandern des Bereiches des Eigenmoden die MGM, so ist es möglich, dass der Laser keine weiteren Intensitätseinbrüche zeigt und mit maximaler Intensität konstant emittiert. Die Möglichkeit des Eintretens dieses Ereignisses hängt jedoch sehr stark von den Parametern, u. a. von der Reflektivität des externen Resonators ab. Für geringe Reflektivitäten ist das Erreichen der MGM nicht möglich, wodurch VAN TARTWIJK et al. 1995 in [77] für dieses Problem auch den Begriff des Sisyphus-Effekts prägten. FiscHeR et al. konnten 1998 [19] numerisch und experimentell zeigen, dass unter bestimmten Bedingungen das Erreichen der MGM möglich ist und die LFF-Dynamik in diesem Fall lediglich ein transientes Phänomen darstellt.

\subsection{Multimode Lang-Kobayashi-Modellgleichungen}

Die Modellgleichungen von LANG und KoBAYASHI gehen nur von einer einzigen longitudinalen Mode aus. Genaugenommen beschreiben diese Modellgleichungen also nur für DFB/DBR-Laser oder Laserdioden mit frequenzselektiver Rückkopplung den physikalischen Aufbau. Der in Abbildung 3.1] dargestellte Aufbau zeigt bei Verwendung von kantenemittierenden Lasern im Allgemeinen eine multilongitudinale Dynamik, wie an dem Spektrum in Abbildung 3.7 zu erkennen ist.

Das lange Festhalten an dem normalen Lang-Kobayashi-Modell auch für multimodale Laser wurde häufig damit begründet, dass in einem solchen Laser wahrscheinlich eine 


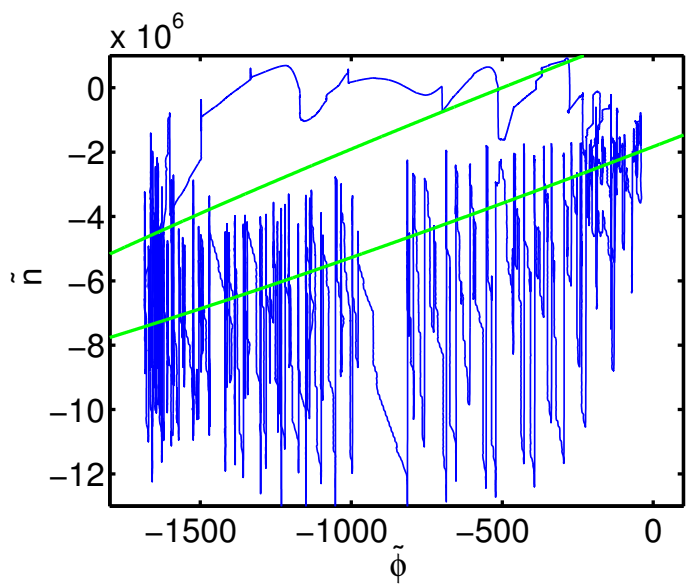

Abbildung 3.6: Phasenportrait eines Intensitätsaufbaus und Einbruchs bei LFF-Dynamik in der $\tilde{\phi}-\tilde{n}$-Ebene. Die Fixpunkte liegen sehr dicht entlang der grünen Ellipse (nur ein Ausschnitt erkennbar).

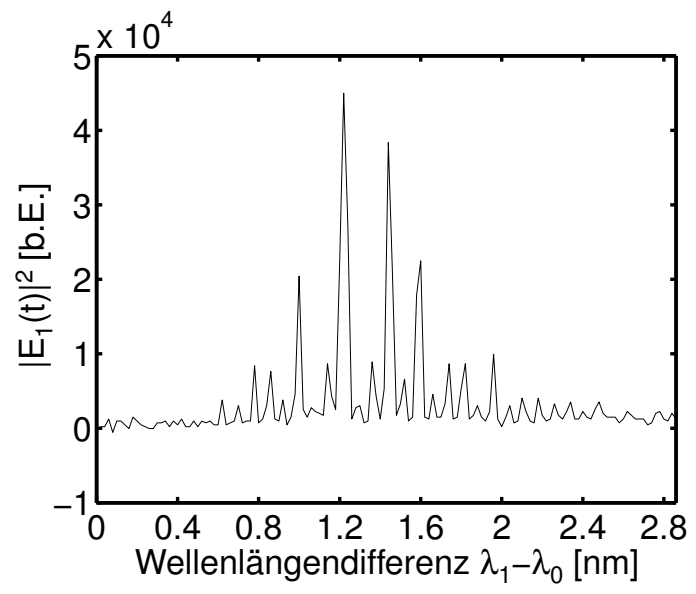

Abbildung 3.7: Experimentell aufgenommenes Spektrum eines optisch rückgekoppelten, kantenemittierenden Diodenlasers bei LFF-Dynamik. 
Mode eine Führungsrolle übernimmt und alle weiteren Moden synchron mitschwingen. Zur Modellierung wäre es dann ausreichend, nur diese Führungsmode zu beschreiben.

Huyet et al. fanden 1997 [33] erste Indizien gegen diese Annahme und konnte bei einer genaueren Untersuchung des zeitaufgelösten Spektrums 1998 in [32] keine solche Führungsmode ausfindig machen. Dies konnte durch VASCHENKO et al. im selben Jahr mit einem anderen Messaufbau bestätigt werden [78. Die wesentlichen Erkenntnisse dieser Arbeiten war die nicht synchrone Phase der einzelnen longitudinalen Moden.

Sukow et al. verwendeten in ihrer Veröffentlichung 1999 [69] eine Erweiterung des LKModells auf mehrere Moden. Unter Vernachlässigung der Feldsättigung verwendeten Rogister et al. [57] ebenfalls dieses Modell:

$$
\begin{aligned}
\frac{\mathrm{d}}{\mathrm{d} t} E_{(m)}(t)= & {\left[\frac{i \alpha}{2} G\left(N, \omega_{m}\right)+\frac{1}{2}\left(G\left(N, \omega_{m}\right)-\frac{1}{\tau_{p}}\right)\right] E_{(m)}(t) } \\
& +\kappa_{m} E_{(m)}(t-\tau) e^{-i \omega_{m} \tau} \\
\frac{\mathrm{d}}{\mathrm{d} t} N(t)= & p J_{t h}-\gamma N(t)-\sum_{m} G\left(N, \omega_{m}\right)\left|E_{(m)}(t)\right|^{2}
\end{aligned}
$$

Dieses Modell entspricht dem bereits eingeführten multimodalen Lasermodell ohne optischer Rückkopplung aus Abschnitt 2.5. Für die Rückkopplung wird angenommen, dass jede einzelne Mode lediglich mit sich selbst koppelt. VikTorov et al. [79] verwendeten ein etwas aufwendigeres Modell, indem sie auch für jede Mode eine eigene Ladungsträgerinversion $N_{(m)}$ verwendeten. Rechtfertigen lässt sich dies durch den Einfluss des s.g. carrier gratings: Unterschiedliche Moden räumen die Ladungsträgerinversion an räumlich unterschiedlichen Orten ab. Durch die hierbei immer auftretenden räumlichen Überschneidungen koppeln die einzelnen Gleichungen für die Ladungsträgerinversion miteinander:

$$
\begin{aligned}
\frac{\mathrm{d}}{\mathrm{d} t} E_{(m)}(t)= & {\left[\frac{i \alpha}{2} G\left(N_{(m)}, \omega_{m}\right)+\frac{1}{2}\left(G\left(N_{(m)}, \omega_{m}\right)-\frac{1}{\tau_{p}}\right)\right] E_{(m)}(t) } \\
& +\kappa_{m} E_{(m)}(t-\tau) e^{-i \omega_{m} \tau} \\
\frac{\mathrm{d}}{\mathrm{d} t} N_{(m)}(t)= & J-\gamma N_{(m)}(t)-\sum_{n} G\left(N_{(m)}, \omega_{m}\right) \beta_{n m}\left|E_{(n)}(t)\right|^{2}
\end{aligned}
$$

Diese Kopplung wird durch $\beta_{n m}$ vermittelt, es gilt $\beta_{n n}=1$. Im Kapitel [6] wird das erstere Modell zur Anwendung kommen. 


\subsection{Modellierung der Dynamik der Intensitätssprünge}

Bisher wurden nur wenige Vorschläge zur Modellierung der Dynamik der Intensitätssprünge publiziert. DE TOMAsi et al. [15] erweiterten das von MøRK et al. 1988 [48] verwendete Modell zur Beschreibung der LFF-Dynamik um mehrfache Reflexionen innerhalb des externen Resonators. Die numerischen Ergebnisse zeigte gute Übereinstimmung und auch die Asymmetrie des Ausgangssignals zwischen den beiden Laserfacetten.

Das von mir entwickelte Modell basiert auf dem LK-Modell und verwendet Mehrfachreflexionen innerhalb des externen Resonators. Mit der Gleichung (3.2) für die Rückkopplungsstärke lässt sich ein konsistentes Modell aufstellen, welches für geringe Pumpströme und geringe Reflektivität des Spiegels LFF-Dynamik und für hohe Pumpströme und hohe Reflektivität des Spiegels LFJ-Dynamik zeigt. Der Bereich des Pumpstromes $p$, indem die LFF-Dynamik auftritt ist jedoch extrem klein, wesentlich kleiner als im Experiment. Darüberhinaus konnte mit dem so bestimmten $\kappa$ bisher keine Antisynchronisation numerisch simuliert werden.

Diese Probleme ließen sich dadurch umgehen, dass unabhängig von Gleichung (3.2) für LFF-Dynamik und LFJ-Dynamik je eigenes $\kappa$ gewählt wurde.

$$
\begin{aligned}
\frac{\mathrm{d}}{\mathrm{d} t} E(t) & =\left[\frac{i \alpha}{2} G(N)+\frac{1}{2}\left(G(N)-\frac{1}{\tau_{p}}\right)\right] E(t)+\kappa A(t-\tau) e^{-i \omega_{0} \tau} \\
A(t) & =E(t)+\sum_{m=1}^{\infty}\left(r_{2} r_{3} e^{-i \omega_{0} \tau}\right)^{m} E(t-m \tau)
\end{aligned}
$$

Die Feldgleichung stimmt bis auf die Einkopplung des Lichtes des externen Resonators mit dem LK-Modell überein. Hier wird $A(t-\tau)$ eingekoppelt, welches außer dem ersten Umlauf des Feldes im externen Resonator auch noch Mehrfachreflexionen beinhaltet (Gleichung (3.16) ). Der hier auftretenden Phasenfaktor $e^{-i \omega_{0} \tau}$ ist im Gegensatz zum ursprünglichen LK-Modell sehr wichtig und nur für eine genau abgestimmte Phasenbeziehung entstehen die Intensitätssprünge2. Die Materiegleichung ist identisch mit der des normalen Diodenlasers (2.31).

Wie aus der Abbildung 3.8 ersichtlich ist, beschreibt $E(t)$ das aus dem Laser emittierte elektrische Feld, dass aus beiden Facetten austritt. Auf der Seite des externen

\footnotetext{
${ }^{2}$ Es muss in der Numerik $e^{-i \omega_{0} \tau} \approx-1$ gelten. Die experimentellen Schwierigkeiten die LFJDynamik zu finden, könnten hiermit zusammenhängen. Es sei noch angemerkt, dass die in dem Phasenfaktor auftretenden Laserfrequenz $\omega_{0}$ lediglich eine Vereinfachung darstellt, da diese durch die Dynamik leicht variiert wird.
} 
Kap. 3 Halbleiterlaser mit optischer Rückkopplung

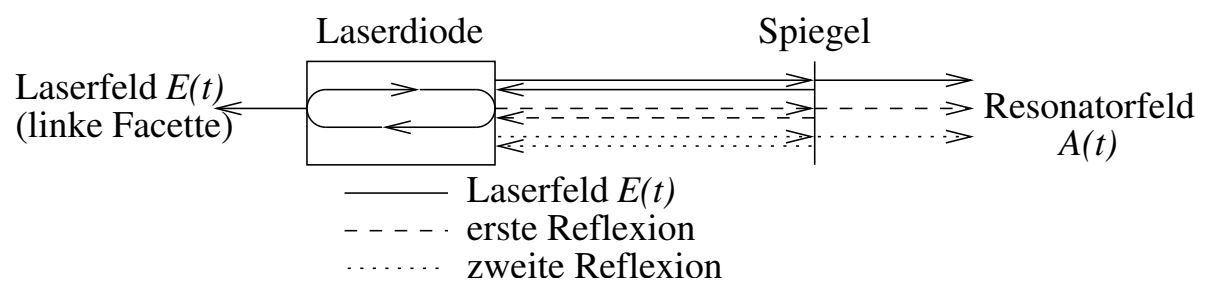

Abbildung 3.8: Aufbau zur Erzeugung der Intensitätssprünge.

Resonators muss jedoch noch das im externen Resonator aufgebaute Feld hinzuaddiert werden. $A(t)$ besteht genau aus diesen beiden Teilen und beschreibt demnach das Feld, wie es an der dem externen Resonator zugewandten Laserfacette, knapp außerhalb des Lasers, anzutreffen ist. Im weiteren bezeichne ich $E(t)$ als Laserfeld und $A(t)$ als (externes) Resonatorfeld. Abbildung 3.9 zeigt die Ergebnisse der numerische Simulation mit diesem Modell. Es sind klar die Intensitätssprünge sowie die Asymmetrie zwischen den Intensitäten beider Laserfacetten zu erkennen. Das Feld innerhalb des externen Resonators scheint also den Intensitätseinbruch des Laserfeldes mehr als kompensieren zu können.
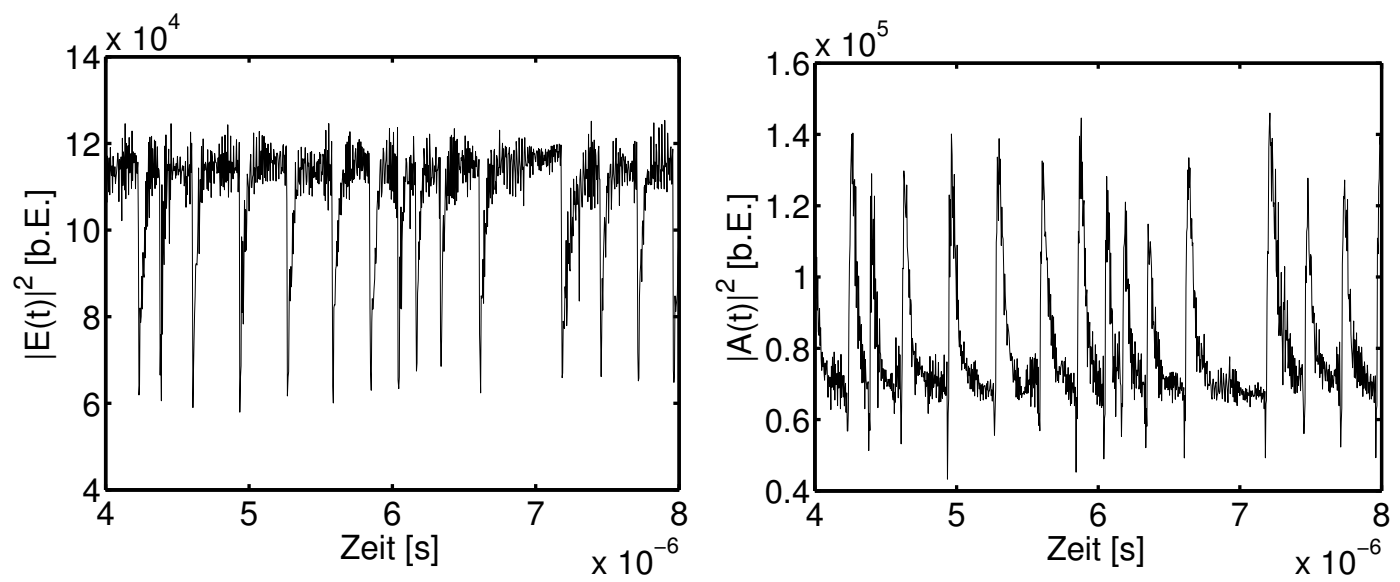

\begin{abstract}
Abbildung 3.9: Numerische Simulation des Intensitätsverlaufs der LFJDynamik. Links die Intensität des Laserfelds $|E(t)|^{2}$ an der linken Laserfacette gemäß Abb. 3.8 rechts die Intensität des Resonatorfelds $|A(t)|^{2}$ auf der Seite des externen Resonators.
\end{abstract}

Leider ist es bei den verwendeten Laserdioden nicht möglich beide Laserfacetten zu beobachten. Bei diesen kommerziell erhältlichen Typen wird das Licht der nicht nach außen sichtbaren Laserfacette auf einen Photodetektor geleitet. Diese s.g. Monitordiode dient hauptsächlich der Funktionskontrolle und besitzt eine viel zu geringe Bandbreite, um die hier beschriebene Dynamik auflösen zu können. Aus diesem Grund kann experimentell nur die Laserfacette auf der Seite des externen Resonators beobachtet 
werden.

Eine genaue Betrachtung der numerischen Dynamik in Abbildung [3.10 zeigt, dass auch auf Seiten des externen Resonators ein Intensitätseinbruch stattfindet - zeitgleich mit dem Laserfeld. Da jedoch die Intensität des Resonatorfeldes vor dem Einbruch geringer war sowie die Intensität danach wesentlich schneller und höher ansteigt, ist der Intensitätseinbruch wesentlich unscheinbarer als der des Laserfeldes.

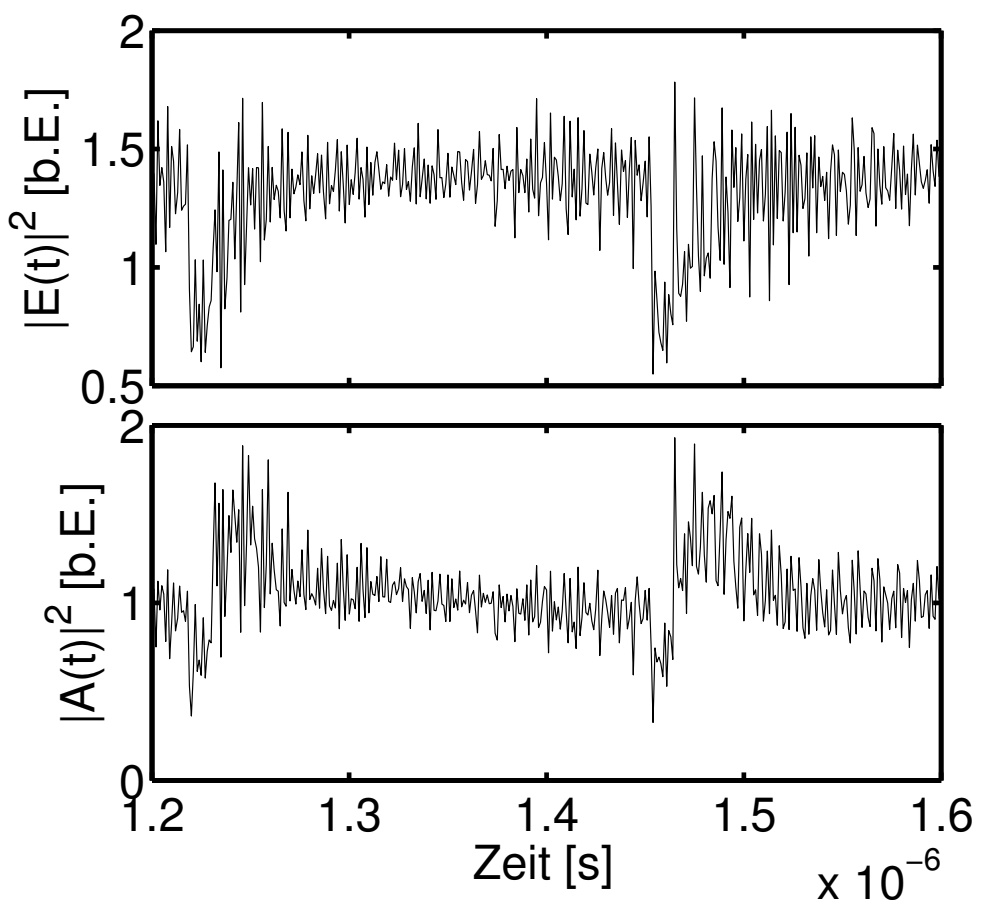

Abbildung 3.10: Genauere Betrachtung der Intensität des Laserfeldes $|E(t)|^{2}$ und des Resonatorsfeldes $|A(t)|^{2}$ bei einem Intensitätseinbruch (numerische Simulation).

Experimentelle Messungen des Resonatorfeldes in Abb. 3.11 zeigen genau diesen sehr kurzen Intensitätseinbruch kurz vor dem starken Anstieg der Intensität sehr gut. Auch in den experimentellen Messungen von PAN et al. [51] ist dieser Einbruch schwach zu erkennen. 


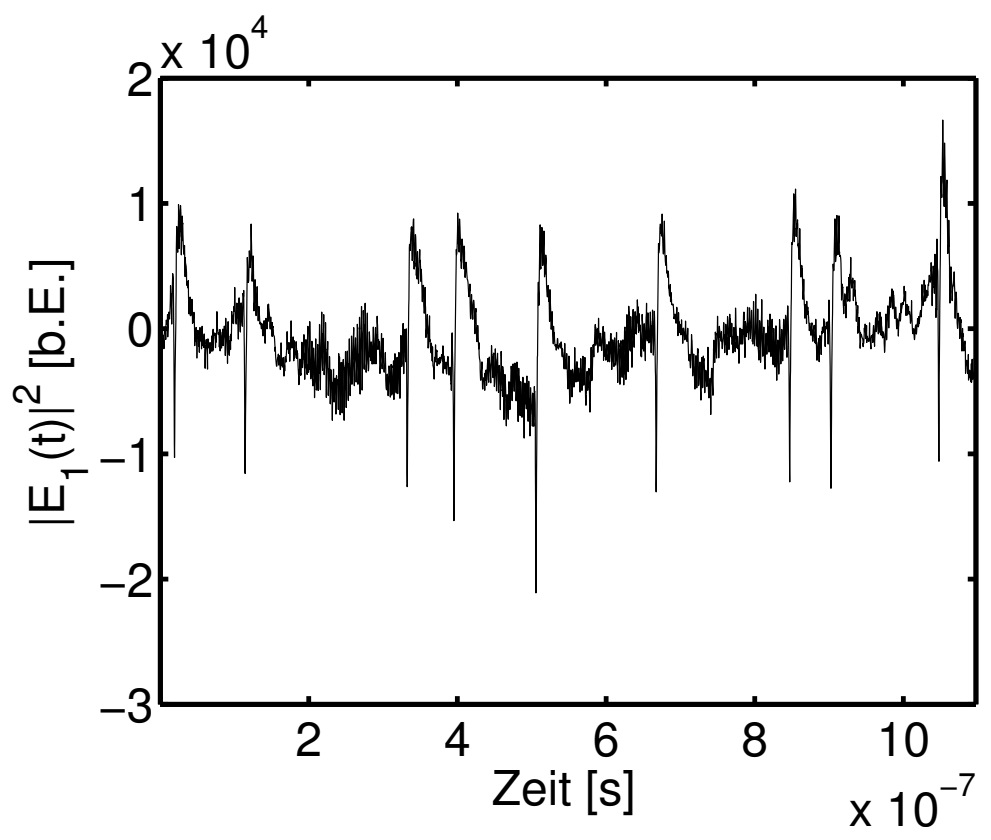

Abbildung 3.11: Experimentelle Messung des Intensitätseinbruchs des Resonatorfeldes kurz von dem starken Anstieg der Intensität bei der LFJ-Dynamik. 


\section{Kapitel 4}

\section{Synchronisation}

Die erste wissenschaftliche Untersuchung zur Synchronisation gekoppelter dynamischer Systeme datiert zurück bis in das 17. Jahrhundert [34]. Der niederländische Physiker ChristiaAn Huygens beobachtete 1673 bei zwei an einem Balken befestigten Pendeluhren gegenphasig synchronisierte Ausschläge der Pendel. Gut 250 Jahre später zeigten VAN DER POL und VAN DER MARK 1926 [75] die Synchronisation von Röhrenoszillatoren durch eine schwache sinusförmige Anregung. Theoretisch erklärt wurde dieses Phänomen von vAN DER POL ein Jahr später [74].

Synchronisationsphänomene sind seit dieser Zeit auch in der belebten Natur entdeckt worden. Beispielsweise synchronisieren die männlichen Spezies der südamerikanischen Glühwürmchen ihre zur Partnersuche ausgesendeten Lichtblitze miteinander. Bis zu einigen tausend gleichzeig aufblinken Glühwürmchen wurden schon beobachtet 8 . Ein vielen bekanntes Beispiel für Synchronisation kann beim Klatschen eines größeren Publikums auftreten. Unbewusst kann sich die Klatschperiode einer großen Anzahl von Teilnehmern synchronisieren wodurch ein typisches rhythmisches Klatschgeräusch ausbildet [50].

Bei all diesen Beobachtungen wurden im wesentlichen periodische Vorgänge synchronisiert. Fujisaka und Yamada 1983 [21, 22], Pikovsky 1984 [55] und Afraimovich 1986 [1] konnten jedoch zeigen, dass Synchronisation auch bei chaotischer Dynamik auftreten kann.

Im folgenden Abschnitt soll genauer darauf eingegangen werden, welche Arten von Kopplungen der Systeme zu Synchronisation führen und welche Arten von Synchronisation unterschieden werden. 


\subsection{Kopplung}

Bevor Synchronisation in irgendeiner speziellen Ausprägung zwischen zwei Systemen auftreten kann, muss eine Kopplung beider Systeme vorliegen. Jedes der System sollte autonom sein und zu einer eigenen Dynamik fähig sein. Desweiteren darf die Kopplung zwischen beiden Systemen nicht zu stark sein, da es sich sonst nicht mehr um zwei getrennte Systeme handelt sondern um ein gemeinsames. Der Übergang von gekoppelten Systemen zu einem komplexeren System aufgrund der Koppelstärke ist jedoch in der Literatur nicht exakt definiert.

Im Experiment von Huygens beeinflussten die beiden Pendeluhren sich gegenseitig über leichte Schwingungen des Tragbalkens, an dem sie aufgehängt waren. Eine solche bidirektionale Kopplung wurde auch später in den Veröffentlichungen von FuJISAKA und YAMADA in ihren numerischen Rechnungen zur Synchronisation des Lorenzsystems verwendet.

Auch möglich ist die unidirektionale Kopplung zweier Systeme, bei der lediglich eines der beiden Systeme, das Empfangssystem (engl. response system), von dem anderen, dem treibenden System (engl. drive system), beeinflusst wird. PECORA and CARrol beschrieben 1990 [53] eine Methode zur Konstruktion solcher unidirektional gekoppelten Systeme sowie eine faszinierende Anwendung: Die sichere Verschlüsselung einer Kommunikation durch Verwendung eines chaotischen Trägers. Durch diese Motivation angespornt, aber auch durch die Analysemöglichkeiten der modernen digitalen Messtechnik wurde in den 90er Jahren des letzten Jahrhunderts diese unidirektional gekoppelten Systeme sehr intensiv untersucht.

Die eben erwähnte Methode zur Konstruktion unidirektional gekoppelter Systeme konnte von KocAREv und PARLitz 1995 [39] zur Aktiv-Passiv-Zerlegung (engl. activepassive decomposition - APD) verallgemeinert werden. Auch die von FUJISAKA und YAMADA verwendete dissipative Kopplung lässt sich im Rahmen der APD beschreiben.

Es wurden im Laufe der Zeit viele verschiedene Kopplungen beschrieben. Eine der wichtigsten ist wohl die dissipative Kopplung, die dem Empfangssystem einen Dissipationsterm hinzufügt. Dieser Dissipationsterm verschwindet für den Fall der Synchronisation. Die globale Kopplung ist beispielsweise bei der multimodalen Lasergleichung realisiert. Alle Feldgleichungen wirken gemeinsam auf die Ladungsträgerinversion, welche auf jede einzelne Feldgleichung zurückwirkt. Die einzelnen Feldgleichungen sind hier indirekt global gekoppelt.

Die optische Kopplung der Laser lässt sich nicht auf eines dieser einfachen Kopplungsschematas zurückführen, da hierbei dauerhaft das Licht des treibenden Lasers in den 
Empfangslaser injiziert wird. Diese Kopplung ist beispielsweise nicht dissipativ.

\subsection{Synchronisationsarten}

Wie der Begriff Synchronisation selber durch seine aus dem griechischen stammenden Bestandteile syn für gemeinsam, gleich und chronos für Zeit bereits andeutet, zeigen synchronisierte Systeme etwas gemeinsam zur selben Zeit. Dieser etymologischen Bedeutung des Wortes am nächsten kommt die Definition von Brown et al. [9], bei der der Abstand einer exakt messbare Eigenschaft jedes der beteiligten Systeme mittels einer Norm bestimmt wird. Verschwindet dieser Abstand so gelten die Systeme als synchronisiert bezüglich dieser Eigenschaft.

Im letzten beiden Dekaden wurden sehr viele unterschiedliche Synchronisationarten entdeckt und definiert. Ich möchte im folgenden einige dieser Synchronisationsarten im einzelnen vorstellen, ohne die obige möglicherweise sehr elegante, aber unanschauliche Definition zu verwenden.

\subsubsection{Identische Synchronisation}

Eine der offensichtlichsten Synchronisationsarten ist die identische Synchronisation, die definitionsgemäß nur zwischen identischen Systemen auftreten kann. Die beiden Systeme haben die Trajektorien $\boldsymbol{x}(t)$ und $\boldsymbol{y}(t)$. Damit die Systeme identisch synchronisieren, muss es eine nicht leere Menge von Anfangswerten $M \subset \mathbb{R}^{2 d}$ der Trajektorien des gemeinsamen Systems $(\boldsymbol{x}, \boldsymbol{y})$ geben, sodass für $\left(\boldsymbol{x}\left(t_{0}\right), \boldsymbol{y}\left(t_{0}\right)\right) \in M$ gilt:

$$
\lim _{t \leftrightarrow \infty}\|\boldsymbol{x}(t)-\boldsymbol{y}(t)\|=\mathbf{0}
$$

Das bedeutet, dass sich die Trajektorien annähern und dann zeitlich und räumlich identische Dynamik zeigen. Das Faszinierende war nun bei der Arbeit von FuJISAKA et al. 1983 [21] die Erkenntnis, dass für chaotische Systeme trotz der bekannten empfindlichen Abhängigkeit von den Anfangsbedingungen die Menge $M$ sehr groß sein kann. Das bedeutet, dass ihre Trajektorien unabhängig davon in welchem Zustand beide Systeme starten asymptotisch konvergieren.

Für die numerische und experimentelle Realisierung wird meistens die sehr hohe Anforderung des verschwindenden Abstands der Trajektorien in (4.1) durch eine schwächere Bedingung || $\boldsymbol{x}(t)-\boldsymbol{y}(t) \|<\varepsilon, \forall t$ ersetzt. Das $\varepsilon$ kann hierbei als Maß für die Güte der Synchronisation angesehen werden. In dieser Arbeit verwende ich den Begriff „Synchronisation"im Sinne von identischer Synchronisation. 


\subsubsection{Antisynchronisation}

Diese Synchronisationsart wird häufig synonym mit Antiphasensynchronisation verwenden. In dem Sinn, indem ich sie verwende stimmt dies jedoch nur für ganz bestimmten Eigenschaften der Schwingungsformen der beobachteten Größe. Dies wird im Abschnitt Phasensynchronisation näher erläutert.

Die Vorsilbe Anti in diesem Begriff soll nicht „das Gegenteil von“ bedeuten, sondern sich auf die antikorrelierten Messgrößen der Systeme beziehen. Sind zwei Systeme vollständig antisynchronisierbar so ergibt sich statt der Gleichung (4.1) die Bedingung

$$
\lim _{t \leftrightarrow \infty}\|\boldsymbol{x}(t)+\boldsymbol{y}(t)\|=\mathbf{0}
$$

In dieser Arbeit und meinen Veröffentlichungen [81, 82, 83, 84] wird eine schwächere Form der Antisynchronisation verwendet, bei der lediglich ein Messwert der Systeme diese Art der Synchronisation aufweist:

$$
\lim _{t \leftrightarrow \infty}\|h(\boldsymbol{x}(t))+h(\boldsymbol{y}(t))\|<\varepsilon
$$

Die Funktion $h(\cdot)$ bildet hierbei den Phasenraum auf die Messgröße ab. Speziell in experimentellen Veröffentlichungen in den letzten Jahren wurde diese Art der Synchronisation auch als Inverse Synchronisation bezeichnet [58, 62, 63].

Es gibt viele Beispiele für diese Form der Synchronisation, auch wenn sie in der Literatur häufig als Antiphasensynchronisation bezeichnet wird [10, 38, 73.

\subsubsection{Phasensynchronisation}

Intuitiv ist diese Art der Synchronisation schnell beschrieben. Schwächer als die Bedingung für die identische Synchronisation oder die Antisynchronisation wird bei der Phasensynchronisation nur die Phase des Signals in der Synchronisationsbedingung verwendet: Es muss zwischen zwei gekoppelten Systemen mit den Phasen $\phi_{1}(t), \phi_{2}(t)$ eine konstante Phasenbeziehung vorliegen

$$
\Delta \phi=n \phi_{1}-m \phi_{2}=\text { const. } \quad n, m \in \mathbb{N}
$$

Für periodische Systeme konnte bereits VAN DER POL 1926 [74, 75] an den bereits erwähnten Röhrenoszillatoren Phasensynchronisation nachweisen. Das Auftreten von höheren Harmonischen als auch von Subharmonischen ließen sich so als Synchronisationsphänomen erklären. 
Für chaotische Systeme, die nicht direkt eine Phase als dynamische Variable besitzen, kann unter bestimmten Bedingungen eine Phase definiert werden. Rosenblum et al. haben 1996 [59] hierfür das komplexe analytische Signal

$$
\psi(t)=s(t)+\frac{i}{\pi} \int_{-\infty}^{\infty} \frac{s(\tau)}{t-\tau} d \tau=A(t) e^{i \phi(t)}
$$

verwendet, welches jedem Signal $s(t)$ eine Phase $\phi(t)$ zuordnet. Hierdurch war der Nachweis der Phasensynchronisation an zwei Rösslersystemen möglich. Problematisch wird diese Phasendefinition bei verschwindendem Betrag $A(t)$, da dann $\phi(t)$ nicht mehr wohldefiniert ist. Da sich die in Gleichung (4.4) beschriebene Synchronisationsbedingung nur schlecht auf chaotische Phasen anwenden lässt, wurde sie etwas vereinfacht:

$$
\left|m \phi_{1}-n \phi_{2}\right|<\text { const. } \quad \forall t>t_{0}
$$

In vielen Veröffentlichungen wird der Begriff der Antiphasensynchronisation verwendet. Gemeint ist damit ein Spezialfall der Phasensynchronisation mit der Synchronisationbedingung:

$$
\left|\phi_{1}-\phi_{2}\right| \approx \pi
$$

Beispiel hierfür ist die von Huygens bei seinen Pendeluhren beobachtete Synchronisation. In einigen Veröffentlichungen wird als Bedingung für Antiphasensynchronisation auch $x(t)=-y(t)$ verwendet [73], obwohl hierbei nicht nur die Phasen sondern auch die Amplituden in die Synchronisationsbedingung eingehen. Mit dieser Bedingung lässt sich vielmehr Antisynchronisation beschreiben.

\subsection{Stabilität}

Betrachtet man die beiden d-dimensionalen, synchronisierten Systeme als ein gemeinsames nichtlineares System im 2d-dimensionalen Phasenraum, so liegen die Aufenthaltspunkte des Gesamtsystems für den beispielhaften Fall der identischen Synchronisation auf einer Hyperebene:

$$
M_{\text {syn }}=\left\{(\boldsymbol{x}, \boldsymbol{y}) \in \mathbb{R}^{2 d} \mid \boldsymbol{x} \equiv \boldsymbol{y}\right\}
$$

Im allgemeinen Fall für beliebige Synchronisationsarten handelt es sich um eine Mannigfaltigkeit, die Synchronisationsmannigfaltigkeit. In Abbildung 4.1 ist diese Mannigfaltigkeit am Beispiel der identischen Synchronisation für zwei beliebige Komponenten $x_{i}$ und $y_{i}$ blau dargestellt. Diese Synchronisationsmannigfaltigkeit entscheidet über die 

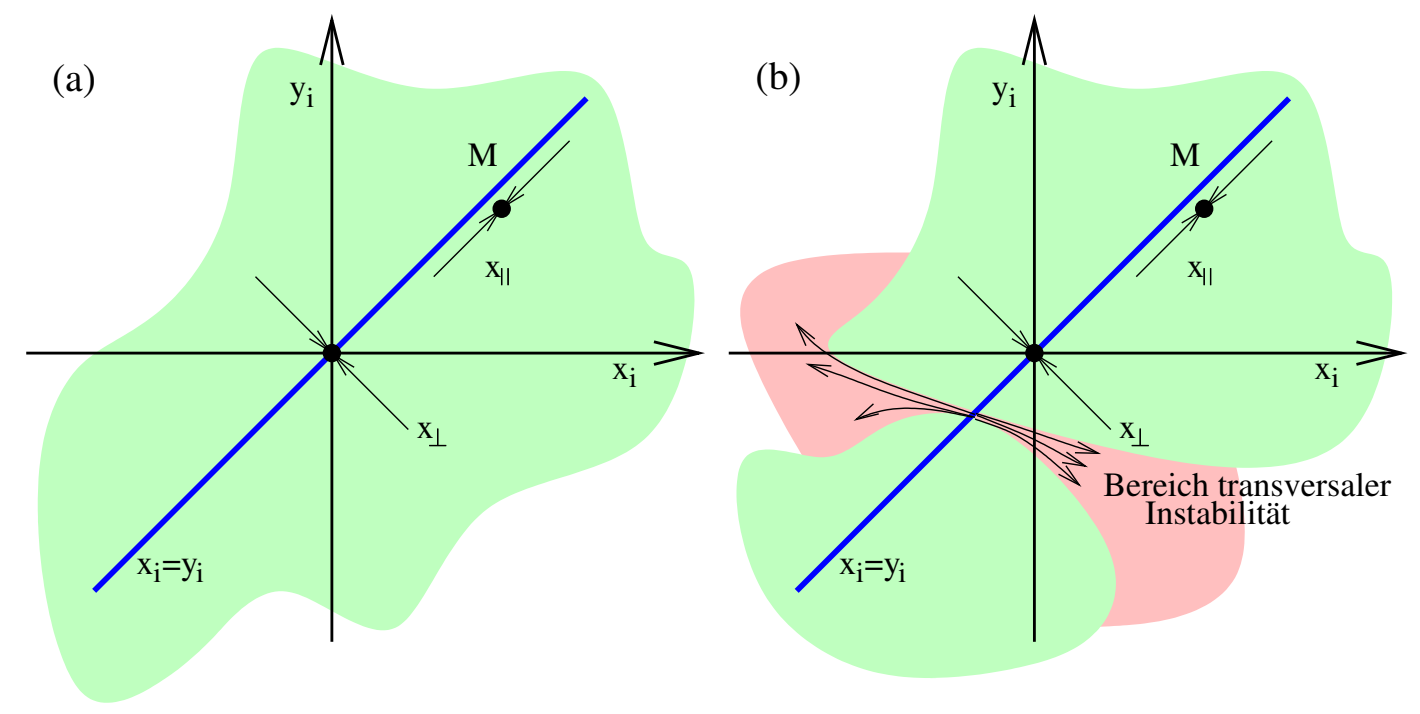

\begin{abstract}
Abbildung 4.1: Synchronisationsmannigfaltigkeit am Beispiel der identischen Synchronisation für die ausgewählten Komponenten $x_{i}$ und $y_{i}$. (a) stabile Synchronisationsmannigfaltigkeit, (b) Synchronisationsmannigfaltigkeit mit transversaler Instabilität.
\end{abstract}

Stabilität der Synchronisation. Sie muss asymptotisch stabil, d.h. mathematisch stabil und attraktiv sein. Um dies zu untersuchen wird das Konzept der transversalen Lyapunovexponenten 1 verwendet. Diese Lyapunovexponenten beschreiben die Expansion der Trajektorien des gemeinsamen Systems transversal zur Synchronisationsmannigfaltigkeit. Sind sie in der Nähe der Synchronisationsmannigfaltigkeit, wie in Abbildung 4.17 durch die grüne Fläche angedeutet, negativ, konvergiert jede Trajektorie auf die Mannigfaltigkeit. Die Synchronisation der beiden Einzelsysteme gilt dann als stabil bzw. robust.

In vielen Systemen, u. A. auch in dem in dieser Arbeit untersuchten Lasern mit optischer Rückkopplung, kommt es zu transversalen Instabilitäten. Eine Trajektorie wird dort transversal von der Mannigfaltigkeit abgestoßen (der rote Bereich in Abb. 4.1b), die beiden Einzelsysteme desynchronisieren. Wenn der abstoßende Bereich klein ist, wird die divergierende Trajektorie jedoch bald wieder von der Mannigfaltigkeit angezogen. In diesem Fall ist die Synchronisationsmannigfaltigkeit nur im Mittel asymptotisch stabil.

\footnotetext{
${ }^{1}$ je nach Quelle sind auch andere Namen wie konditionale Lyapunovexponenten 36 oder sublyapunov exponents [53] geläufig.
} 


\subsection{Messung der Synchronisation}

Zur Bestimmung der Synchronisationsart und Qualität zwischen zwei gekoppelten Systemen im Experiment haben sich verschiedene Mechanismen etabliert, die ich im Folgenden kurz erläutern möchte. Ich beschränke mich hierbei darauf, lediglich skalare Zeitreihen für jedes System zu betrachten, da dies der experimentellen Situation später entspricht.

\subsubsection{Messung der identischen Synchronisation}

Zur Überprüfung der Zeitreihen $\left\{x_{1}, \ldots, x_{N}\right\},\left\{y_{1}, \ldots, y_{N}\right\}$ auf identische Synchronisation lässt sich im einfachsten Fall die normierte Kreuzkorrelation

$$
C_{X Y}(\tau)=\frac{\Gamma_{X Y}(\tau)}{\Gamma_{X X}(\tau) \Gamma_{Y Y}(\tau)}
$$

berechnen. Hierbei bezeichnet $\Gamma_{X Y}$ die Kovarianzfunktion

$$
\Gamma_{X Y}=\frac{1}{N-\tau} \sum_{i=1}^{N-\tau}\left(x_{i}-\bar{x}\right)\left(y_{i}-\bar{y}\right)
$$

und $N$ die Länge der Zeitreihe, $\tau$ die Zeitverschiebung und $\bar{x}, \bar{y}$ den Mittelwerten der Zeitreihen $x_{i}$ bzw. $y_{i}$.

Für ausreichend komplexe und wenig periodische Signale, wie sie bei den hier untersuchten Lasern vorliegen, ergibt sich nur bei einer bestimmten Zeitverschiebung $\tau_{c}$ eine hohe positive Korrelation. Diese Korrelation ist auch für nicht perfekte Synchronisation, wie sie experimentell häufig anzutreffen ist, gut zu identifizieren (Abb. 4.2). Die Qualität der Synchronisation wird durch den Abstand des Kreuzkorrelationskoeffizienten $C_{X Y}\left(\tau_{c}\right)$ zur 1 definiert2. Bei verschwindendem Abstand besteht perfekte Synchronisation, in der Nähe von 0 ist keine Ähnlichkeit und demnach Synchronisation erkennbar. Das $\tau_{c}$ wird mit der Kopplungsverzögerung identifiziert. Bei komplexeren Systemen kann dieses $\tau_{c}$ auch von den Parametern des Systems abhängen.

\footnotetext{
${ }^{2}$ Eine Kreuzkorrelationskoeffizient von 1 ist strenggenommen nicht ausreichend für die Detektion perfekter identische Synchronisation, da die Skalierung der Signale durch die Normierung keine Rolle spielt. In der Definition der identischen Synchronisation (4.1) müssen beide Systeme mit identischen Signalen vorliegen, eine Skalierung ist hier nicht zulässig. Im Experiment spielt diese Problematik nur eine untergeordneter Rolle, da die Skalierungen der Signale von dem gewählten Aufbau und den Detektoren abhängen. Die „wahre" Skalierung der Signale ist häufig nicht bestimmbar.
} 

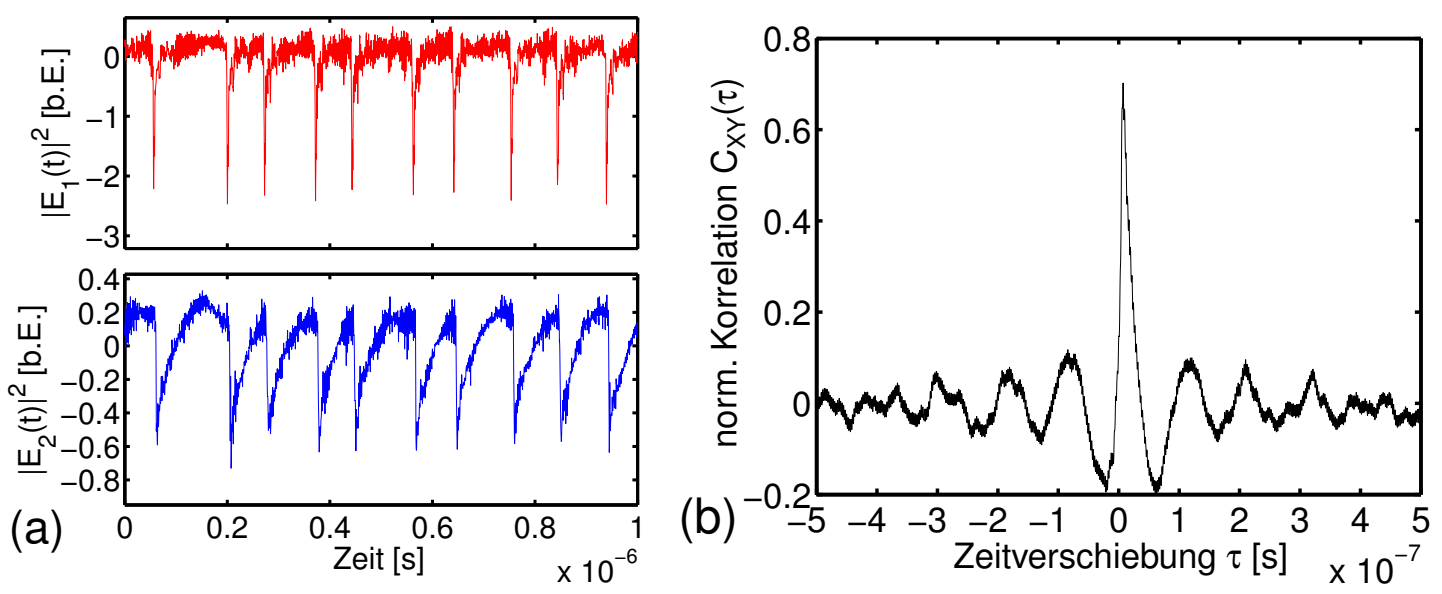

Abbildung 4.2: Kreuzkorrelation als Maß für identische Synchronisation, hier dargestellt bei nur mäßiger Synchronisationsqualität. (a) Zeitreihe der Intensität beider Laser, (b) Kreuzkorrelation.

Bei der experimentellen Suche nach identischer Synchronisation und bekanntem $\tau_{c}$ bietet sich die Verwendung der XY-Darstellung des Oszilloskops an. Moderne digitale Speicheroszilloskope können die Kopplungsverzögerung $\tau_{c}$ aus den Zeitreihen bereits intern herausrechnen und $x(t)$ gegen $y\left(t-\tau_{c}\right)$ direkt auf dem Bildschirm darstellen. Dadurch lässt sich identische Synchronisation sehr schnell und einfach messen wie in Abbildung 4.3 dargestellt. Die Qualität wird hierbei über die Breite der Punktverteilung entlang der Diagonalen $x=y$ qualitativ bestimmt.

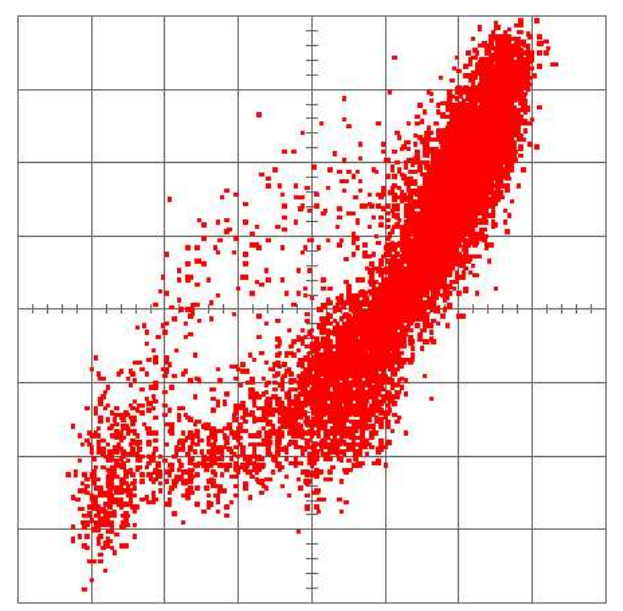

Abbildung 4.3: Messung identischer Synchronisation im Experiment mit der XY-Darstellung des Oszilloskops. 


\subsubsection{Messung der Antisynchronisation}

Da auch bei der Antisynchronisation ein linearer Zusammenhang zwischen den Messwerten beider Zeitreihen besteht, lässt sich auch diese Synchronisationsart gut mit der Kreuzkorrelation aufspüren. Sinngemäß ergibt sich für ein bestimmtes $\tau_{c}$ ein hoher negativer Korrelationskoeffizient (Abb. 4.4). In diesem Fall wird die Synchronisationsqualität durch den Abstand dieses Korrelationskoeffizienten zur -1 bestimmt.
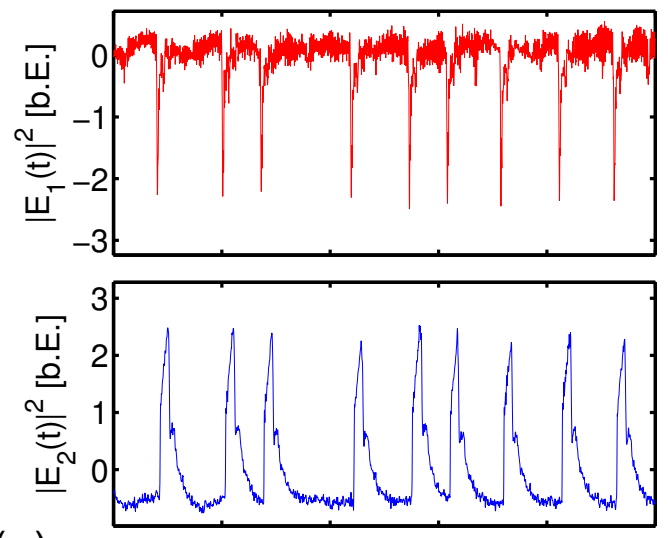

(a)

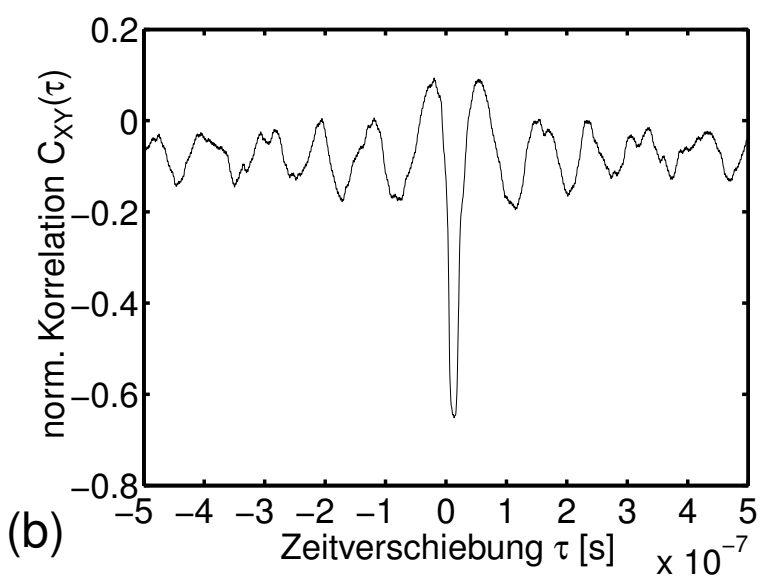

Abbildung 4.4: Kreuzkorrelation zur Bestimmung von Antisynchronisation.

(a) Zeitreihe der Intensität beider Laser, (b) Kreuzkorrelation.

Im Experiment lässt sich diese Art der Synchronisation mit der XY-Darstellung ebenfalls sehr einfach entdecken, da sich die Kurve bei korrekt kompensiertem $\tau_{c}$ entlang der Diagonalen $x=-y$ darstellt (Abbildung 4.5).

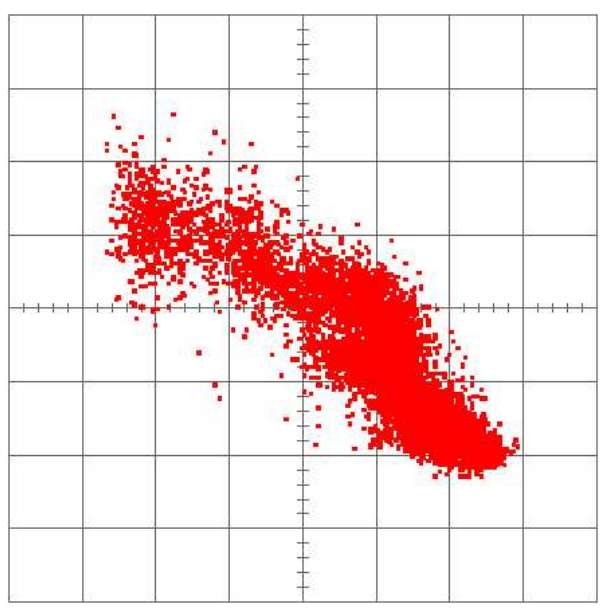

Abbildung 4.5: Antisynchronisation detektiert mit dem Oszilloskop in XY-

Darstellung. 


\subsubsection{Messung der Phasensynchronisation}

Die Phasensynchronisation spielt in vielen Experimenten beispielsweise der Kopplung von Herzschlag und Atmung und auch in den Neurowissenschaften bei der Auswertung von EEG-Daten eine sehr wichtige Rolle. In diesem Zusammenhang wurden viele sehr ausgefeilte Methoden zur Detektion der Phasensynchronisation entwickelt und im Experiment erprobt [54]. Ich möchte hier nur kurz einige grundlegende Beispiele geben, da die Phasensynchronisation nur in den numerischen Simulationen des Lasers detektierbar ist.

Am Beispiel der nach Betrag und Phase aufgespaltenen Lasergleichungen (3.5), lässt sich Phasensynchronisation relativ leicht durch die Auftragung der generalisierten Phasendifferenz

$$
\phi_{n m}=n \phi_{1}-m \phi_{2}
$$

darstellen. Bleibt diese Differenz beschränkt, so sind die beiden Systeme nach der Definition in Gleichung (4.6) phasensynchron. In unmittelbarer Nähe dieses Synchronisationsgebietes bleibt die Differenz aufgrund von Phasensprüngen (engl. phase slip) um Vielfache von $2 \pi$ nicht beschränkt (Abb. 4.6). Zwischen diesen Phasensprünge bleibt die generalisierte Phase jedoch näherungsweise konstant.

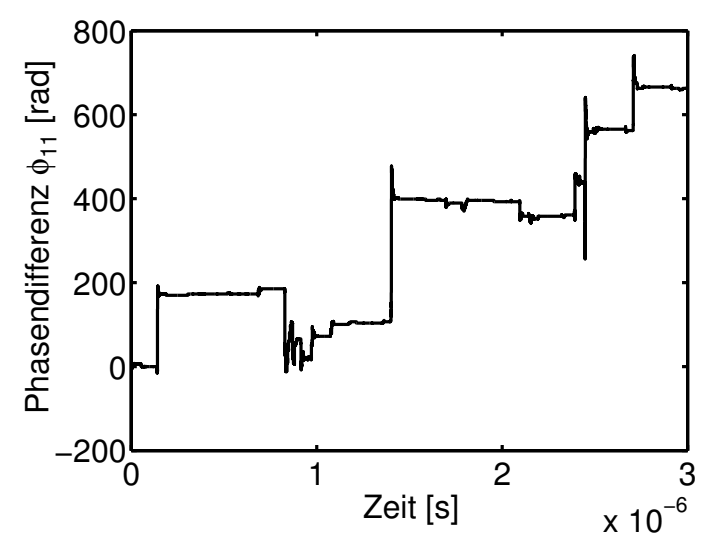

Abbildung 4.6: Auftragung der generalisierten Phasendifferenz $\phi_{11}$ für zwei gekoppelte Lasergleichungen.

In diesem Fall bietet es sich an, die Darstellung der generalisierten Phasendifferenz $\phi_{11}$ Modulo $2 \pi$ als Histogramm aufzutragen [72]. So bekommt man einen Eindruck bei welchen Phasenverschiebungen die Phasedifferenz häufig konstant bleibt (Abb. 4.7).

In dieser Auftragung ist klar erkennbar, dass neben der Phasendifferenz 0 auch die Phasendifferenz $-\pi$ häufig auftritt. Grund hierfür liegt in dem identischen Einfluss der Feldgleichung bei Vorzeichenwechsel. 


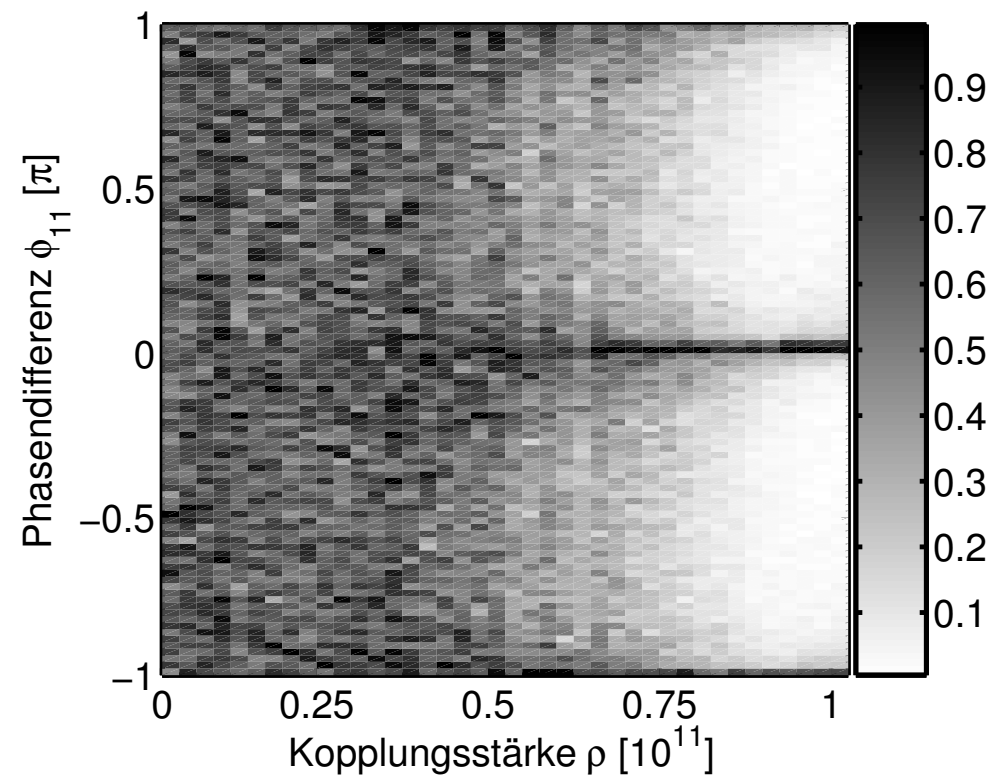

Abbildung 4.7: Darstellung der normierten Phasenhistogramme aufgetragen gegen die Variation des Kopplungsparameters.

Bei einfachen Systemen ohne Phasenvariable, beispielsweise bei einem Rössleroszillator, kann je nach Struktur des Attraktors eine Phase über den Arkus Tangens konstruiert werden [80]. Auch hier kann es jedoch zu dem Problemen der Wohldefiniertheit der Phase bei Nulldurchgang der Trajektorie kommen, ähnlich wie bei der Phasendefinition aus dem analytischen Signal. Für die experimentell aufgenommene Laserdynamik ist aufgrund der Struktur des Signals die Wahrscheinlichkeit des Auftretens dieser Probleme sehr hoch, weshalb diese Phasenkonstruktionen hier nicht sinnvoll sind. 



\section{Kapitel 5}

\section{Experimentelle Synchronisation von Lasern}

\subsection{Historischer Überblick}

Die Motivation von PeCorA und CARrol, synchronisierte chaotische Dynamik als sichere Kommunikationsmethode verwenden zu können [53], verstärkte das Interesse und die Forschungstätigkeit auf diesem Gebiet enorm. Bis zu dem Zeitpunkt dieser Veröffentlichung wurde die chaotische Synchronisation nur numerisch anhand einfacher niedrigdimensionaler Systeme untersucht. Der experimentelle Aufwand für eine Synchronisation von Lasern ist sehr hoch, allein schon auf Grund der Tatsache, dass das physikalische Zusammenspiel der einzelnen Effekte nicht direkt beobachtet werden kann. Auch viele Parameter des Lasers sind kaum zu beeinflussen.

Die erste experimentelle Synchronisation zweier chaotisch fluktuierender Nd:YAG Laser gelang Roy und Thornburg 1994 [60]. Die bidirektionale Kopplung wurde hierbei durch den für beide Laser gemeinsam genutzten Nd:YAG-Kristall erreicht. Durch den räumlichen Abstand der aktiven Kristallbereiche beider Laser konnte die Kopplungsstärke in Form der Beeinflussung der Ladungsträgerinversion eingestellt werden. Ebenfalls 1994 konnten Sugawara et al. 68] zwei chaotisch pulsierende $\mathrm{CO}_{2}$-Laser synchronisieren. Die Kopplung wurde hierbei durch einen vom ersten Laser modulierten sättigbaren Absorber im zweiten System erreicht. Die erste Synchronisation von Laserdioden wurde 1998 von GoEDGEBUER et al. gezeigt [23]. Die Laserdioden produziert aufgrund von elektro-optischer Rückkopplung chaotische Dynamik, die Kopplung wurde hierbei elektrisch durch Pumpstromvariation bewerkstelligt.

1999 konnten Sivaprakasam und Shore [65] erstmals die experimentelle Synchronisation zweier optisch unidirektional gekoppelter Halbleiterlaser mit externem Resonator zeigen. Sie verwendeten in beiden Systemen einen externen Resonator. TAKIGUCHI et al. konnten noch im selben Jahr [70] mit einen ähnlichen Aufbau jedoch ohne externen Resonator im Empfangssystem identische Synchronisation demonstrieren. 
Ein Jahr später konnte ich im Rahmen meiner Diplomarbeit experimentell zwei optisch unidirektional gekoppelte Halbleiterlaser mit externem Resonator sowohl synchronisieren als auch antisynchronisieren [80, 81. Mit dem Begriff der Antisynchronisation beziehe ich mich hierbei auf die schwächere Form, die ich im Abschnitt 4.2.2 in Gleichung (4.3) definiert habe. Die von mir beobachteten physikalischen Größen sind die Intensitäten der Halbleiterlaser, wie sie durch die verwendeten Photodetektoren aufgenommen wurden. Das bedeutet, es kann nur ein entsprechend der Bandbreite der Detektoren tiefpassgefiltertes Signal der wahren Intensitätsfluktuation des Lasers experimentell beobachtet werden. Inwieweit die diesem Signal zugrunde liegende Dynamik ebenfalls antisynchron ist, kann experimentell mit den vorhandenen Mitteln nicht überprüft werden.

Die von mir als Antisynchronisation bezeichnete Synchronisationart wurde kurze Zeit später auch von SivaPraKasam et al. 63] experimentell gemessen. Diese Arbeitsgruppe nannte diese Art der Synchronisation inverse synchronization, da das Empfangssystem mit einem dem treibenden Laser invertierten Intensitätssignal antwortet 1 .

\subsection{Experimenteller Aufbau}

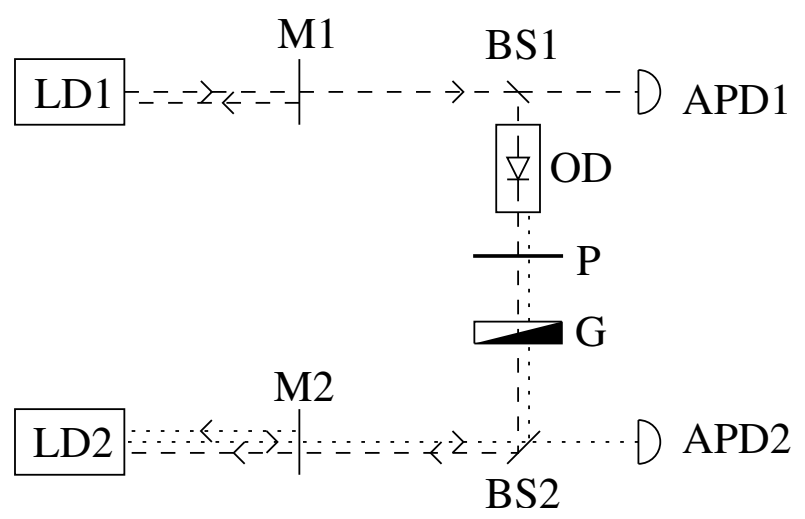

Abbildung 5.1: Experimenteller Aufbau zur Synchronisation zweier Halbleiterlaser.

In Abbildung [5.1]ist der von mir in dieser Arbeit verwendete Aufbau zur Synchronisation zweier Halbleiterlaser skizziert. Der variable Strahlteilen 2 M1 bildete einen externen

${ }^{1}$ Diese Namensdefinition macht so nur im Englischen Sinn, da es im Deutschen einen mathematischen Unterschied der Bedeutung von invers $\left(\propto \frac{1}{x}\right)$ und invertiert $(\propto-x)$ gibt. Im englischen Sprachgebrauch wird hingegen häufig inverse im Sinne von invertiert verwendet.

${ }^{2}$ Hierbei handelt es sich um eine verspiegelte Glasoberfläche mit räumlich unterschiedlicher Reflektivität. 
Resonator für Laser LD1. Das Licht wird durch den Strahlteiler BS1 zum einen dem Photodetektor APD1 zugeführt und zum anderen durch den Faradayisolator OD, den Polarisator P, den Graukeil G und den Strahlteiler BS2 in den zweiten Laser LD2 eingekoppelt. Dieser bildet mit dem variablen Strahlteiler M2 ebenfalls einen externen Resonator und wird über den Photodetektor APD2 beobachtet. Die Rückkopplung des Laserlichtes LD2 durch M2 wurde wahlweise auch dejustiert, um ein Laser ohne externen Resonator zu bilden (dadurch bleibt die Kopplungsstärke aufgrund der Transmission durch den variablen Strahlteiler nahezu konstant).

Durch die Orientierung des Faraday-Isolators bildet LD1 zusammen mit M1 das treibende System, LD2 mit M2 das empfangende. Im weiteren wird der Index 1 für alle experimentellen Parameter und Messgrößen von LD1 bzw. APD1 verwendet sowie Index 2 für die entsprechenden Größen von LD2 bzw. APD2.

Die technischen Bezeichnungen der verwendeten optischen und elektrischen Komponenten finden sich im Anhang B.

\section{Beeinflussbare Parameter}

Bisher habe ich nur von zwei Synchronisationsphänomen gesprochen, die bei der Betrachtung zweier gekoppelter Lasersysteme identifizierbar sind. Aufgrund der Komplexität des Systems sind jedoch auch die beobachteten Phänomene im Experiment wesentlich umfangreicher und weniger gut in Begriffe zu fassen. Diesen Zoo von Phänomenen zu systematisieren ist deswegen nicht zur Gänze möglich, da die dabei zu untersuchenden Parameterbereiche der fast ein Duzend im Experiment veränderbaren Parameter zu umfangreich sind. Die experimentell beeinflussbaren Parameter sind

- Pumpstrom beider Laser $I_{1}, I_{2}$

- Temperatur beider Laser $T_{1}, T_{2}$

- Reflektivitäten der Spiegel in den externen Resonatoren für beide Laser $\kappa_{1}, \kappa_{2}$

- Größen der externen Resonatoren beider Laser $\tau_{1}, \tau_{2}$

- Kopplungsstärke $\rho$ und Kopplungszeit $\tau_{c}$

- Kopplungsart (uni- und bidirektional).

Darüberhinaus ist, wie schon erwähnt, nur ein kleiner Teil der Dynamik des Laserlichtes experimentell messbar: nur Intensitätsfluktuationen bis ca. 200MHz. Weitere 
Einschränkungen ergaben sich durch den von mir verwendeten Aufbau und durch die zur Verfügung stehenden optischen Komponenten.

Für die weitere Untersuchung habe ich folgende Einschränkungen der Parameter vorgenommen:

- Temperaturbereich beider Laser zwischen $10-30^{\circ} \mathrm{C}$ um sowohl Kondenswasserbildung als auch Überhitzung der Laser auszuschließen

- Pumpströme größer als ca. 25mA, da ab hier die Laserschwelle zu erwarten ist

- Größe des externen Resonators ca. $35 \mathrm{~cm}$

- unidirektionale Kopplung.

\subsection{Synchronisation und Antisynchronisation der LFF- Dynamik}
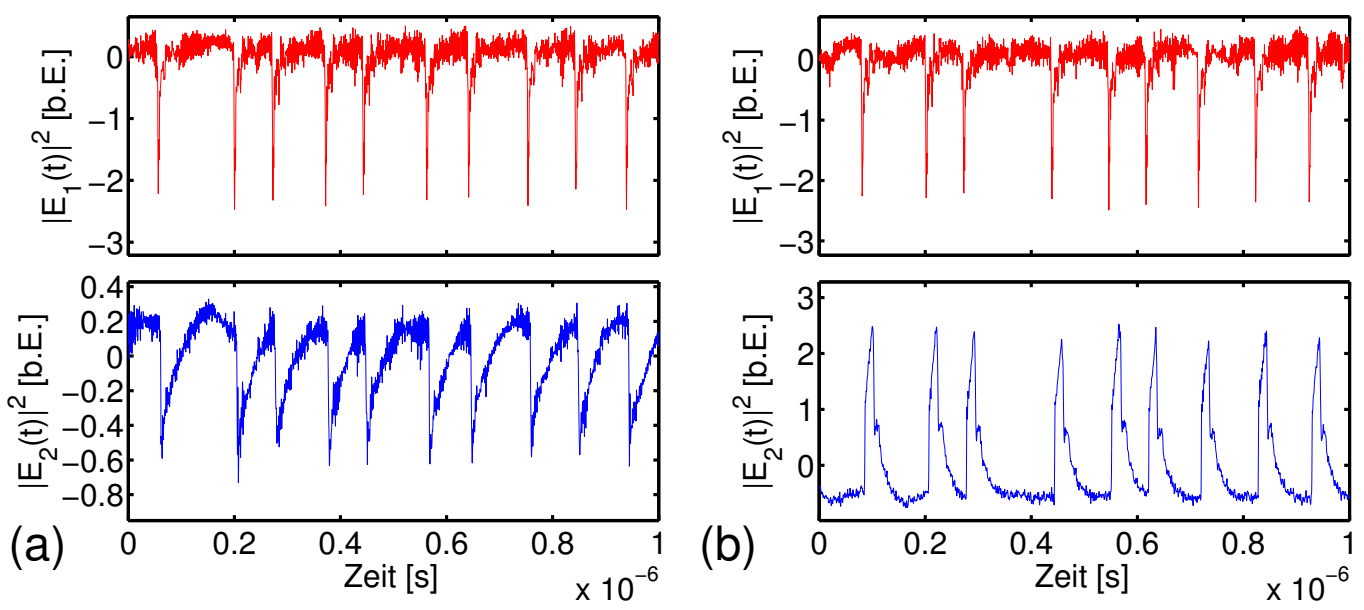
Abbildung 5.2: Experimentelle Synchronisation (a) und Antisynchronisation (b) der LFF-Dynamik zwischen dem treibenden Laser (rote Kurve) und dem empfangenden Laser (blaue Kurve).

In Abbildung [5.2a ist ein Beispiel für die Synchronisation der LFF-Dynamik zu erkennen. Die Intensitätseinbrüche sind zwar genau synchron, der gesamte Intensitätsverlauf ist jedoch nicht exakt gleich, was lediglich zu einem maximalen Kreuzkorrelationskoeffizienten von $C_{X Y} \approx+0.85$ führt. Grund für die nicht perfekt übereinstimmenden Feinstruktur der beiden Intensitätskurven kann eine nicht perfekte Synchronisation 
sein, beispielweise aufgrund von im Experiment nicht beeinflussbaren Parameterunterschieden. Eine andere Erklärung ist die im Experiment durchaus großen Unterschiede im Frequenzverhalten der Photodetektoren. Abbildung $5.2 \mathrm{~b}$ zeigt ein Beispiel für die Antisynchronisation beider Laser. Die Antisynchronisation ist hier ebenfalls nicht perfekt.

\subsubsection{Einfluss der Kopplungsstärke}

Bevor eine genaue Untersuchung des Parameterraums zur Lokalisation der Synchronisations- und Antisynchronisationsbereiche folgt, möchte ich kurz die Auswirkung der Kopplungsstärke $\rho$ bestimmen. In Abbildung $[5.3$ wurde der Kreuzkorrelationskoeffizient mit dem maximalen Betrag farbkodiert für verschiedene Pumpströme des empfangenden Lasers $I_{2}$ und Einkoppelstärken $\rho$ aufgetragen. Der empfangende Laser wurde ohne externen Resonator betrieben. Der verwendete Graukeil besaß einen linearen Transmissionsfaktor von $\approx 0-80 \%$, danach blockierte die Aufnahme des Graukeils die Kopplung vollständig. Die Farbkodierung zeigt hier blau für hohe negative Korrelationen und rot für hohe positive Korrelationen gemäß der Farbtabelle in Abb. C.5. Für nur schwache Korrelation resultiert eine grüne Farbgebung. Wie in den Abschnitten 4.4.1 und 4.4.2 beschrieben, lassen sich rote Bereiche mit (identischer) Synchronisation und blaue Bereiche mit Antisynchronisation identifizieren. Da im folgenden viele farbkodierte Darstellungen verwendet werden, habe ich darauf verzichtet, an jede Auftragung die jeweilige Farbtabelle anzugeben. In Anhang [C sind alle verwendeten Farbtabellen zusammengefasst.

\section{Verwendung des Synchronisationsindexes}

Leider ist diese Auftragung so nicht wirklich sinnvoll, es ergeben sich durch die Wahl des normierten Kreuzkorrelationskoeffizienten zwei Probleme: Zum einen können sich auch für sehr kleine Amplituden des empfangenden Lasersystems hohe Korrelationen ergeben, dies ist wenig intuitiv. Zu erwarten wäre hier eher eine geringe Synchronisationsqualität. Das zweite Problem sind mögliche zufällige Korrelationen bei sehr schwachem Signal des Empfangslasers aufgrund der Diskretisierung des Signals. Alle Laserintensitäten sind mittels Photodetektoren und eines Speicheroszilloskops mit einer Auflösung von 8 Bit aufgezeichnet worden. Bei sehr geringen Intensitäten ergeben sich nur sehr wenige mögliche Werte für das Signal und es kann zu zufälligen Korrelationen kommen. 


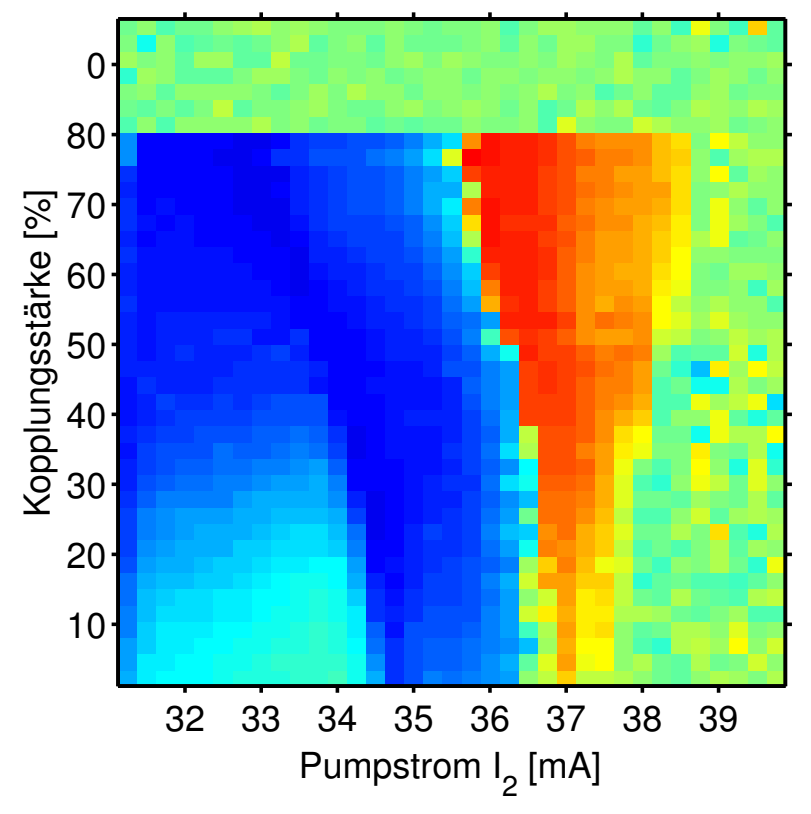

Abbildung 5.3: Auftragung des Kreuzkorrelationskoeffizienten mit dem maximalen Betrag farbkodiert für verschiedene Pumpströme des empfangenden Lasers $I_{2}$ und Kopplungsstärken $\rho . I_{1}=32.5 \mathrm{~mA}, T_{1}=19.4^{\circ} \mathrm{C}, T_{2}=19.2^{\circ} \mathrm{C}$. Kein Resonator im Empfangssystem.

Aus diesen Gründen habe ich einen anderen Wert für die Detektion der Synchronisation verwendet.

$$
S=C_{X Y}\left(\arg \max _{\tau}\left|C_{X Y}(\tau)\right|\right) \cdot\left(\max _{t}(Y)-\min _{t}(Y)\right)
$$

Hierbei sollen $X$ und $Y$ die Zeitreihen der beiden Intensitätssignale sein. Der vorher verwendete Kreuzkorrelationskoeffizient mit dem größten Betrag wird mit der SpitzeSpitze-Amplitude der $Y$-Zeitreihe skaliert (also entsprechend der Größe der Fluktuation). Beide oben beschriebene Probleme sind damit behoben. Dieser Synchronisationsindex $S$ ist in Abbildung 5.4 für Pumpstrom $I_{2}$ und Kopplungsstärke $\rho$ aufgetragen.

Ein weiteres Problem dieser Auftragung liegt direkt in der Verwendung der Kreuzkorrelation, unabhängig von deren Normierung. Für Korrelationskoeffizienten die vom Betrage nach kleiner als eins sind $\left|C_{X Y}(\tau)\right|<1$ spricht man bei der jeweiligen Zeitverschiebung $\tau$ zwischen den Signalen $X$ und $Y$ zwar von (anti-)korrelierten Signalen, die Signalform beider Signale muss jedoch nicht unbedingt ähnlich sein. Um zu überprüfen in wie weit die simple Annahme - blau bedeutet Antisynchronisation und rot bedeutet Synchronisation - zutrifft habe ich für ausgewählte Parameterkombinationen (a, b, c und d in Abbildung 5.4) die Zeitreihen beider Laserintensitäten aufgezeichnet und dargestellt (Abb. 5.5 a, b, c, d). Diese Auftragungen zeigen, dass die Zuordnung 


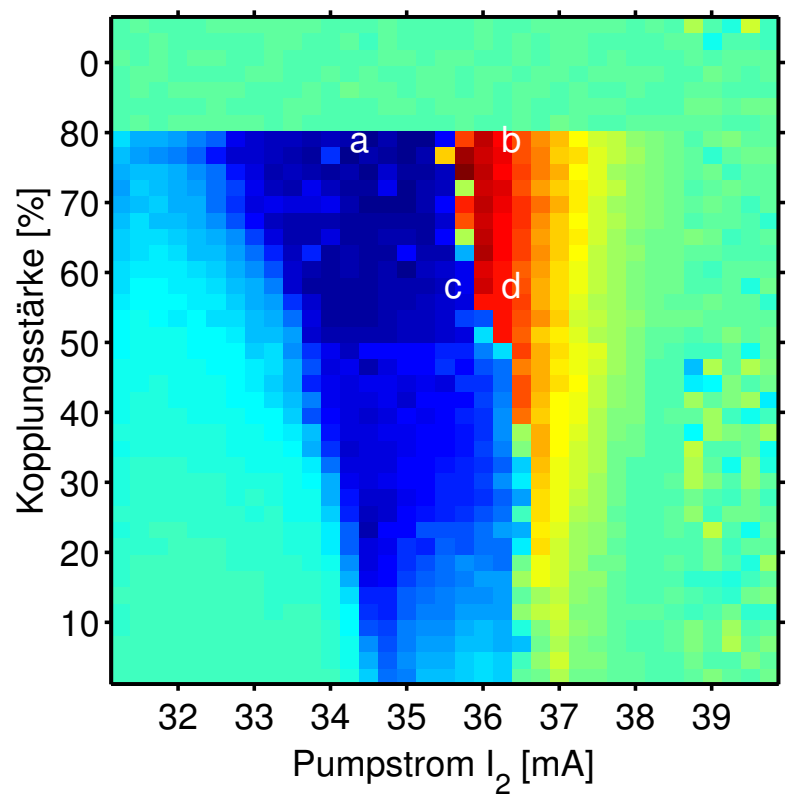

Abbildung 5.4: Auftragung des Synchronisationsindexes für verschiedene Pumpströme des empfangenden Lasers $I_{2}$ und Kopplungsstärken $\rho . I_{1}=$ $32.5 \mathrm{~mA}, T_{1}=19.4^{\circ} \mathrm{C}, T_{2}=19.2^{\circ} \mathrm{C}$.

der Synchronisationsart mit dem in Gleichung (5.1) definierten Synchronisationindex $S$ in diesem Fall gut funktioniert.

Unabhängig von diesen anfänglichen Problemen mit der Auswertung ist in der Auftragung 5.4 sehr schön der Übergang von dem nicht synchronisierten Lasersystem zum synchronisierten System für beide Synchronisationsarten erkennbar. Für den Bereich der Synchronisation ist sehr gut die Verbreiterung des Synchronisationsbereiches durch die Erhöhung der Kopplung zu erkennen (Arnold-Zuge).

\section{Messung mit externem Spiegel im empfangenden System}

Die Messung des Synchronisationsindexes $S$ über dem Diodenstrom $I_{2}$ und der Kopplungsstärke $\rho$ habe ich auch für den Fall mit externem Resonator im empfangenden System aufgenommen (Abbildung [5.6).

Hier sieht die Situation wesentlich unklarer und komplizierter aus als für den rückkopplungsfreien Fall. Eine Arnoldzungenbildung ist nicht erkennbar. Die Zeitreihen der Laserintensitäten (Abb. 5.7 h, b, c, d, e, f, g, h) zeigen hier für die verschiedenen Korrelationsgebiete (Abb. 5.6h, b, c, d, e, f, g, h) teilweise sehr unterschiedliche Signalformen zwischen beiden Lasern, eine grobe Einteilung ist jedoch möglich. Antisynchronisation lässt sich an Punkt (a) und (e) antreffen, dies stimmt auch mit dem 

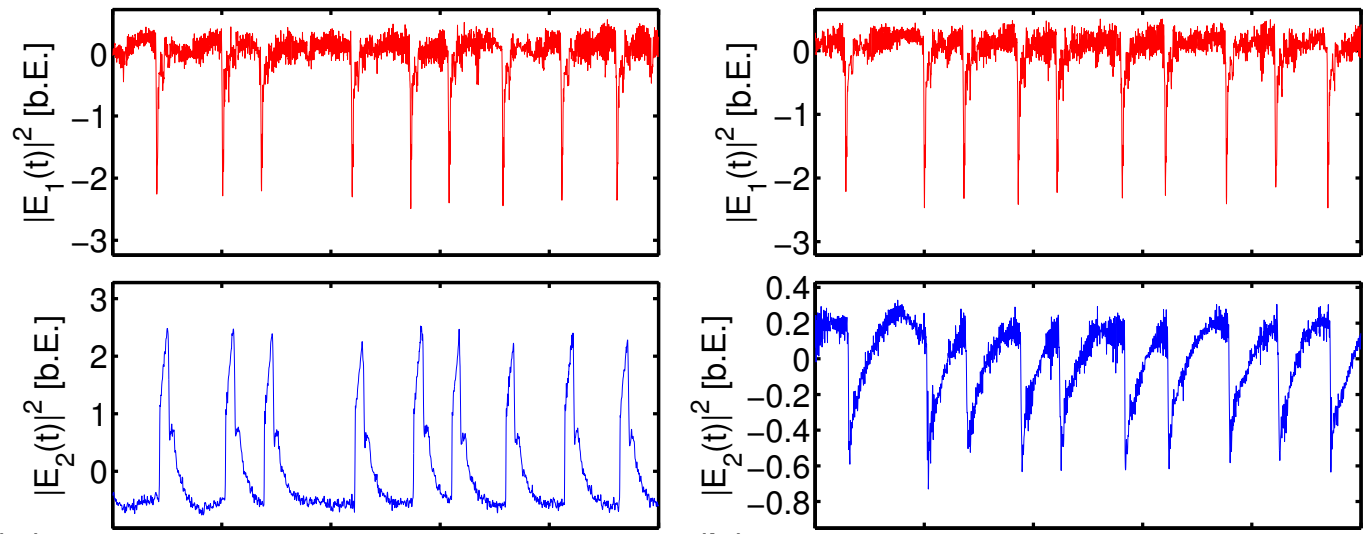

(a)
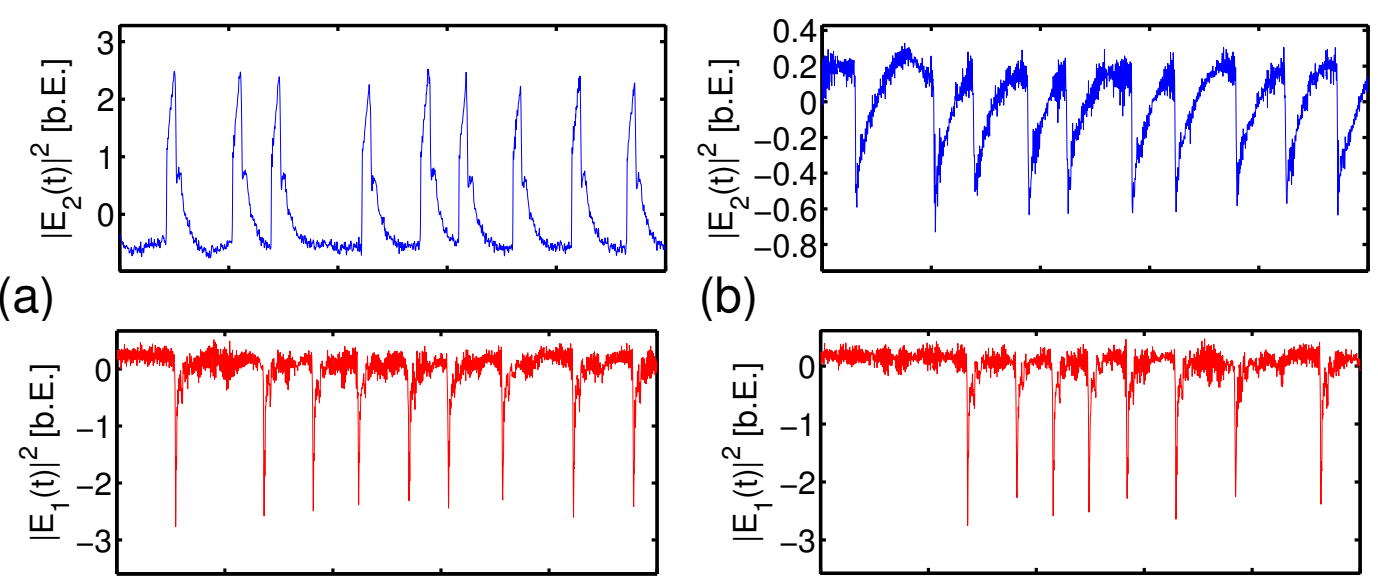

(b)
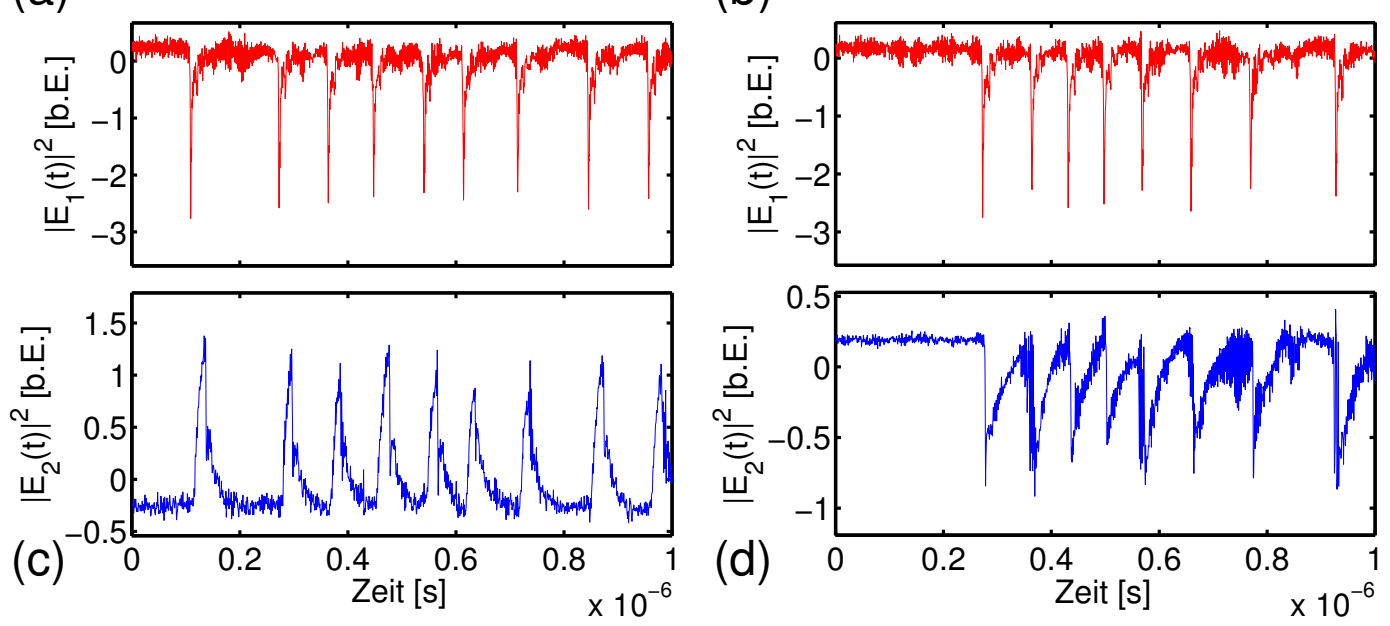

Abbildung 5.5: Exemplarische Aufnahme von Zeitreihen der Laserintensitäten zu verschiedenen Parameterkombinationen aus Kopplungsstärke $\rho$ und Pumpstrom des empfangenden Systems $I_{2}$ gemäß den Punkten (a, b, c und d) aus Abbildung [5.4. 


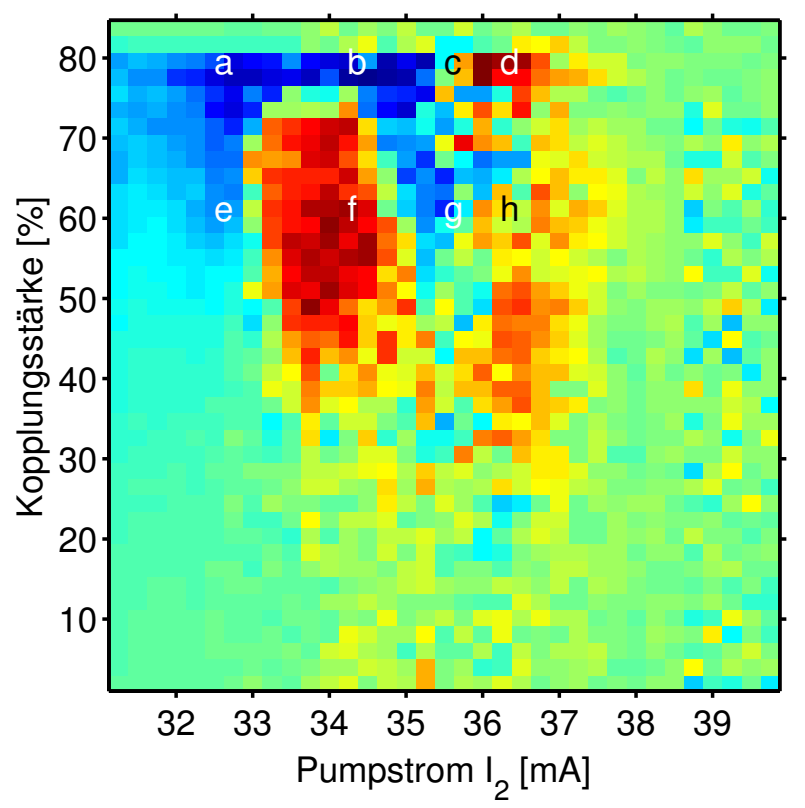

Abbildung 5.6: Auftragung des Synchronisationsindexes für verschiedene Pumpströme des empfangenden Lasers $I_{2}$ und Kopplungsstärken $\rho$ mit externem Resonator im empfangenden Lasersystem. $I_{1}=32.5 \mathrm{~mA}, T_{1}=19.4^{\circ} \mathrm{C}$, $T_{2}=19.2^{\circ} \mathrm{C}$.

berechneten Synchronisationsindex in Abbildung [5.6 überein. Synchronisation kann an den Punkten (d) und (f) angetroffen werden. In den Auftragungen der Punkte (c), (g) und (h) tritt der Effekt auf, dass zwar einige der Intensitätseinbrüche des empfangenden Lasers synchron mit dem treibenden Laser stattfinden, jedoch der empfangende Laser zusätzliche Intensitätseinbrüche - unabhängig vom treibenden Laser - vollführt.

\subsection{2 Übergang von Synchronisation zu Antisynchronisation}

Ein sehr interessanter Effekt ist in der Umgebung um Punkt (b) aus Abbildung [5.6]auszumachen, wie die Intensitätsauftragung in Abbildung [5.7b zeigt. In der Auftragung findet zeitgleich mit dem Intensitätseinbruch des treibenden Systems ein Intensitätsanstieg des empfangenden Systems statt. Erst nach einer gewissen Verzögerungszeit bricht auch die Intensität im empfangenden System ein. Der Synchronisationindex $S$ kann hier nicht richtig weiterhelfen, da je nach genauer Ausprägung des Intensitätssprungs bzw. des Intensitätseinbruches Synchronisation bzw. Antisynchronisation detektiert werden kann.

Diese Problematik kann sehr schön mit der Darstellung eines Ausschnitts der Kreuzkorrelationsfunktion $C_{X Y}(\tau)$ (ein kleiner Bereich um $\tau=0$ ) verdeutlicht werden. In 

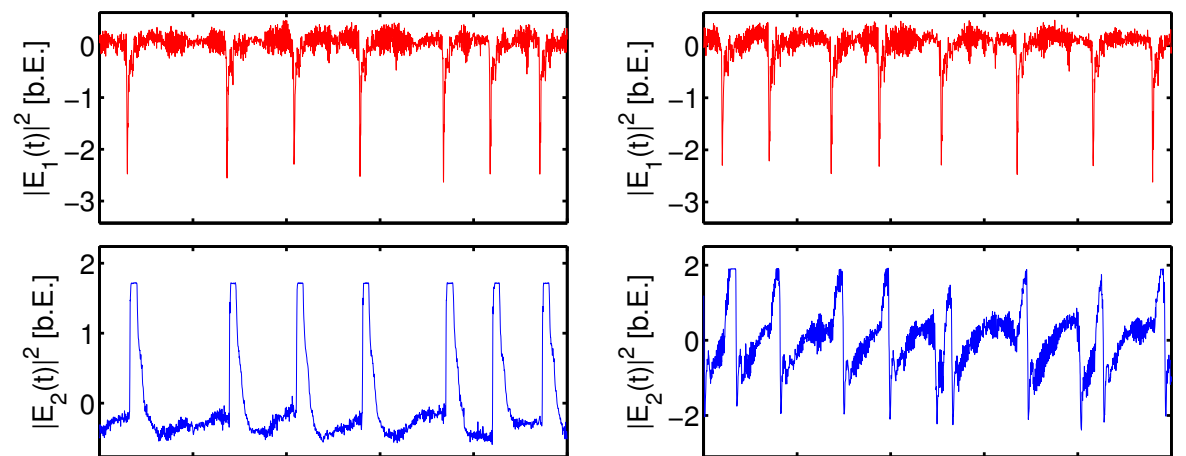

(a)
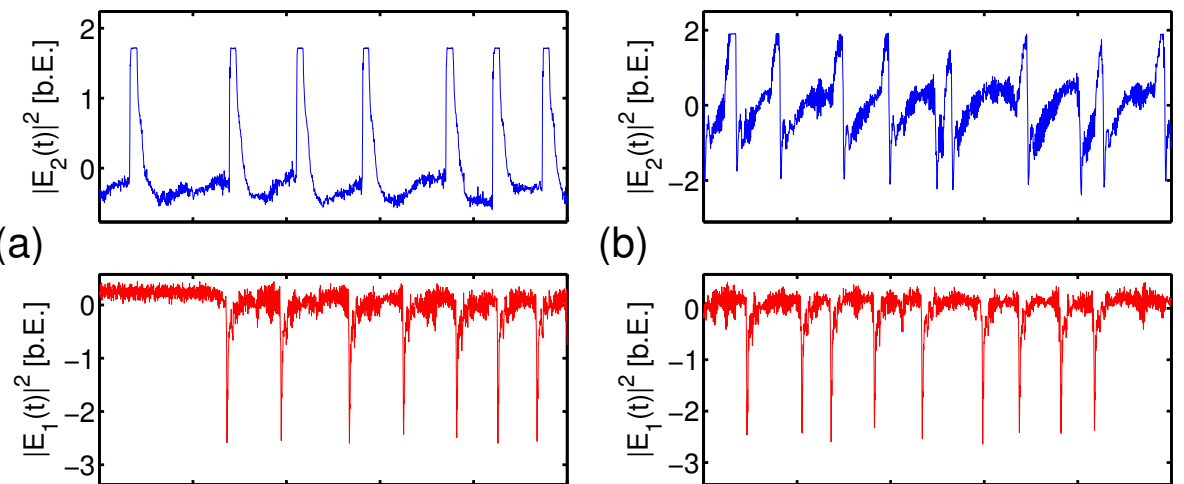

(b)

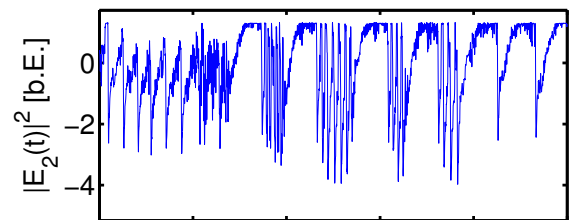

(c)
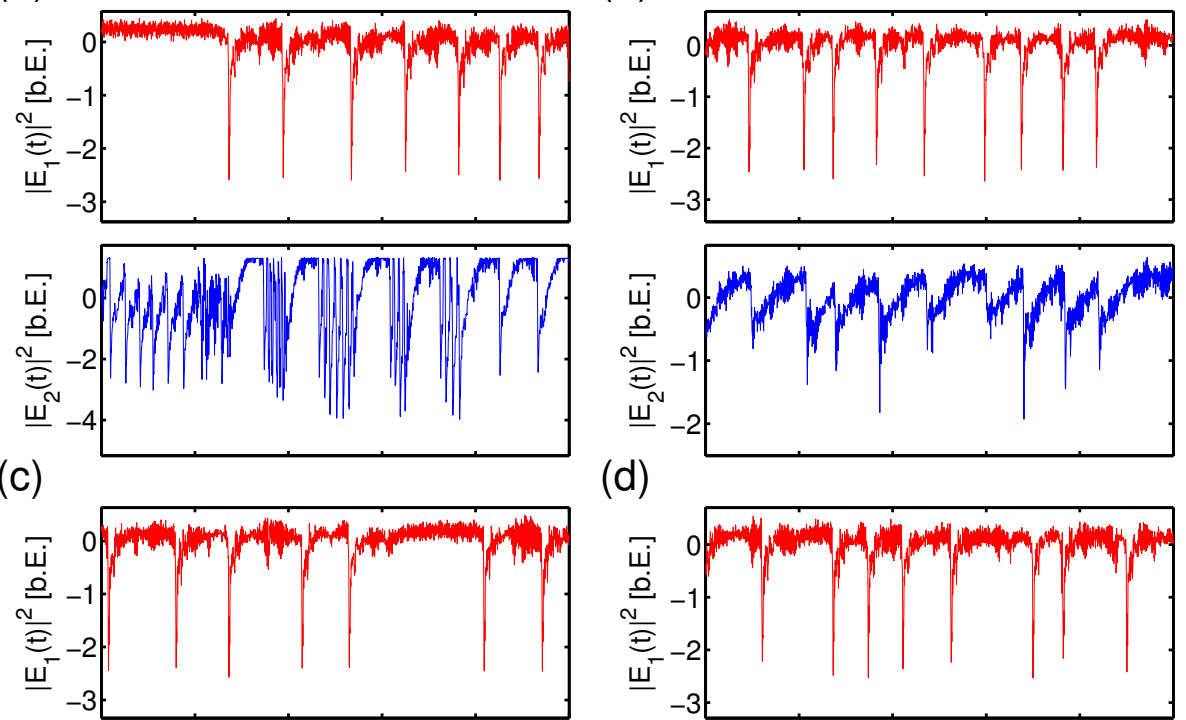

(d)

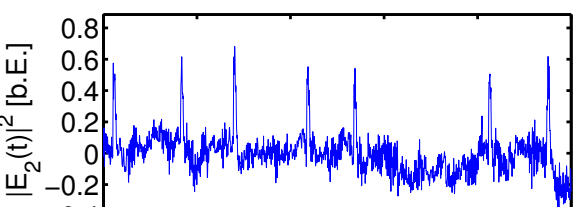

(e)
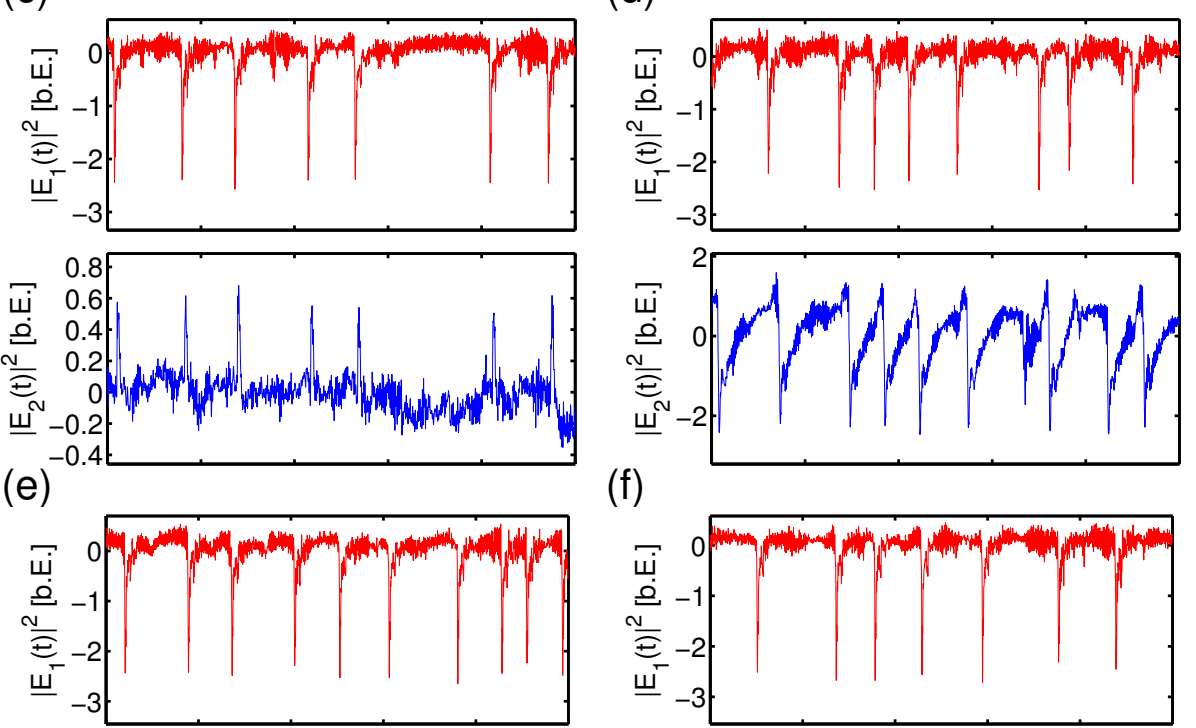

(f)
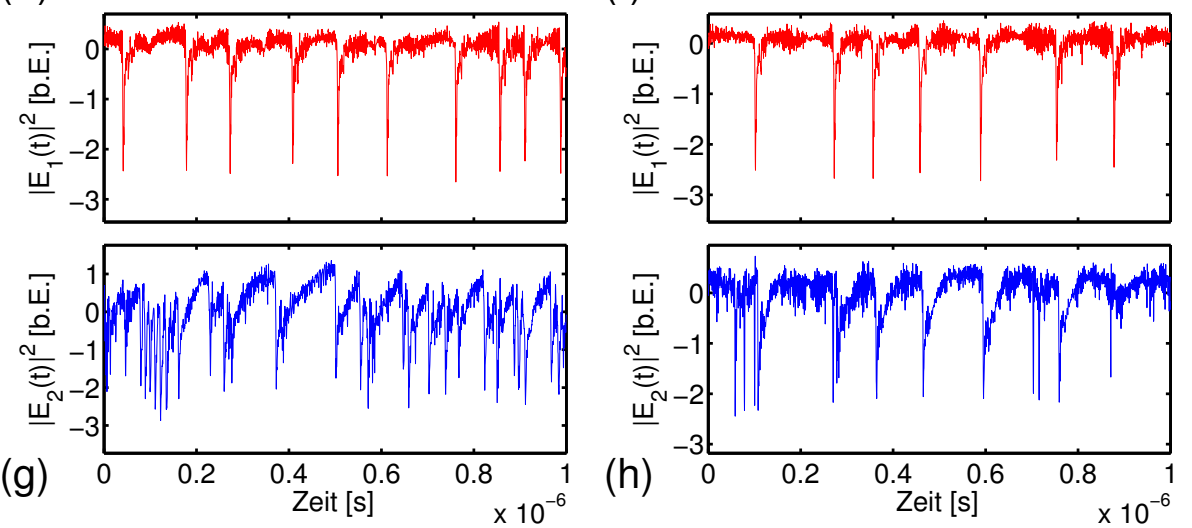

Abbildung 5.7: Zeitreihen der Laserintensität zu verschiedenen Parameterkombinationen aus Kopplungsstärke und Pumpstrom gemäß den Punkten (a, b, c, d, e, f, g und h) aus Abbildung [5.6. 


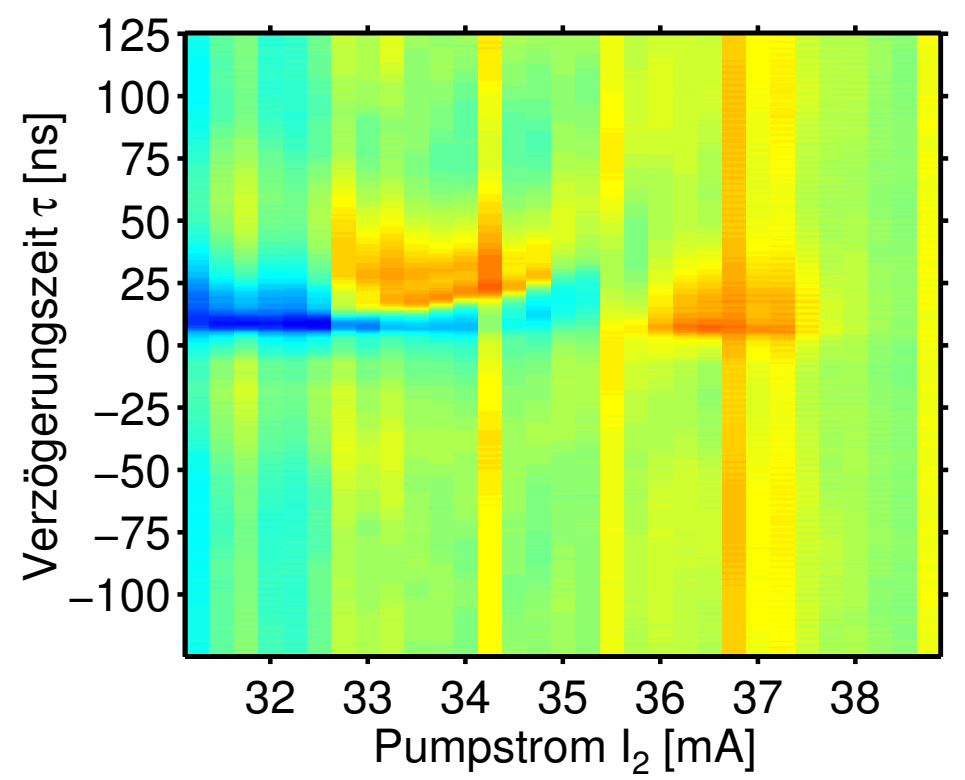

Abbildung 5.8: Ausschnitt der Kreuzkorrelationsfunktion $C_{X Y}(\tau)$ farbkodiert für verschiedene Pumpströme $I_{2}$ und Verzögerungszeiten $\tau$ aufgetragen (mit externem Resonator im empfangenden System).

Abbildung 5.8 habe ich $C_{X Y}(\tau)$ farbkodiert gegen $\tau$ und den Pumpstrom $I_{2}$ aufgetragen. Ganz links im Pumpstrombereich bis ca. $I_{2}=33 \mathrm{~mA}$ herrscht Antisynchronisation: es wird nur ein stark gefärbter blauer Bereich bei der Verzögerungszeit von ca. $\tau=5.5$ ns dargestellt. Die Unschärfe dieser blauen Linie zeigt eine gewisse Streuung der vorkommenden Verzögerungszeiten, die bei den vielen korrelierten Intensitätseinbrüchen auftreten. Ab dem Pumpstrom $I_{2}=33 \mathrm{~mA}$ wird zum einen diese blaue Linie sehr schmal, d.h. es gibt kaum noch Variation in der Verzögerungszeit, zum anderen kommt eine weitere rote Linie hinzu. Dies deutet an, dass auch eine positive Korrelation zu einer anderen Verzögerungszeit auftritt. Genau in diesem Bereich in dem sowohl eine hohe positive Korrelation als auch eine hohe negative Korrelation auftritt, ergeben sich Intensitätsverläufe, wie sie in Abbildung [5.7b zu erkennen sind. Interessant wird dieser Bereich durch die in Abbildung 5.8 gut zu erkennenden Anstieg der Verzögerungszeit $\tau$ für diese positive Korrelation bei Zunahme des Pumpstroms $I_{2}$. Ab einem Pumpstrom von ca. $I_{2}=34.2 \mathrm{~mA}$ verschwindet die Antikorrelation. Im Bereich von ca. $I_{2}=34.5 \ldots 36 \mathrm{~mA}$ ist überhaupt keine ausgeprägte Korrelation, weder positiv noch negativ, erkennbar. Erst ab ca. $I_{2}=36 \mathrm{~mA}$ zeigt sich wieder eine starke positive Korrelation bei derselben Verzögerungzeit wie anfangs die negative Korrelation.

Dieselbe Auftragung wie in Abbildung 5.8 habe ich auch für den Fall ohne externen Resonator erstellt (Abb. 5.9). Im selben Pumpstrombereich zeigt sich hier lediglich eine negative Korrelation. Die Verzögerungszeit scheint sich jedoch sehr ähnlich zu 


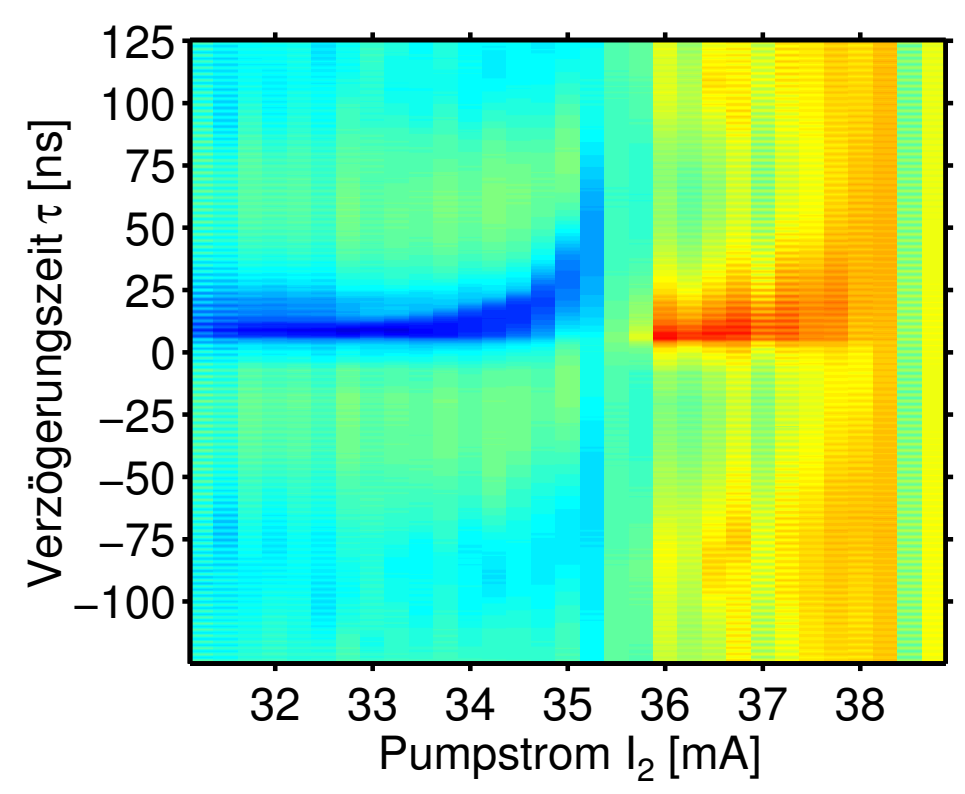

\begin{abstract}
Abbildung 5.9: Ausschnitt der Kreuzkorrelationfunktion $C_{X Y}(\tau)$ farbkodiert für verschiedene Pumpströme $I_{2}$ und Verzögerungszeiten $\tau$ aufgetragen (ohne externen Resonator im empfangenden System).
\end{abstract}

verändern wie die positive Korrelation in der vorangegangenen Auftragung. Um diesen Effekt der sich ändernden Verzögerungszeit genauer zu untersuchen, habe ich für diesen Parameterbereich $I_{2}=33.1 \ldots 35.5 \mathrm{~mA}$ mehrere Zeitreihen des Intensitätssignals beider Laser aufgenommen. Ausgehend von einem Intensitätseinbruch des treibenden Lasers (rote Kurve) ist in Abbildung [5.10 die Reaktion des Empfangslasers mit externem Resonator bzw. in Abbildung 5.11 ohne externen Resonator dargestellt.

In der Abbildung 5.10 ist für die Kurven (a) und (d) gut zu erkennen, dass der Empfangslaser mit einer Verzögerungszeit von ca. $\tau=5.5 \mathrm{~ns}$ auf das Signal des treibenden Lasers (rot) reagiert. Für die Kurven (b) und (c) gilt ebenfalls diese Verzögerungszeit, die Reaktion des empfangenden Laser jedoch nur schwach an einem leichten Knick der Intensitätskurve nach oben bemerkbar. Der eigentliche zu erwartenden Intensitätseinbruch verschiebt sich von (a) bis (c) zu immer höheren Verzögerungszeiten. Bei einer noch weitere Erhöhung des Pumpstroms $I_{2}$ reduziert sich diese Verzögerungszeit jedoch relativ abrupt wieder auf die eingangs erwähnten 5.5 ns. Hier ist jedoch noch zu betonen, dass die in Abbildung 5.10 dargestellten Signale beispielhaft zu sehen sind. Im Experiment ergibt sich eine relativ große Streuung der auftretenden Verzögerungszeiten, wie auch näherungsweise durch die Breite des Korrelationsbereiches in Abbildung 5.8 zu erkennen ist.

Das Verhalten ohne externen Resonator im Empfangssystem ist in gewisserweise ähn- 


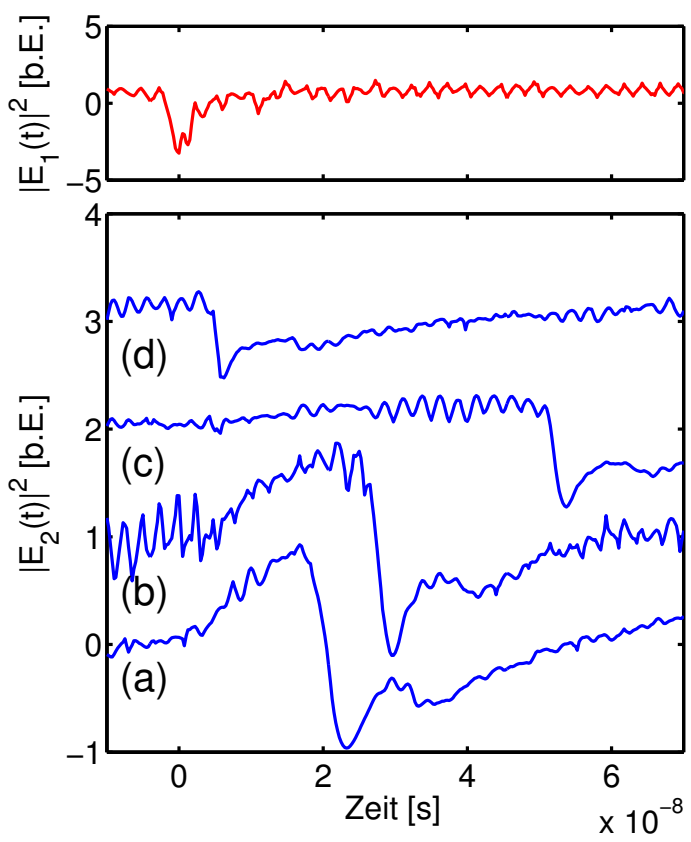

Abbildung 5.10: Zeitreihen der Laserintensität für den Parameterbereich der sich ändernden Verzögerungszeit für den Fall mit externem Resonator im Empfangssystem. Rot: treibender Laser, blau: Empfangslaser, (a) $I_{2}=33.8 \mathrm{~mA}$, (b) $I_{2}=34.3 \mathrm{~mA}$, (c) $I_{2}=34.7 \mathrm{~mA}$, (d) $I_{2}=35.2 \mathrm{~mA}$. Die Intensitätskurven b, c und d wurden zur besseren Darstellung vertikal um 1, 2, 3 bzw. 4 Einheiten nach oben verschoben. 


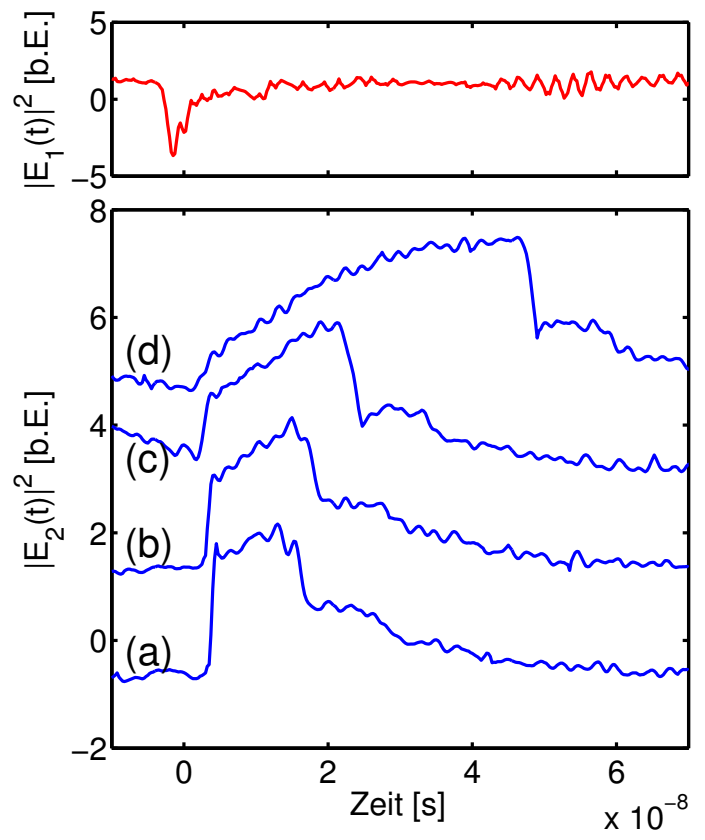

Abbildung 5.11: Zeitreihen der Laserintensität für den Parameterbereich der sich ändernden Verzögerungszeit für den Fall ohne externem Resonator im Empfangssystem. Rot: treibender Laser, blau: Empfangslaser, (a) $I_{2}=$ $33.8 \mathrm{~mA}$, (b) $I_{2}=34.3 \mathrm{~mA}$, (c) $I_{2}=34.7 \mathrm{~mA}$, (d) $I_{2}=35.2 \mathrm{~mA}$. Die Intensitätskurven b, c und d wurden zur besseren Darstellung vertikal um 2, 4 bzw. 6 Einheiten nach oben verschoben. 
lich mit dem zuvor genannten. Auch hier lässt sich sehr gut an den Intensitätskurven von (a) bis (d) in Abbildung 5.11 eine Reaktionsverzögerung des Empfangssystems von ca. $\tau=5.5 \mathrm{~ns}$ ausmachen. Der Laser reagiert ebenfalls mit einem Intensitätsanstieg der jedoch deutlich höher ausfällt als im vorherigen Fall (die Intensitätskurven der Auftragungen in Abbildung [5.10 und 5.11 besitzen die gleiche Skalierung). Mit einer je nach Pumpstrom $I_{2}$ variablen Verzögerungszeit ergibt sich auch in diesen Intensitätskurven ein Einbruch, der jedoch relativ gesehen zum Intensitätsanstieg gegenüber den Messung in Abbildung [5.10 geringer ausfällt. Die Tendenz der Zunahme der Verzögerungszeiten stimmen für die Kurven (a) bis (c) für beide Abbildungen 5.10 und 5.11 überein, jedoch nicht die genauen Verzögerungszeiten. Dies hängt jedoch mit hoher Wahrscheinlichkeit mit bereits erwähnten Streuung dieser Verzögerungszeiten zusammen. Einen substantiellen Unterschied zeigt jedoch für den Fall (d). Die Verzögerungszeit für den Intensitätseinbruch für die Kurve 5.11 d steigt weiter bis der Einbruch genau den Reaktionspunkt des empfangenden Laser auf den nächsten Intensitätseinbruch des treibenden Lasers erreicht. Ab diesem Punkt ist der Empfangslaser (identisch) synchronisiert mit dem treibenden Laser. Dieser Vorgang lässt sich in Abbildung $\mathbf{5 . 1 2}$ sehr gut erkennen. Die Intensitätseinbrüche mit anschließend relativ kurzen Aufschaukelphasen sind bereits vollständig synchronisiert. Für längere Aufschaukelphasen bricht die Intensität des Empfangslasers aber bereits viel früher als die des treibenden Lasers zusammen.

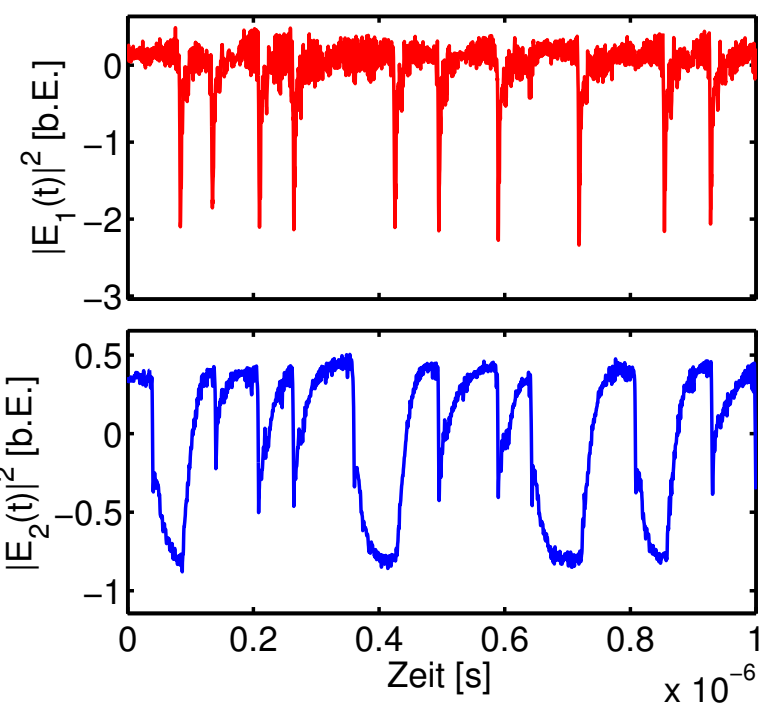

Abbildung 5.12: Übergang von der Antisynchronisation zur Synchronisation ohne externen Resonator im Empfangssystem. $I_{2}=35.5 \mathrm{~mA}$. 


\title{
5.3.3 Parameterbereiche der Synchronisation und Antisynchronisa- tion
}

Von entscheidender Bedeutung für die Erreichung der Synchronisation ist die Übereinstimmung der Wellenlänge beider Laser, welche im wesentlichen über die Temperatur und den Pumpstrom variiert werden kann. In Abbildung 5.13 habe ich den Synchronisationsindex farbkodiert über der Temperatur und dem Pumpstrom des Empfangslasers aufgetragen. Für diesen Fall ohne externen Resonator sind sehr schön die Bereiche der Synchronisation (rot) und die Bereiche der Antisynchronisation (blau) zu erkennen. In den hellgrün dargestellten Zwischenräumen ist entweder nur minimale Korrelation oder sehr geringe Fluktuation des Empfangslasers zu verzeichnen, in diesen Bereichen sind beide Laser nicht synchronisiert (Farbtabelle in Abb. C.4).

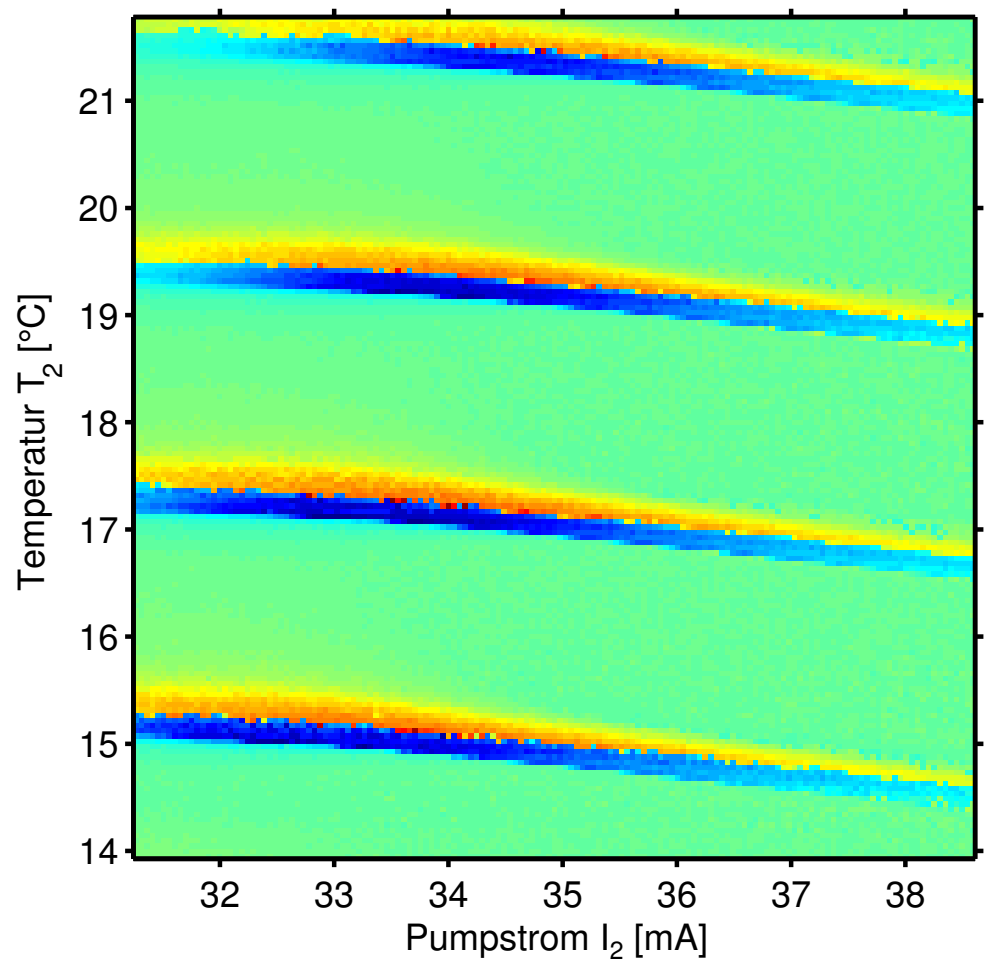

\begin{abstract}
Abbildung 5.13: Auftragung des Synchronisationsindexes für verschiedene Temperaturen und Pumpströme des Empfangslasers bei konstant gehaltenen Parametern des treibenden Lasers $I_{1}=32.5 \mathrm{~mA}, T_{1}=19.4^{\circ} \mathrm{C}$, kein externer Resonator im Empfangssystem.
\end{abstract}

Die Gebiete der Synchronisation liegen in direkter Nachbarschaft zu Gebieten der Antisynchronisation, wobei der Antisynchronisationsbereich in dieser Darstellung immer „unterhalb“ des angrenzenden Synchronisationsbereiches liegt. Desweiteren wiederholen sich diese bandartigen Strukturen mit konstantem Temperaturabstand. In 
Abbildung 5.14 ist ebenfalls der Synchronisationsindex farbkodiert dargestellt, in diesem Fall wurden die Temperatur und der Pumpstrom des treibenden Lasers variiert. Die Parameter des Empfangslasers wurden hierbei konstant gehalten. Die Auftragung zeigt ein sehr ähnliches Bild wie Abbildung [5.13, auch hier wiederholen sich die bandartigen Strukturen mit demselben Temperaturabstand, die Synchronisationsabfolge ist hier hingegen vertauscht. Der Antisynchronisationsbereich (blau) liegt hier immer oberhalb des direkt angrenzenden Synchronisationsbereiches.

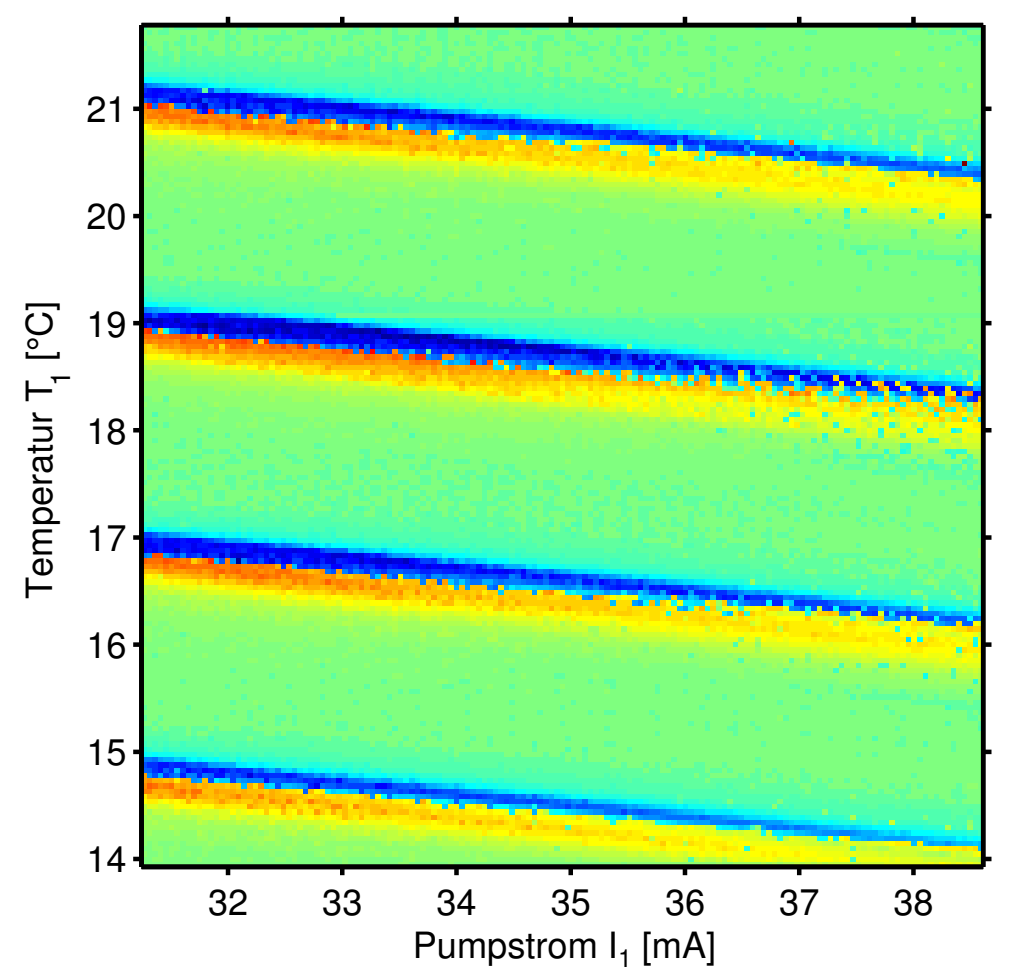

\begin{abstract}
Abbildung 5.14: Auftragung des Synchronisationsindexes für verschiedene Temperaturen und Pumpströme des treibenden Lasers bei konstant gehaltenen Parametern des Empfangslasers $I_{2}=32.5 \mathrm{~mA}, T_{2}=19.4^{\circ} \mathrm{C}$, kein externer Resonator im Empfangssystem.
\end{abstract}

Der Grund für die Umkehrung der Synchronisationsreihenfolge liegt in der Wellenlängendifferenz beider Laser. Im Allgemeinen kann angenommen werden, dass für der Erreichung der Synchronisation die Wellenlängen beider Laser identisch sein müssen, d.h.

$$
\lambda_{1}-\lambda_{2} \approx 0
$$

Ist hingegen die Wellenlänge des empfangenden Lasers $\lambda_{2}$ kleiner als die des treibenden $\lambda_{1}$, so entsteht Antisynchronisation:

$$
\lambda_{1}-\lambda_{2} \gg 0
$$


Dies kann entweder durch Verringerung von $\lambda_{1}$, also einer Verringerung von $I_{2}$ oder $T_{2}$, oder durch Erhöhung von $\lambda_{1}$ und demnach einer Erhöhung von $I_{1}$ oder $T_{1}$, geschehen. Diese Hypothese wird in Kapitel [ ] durch die numerischen Simulationen bestätigt.

\subsubsection{Messung des optischen Leistungspektrums}

Die Hypothese aus dem letzten Abschnitt erklärt jedoch nicht das Zustandekommen der sich wiederholenden Bänder aus Synchronisation und Antisynchronisation. Möglicherweise hängt dieser Effekt mit der Modenstruktur der Laser zusammen. Um dies zu klären, habe ich das Leistungsspektrum des Lichtes mit einem Spektrometer für verschiedene Temperaturen beider Laser aufgenommen. In dem verwendeten Spektrometer fällt das eingestrahlte Licht auf ein Gitter und wird auf eine CCD-Zeile abgebildet, die durch eine eingebaute Elektronik sequenziell mit einem Takt von ca. $30 \mathrm{~Hz}$ ausgelesen wird. In Abbildung [5.15] ist der Aufbau für diese Messung dargestellt. Da nur ein Spektrometer zur Verfügung stand, konnte auch nur ein Laser zur selben Zeit gemessen werden. Es wurde also erst der treibende Laser für verschiedene Temperaturen $T_{1}$ mit dem Spektrometer untersucht (Abb. [5.16), in einem zweiten Schritt wurde der empfangende Laser für dieselben Parameter $T_{1}$ vermessen (Abb. 5.17). Für diese hochaufgelöste Darstellung wurde die in Abb. C.2 gezeigte Farbtabelle verwendet.

Es ist klar erkennbar, dass der treibende Laser je nach Temperatur auf ca. 3-5 verschiedenen Wellenlängen emittiert. Hierbei handelt es sich um die im Kapitel 3 bereits erwähnten longitudinalen Moden, die für diese Laserart typisch sind. Inwieweit alle diese Moden gleichzeitig emittieren oder eine Dynamik von Mode zu Mode in schneller Folge auftritt kann in diesem experimentellen Aufbau nicht geklärt werden, da hier nur eine Mittelung über eine Belichtungszeit der CCD-Zeile gemacht wird.

Ein weiterer wichtiger Punkt ist, dass sich die Wellenlängen der Moden durch Veränderung der Temperatur nicht (oder nicht erkennbar) stetig, sondern sprunghaft ändern. Dies ist in der Tat ungewöhnlich und entspricht nicht dem erwarteten Verhalten für Laserdioden, welches ich in Abschnitt 2.6 beschrieben habe. Genauere Untersuchungen dieses Phänomens brachten keinen Aufschluss über die Ursache. Der externe Resonator scheidet als Ursache aus, da der Effekt auch ohne diesen auftritt. Ein Messartefakt ist ebenfalls ausgeschlossen, da mit dem selben Aufbau das Spektrum eines anderen Lasertyps korrekt wiedergeben werden konnte.

In der Auftragung 5.17 lässt sich sehr schön die spektrale Reaktion des empfangenden Lasers auf das Signal des treibenden Lasers erkennen. Bei den meisten Temperaturen $T_{1}$ tritt keine Beeinflussung des empfangenden Lasers auf, er emittiert praktisch 


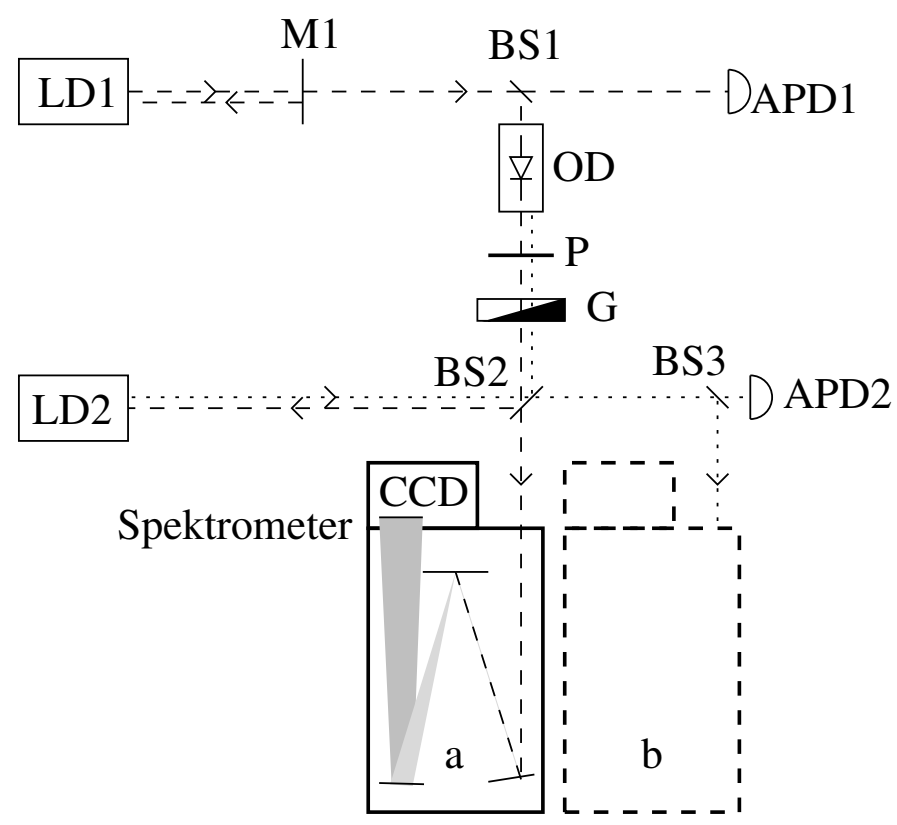

\begin{abstract}
Abbildung 5.15: Aufbau zur Messung des optischen Leistungsspektrums beider Laserdioden für verschiedene Temperaturen $T_{1}, T_{2}$. Spektrometer in Position (a) zur Aufnahme der treibenden Laserdiode und Position (b) zur Aufnahme der empfangenden Laserdiode.
\end{abstract}

ausschließlich auf einer einzigen Mode 3 . Im Bereich von $T_{1} \approx 16^{\circ} \mathrm{C}, T_{1} \approx 18.5^{\circ} \mathrm{C}$, $T_{1} \approx 21^{\circ} \mathrm{C}$ und $T_{1} \approx 23.5^{\circ} \mathrm{C}$ synchronisiert das Spektrum des empfangende Laser mit dem treibenden. Innerhalb des kleinen Temperaturintervall dieser Synchronisationsgebiete führt der empfangende Laser sogar die Modensprünge des treibenden Lasers synchron aus.

Eine genauere Analyse dieser Spektrogramme zeigt relativ deutlich den Mechanismus der Wiederholung der Synchronisationsgebiete. Hierzu habe ich in Abbildung 5.18 die Spektrogramme aus den Abbildungen 5.16] und 5.17 überlagert. Rot wird das Spektrum des treibenden Lasers dargestellt, blau das des empfangenden Lasers. Identischen Wellenlängen in beiden Lasern werden grün dargestellt. Werden die Modenfrequenzen des treibenden Lasers auf die anhand der schwarzen Linien skizzierten Weise verbunden, zeigt sich, dass für jeden Synchronisationsbereich eine dieser Linien die Referenzwellenlänge $\lambda_{0}$ und demnach die Wellenlänge des Empfangslasers schneidet.

Um zu untersuchen, inwieweit sich der Umschaltvorgang von Synchronisation zu Antisynchronisation auch auf das optische Leistungsspektrum auswirkt, ist es sinnvoll einen einzigen Synchronisationsbereich hochaufgelöst dem Synchronisationsindex ge-

\footnotetext{
${ }^{3}$ Die Wellenlänge dieser Mode wird in der Abbildung [5.16 und 5.17 als Referenzwellenlänge $\lambda_{0}$ verwendet, da das Spektrometer keine sinnvolle Eichung der Wellenlänge besitzt.
} 


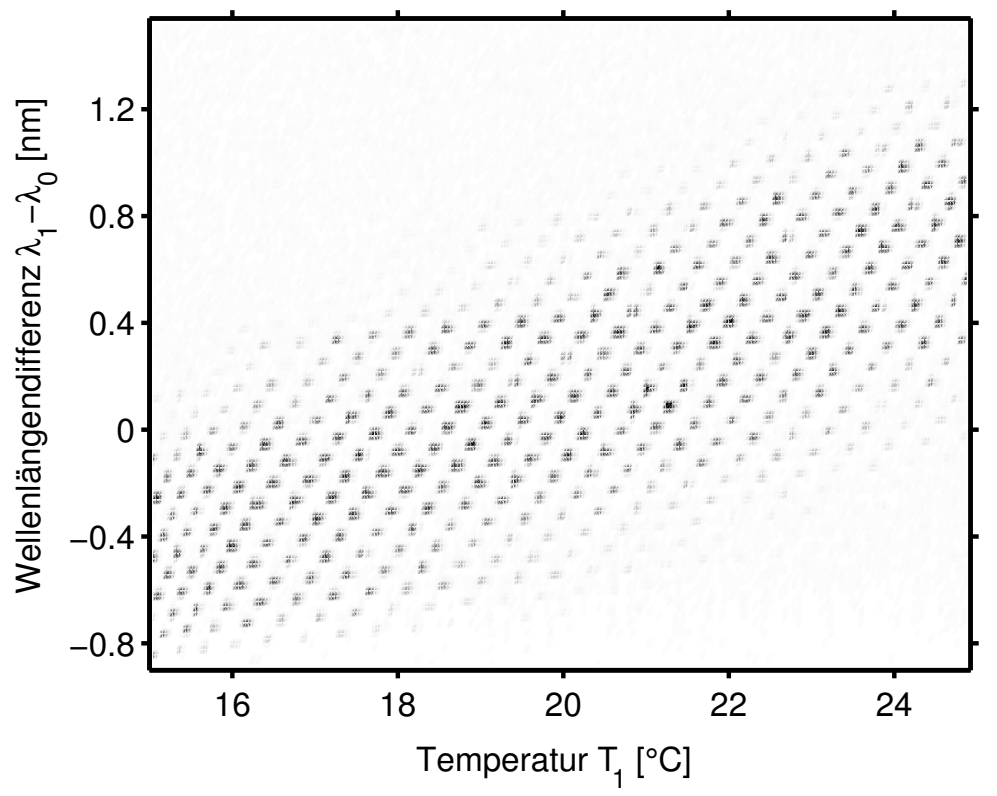

Abbildung 5.16: Optisches Leistungsspektrum des treibenden Lasers für verschiedene Temperaturen des treibenden Lasers $T_{1}$ aufgetragen.

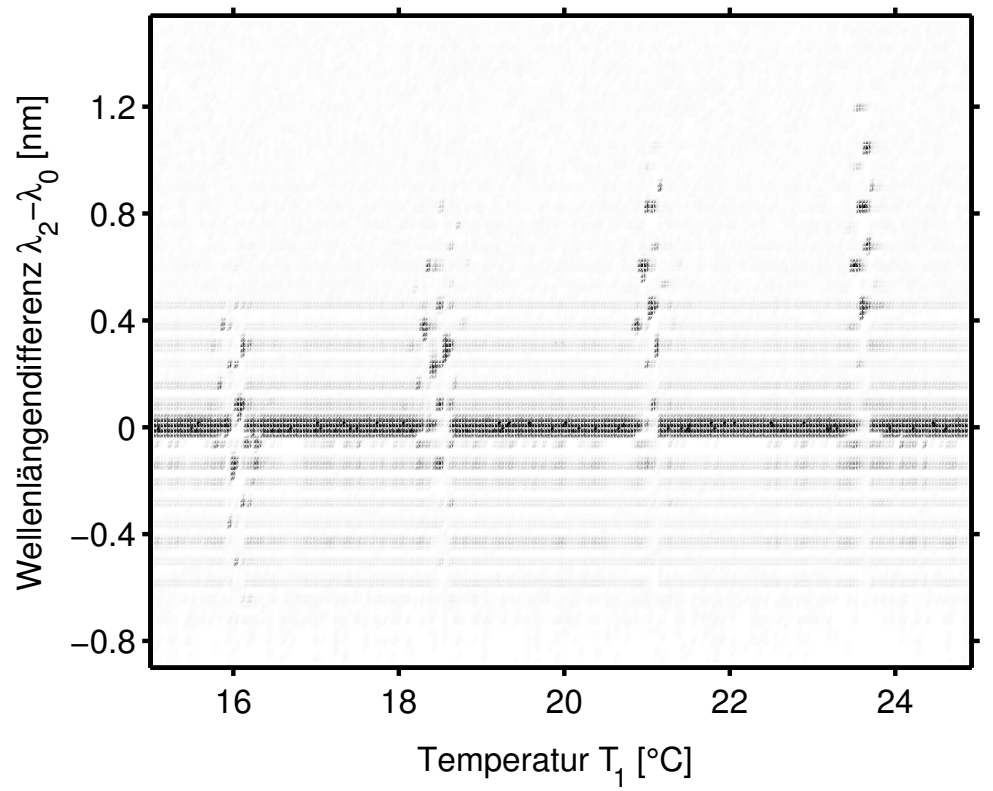

Abbildung 5.17: Optisches Leistungsspektrum des empfangenden Lasers für verschiedene Temperaturen des treibenden Lasers $T_{1}$ aufgetragen. 


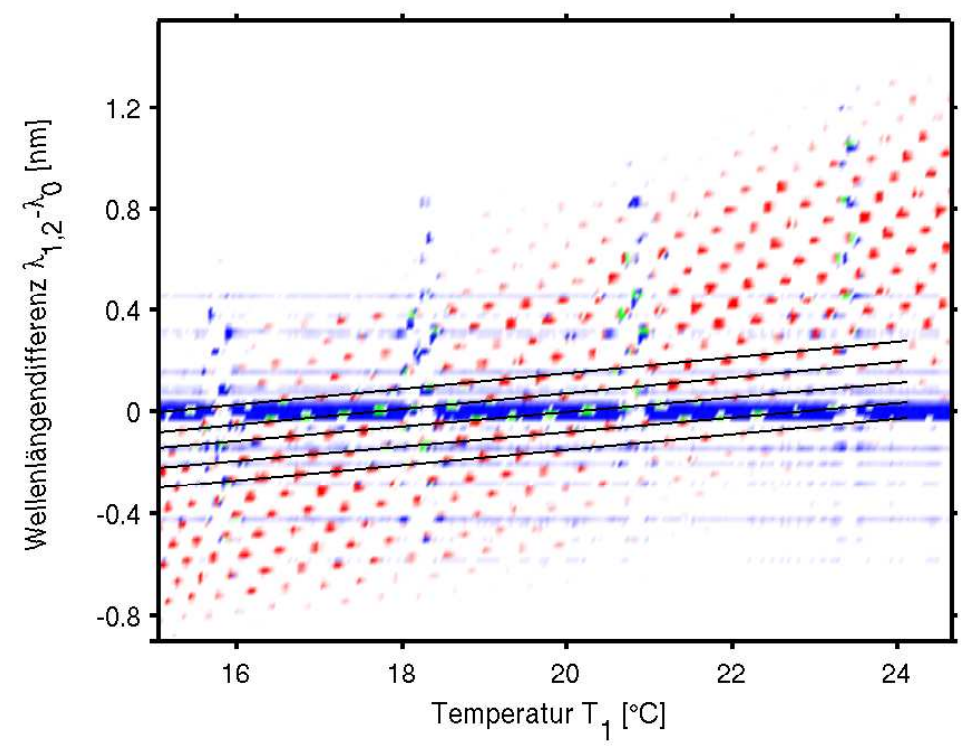

Abbildung 5.18: Überlagerung der Spektrogramme aus den Abbildungen 5.16 und 5.17

genüberzustellen. Für die im folgenden vorgestellten Messungen wurde derselbe Messaufbau wie zuvor verwendet (Abb. 5.15). Zusätzlich wurden noch die Intensitätssignale beider Laser aufgenommen und der Synchronisationsindex berechnet. Da jedoch die einzelnen Moden von Messung zu Messung sehr starken Intensitätsschwankungen unterworfen sind, wurde jedes Spektrum durch Mittelung über mehrere Messungen gewonnen.

In Abbildung [5.19a ist das optische Leistungsspektrums des treibenden Lasers über der Temperatur $T_{1}$ farbkodiert aufgetragen (Farbtabelle Abb. C.3). In einem Balken oberhalb dieses Spektrogramms ist die farbkodierte Auftragung des Synchronisationsindex der Gesamtintensitäten für die jeweilige Temperatur $T_{1}$ mit der bisher verwendeten Farbgebung dargestellt (Farbtabelle Abb. C.4).

Abbildung [5.19b zeigt das optische Leistungsspektrum des Empfangslasers korrespondierend mit der Auftragung in Abbildung 5.17. Im Bereiche der Synchronisation im Temperaturbereich von $T_{1} \approx 18.75-18.9^{\circ} \mathrm{C}$ (dunkelrote Farbkodierung auf dem Balken oberhalb der Auftragungen), treten im Empfangslaser dieselben Modenfrequenzen wie im treibenden Laser auf. Auch der Modenfrequenzwechsel wird von beiden Lasers synchron ausgeführt. In der Übergangsphase zwischen Synchronisation und Antisynchronisation beider Laser bei $T_{1} \approx 18.9-19.1^{\circ} \mathrm{C}$ vollführt der treibende Laser einen weiteren Modenfrequenzwechsel. Der Empfangslaser zeigt diesen Wechsel ebenfalls, jedoch mit einer stark reduzierten Intensität. Weitere Frequenzwechsel des treiben- 


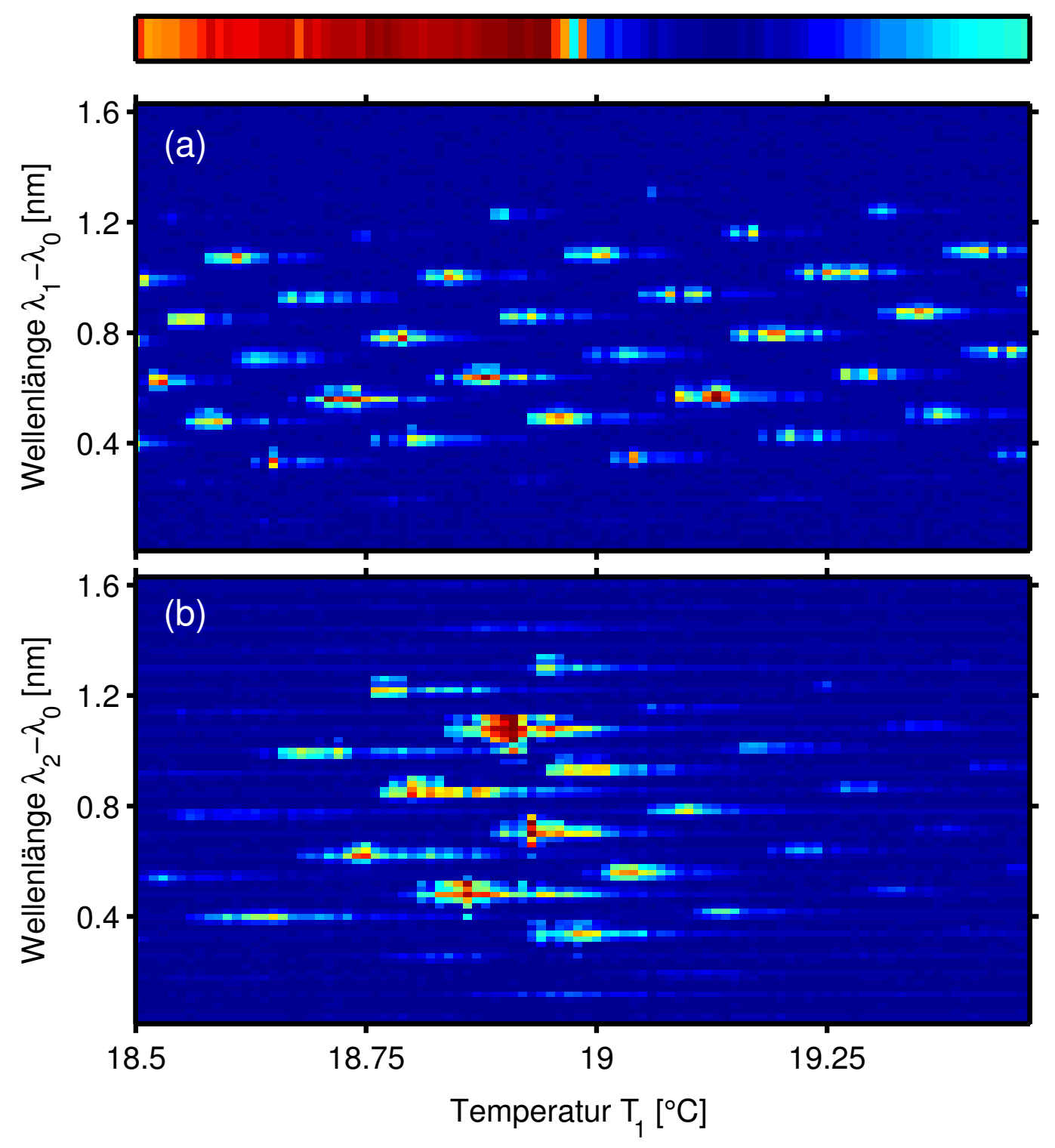

Abbildung 5.19: Auftragung des optischen Leistungsspektrums (a) des treibenden Lasers und (b) des empfangenden Lasers farbkodiert über der Temperatur $T_{1}$. Der Balken oberhalb der Spektrogramme zeigt farbkodiert den Synchronisationsindex zu der jeweiligen Temperatur (rot: Synchronisation, blau: Antisynchronisation, gelb/grün: keine Korrelation oder keine Intensitätsfluktuation des Empfangslasers). 


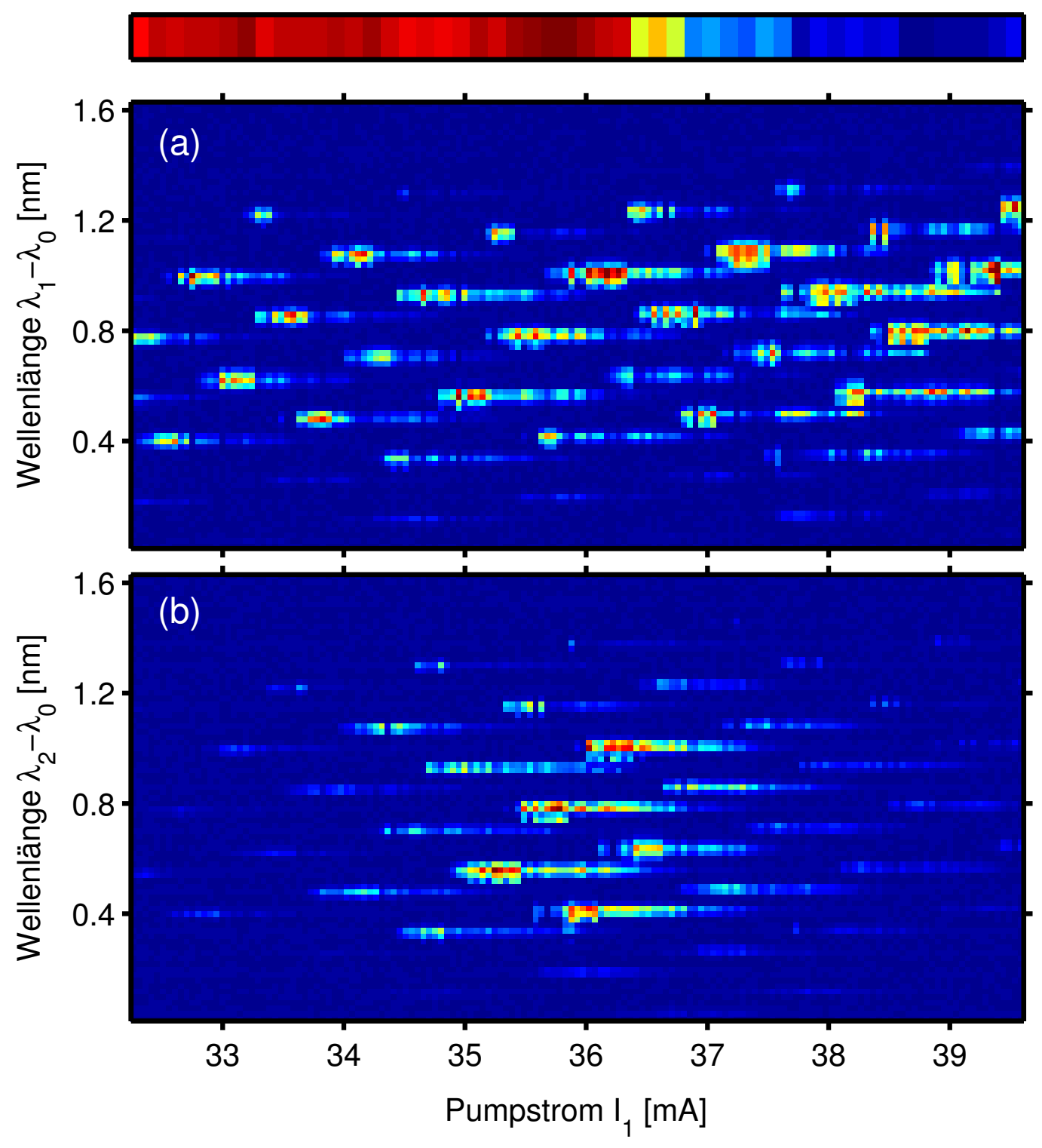

Abbildung 5.20: Auftragung des optischen Leistungsspektrums (a) des treibenden Lasers und (b) des empfangenden Lasers farbkodiert über dem Pumpstrom $I_{1}$. Der Balken oberhalb der Spektrogramme zeigt farbkodiert den Synchronisationsindex (rot: Synchronisation, blau: Antisynchronisation, gelb/grün: keine Korrelation oder keine Intensitätsfluktuation des Empfangslasers). 
den Lasers sind synchron ebenfalls im Empfangslaser erkennbar, jedoch mit praktisch verschwindender Intensität.

Abbildung [5.20] zeigt dieselbe Auftragung wie Abbildung [5.19, jedoch mit dem Pumpstrom $I_{1}$ als Parameter. Der Laser zeigt ein nahezu identisches Verhalten wie bei Temperaturänderung.

\subsubsection{Modensynchronisation}

Bisher habe ich zur Bestimmung der Synchronisation lediglich die Gesamtintensität betrachtet. Bei der Aufnahme der Spektren für die Auftragungen im letzten Abschnitt musste aufgrund der starken Fluktuationen über mehrere Messungen gemittelt werden, um eine aussagekräftige Darstellung aller aktiven Moden zu bekommen. In diesem Abschnitt sollen diese Modenfluktuation in hoher zeitlicher Auflösung auf Synchronisation zwischen beiden Lasern untersucht werden (das Spektrometer zeigt diese Fluktuationen nur mit einer Wiederholfrequenz von $30 \mathrm{~Hz})$.

$\mathrm{Zu}$ diesem Zweck habe ich den in Abbildung 5.21 gezeigten Versuchsaufbau verwendet, in dem ein Monochromator zur Wellenlängenfilterung eingesetzt wird. Da dieses Instrument einen ca. $5 \mathrm{~mm}$ hohen Schlitz einstellbarer Breite spektral filtert und abbildet ist es möglich beide Laser gleichzeitig mit demselben Gerät zu filtern. Nachteil ist jedoch, dass die Beobachtung der Intensität auf dieselbe Wellenlänge für beide Laser begrenzt ist. Zur Beobachtung der direkten Synchronisation von Einzelmoden ist dies aber keine Einschränkung, da angenommen werden kann, dass nur das elektrische Feld von Moden mit näherungsweiser gleicher Wellenlänge sich gegenseitig beeinflussen kann.

Der Aufbau ist so gewählt, um möglichst viele Informationen auf einmal messen zu können. APD1 und APD2 messen die Gesamtintensität beider Laser, im Messrechner wird aus beiden Intensitätssignalen der Synchronisationsindex berechnet und abgespeichert. Das Licht des treibenden Lasers und des Empfangslasers werden mit Hilfe der beiden Strahlteiler BS1 und BS4 übereinander (in der Abbildung sind die Strahlen nebeneinander gezeichnet) mit einem vertikalen Abstand von ca. 3mm auf den $5 \mathrm{~mm}$ hohen Eingangschlitz des Monochromator geleitet. BS2 und BS3 dienen zur Vermittlung des Kopplungslichts vom treibenden Laser über den Faradayisolator (OD) zum Empfangslaser. Hinter dem Ausgangsschlitz des Monochromators werden die optisch gefilterten Intensitätsignale beider Laser mit den Detektoren APD3 und APD4 gemessen.

Im Gegensatz zum vorher verwendeten Spektrometer, ist es mit diesem Aufbau nicht möglich die absolute Intensität weder des ungefilterten noch des gefilterten Lichtes der 


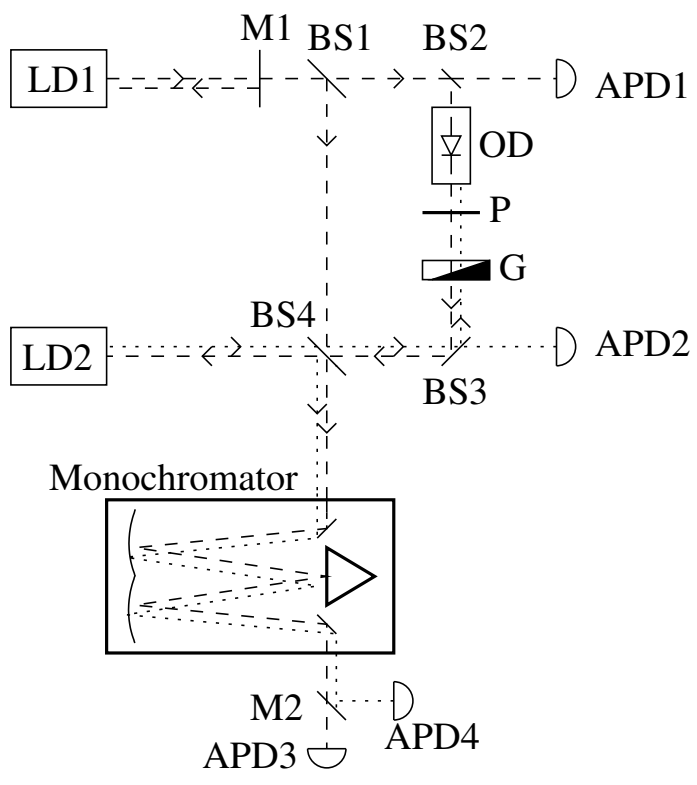

Abbildung 5.21: Aufbau zur Messung der Modenintensität beider Laser.

Laser zu messen. Grund ist der elektrische Aufbau der Detektoren (AC-Kopplung). Um jedoch trotzdem eine vergleichbare Größe ermitteln zu können, habe ich die SpitzeSpitze-Amplitude der Signale APD3 und APD4 verwendet. Bei konstanten Lichtsignalen verschwindet dieser Wert, sie können demnach hier nicht detektiert werden. Als vierten Wert wird der Synchronisationsindex der Signale von APD3 und APD4 berechnet und gespeichert.

Die spektrale Auflösung des Monochromators ist geringer als die des vorher verwendeten Spektrometers, jedoch bietet er an dem digitalen Steuergerät eine direkte Ablesung der gefilterten Wellenlänge.

Um eine vergleichbare Darstellung wie in den Abbildungen 5.19 bzw. 5.16 und 5.17 $\mathrm{zu}$ erreichen wurde in Abbildung $\mathbf{5 . 2 2}$ für die eben beschriebenen Größen aus (a) Spitze-Spitze-Amplitude des treibenden Lasers, (b) Spitze-Spitze-Amplitude des Empfangslasers sowie dem (c) Synchronisationsindex zwischen APD3- und APD4-Signalen für verschiedene Wellenlängen $\lambda$ des Monochromators und Temperaturen $T_{1}$ des treibenden Lasers aufgenommen und farbkodiert aufgetragen. Darüberhinaus wurde der bereits in den Abbildungen [5.19] und 5.20 verwendete Balken zur Darstellung des Synchronisationsindex zwischen APD1 und APD2 (also der Synchronisationindex der Gesamtintensitäten) oberhalb der Auftragungen dargestellt. Es werden die in Anhang C] beschriebenen Farbtabellen verwendet.

Die Auftragungen 5.22 and $5.22 \mathrm{~b}$ entsprechen im Prinzip den Abbildungen [5.16] und 5.17. Der Messaufwand ist jedoch hier erheblich höher, da jede Parameterkombination 


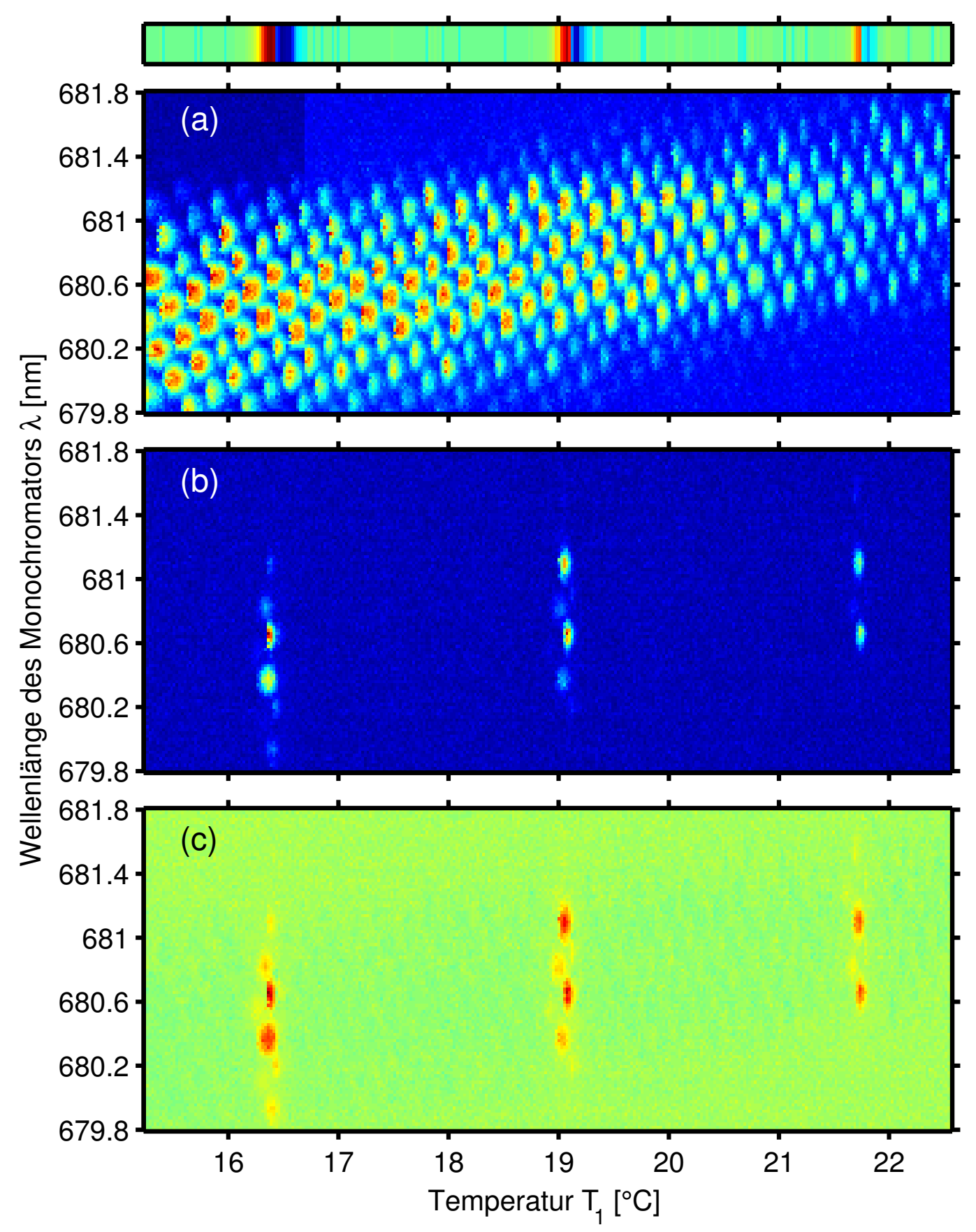

Abbildung 5.22: Auftragung zur Untersuchung auf Modensynchronisation. (a) Fluktuationsstärke des treibenden Lasers, (b) Fluktuationstärke des empfangenden Lasers, (c) Synchronisationsindex der gefilterten Laserintensitäten. Der Balken oberhalb der Auftragungen zeigt den Synchronisationsindex der Gesamtintensitäten. 
$\lambda, T_{1}$ elektromechanisch 4 angesteuert werden musste. In Auftragung [5.22 ist eindeutig zu erkennen, dass die im Empfangslaser innerhalb eines Synchronisationsgebietes fluktuierenden Moden (erkennbar in Auftragung [5.22 b) auch mit den Moden des treibenden Lasers synchronisiert sind: der Synchronisationsindex ist an den Positionen dieser Moden positiv. Offensichtlich existiert hier nur Synchronisation, keine Antisynchronisation. Überraschend ist auf den ersten Blick das Fehlen jeglicher Synchronisation im Bereiche der Antisynchronisation der Gesamtintensität der Laser. Dies lässt sich jedoch relativ leicht erklären: In Auftragung 5.22b ist im Antisynchronisationgebiet keine fluktuierende Mode zu erkennen. Bereits Abbildung [5.19] zeigt eine sehr geringe Intensität des Empfangslasers im Bereiche der Antisynchronisation. Dass die Empfindlichkeit des Detektionssystems aus Monochromator und APD geringer ist als die des Spektrometers ist schnell einzusehen; die Belichtungszeiten beider Systeme unterscheiden sich um einen Faktor $\approx 10^{7}$. Dieser extreme Unterschied kann durch die starke Verstärkung der APD und den größeren Lichteinfall aufgrund der niedrigeren Wellenlängenauflösung nicht komplett kompensiert werden.

Die Intensität der Moden des Empfangslasers liegt im Antisynchronisationsbereich also leider unterhalb der Detektionsschwelle. Beispielhaft habe ich die Intensitätssignale aller vier Detektoren bei der Parametereinstellung $\lambda=680.65 \mathrm{~nm}, T_{1}=16.37^{\circ} \mathrm{C} \mathrm{im}$ Parameterbereich der Modensynchronisation in Abbildung [5.23 aufgetragen. Die Gesamtintensität beider Laser, aufgenommen durch die Detektoren APD1 (treibender) und APD2 (empfangender) zeigen gut die Synchronisation der Gesamtintensität. Das optisch gefilterte Signal von APD3 für den treibenden Laser und APD4 für den empfangen Laser unterscheidet sich sehr deutlich von der Gesamtintensität beider Laser. Nichtsdestotrotz zeigen diese beiden Intensitätskurven eine gute Übereinstimmung. Da alle vier Intensitätskurven simultan aufgezeichnet worden sind, ist sehr gut zu erkennen, dass die beobachtete Mode zwar Intensitätseinbrüche aufweist, diese jedoch nicht immer synchron mit den Intensitätseinbrüchen der Gesamtintensität auftreten. Einige Intensitätseinbrüche fehlen komplett (z.B. im Bereich von $t=0.1-0.4 \mu \mathrm{s}$ ) oder es sind zusätzliche vorhanden, die sich nicht auf die Gesamtintensität auswirken.

Dies legt nahe, dass die Dynamik zwischen den Moden komplizierter sein muss, als von den einfacheren Multimoden-Modellen angenommen wurde. Die Dynamik zwischen den Moden kann auch nicht näherungsweise synchron verlaufen, dann wäre die Modenintensität einer Einzelmode gleich der Gesamtintensität des Lasers. Da die oben beobachtete Mode beispielsweise einige Intensitätseinbrüche der Gesamtinten-

\footnotetext{
${ }^{4}$ Die Wellenlänge des Monochromators wird durch das Verdrehen eines Spiegelgitters durch einen Schrittmotor erreicht. Die Temperatur kann durch ein Analogsignal variiert werden. Beide Parameter wurden durch Steuergeräte, die mit dem Messrechner verbunden waren, eingestellt. Dadurch konnte der Parameterwechsel automatisiert werden.
} 

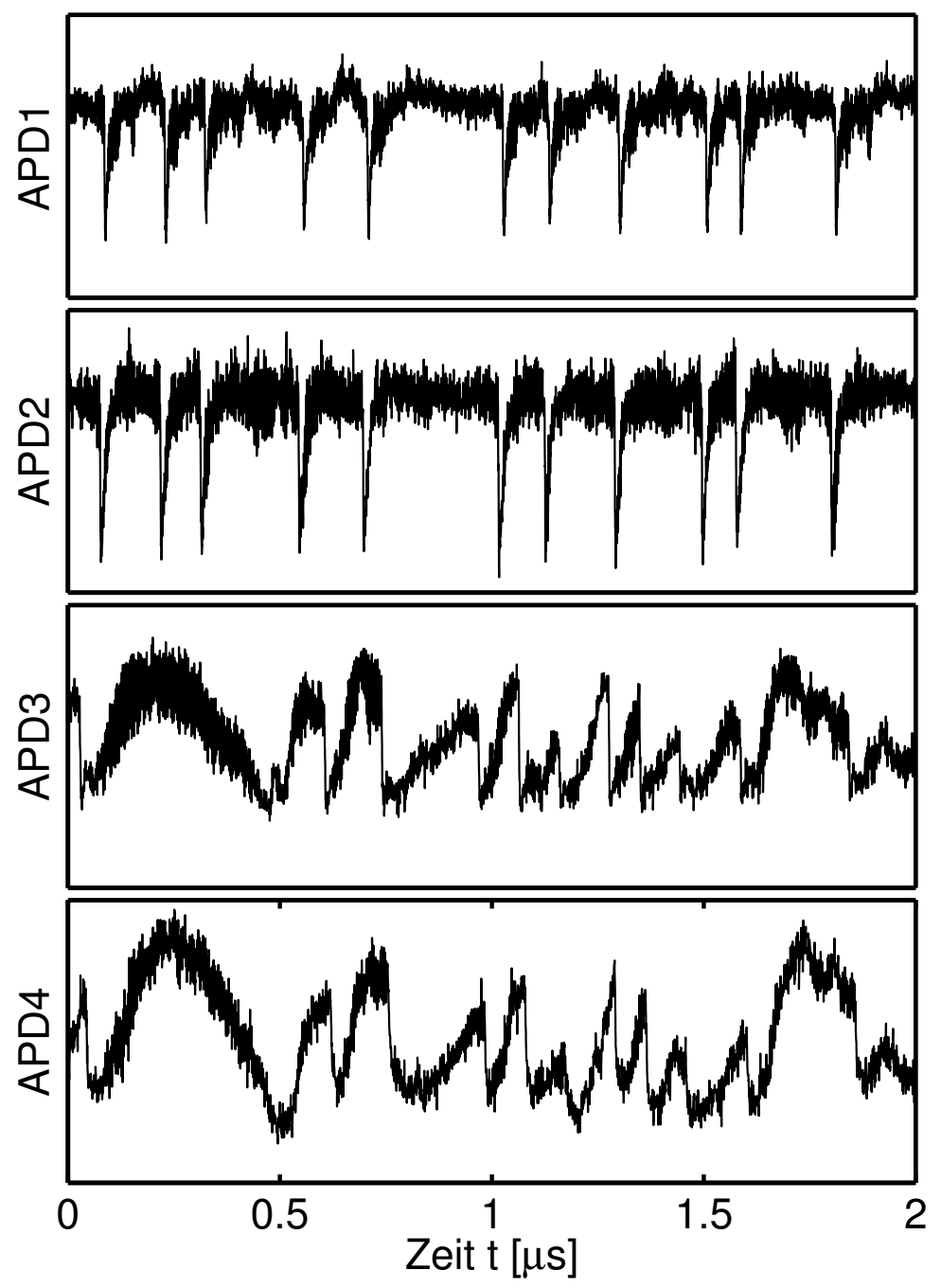

Abbildung 5.23: Auftragung der Intensitätssignale aller vier Detektoren APD1-APD4 (vgl. Abb. 5.21) im Bereich der Synchronisation der LFFDynamik, $\lambda=680.85 \mathrm{~nm}, T_{1}=16.37^{\circ} \mathrm{C}$. 
sität nicht vollführt muss diese Dynamik zwingender Weise von einer anderen aktive Mode stammen.

Leider war es mit der zur Verfügung stehenden Technik experimentell nicht möglich hier genauere Untersuchung zum Wechselspiel der verschiedenen Moden vorzunehmen. In der Numerik ist dies jedoch problemlos möglich und wird in Abschnitt 6.6 beschrieben.

\subsection{Synchronisation und Antisynchronisation der LFJ- Dynamik}
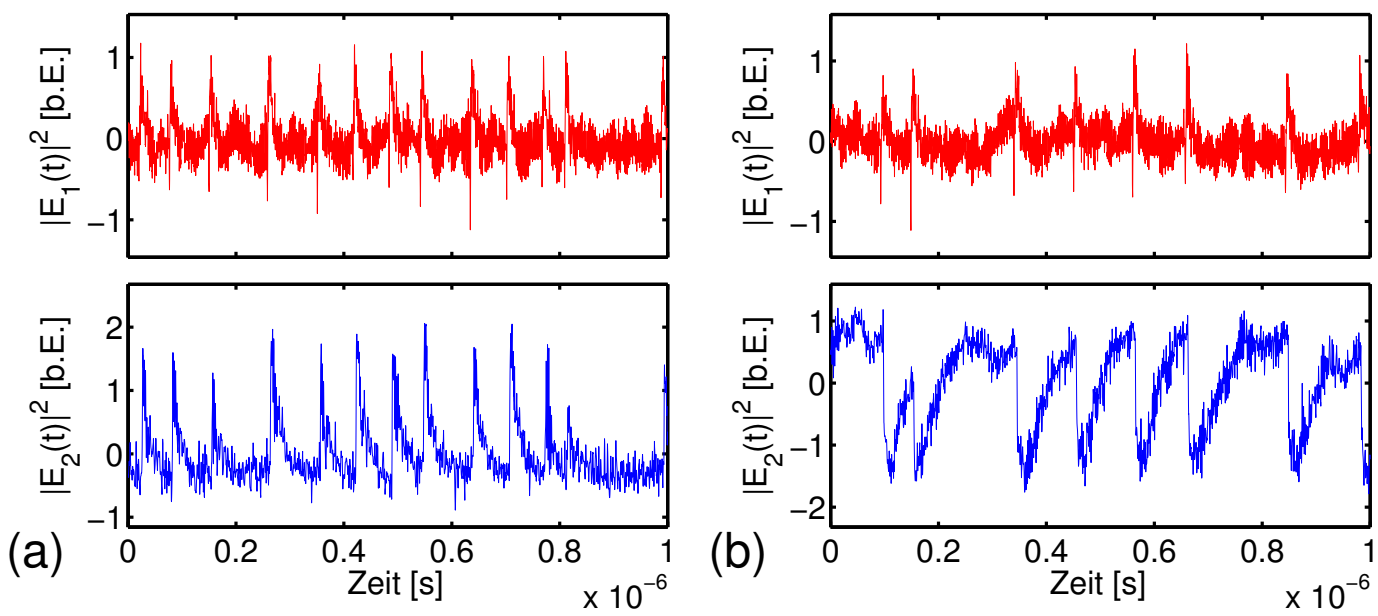

Abbildung 5.24: Auftragungen der Intensität für (a) das Synchronisationsgebiet und (b) das Antisynchronisationsgebiet bei LFJ-Dynamik. Parameter: $I_{2}=34.4 \mathrm{~mA}, T_{1}=19.4^{\circ} \mathrm{C}, T_{2}=20.48^{\circ} \mathrm{C}$, (a) $I_{1}=56.3 \mathrm{~mA}$, (b) $I_{1}=50.8 \mathrm{~mA}$.

Die Synchronisation der LFJ-Dynamik gelang im Experiment lediglich für den Fall ohne externen Resonator im Empfangssystem (Abbildung 5.24). Mit externem Resonator im Empfangssystem konnte die Eigendynamik dieses Systems nicht durch das eingekoppelte Licht des treibenden Systems beeinflusst werden. Der Grund hierfür liegt sehr wahrscheinlich in der zu geringen Kopplungsstärke. Zur Erzeugung der LFJDynamik im treibenden System ist es erforderlich den variablen Strahlteiler M1 auf eine relativ hohe Reflektivität einzustellen. Hierdurch wird jedoch auch die Kopplungsstärke reduziert, die Beeinflussung des Empfangssystems war so vermutlich nicht mehr möglich. 

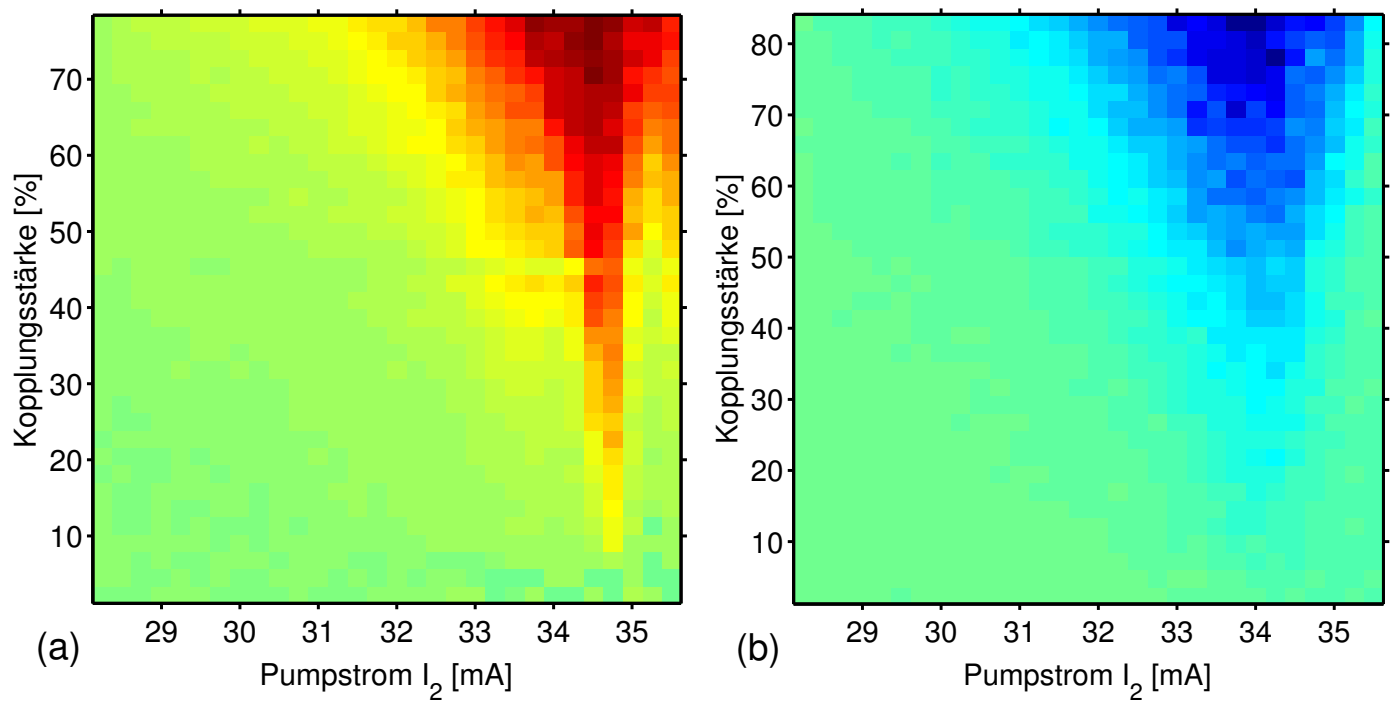

Abbildung 5.25: Auftragung des Synchronisationsindexes bei Variation Kopplungsstärke $\rho$ und des Diodenstroms $I_{2}$ bei LFJ-Dynamik. Parameter: $T_{1}=19.4^{\circ} \mathrm{C}, T_{2}=20.44^{\circ} \mathrm{C}$, (a) $I_{1}=55.5 \mathrm{~mA}$, (b) $I_{1}=50.5 \mathrm{~mA}$.

\subsubsection{Einfluss der Kopplungsstärke}

In Abbildung [5.25] ist der Synchronisationsindex über dem Kopplungsparameter $\rho$ und dem Pumpstrom $I_{2}$ aufgetragen. Im Gegensatz zur LFF-Dynamik gibt es hier, abhängig von den anderen festen Parametern $I_{1}, T_{1}, T_{2}$ nur eine Synchronisationsart. In Auftragung 5.25a ergeben die festen Parameter Synchronisation, in Auftragung 5.25] Antisynchronisation.

\subsubsection{Parameterbereiche der Synchronisation und Antisynchronisa- tion}

Abbildung 5.26 zeigt den Synchronisationsindex für die Variation der Parameter $I_{1}, T_{1}$ des treibenden Lasers. Die Struktur aus direkt benachbarten Gebieten der Synchronisation und Antisynchronisation ist sehr ähnlich zu der korrespondierenden Auftragungen der LFF-Dynamik (Abb. 5.14). Der Verlauf im Parameterraum sowie die Wiederholung dieser bandartigen Struktur ist ebenfalls mit der LFF-Dynamik vergleichbar. Die Reihenfolge der Synchronisationsgebiete ist jedoch genau umgekehrt: innerhalb einer Bandstruktur gelangt man durch Erhöhung des Pumpstromes oder der Temperatur des treibenden Lasers aus dem antisynchronen Bereich in den synchronen, dies ist für die LFF-Dynamik genau umgekehrt. 


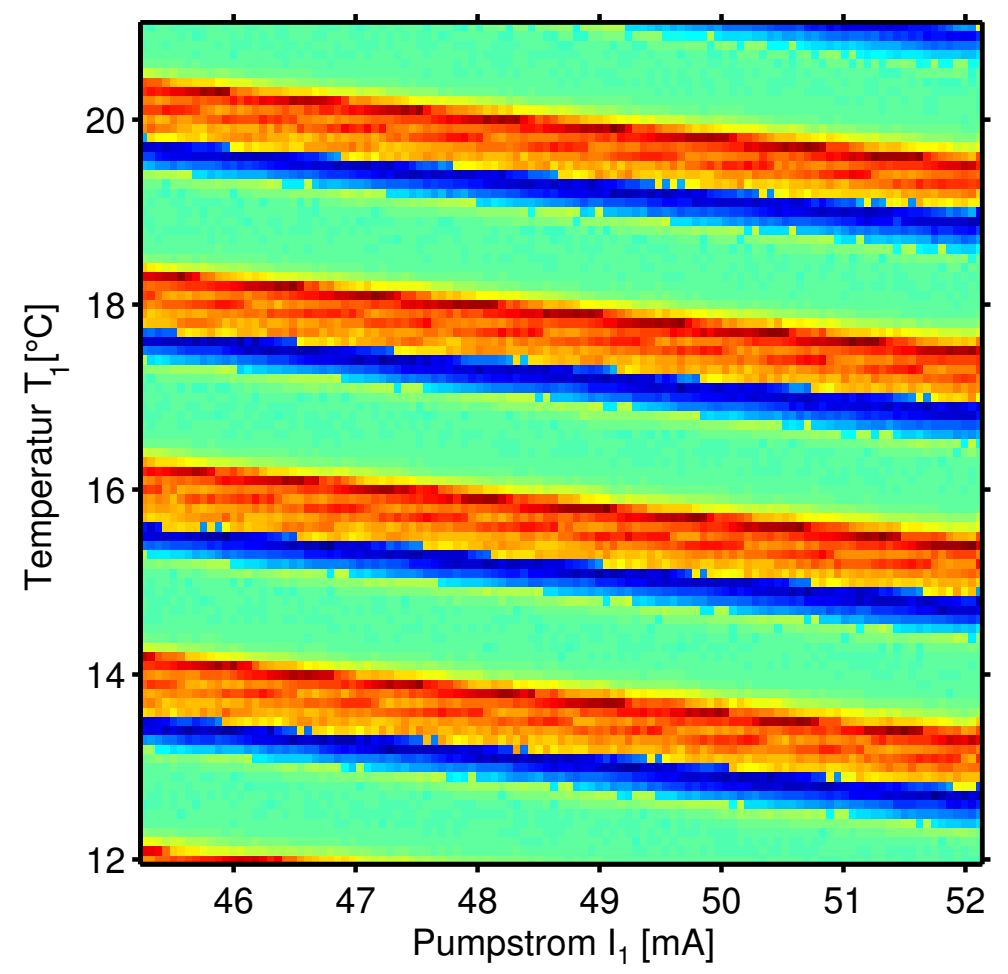

Abbildung 5.26: Auftragung des Synchronisationsindexes farbkodiert für verschiedene Pumpströme $I_{1}$ und Temperaturen $T_{1}$ des empfangenden Lasers bei LFJ-Dynamik im treibenden Laser. Kein externer Resonator im Empfangssystem. 


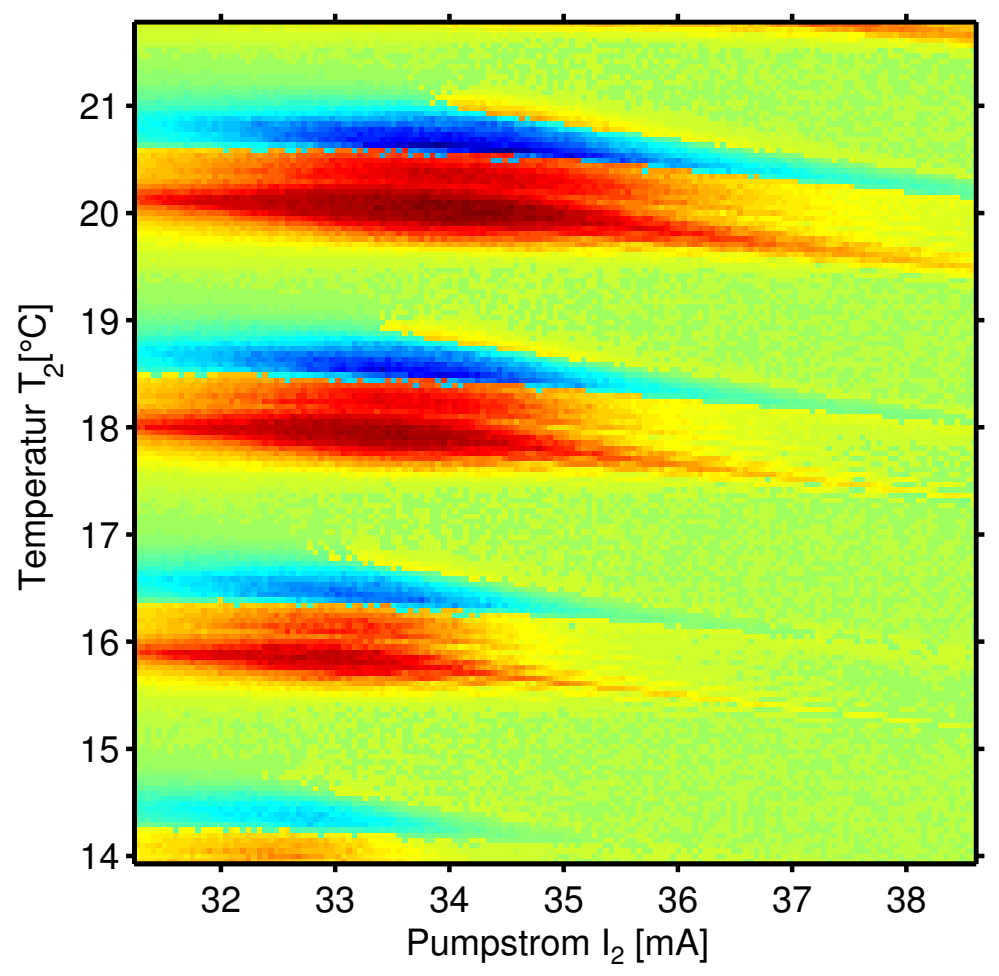

Abbildung 5.27: Auftragung des Synchronisationsindexes farbkodiert für verschiedene Pumpströme $I_{2}$ und Temperaturen $T_{2}$ des empfangenden Lasers bei LFJ-Dynamik im treibenden Laser. Kein externer Resonator Empfangssystem. 
Abbildung 5.27 zeigt den Synchronisationsindex für die Variation der Parameter $I_{2}, T_{2}$ des empfangenden Lasers. Auch hier zeigt sich diese Umkehrung der Synchronisationsreihenfolge gegenüber der gleichen Aufnahme für die LFF-Dynamik in Abbildung 5.13. In der Abbildung 5.27 fällt weiter auf, dass die Länge der Bänder mit dem Anstieg der Temperatur in Bezug auf den Pumpstrom $I_{2}$ zunimmt. Dieser Effekt ist ansatzweise auch in der korrespondierenden Auftragung für die LFF-Dynamik erkennbar (die Breite der Synchronisationsbereiche wird zu kleinen und großen Pumpströmen geringer, verschwindet jedoch in Abb. 5.13 nicht ganz).

Die Pumpstromabängigkeit der Trennlinie zwischen Synchronisationsgebiet und Antisynchronisationsgebiet ist in dieser Auftragung kaum vorhanden, ein Übergang von Synchronisation zu Antisynchronisation konnte demnach auch in der Auftragung für die Kopplungsabhängigkeit (Abb. 5.25) nicht vorkommen.

\subsubsection{Messung des optischen Leistungsspektrums}

Auch für den Fall der LFJ-Dynamik habe ich das Spektrum beider Laser nacheinander mit dem in Abbildung [5.15] skizzierten Aufbau für die Variation der Temperatur aufgenommen (Abbildungen 5.26 und 5.27). Im treibenden Laser sind bei dieser Dynamik offensichtlich wesentlich mehr Moden aktiv, als bei LFF-Dynamik. Dies hängt vermutlich mit dem deutlich höheren Pumpstrom von $I_{1}>50 \mathrm{~mA}$ zusammen 5 . Sehr gut ist in dieser Auftragung das langsame Anwachsen der Wellenlänge der einzelnen Moden mit steigender Temperatur zu erkennen. Dies war bei den Spektrogrammen für die LFFDynamik in Abschnitt 5.3.4 so nicht zu erkennen. Das Spektrum des Empfangslaser in Abbildung [5.27 zeigt ebenfalls sehr viele aktive Moden. Die Synchronisationsgebiete sind auch für diesen Fall im Spektrum auszumachen, wenn auch mit einem deutlich geringeren Kontrast.

Wegen der hier sehr zahlreich anschwingenden Moden, ist eine Ausschnittvergrößerung wie bei der LFF-Dynamik nicht sehr aussagekräftig. Interessanterweise zeigt die in Abschnitt 5.3.5 für die LFF-Dynamik verwendete Messmethode mittels des Monochromators einen interessanten Nebeneffekt. Durch die geringere Frequenzauflösung werden gerade diese sehr dicht beieinander liegenden Moden verwischt. Lediglich die stärkeren sind - mit einem geringen Signal-Rauschabstand - verhältnismäßig gut zu erkennen. Dies zeigen die vorweggenommenen Ausschnitte aus Abbildung [5.31] in Abbildung 5.30, Sehr gut sind die übereinstimmenden Modenfrequenzen beider Laser im

\footnotetext{
${ }^{5}$ Die Moden hoher Intensität wurden hierbei überbelichtet, um auch die schwächeren Moden noch zu detektieren.
} 


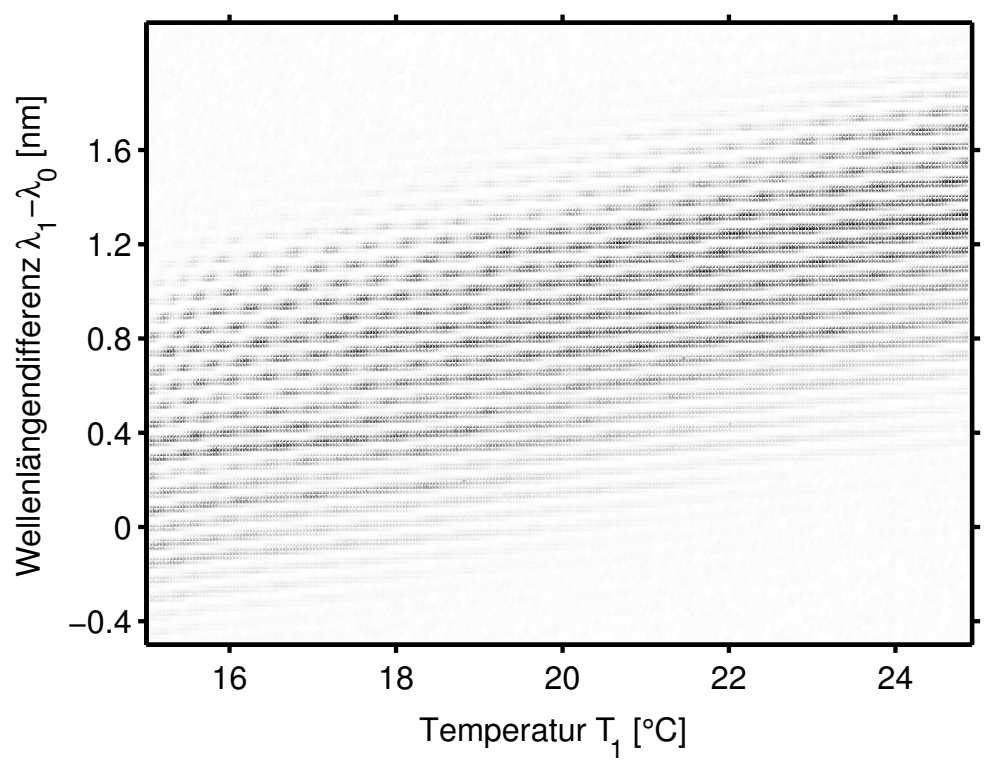

Abbildung 5.28: Auftragung des Leistungsspektrums des Lichtes des treibenden Lasers farbkodiert über der Temperaturen $T_{1}$ bei LFJ-Dynamik.

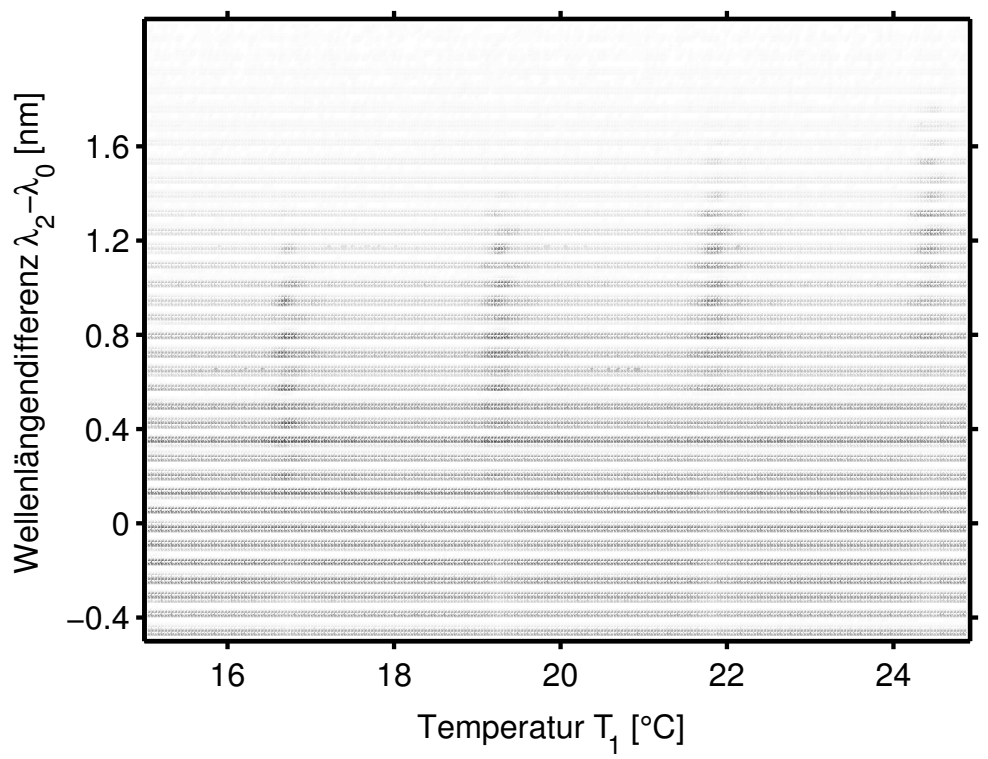

Abbildung 5.29: Auftragung des Leistungsspektrums des Lichtes des empfangenden Lasers farbkodiert über der Temperatur $T_{1}$ bei LFJ-Dynamik des treibenden Lasers. 
Bereich der Antisynchronisation zu erkennen (blaue Farbkodierung im Balken oberhalb beider Auftragungen in Abbildung [5.30).

Genau wie bei der LFF-Dynamik im Synchronisationsbereich bildet der empfangende Laser das Spektrum des treibenden Laser im Antisynchronisationsbereich für einen kleinen Parameterbereich nach. Innerhalb dieses Bereiches werden auch die Modensprünge reproduziert. An den Enden dieses Bereiches vollführt der treibende Laser ebenfalls einen Modensprung aus, diesem folgt der empfangende Laser jedoch nur mit sehr stark verringerter Intensität. Dieser Bereich ist in der Abbildung [5.30 nur sehr schwach erkennbar, da in der Auftragung in Wirklichkeit nicht die Intensität sondern die Fluktuationsstärke (Spitze-Spitze-Wert des Detektorsignals) dargestellt wird (siehe Erläuterung des Messaufbaus in Abschnitt [5.3.5).

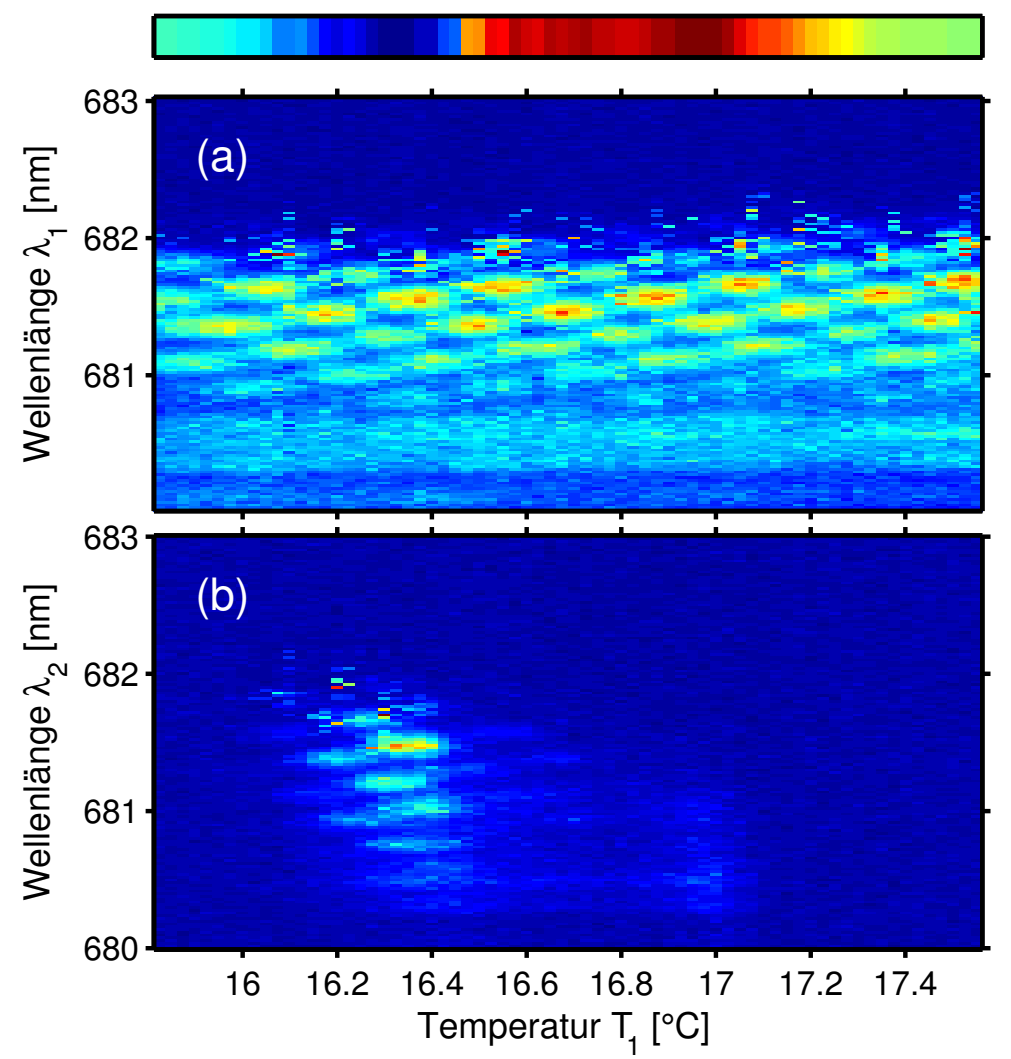

Abbildung 5.30: Detaillierte Betrachtung des optischen Leistungsspektrums bei Übergang von Anti-Synchronisation zu Synchronisation für den Fall der LFJ-Dynamik im treibenden Laser.

Alle diese Effekte stimmen sehr gut mit dem Verhalten bei LFF-Dynamik überein, nur sind das Synchronisationsgebiet und das Antisynchronisationsgebiet genau vertauscht.

Sehr schwach ausgeprägt ist in der Auftragung [5.30] bei ca. $T_{1}=17^{\circ} \mathrm{C}$ eine Reaktion 
des Empfangslasers im Bereich der Synchronisation auszumachen. Genau an dieser Stelle zeigt der Synchronisationsindex sehr hohe positive Werte (dunkelrote Farbgebung im Balken oberhalb beider Auftragungen in Abbildung [5.30). Dieser Effekt ist auch in den Abbildungen 5.26 und 5.27 zu erkennen: Am Außenrand des Synchronisationsgebiets bei verhältnismäßig großem Abstand zum Antisynchronisationsgebiet zeigt der Synchronisationsindex höhere positive Werte als im übrigen Synchronisationsbereich.

\subsubsection{Modensynchronisation}

Die Modensynchronisation lässt sich für die LFJ-Dynamik mit dem in Abbildung [5.21] dargestellten Aufbau nachweisen. Als problematisch hat sich jedoch die sehr geringe Intensität des Lichtes des Empfangslasers erwiesen, da dieser sehr dicht an der Laserschwelle betrieben werden muss. Einzige Möglichkeit zur Gewinnung von genug Licht zur Detektion mit den APDs nach der optischen Filterung war die Vergrößerung des Eingangsspaltes des Monochromators. Dieser definiert jedoch auch die Frequenzauflösung der optischen Filterung, wodurch die Messungen in Abbildung 5.31 an der Auflösungsgrenze der auftretenden Modenfrequenzen durchgeführt wurde.

Trotz des dadurch relativ schlechten Signal-Rausch-Verhältnis ist doch recht deutlich ein sehr ähnliches Verhalten zur LFF-Dynamik in Abbildung 5.22 zu erkennen. Wie bereits bei der Detailbetrachtung in Abbildung 5.30 zu beobachten war, zeigt sich lediglich in Bereich der Antisynchronisation eine signifikante Reaktion des Empfangslasers (Abb. 5.31b) auf das Kopplungssignal. Der Synchronisationsindex in Abbildung 5.31; zeigt überraschenderweise positive Werte (rote Farbgebung) für die im Empfangslaser aktiven Moden an. Das bedeutet, dass die Einzelmoden im Antisynchronisationsgebiet synchronisiert sein müssten.

Für die Parameterkombination $\lambda=681.5 \mathrm{~nm}, T_{1}=19^{\circ} \mathrm{C}$ zeigt Abbildung 5.32 die Intensitätssignale aller vier Photodetektoren APD1-APD4 für dieses sehr überraschende Ergebnis. Das oben beschriebene Phänomen ist hier aber, trotz des relativ schlechten Signal-Rauschabstand für die Detektoren APD3 und APD4, ohne Zweifel erkennbar. Die Intensitätssignale APD1 und APD2 sind antisynchron zueinander, jedoch gleichzeitig ist die durch APD3 und APD4 beobachtete Einzelmode beider Laser synchronisiert. 


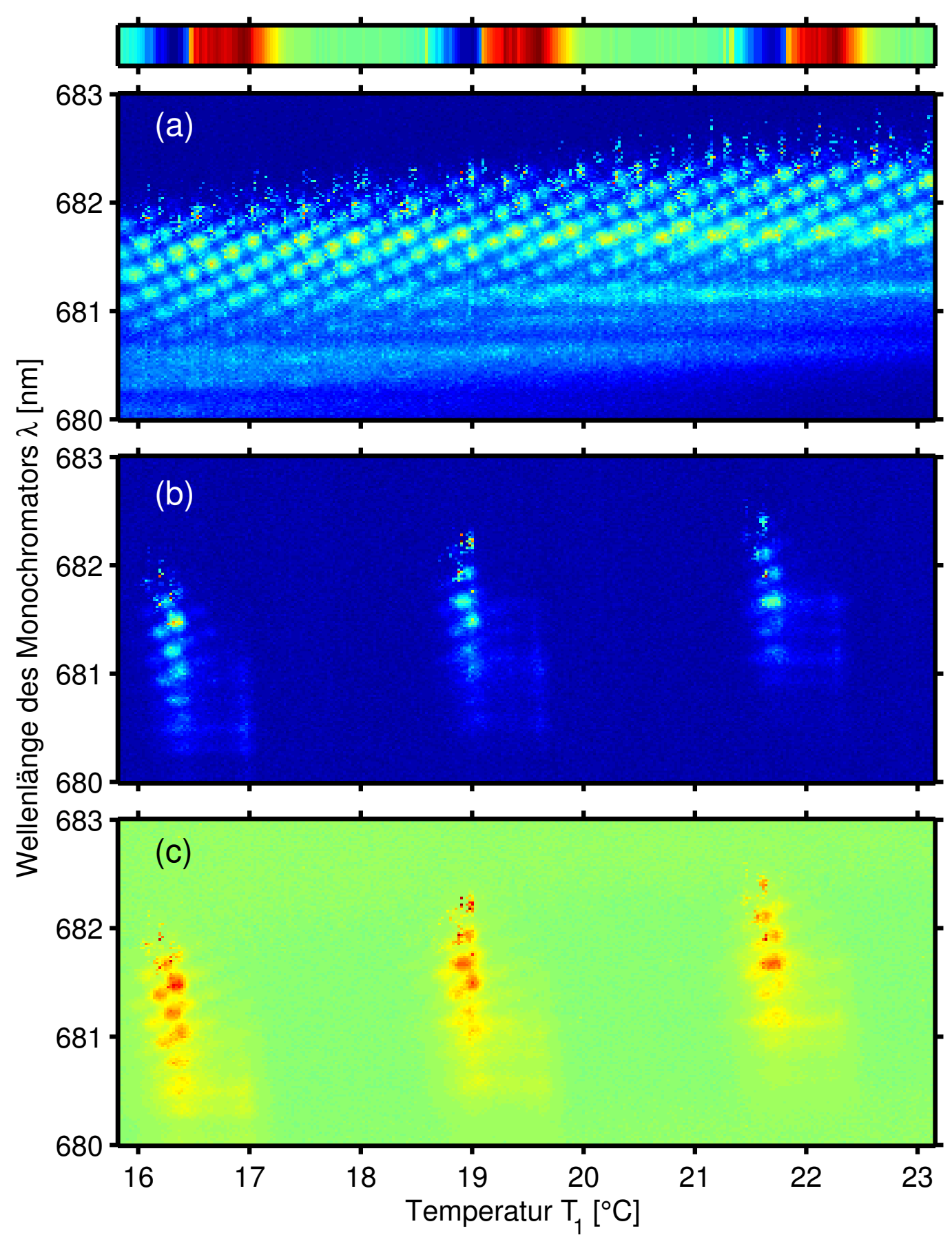

Abbildung 5.31: Modensynchronisation bei LFJ-Dynamik im treibenden Laser. (a) Fluktuationsstärke des treibenden Lasers, (b) Fluktuationstärke des empfangenden Lasers, (c) Synchronisationsindex der gefilterten Laserintensitäten. Der Balken oberhalb der Auftragungen zeigt den Synchronisationsindex der Gesamtintensitäten. 

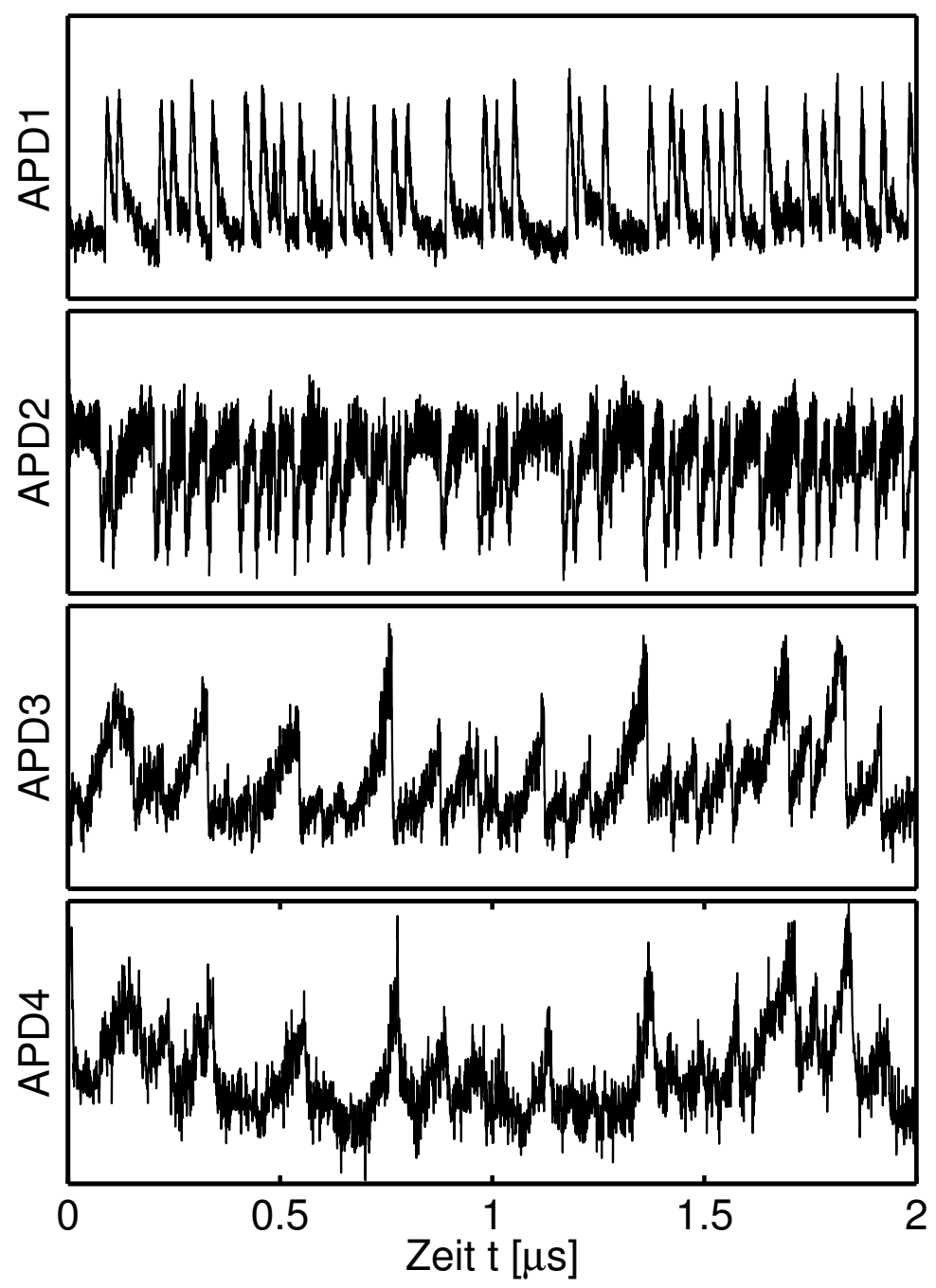

Abbildung 5.32: Auftragung der Intensitätssignale aller vier Detektoren APD1-APD4 (vgl. Abb. [5.21) im Bereich der Antisynchronisation der LFJDynamik. 


\subsection{Modensynchronisation im Kohärenzkollaps}

Im Bereich des Kohärenzkollapses ist eine Bestimmung der Synchronisation anhand der Kreuzkorrelation der Gesamtintensitäten nicht mehr möglich, da die langsamen Intensitätseinbrüche oder Intensitätssprünge der Hüllkurve des elektrischen Feldes verschwinden (siehe Abschnitt 3.1). Die wesentliche schnellere Pulsdynamik ist, wie bereits erläutert, mit den hier zur Verfügung stehenden Detektoren nicht auflösbar. Während der Untersuchung der Modensynchronisation mit dem in Abbildung [5.21] beschriebenen Aufbau stellte sich jedoch heraus, dass das optisch gefilterte Intensitätssignal im Bereiche des Kohärenzkollaps wesentlich langsamere Fluktuationen zeigte und problemlos mit dem Detektoren aufzunehmen war 6 . Abbildung 5.33 zeigt dies in der gewohnten Auftragungsform. Der Synchronisationsindex der optisch nicht gefilterten Intensitäten, der farbkodiert oberhalb der drei Auftragungen als Balken dargestellt ist, zeigt sogar noch eine sehr schwache positive (rot) bzw. negative (blau) Korrelation im Bereich der Modensynchronisation an. Diese Korrelationen sind jedoch extrem schwach (maximaler Korrelationskoeffizient $\approx 0.15$ ). Erst durch die sehr deutlich positiven Synchronisationsindizes in Auftragung 5.33k bei diversen Moden kann von Synchronisation zwischen den Lasern ausgegangen werden.

Sehr gut ist die Synchronisation der Modensprünge zu erkennen. Der Empfangslaser zeigt in Auftragung 5.33b im Gegensatz zum LFF- bzw. LFJ-Regime einen relativ breiten Synchronisationsbereich, in dem sich die Modenfrequenzen in derselben Art und Weise verhalten wie beim treibenden Laser in Auftragung 5.33 a. In diesem Bereich verlaufen auch die Zeitsignale der Moden synchron zwischen beiden Lasern, wie die Auftragung [5.33] des Synchronisationsindexes belegt.

Zwischen den Synchronisationsgebieten zeigt der Empfangslaser in Auftragung [5.33b sehr deutliche spontane Modensprünge. Bei den (zufällig gewählten) Parameterwerten des Empfangslasern erfolgt die Umschaltung zwischen zwei Modenfrequenzen so langsam, das diese im Verlaufe der Messung gut erkennbar aufgezeichnet wurde.

\subsection{Synchronisation der spontanen Modensprünge}

Um zu untersuchen, ob auch diese spontanen Modensprünge zwischen zwei Lasern synchronisierbar sind, habe ich denselben Messaufbau wie er in Abbildung [5.21] skizziert ist, verwendet. Lediglich der Spiegel M1 zur Realisierung des externen Resonators

\footnotetext{
${ }^{6}$ Diese langsamen Fluktuationen stammen nicht aus der Dynamik des Kohärenzkollapses. Die sind vermutlich auf die Modendynamik zurückzuführen.
} 


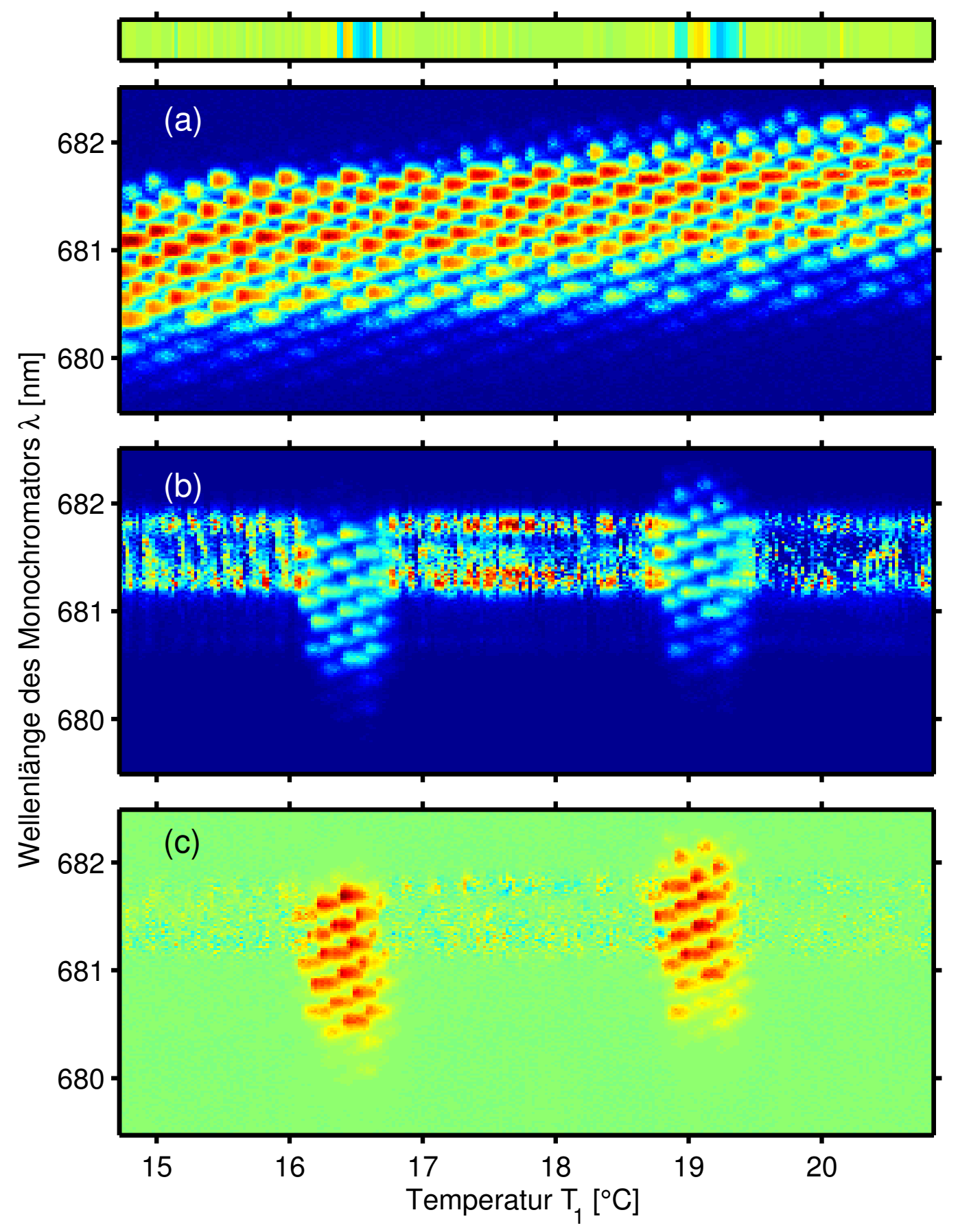

Abbildung 5.33: Modensynchronisation im Bereich des Kohärenzkollaps. (a) Fluktuationsstärke des treibenden Lasers, (b) Fluktuationstärke des empfangenden Lasers, (c) Synchronisationsindex der gefilterten Laserintensitäten. Der Balken oberhalb der Auftragungen zeigt den Synchronisationsindex der Gesamtintensitäten. 
für den treibenden Laser entfiel. Im Falle des externen Resonators traten die Modensprünge in sehr regelmäßigen Bereichen, verteilt über den gesamten Messbereich auf. Für spontane Modensprünge gilt dies nicht, sie treten nur auf, wenn eine weitere interne, longitudinale Resonatormode in den Bereich positiven Gewinns des laseraktiven Materials gerät. Wegen der geringen Größe des internen Resonators (ca. 300 $\mu m$ ) ist dies im betrachteten Messbereich wesentlich seltener der Fall.

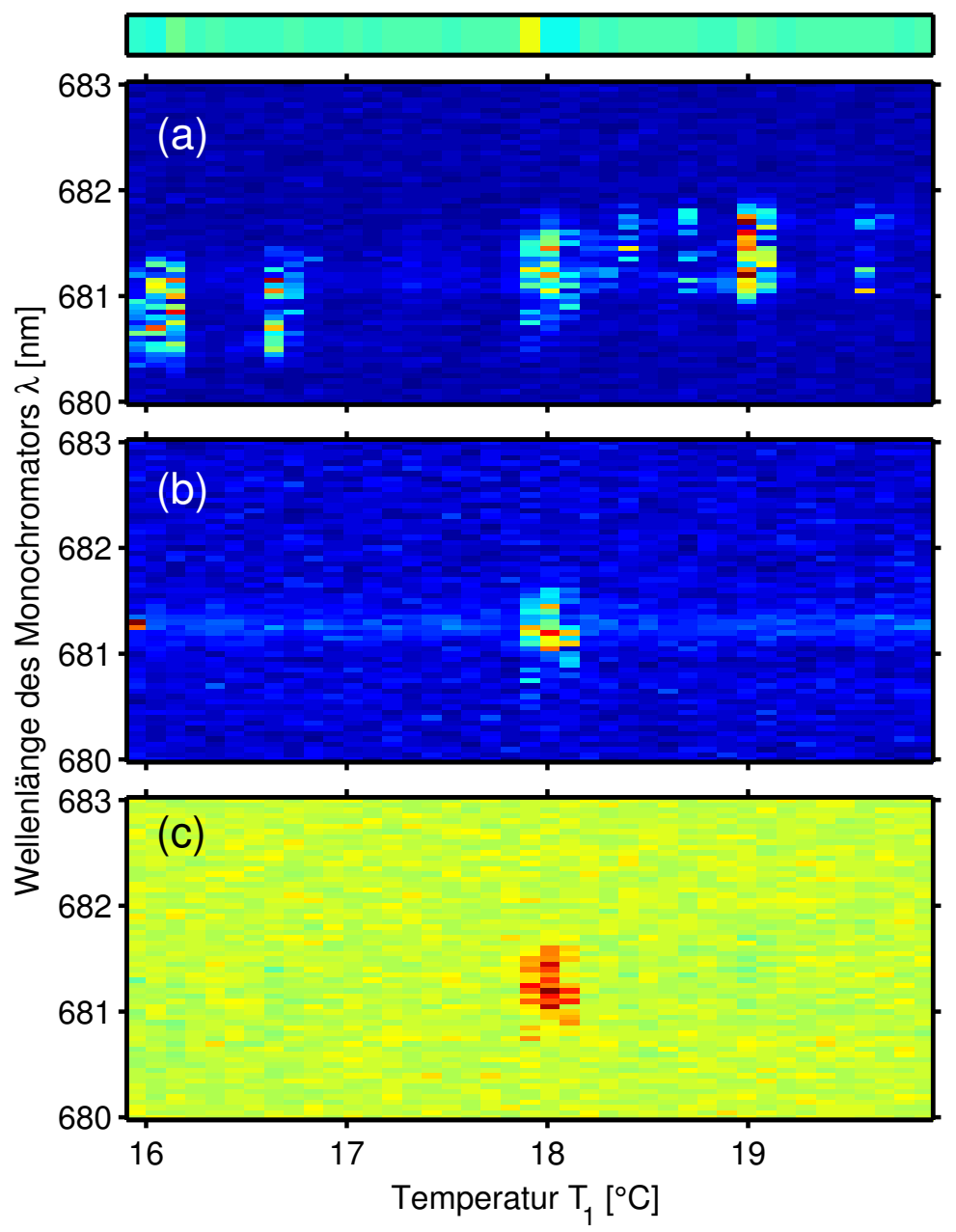

Abbildung 5.34: Modensynchronisation der spontanen Modensprünge. (a) Fluktuationsstärke des treibenden Lasers, (b) Fluktuationstärke des empfangenden Lasers, (c) Synchronisationsindex der gefilterten Laserintensitäten. Der Balken oberhalb der Auftragungen zeigt den Synchronisationsindex der Gesamtintensitäten.

Abbildung 5.34 zeigt das Spektrogramm des treibenden Lasers 5.34a, das des empfangenden Laser 5.34b sowie den farbkodierten Synchronisationsindex der Gesamtintensitäten der Laser (Balken oberhalb der Auftragungen) als auch den der optisch 
gefilterten Laserintensitäten 5.34t.

Wie zu erwarten, zeigt der Synchronisationsindex der Gesamtintensitäten der Laser praktisch keinen von Null verschiedenen Wert. Das Spektrum des treibenden Lasers zeigt auch nur bei wenigen verschiedenen Temperaturen signifikante Fluktuationen. Die Fluktuation bei $T_{1} \approx 18^{\circ} \mathrm{C}$ tritt auch im empfangenden Laser auf und zeigt einen hohen, positiven Synchronisationsindex. Eine Synchronisation der spontanen Modensprünge ist offenbar möglich, wie auch die Zeitsignale der beiden optische gefilterten Laserintensitäten in Abbildung [5.35 zweifelsfrei zeigen.

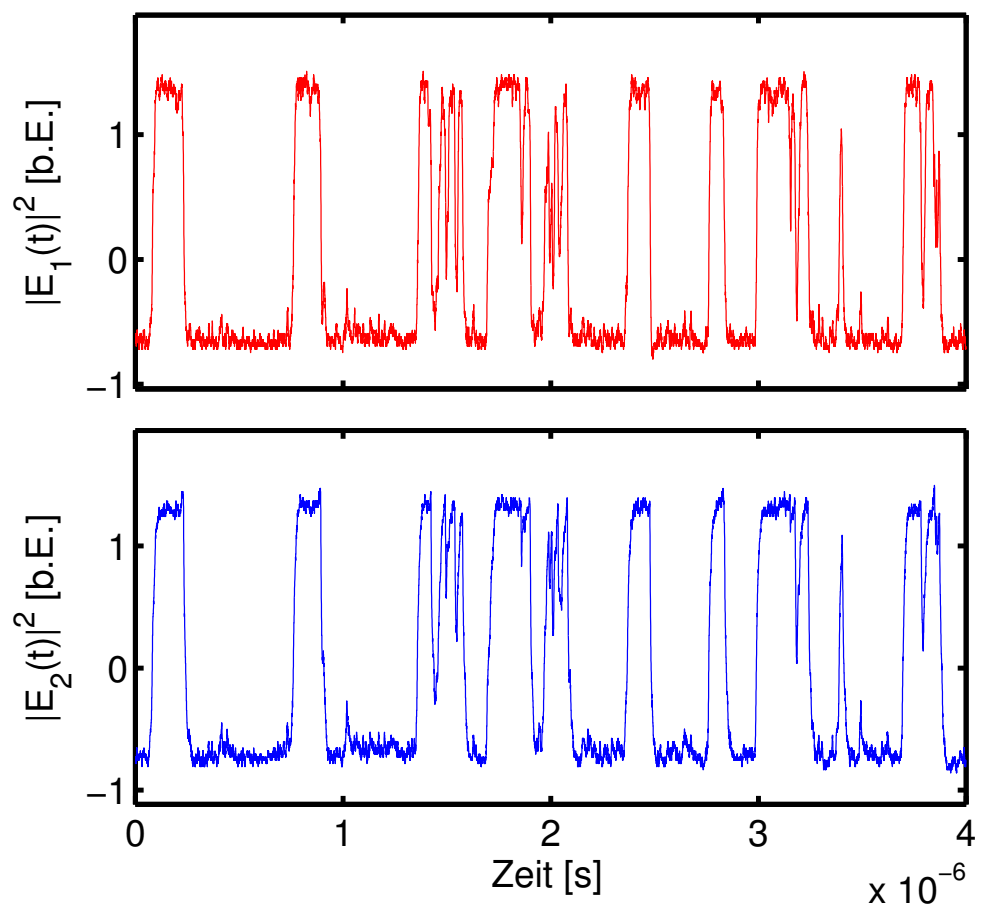

Abbildung 5.35: Optisch gefilterte Intensitätssignale beider Laser bei Synchronisation der spontanen Modensprünge.

\subsection{Antisynchronisation der spontanen Modensprünge}

Sehr überraschend zeigte sich auch in sehr seltenen Fällen eine Antisynchronisation der Modensprünge. Die Parameterbereiche, in denen dieses Phänomen auftrat sind extrem klein und wurden bisher nur durch Zufall gefunden. Häufig konnte diese Antisynchronisation nur für wenige Augenblicke aufrecht erhalten werden. Die Vermutung liegt hierbei nahe, dass einige der ungenügend kontrollierbaren Parameter des experimentellen Aufbaus wegdriften. Dadurch war es bisher nicht möglich eine genaueres 
Studium dieses Phänomens durchzuführen. Nichts destotrotz habe ich dieses Phänomen diverse Male für kurze Momente (bis zu einigen Minuten) beobachten können. Abbildung [5.36] zeigt ein Beispiel der optisch gefilterten Laserintensitäten.

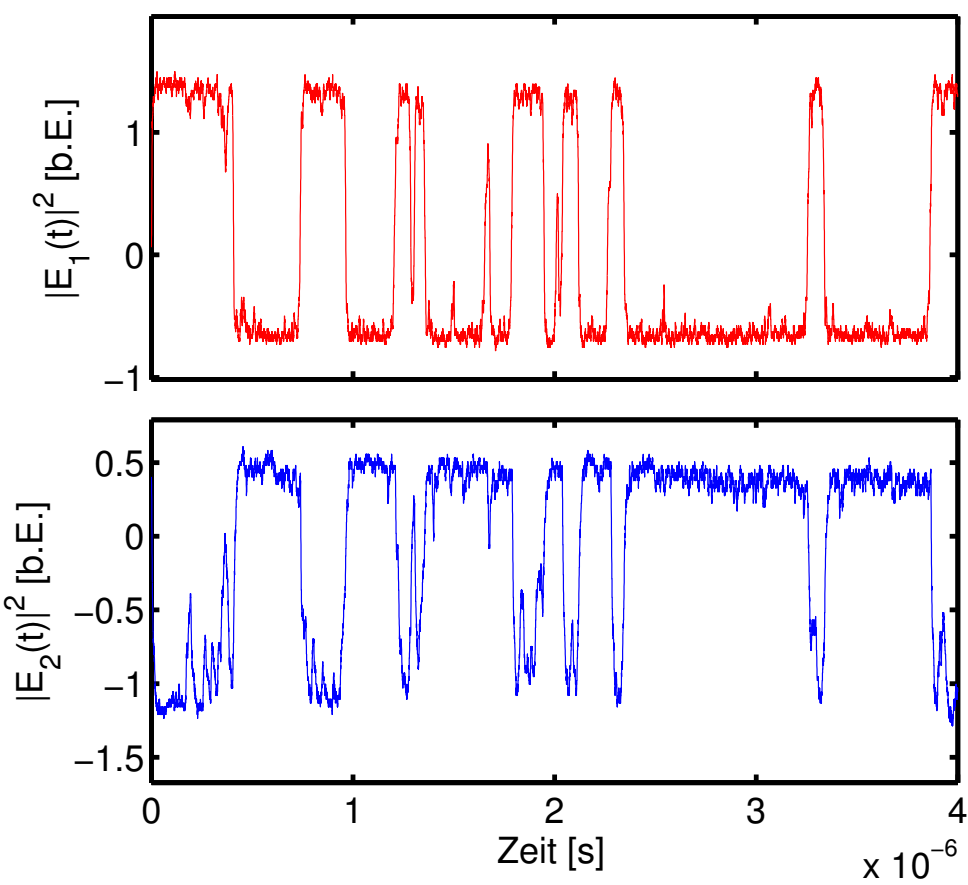

Abbildung 5.36: Optisch gefilterte Intensitätssignale beider Laser bei Antimodensynchronisation der spontanen Modensprünge.

Zwar reichte die Zeit in der diese Antisynchronisation bestand, nicht aus, um aufwendigere Messungen durchzuführen. Die bisherige experimentelle Erfahrung mit dem Phänomen lässt jedoch den im folgenden kurz erläuterten Mechanismus vermuten.

Die Auftragung 5.34 legt nahe, dass die im Bereich der im treibenden Laser auftretenden Modensprünge zwischen genau zwei Moden stattfinden. Mit dem vorhandenen Monochromator wird das Licht genau einer Mode hindurchgelassen und die Intensitätskurve zeigt dann beispielsweise den in der Abbildung 5.36 oben gezeigten Verlauf. Da die Energie zwischen den Moden hin- und her transportiert wird und die Gesamtintensität konstant bleibt, muss die nicht beobachtete Mode ein genau invertiertes Intensitätssignal zu der Auftragung in der Abbildung zeigen.

Nun nehme ich an, dass eine (identische) Synchronisation wie sie in vorherigen Abschnitt gezeigt wurde zwischen zwei Laserdioden stattfindet. Im Allgemeinen kann davon ausgegangen werden, dass die Modenfrequenzen beider Laserdioden näherungsweise gleich sein müssen, um Synchronisation zu ermöglichen. Der Auftragung [5.34] ist dies auch zu entnehmen. In einigen von mir durchgeführten Messungen konnte 
ich jedoch sehen, dass Synchronisation auch bei deutlicher Frequenzverschiebung der Moden im Empfangslaser stattfand. Dieses Phänomen war jedoch ebenso selten und instabil wie die Antisynchronisation und die zur Verfügung stehende Beobachtungszeit reichte nicht aus, um eine aussagekräftige Messung des Phänomens durchzuführen.

Wenn dieses Frequenzverschiebung als möglich und ausreichend groß erweist, so lässt sich damit direkt die in Abbildung [5.36] gezeigte Messung erklären. In Abbildung 5.37 ist diese Vermutung skizziert. In der Darstellung (a) ist schematisch das Spektrum des treibenden Lasers, gemessen mit relativ geringer Frequenzauflösung ähnlich dem Monochromator, aufgetragen. Die Intensitätssignale dieser beiden Moden des treibenden Lasers sind antikorreliert. In Darstellung (b) ist das schematisierte Spektrum des synchronisierten Empfangslasers dargestellt. Die Modenfrequenzen sind stark verschoben. Synchronisation beider Laser bedeutet, dass sowohl die Intensitäten der ersten Moden jedes Lasers, als auch die zweiten Moden beider Laser zueinander korreliert sind. D.h. auch, dass das Intensitätssignal der zweiter Mode des treibenden Lasers mit der ersten Mode des Empfangslasers antisynchronisiert ist.

Durch die Verwendung von nur einem Monochromator können zeitgleich nur die optisch gefilterten Intensitätssignale derselben Frequenz aufgezeichnet und verglichen werden. Durch die begrenzte Frequenzauflösung des Monochromators gibt es jedoch Frequenzen im Spektrum zwischen beiden Moden, bei denen der Detektor für die optisch gefilterte Intensität des treibenden Lasers die zweite Mode detektiert, der Detektor für den empfangenden Laser jedoch das Licht der ersten Mode. Diese sind nach der obigen Überlegung antisynchron zueinander. Der Synchronisationsindex für das schematisierte Beispiel ist in Abbildung 5.37k zu erkennen.

Die Frequenzverschiebung ist in diesem Beispiel zur Verdeutlichung sehr groß gewählt. Bei Verringerung Frequenzverschiebung verkleinert sich sinngemäß der antisynchrone Bereich, bis er komplett verschwindet und nur noch identische Synchronisation zu messen ist.

Um diese Vermutung zu untermauern wäre es nötig, genau die in der Abbildung 5.37 skizzierten Intensitätskurven und den Synchronisationsindex zu messen. Bisher ist dies jedoch nicht gelungen. 

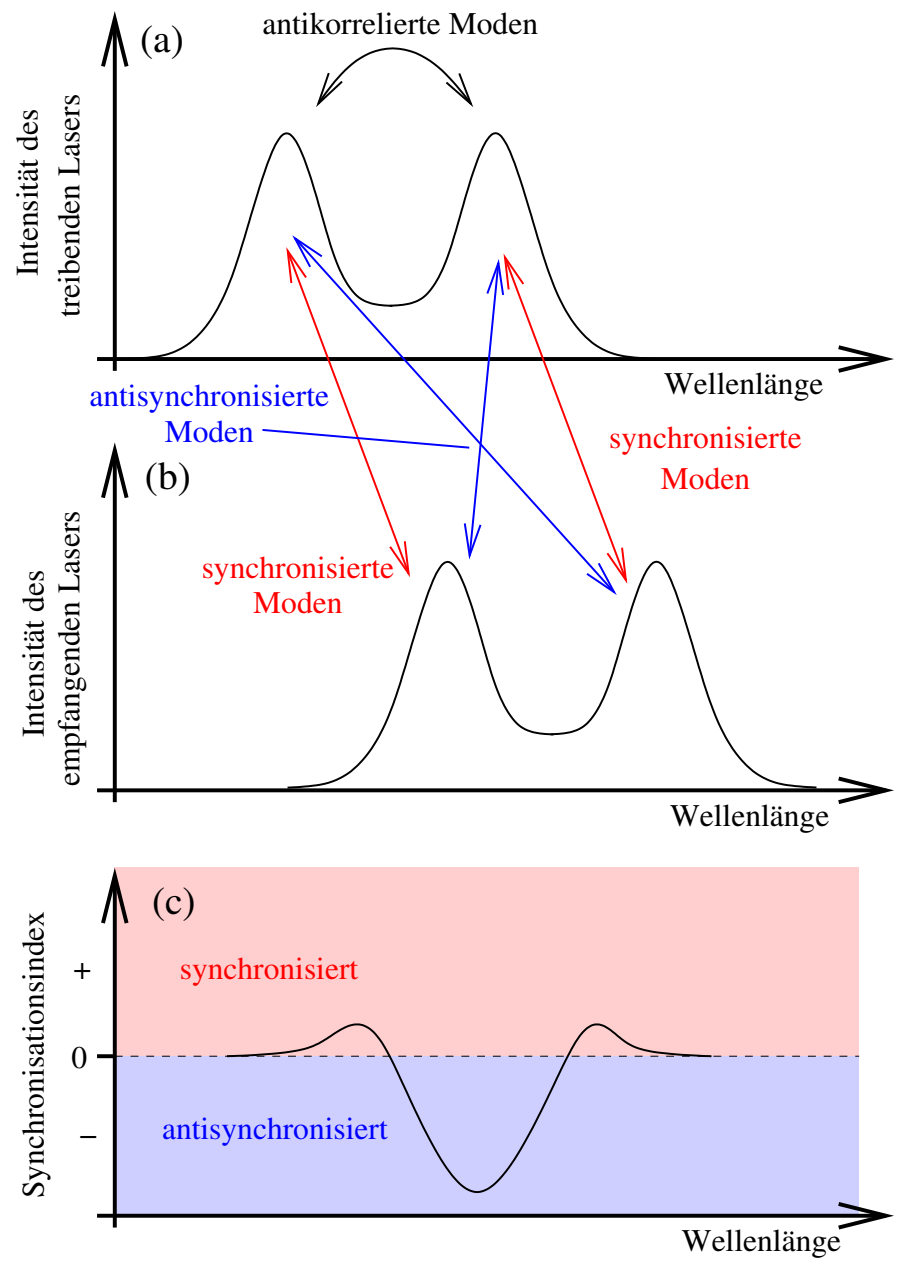

Abbildung 5.37: Skizze zur Erklärung der Antisynchronisation der Modenintensitäten. 



\section{Kapitel 6}

\section{Numerische Simulation der Lasersynchronisation}

In diesem Kapitel habe ich die Ergebnisse meiner numerischen Simulationen zusammengefasst. Ziel war es, ein Modell zu entwickeln, dass qualitativ gut mit den von mir experimentell beobachteten Phänomenen übereinstimmt. Dieses Kapitel stellt den experimentellen Ergebnissen des vorherigen Kapitels die numerischen gegenüber.

\subsection{Historischer Überblick}

Numerisch wurde schon Anfang der 90er Jahre des letzten Jahrhunderts die optische Kopplung von Lasern untersucht. Winful et al. konnten 1990 [86] die Synchronisation von drei gegenseitig gekoppelten Lasergleichungssystemen nach Gleichung (2.30) und (2.31) beschreiben. Die Kopplung wurde hier über die Feldgleichung erreicht. Synchronisation sowie raumzeitliches Chaos konnte damit erzeugt werden. In diesen Simulationen wurde keine Rückkopplung verwendet, die Dynamik kam durch die gegenseitigen Kopplung der drei Laser zustande.

Die erste numerische Simulation optisch gekoppelter Halbleiterlaser mit externem Resonator wurden 1996 von Mirasso et al. [47] vorgestellt. Durch die Wahl von unterschiedlichen Parametern für beide Systeme war jedoch keine perfekte identische Synchronisation möglich. Durch ein etwas verändertes Kopplungsschema konnten AnNovazzI-Lodi et al. 1997 identische Synchronisation in ihren numerischen Simulationen erreichen.

AHLERs et al. zeigten 1998 [4, 5] erstmals numerisch die identische Synchronisation der LFF-Dynamik. 


\subsection{Modellierung der Wellenlängenabhängigkeit}

Durch die im letzten Kapitel dargelegten Messungen ist es offensichtlich, dass die Lichtfrequenz der Laser bei der Erklärung vieler Effekte eine wichtige Rolle spielt. In den semiklassischen Lasergleichungen wurde die Lichtfrequenz jedoch komplett eliminiert, da ihr elektrisches Feld um viele Größenordnungen schneller fluktuiert als die aufgeprägte Amplitudendynamik 1 .

Alle im Experiment beobachteten Veränderungen der Lichtfrequenz beider Laser lassen sich so nicht direkt mit diesen Gleichungen simulieren, da es hier streng genommen keine Lichtfrequenz gibt. Wie die Vermutung in Abschnitt 5.3.3 nahelegt, ist jedoch nicht die absolute Lichtfrequenz von Interesse, sondern vielmehr die wesentlich langsamer fluktuierende Differenzfrequenz beider Laser.

Diese kann nun künstlich, also als weiterer Parameter, in die Gleichungen eingefügt werden. Ein so eingefügter Parameter ist jedoch von vornherein erst einmal unabhängig. Eine Abhängigkeit wie im Experiment beispielsweise von der Temperatur muss zusätzlich modelliert werden. Ein anderer Weg wäre eine komplette Simulation des Lasers inklusive der Lichtfrequenz und Modenstruktur und ist nur über die Lösung einer sehr komplizierten partiellen Differentialgleichung möglich. Bisher sind mehrere Veröffentlichungen zu einem solchen partiellen Gleichungssystem erschienen, jedoch konnte bisher noch niemand die Simulation der LFFs anhand eines solchen Modells zeigen. Akzeptiert man jedoch die Einschränkungen des im weiteren verwendeten LangKobayashi-Modells in Bezug auf die künstliche Modellierung der Differenzfrequenz, kann es viele der experimentell gefundenen Effekte wiedergeben.

Gleichung (3.11) beschreibt die komplexe Amplitude des elektrischen Feldes für jede beteiligte Mode. Durch die Lichtfrequenzänderung eines der Laser kommt es zu einer Frequenzdifferenz zwischen beiden Lasern, die sich wiefolgt auf die Kopplung auswirkt. Sei $\omega$ die Kreisfrequenz des treibenden Lasers und $\hat{\omega}$ die des empfangenden Lasers, dann gilt für die kohärente Überlagerung beider elektrischen Felder einschließlich der Lichtfrequenz:

$$
\hat{E}(t) e^{i \hat{\omega} t}+E(t) e^{i \omega t}=\left(\hat{E}(t)+E(t) e^{i(\omega-\hat{\omega}) t}\right) e^{i \hat{\omega} t}
$$

Betrachtet man also nur die komplexe Amplitude des elektrischen Feldes (eingeklammerter Term), tritt die Differenzfrequenz als Modulation $e^{i(\omega-\hat{\omega}) t}$ der Feldamplitude auf.

\footnotetext{
${ }^{1}$ Erreicht wurde dies durch die s.g. Slowly Varing Envelope Approximation - SVEA.
} 


\section{Wellenlängenabhängigkeit der Lasertemperatur und des Pumpstromes}

Die Differenzfrequenz zwischen beiden Lasern lässt sich im Experiment nicht frei wählen, sondern hängt sowohl von den Temperaturen als auch von den gewählten Pumpströmen beider Laser ab. Die Parameter der numerischen Simulation für die Differenzfrequenz $\omega-\hat{\omega}$ und den Pumpstrom $p$ müssen nun sinnvoll auf die im Experiment vorkommenden Parameter für Temperatur $T$ und Pumpstrom $I$ abgebildet werden. Erst dann ist es möglich zu entscheiden, inwieweit die Änderungen der Dynamik bei Änderung der Parameter im Experiment und in der numerischen Simulation qualitativ vergleichbar sind.

Die Abbildung des Pumpstromes $I$ im Experiment auf den Pumpstrom $p$ in der numerischen Simulation ist sehr einfach, da es sich bei $p$ um den normierten Pumpstrom, relativ zur Laserschwelle handelt. Es kann also angenommen werden, dass

$$
p=\frac{I}{I_{t h}}
$$

gilt mit $I_{t h} \approx 32 \mathrm{~mA}$ als Pumpstrom an der Laserschwelle im Experiment. Darüberhinaus hat jedoch der Pumpstrom $I$ im Experiment noch einen sehr erheblichen Einfluss auf die Wellenlänge. Dies lässt sich sehr schön in Abbildung 6.1] erkennen. Offensichtlich ändert sich die Wellenlänge der Moden mit dem Pumpstrom im wesentlichen in Sprüngen. Sehr ähnlich sieht der Einfluss der im Experiment einstellbaren Temperatur $T$ auf die Wellenlänge des Lasers aus, wie die Abbildung 6.1p zeigt.

Für das von mir verwendete qualitative Modell nehme ich vereinfachend an, dass sich die Wellenlänge linear mit dem Pumpstrom und der Temperatur ändert. Für die Wellenlängendifferenz bedeutet dies:

$$
\omega-\hat{\omega}=\Delta \omega_{T} \cdot(T-\hat{T})+\Delta \omega_{I} \cdot(I-\hat{I})
$$

Zur Ermittlung der Steigungen $\Delta \omega_{T}$ und $\Delta \omega_{I}$ verwende ich die Auftragungen aus Abbildung 6.1. Für eine ausgewählte Mode habe die Steigungen gemäß der weißen Linien bestimmt. Daraus ergeben sich folgende Werte:

$$
\begin{aligned}
\Delta \omega_{T} & \approx-2.12 \cdot 10^{12}\left[\frac{\mathrm{rad}}{\mathrm{s} \cdot \mathrm{K}}\right] \\
\Delta \omega_{I} & \approx-2.49 \cdot 10^{11}\left[\frac{\mathrm{rad}}{\mathrm{s} \cdot \mathrm{mA}}\right]=-7.96 \cdot 10^{12}\left[\frac{\mathrm{rad}}{\mathrm{s} \cdot I_{t h}}\right]
\end{aligned}
$$



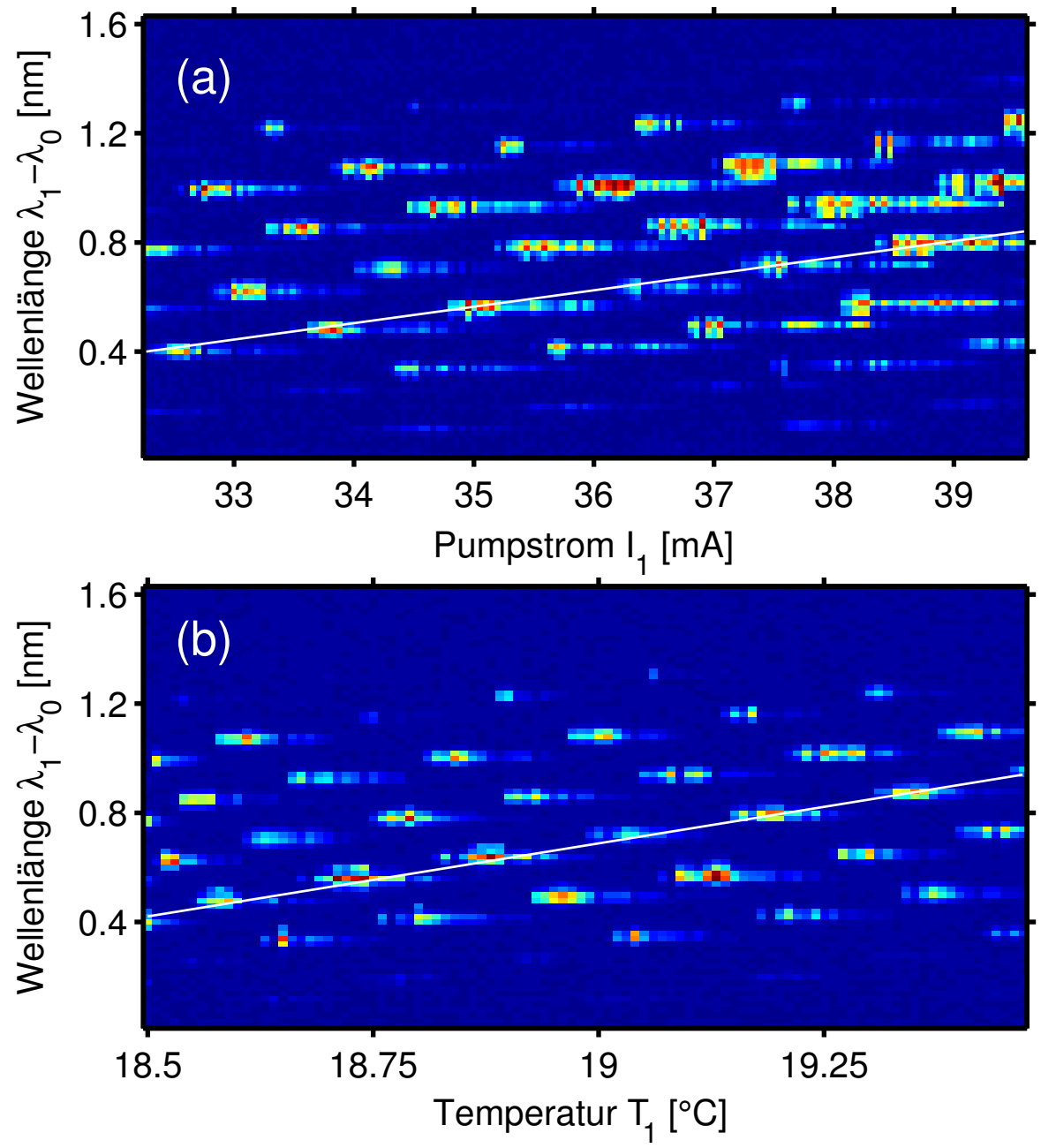

Abbildung 6.1: Bestimmung der Wellenlängenabhängigkeit von der Temperatur und dem Pumpstrom. 


\subsection{Numerisches Synchronisationschema}

Um mehrere Moden zu berücksichtigen habe ich die in Abschnitt 3.4 in den Gleichungen (3.11) und (3.12) beschriebene Erweiterung benutzt. Da davon auszugehen ist, dass die spektralen Modenabstände im Bereich von ca. 100-200GHz liegen, ist es plausibel anzunehmen, dass jede Mode des treibenden Lasers maximal mit einer einzigen Mode des empfangenden Lasers koppelt2 2 . Die Differenzfrequenz $\omega_{m}-\hat{\omega}_{m}$ wird für alle Moden gleich angenommen.

Es wird eine unidirektionale Kopplung beider Lasergleichungssysteme verwendet:

$$
\begin{aligned}
\frac{\mathrm{d}}{\mathrm{d} t} E_{(m)}(t)= & {\left[\frac{i \alpha}{2} G\left(N, \omega_{m}\right)+\frac{1}{2}\left(G\left(N, \omega_{m}\right)-\frac{1}{\tau_{p}}\right)\right] E_{(m)}(t) } \\
& +\kappa E_{(m)}(t-\tau) e^{-i \omega_{m} \tau} \\
\frac{\mathrm{d}}{\mathrm{d} t} N(t)= & p J_{t h}-\gamma N(t)-\sum_{m} G\left(N, \omega_{m}\right)\left|E_{(m)}(t)\right|^{2} \\
\frac{\mathrm{d}}{\mathrm{d} t} \hat{E}_{(m)}(t)= & {\left[\frac{i \alpha}{2} G\left(\hat{N}, \hat{\omega}_{m}\right)+\frac{1}{2}\left(G\left(\hat{N}, \hat{\omega}_{m}\right)-\frac{1}{\tau_{p}}\right)\right] \hat{E}_{(m)}(t) } \\
& +\hat{\kappa} \hat{E}_{(m)}(t-\tau) e^{-i \hat{\omega}_{m} \tau}+\rho E_{(m)}\left(t-\tau_{c}\right) e^{-i \omega_{m} \tau_{c}} e^{i\left(\omega_{m}-\hat{\omega}_{m}\right) t} \\
\frac{\mathrm{d}}{\mathrm{d} t} \hat{N}(t)= & \hat{p} J_{t h}-\gamma \hat{N}(t)-\sum_{m} G\left(\hat{N}, \hat{\omega}_{m}\right)\left|\hat{E}_{(m)}(t)\right|^{2}
\end{aligned}
$$

Die Variablen des empfangenden Systems sind mit dem Dachsymbol versehen. Als Kopplung wird dem Feld des Empfangssystems $\hat{E}_{(m)}(t)$ in Gleichung (6.8) das mit der Kopplungsstärke $\rho$ skalierte und um die Kopplungszeit $\tau_{c}$ verzögerte Feld $\rho E_{(m)}\left(t-\tau_{c}\right) e^{-i \omega_{m} \tau_{c}} e^{i\left(\omega_{m}-\hat{\omega}_{m}\right) t}$ des treibenden Lasers hinzuaddiert. Es handelt sich demnach um eine kohärente Kopplung beider Laser. Die Gesamtintensitäten sind die addierten Intensitäten der einzelnen Moden

$$
\begin{aligned}
|E(t)|^{2} & =\sum_{m}\left|E_{(m)}(t)\right|^{2} \\
|\hat{E}(t)|^{2} & =\sum_{m}\left|\hat{E}_{(m)}(t)\right|^{2} .
\end{aligned}
$$

Mathematisch gesehen ist in diesem System identische Synchronisation erreichbar,

\footnotetext{
${ }^{2}$ Diese Annahme wird auch im multimodalen Lang-Kobayashi-Modell verwendet.
} 
wenn alle Systemparameter identisch sind und folgende Identitäten

$$
\begin{aligned}
\hat{E}_{(m)}(t) & =E_{(m)}\left(t-\tau_{c}+\tau\right) \\
\hat{N}(t) & =N\left(t-\tau_{c}+\tau\right) \\
\hat{\kappa} & =\kappa-\rho \\
\hat{\omega}_{m} & =\omega_{m}
\end{aligned}
$$

gelten. Durch Einsetzen in (6.8) und (6.9) lässt sich dies leicht verifizieren. Dass diese Synchronisation auch stabil gegenüber leichten Parametervariationen ist, konnten AHLERs et al. für den monomodalen Fall anhand von numerischen Rechnungen zeigen. Gleichung (6.14) zeigt auch eine Möglichkeit dieses Schema im Experiment zu vereinfachen: Gilt $\rho=\kappa$, so kann auf einen externen Resonator im zweiten System verzichtet werden, da dann $\hat{\kappa}=0$ ist.

\section{Antizipierte und retardierte Synchronisation}

Vernachlässigt man in den Gleichung (6.12) und (6.13) die Kopplungsverzögerung $\tau_{c}$ so ergibt sich $\hat{E}_{(m)}(t)=E_{(m)}(t+\tau)$ bzw. $\hat{N}(t)=N(t+\tau)$. Die Dynamik des empfangenden Systems eilt also dem treibendem System in gewissem Sinne Vorraus. Diese s.g. antizipierte Synchronisation gilt solange $\tau_{c}<\tau$ und wurde von MASOLLER 2001 [45] numerisch anhand der obigen Gleichungen beschrieben. AHLERs et al. zeigte in seiner Veröffentlichung 1998 [5] lediglich die Synchronisation mit $\tau_{c}>\tau$, wodurch hier retardierte Synchronisation auftrat. Experimentell konnte die antizipierte Synchronisation erstmals von SivAPRAKASAm et al. 2001 [64] beobachtet werden.

Diese Veröffentlichung erzeugte auch in einigen populärwissenschaftlichen Zeitungen einiges Aufsehen, da durch die Autoren auch in weiteren Veröffentlichungen suggeriert wurde, dass das empfangende System die Dynamik des treibende System in gewissem Sinne vorhersagt. Diese Darstellung ist so aber falsch, wenn man sich den Informationsfluss genauer vor Augen hält. Das empfangende Lasersystem erhält nämlich das Feld des treibenden Laser zum Zeitpunkt $t$ genau um die Kopplungszeit $\tau_{c}$ später. Der treibende Laser selber erhält dieses Feld genau um die Umlaufzeit des externen Resonators $\tau$ später. Wenn nun $\tau_{c}<\tau$, so wird der empfangende Laser genau $\tau-\tau_{c}$ früher mit dem Feld gekoppelt als der treibende Laser selber. Mit dieser Erläuterung wird auch klar, dass dieses Voreilen des Empfangssystems maximal der Umlaufdauer des externen Resonators im treibenden Systems betragen kann (wenn $\tau_{c}=0$ ). 


\section{Kopplungsverzögerung im Experiment}

Betrachtet man die im Experiment ermittelte Verzögerungszeit der Reaktion des Lasers im Vergleich zu den Signallaufzeiten gemäß des verwendeten Aufbaus (Abb. 5.11), so ergibt sich laut Gleichung (6.12) $\tau_{c}-\tau=5.5 \mathrm{~ns}$. Die Umlaufdauer im externen Resonator des treibenden Systems ist $\tau=2 \cdot 33.5 \mathrm{~cm} / c \approx 2.23 \mathrm{~ns}$. Für die Kopplungsverzögerung ergebe sich dann $\tau_{c}=5.5 \mathrm{~ns}+\tau=7.73 \mathrm{~ns}$ oder einer Entfernung von ca. $2.32 \mathrm{~m}$. Dies ist bedeutend länger als die real im Experiment verwendete Kopplungsentfernung von nur ca. $1.58 \mathrm{~m}$. Mit einer Kopplungsverzögerung von $\tau_{c}$ ergibt sich eine Kopplungsentfernung von ca. $1.65 \mathrm{~cm}$. Dies kommt der realen Entfernung sehr nahe. Also entgegen der Lösung aus Gleichung (6.12) tritt im Experiment offensichtlich $\hat{E}(t)=E\left(t-\tau_{c}\right)$ auf. Eine andere Kopplungsverzögerung war in meinem Aufbau bisher nicht festzustellen, sie scheint unabhängig gegenüber Parameteränderungen zu sein. In Simulationen von LOCQUET et al. 2001 [4] konnte gezeigt werden, dass mit diesem Synchronisationsschema auch diese Synchronisationsverzögerung möglich ist.

\subsection{Synchronisation und Antisynchronisation der LFF- Dynamik}

Zur Lösung des Gleichungssystems habe ich die Prediktor-Korrektor-Methode der häufig verwendeten Runge-Kutta-Methode vorgezogen, da erstere eine deutlich höhere Rechengeschwindigkeit aufwies. Im Gegensatz zur experimentellen Realisierung der Synchronisation sind zwar die Parameter im numerischen Fall komplett kontrollierbar. Leider führen viele Parameterkombinationen zu numerisch instabilen Systemen. Dies gilt u. a. auch für Bereiche des Pumpstromes und der Einkoppelstärke, die im Experiment noch problemlos erreichbar sind. Die von mir verwendeten numerischen Parameter sind in Anhang D zu entnehmen.

Dieses hier verwendete Modell kann aufgrund der vielen in der Herleitung vorkommenden Näherungen und Vereinfachungen, als auch wegen der nicht exakt bekannten Laserparameter keine quantitativ vergleichbaren Ergebnisse liefern. Die qualitativen Vergleiche sind jedoch, vor allem unter Berücksichtigung der Komplexität der Dynamik, erstaunlich gut. Mit der Annahme, dass diesen qualitativ ähnlichen Ergebnissen in der numerischen Simulation und dem Experiment, dieselben Mechanismen zugrundeliegen, ist es auch möglich, die im Experiment von mir nicht beobachtbare Dynamik der extrem schnellen Pulsdynamik anhand der numerischen Simulation zu studieren (siehe Abschnitt 6.7). 

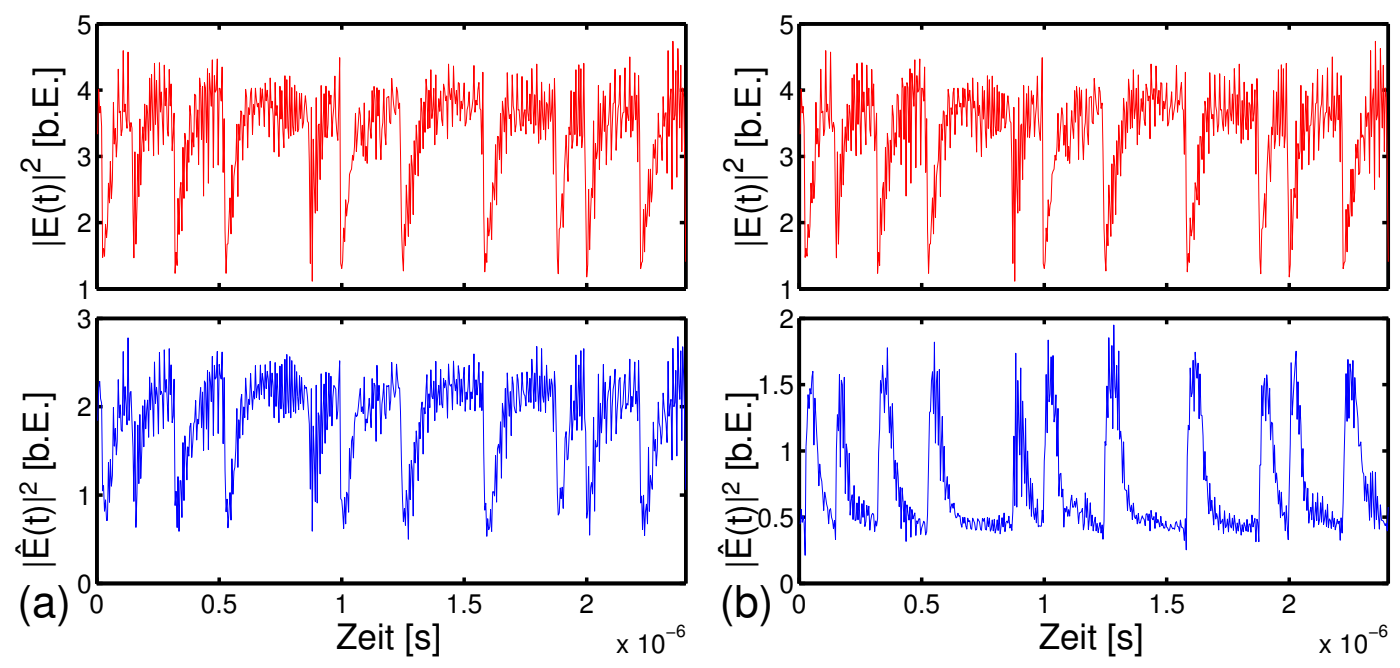

Abbildung 6.2: Numerischen Synchronisation (a) und Antisynchronisation (b) der LFF-Dynamik.

In Abbildung 6.2 ist beispielhaft die numerische Synchronisation und Antisynchronisation der LFF-Dynamik dargestellt. Verwendet wurden für die Simulation Gleichungen (6.6)-(6.14) . Für alle Betrachtungen der numerisch berechneten Intensitäten in diesem Kapitel wurde eine Tiefpassfilterung mit einer Grenzfrequenz von $1 \mathrm{GHz}$ durchgeführt, um einen Vergleich mit den experimentell ermittelten Intensitäten zu ermöglichen (mit Ausnahme des Abschnitts 6.7). Nach der Durchführung mehrerer Simulationen mit diesen Multimode-LK-Gleichungen stellte sich heraus, dass die Anzahl der Moden nur einen geringen Einfluss auf das qualitative Aussehen der Gesamtintensität zu haben scheint. Zur Verringerung des Rechenaufwands habe ich deshalb die Modenanzahl auf drei beschränkt.

\subsubsection{Parameterbereiche der Synchronisation und Antisynchronisa- tion}

In der Abbildung 6.3 ist der Synchronisationsindex systematisch für einen Bereich des normierten Pumpstroms $\hat{p}$ des Empfangssystems und der Frequenzdifferenz $\Delta \omega=$ $\omega-\hat{\omega}$ dargestellt. Das Empfangssystem wurde hierbei ohne externen Resonator simuliert $(\hat{\kappa}=0)$. Sehr gut lassen sich die Gebiete der Synchronisation und der Antisynchronisation erkennen. Sehr auffällig ist, dass die Antisynchronisation für den Parameterbereich $\hat{p} \approx 1.01$ und unterhalb von $\Delta \omega \approx-2 \cdot 10^{11}$ relativ abrupt verschwindet. Abgesehen von diesem Bereich zeigt sich in dem aufgetragenen Parameterbereich keine weiteren Gebiete mit einem Synchronisationindex von näherungsweise Null. Auffällig 


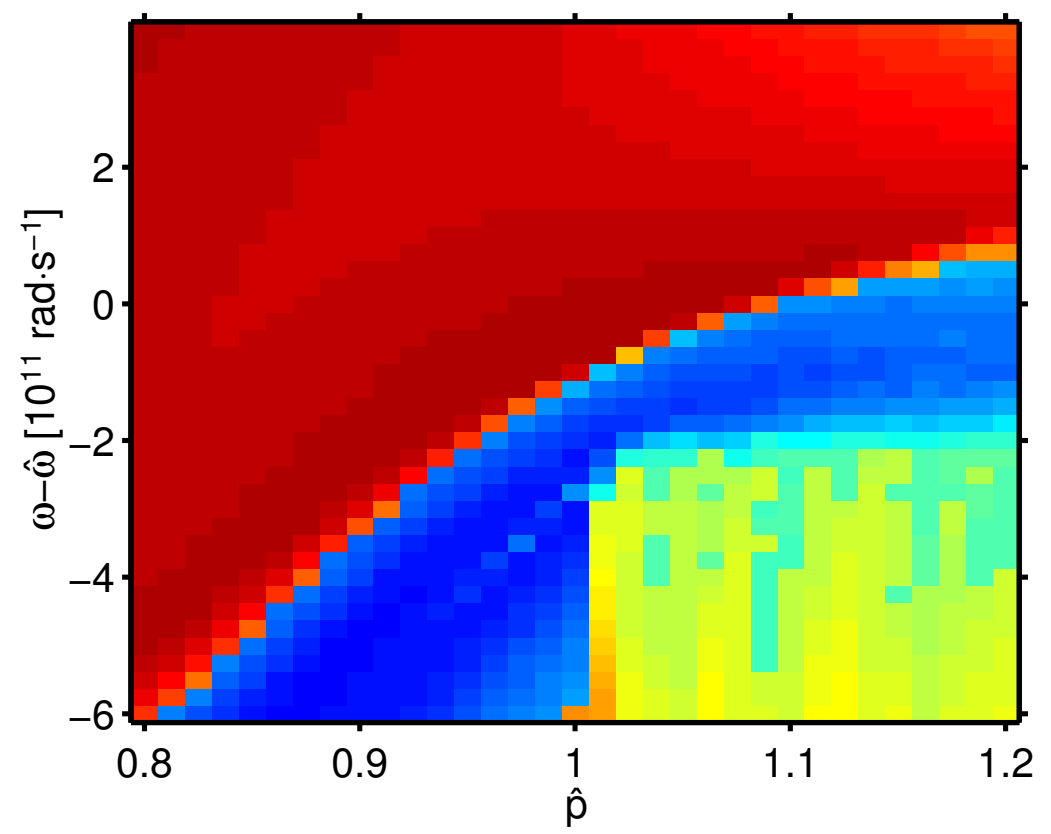

Abbildung 6.3: Synchronisationsindex farbkodiert aufgetragen über der Frequenzverstimmung $\Delta \omega$ und dem normierten Pumpstrom $\hat{p}$ des Empfangssystems.

ist auch, das die Grenzlinie zwischen Synchronisationsgebiet und Antisynchronisationsgebiet keinen linearen Verlauf sondern einen charakteristischen Bogen darstellt. Außerhalb des dargestellten Bereiches nehmen die numerischen Probleme stark zu (die Gleichungen divergieren meistens), weswegen ich mich auf diesen Bereich beschränkt habe.

Um diese Ergebnisse mit den experimentellen Messungen vergleichen zu können, ist es sinnvoll die hier aufgetragenen Parameter $\Delta \omega, \hat{p}$ gemäß der Frequenzabhängigkeit aus Gleichung (6.3) in eine numerische Temperatur $T_{2}^{(n)}$ und einen numerischen Pumpstrom $I_{2}^{(n)}$ des Empfangssystems umzurechnen. In der Auftragung 6.4 w wurde dies mit den Daten der Rechnungen aus Abbildung 6.3 durchgeführt. Das experimentelle Pendant hierzu ist in Auftragung 6.4 $\mathrm{b}$ zu sehen, dies ist ein Ausschnitt aus Abbildung 5.13. Da die numerische Temperatur bis auf eine Konstante bestimmt ist, wurde lediglich die Temperaturdifferenz $\Delta T_{1}^{(n)}$ dargestellt. Für die Darstellung der experimentellen Daten wurde zur besseren Vergleichbarkeit ebenfalls eine Differenztemperatur $\Delta T_{1}$ mit einer geeigneten Referenztemperatur verwendet. Qualitativ ist eine gute Übereinstimmung festzustellen. Speziell die Reihenfolge des Auftretens der Synchronisations- und Antisynchronisationsbereiche und der Verlauf der Trennlinie zwischen beiden Bereichen werden von der Numerik gut wiedergegeben. Angemerkt sei hier noch, dass in den weißen Bereichen in Auftragung 6.4 a, aufgrund der bereits 

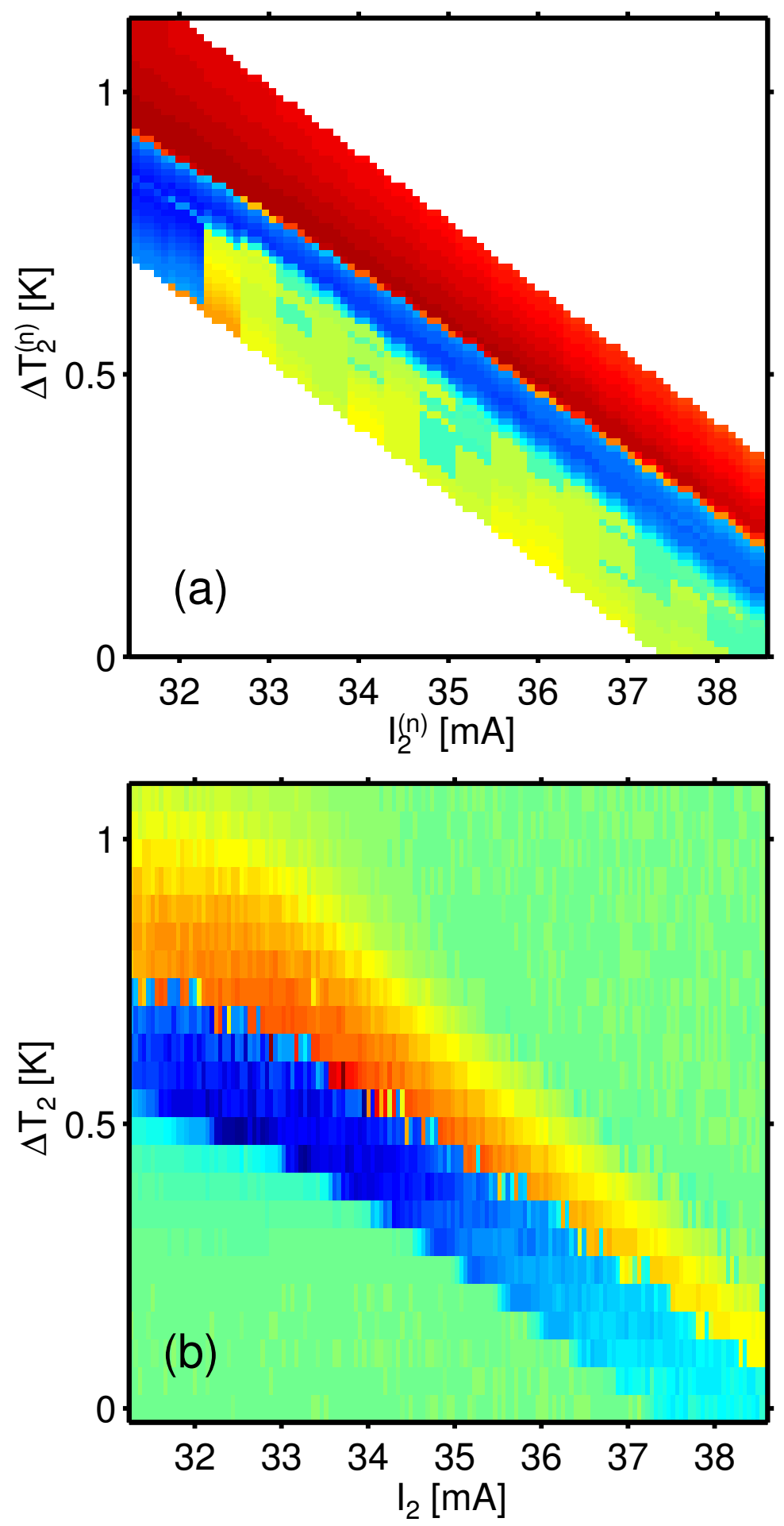

Abbildung 6.4: Vergleich der numerischen Ergebnisse (a) mit den experimentellen Messungen (b). 
erwähnten numerischen Instabilitäten keine Rechenergebnisse vorliegen.

Für den Fall, dass der Pumpstromparameter $p$ des treibenden Systems variiert wird ergibt sich die in Abbildung 6.5 dargestellte Auftragung. Der Pumpstrombereich beginnt hier erst bei $p=1.02$, da das treibende System unterhalb dieses Wertes keine LFF-Dynamik zeigt. Bei den beiden vertikalen rötlichen Linien in der Auftragung fehlt diese Dynamik ebenfalls, wodurch der Synchronisationsindex hier keinen Aussagewert besitzt. In den übrigen Bereichen erkennt man hingegen sehr gut den Übergang von Synchronisation (rot) zur Antisynchronisation (blau) sowie nicht synchronisierte Bereiche (grün). Der Übergang ist im wesentlichen von der Frequenzdifferenz $\Delta \omega$ abhängig.

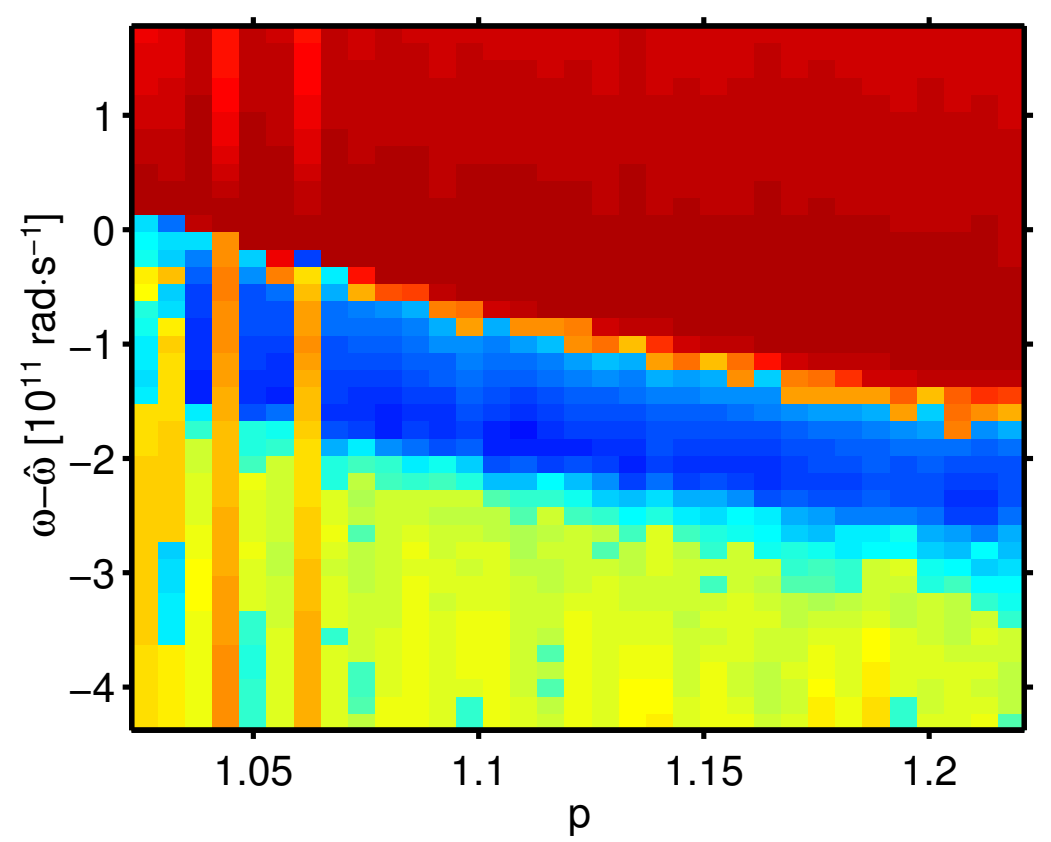

Abbildung 6.5: Synchronisationsindex farbkodiert aufgetragen über der Frequenzverstimmung $\Delta \omega$ und dem normierten Pumpstrom $p$ des treibenden Systems.

Zwar zeigen beiden Auftragungen 6.3 und 6.5] in Bezug auf die Frequenzdifferenz $\Delta \omega$ in derselben Reihenfolge Synchronisation und Antisynchronisation. Zum Vergleich mit den experimentellen Ergebnissen in Abbildung 6.6 dreht sich in diesem Fall die Reihenfolge jedoch um, da die numerische Temperatur des treibenden Systems $T_{1}^{(n)}$ invertiert zu der des empfangenden Systems in Gleichung (6.3) eingeht. Dies wird sehr gut durch die experimentellen Ergebnisse bestätigt, wie die Auftragung 6.6b zeigt (dies ist ein Ausschnitt aus Abbildung (5.14). 

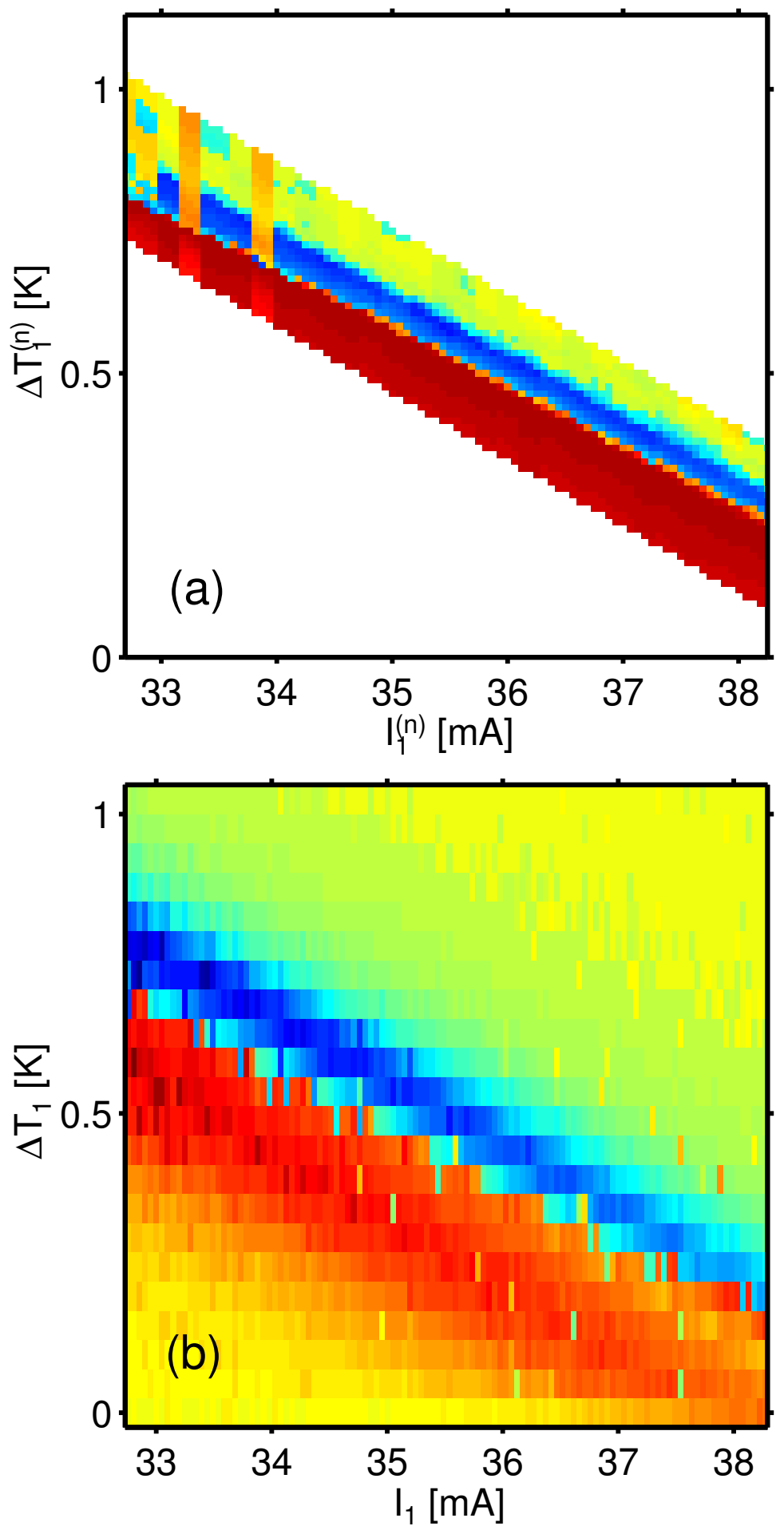

Abbildung 6.6: Vergleich der numerischen Ergebnisse (a) mit den experimentellen Messungen (b). 

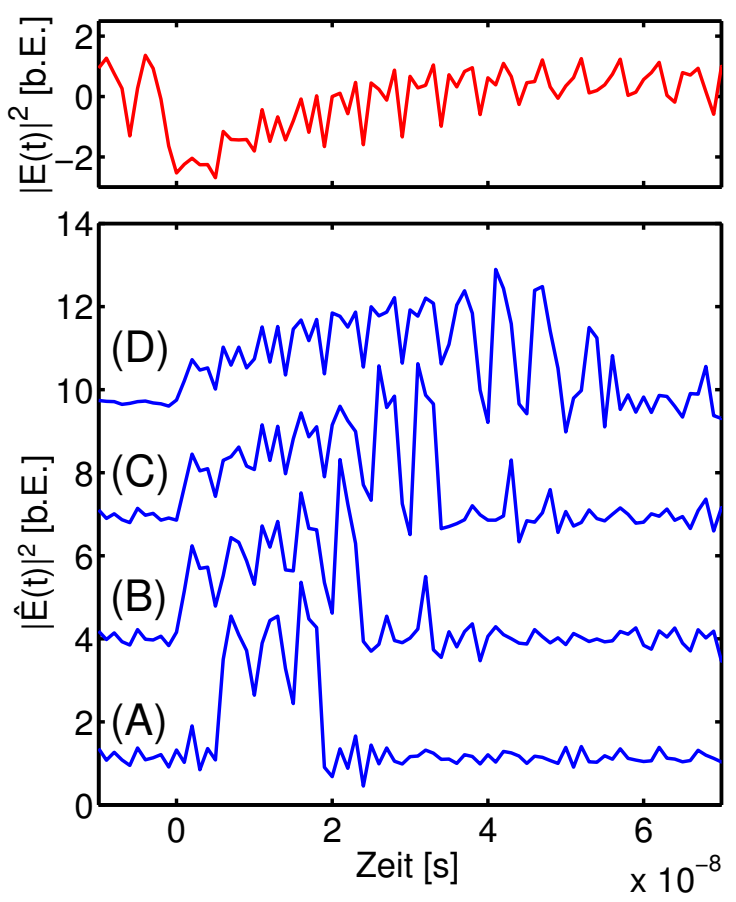
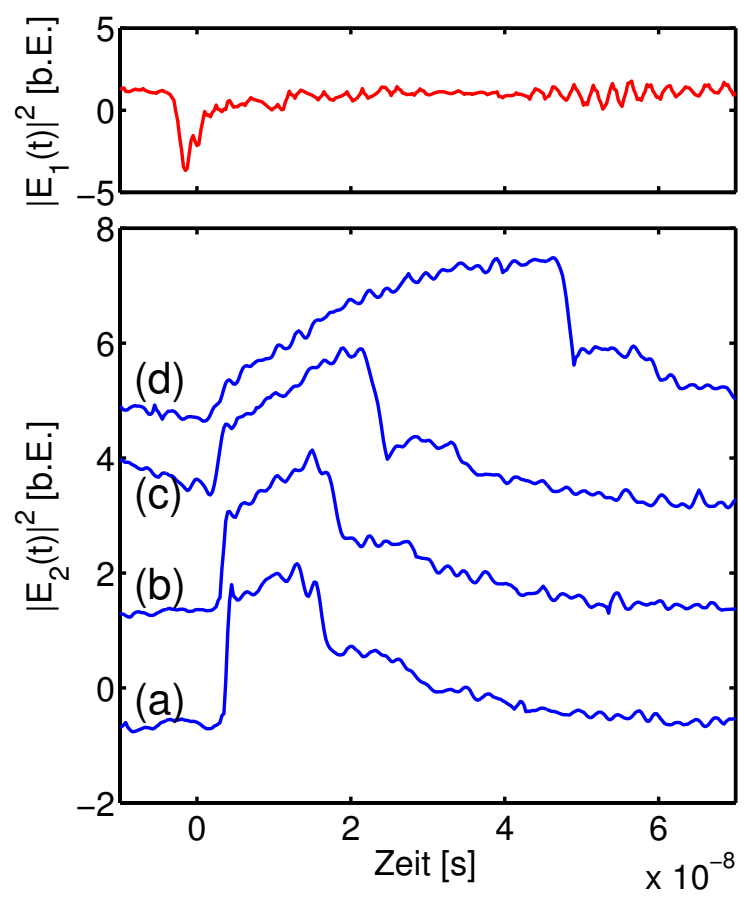

Abbildung 6.7: Links: Numerisch berechnete Zeitreihen der Laserintensität für verschiedene Parameter $I_{2}^{(n)}$ im Übergangsbereich zwischen Synchronisation und Antisynchronisation: (A) $I_{2}^{(n)}=34.16 \mathrm{~mA}$, (B) $I_{2}^{(n)}=34.32 \mathrm{~mA},(\mathrm{C})$ $I_{2}^{(n)}=34.48 \mathrm{~mA}$, (D) $I_{2}^{(n)}=34.64 \mathrm{~mA}$. Rechts: Korrespondierende experimentell gemessene Intensitätskurven (a), (b), (c) und (d) aus Abbildung 5.11 


\subsection{2 Übergang zwischen Synchronisation und Antisynchronisation}

In die linken Auftragung in Abbildung 6.7 ist der Übergang von Antisynchronisation zu Synchronisation anhand von Intensitätskurven des treibenden und des empfangenden Laser dargestellt. Um einen Vergleich zu der korrespondierenden experimentellen Auftragung (Abb. 6.7 rechts) zu erzielen, habe ich für die Parameter $\hat{p}, \Delta \omega$ die Werte des numerischen Pumpstroms angegeben 3 .

Die Intensitätskurven der numerischen Rechnung sind mit einer Grenzfrequenz von $1 \mathrm{GHz}$ tiefpassgefiltert. Es ist klar erkennbar, dass die experimentell ermittelten Intensitäten deutlich stärker tiefpassgefiltert wurden (die Detektoren haben in etwa eine Bandbreite von $200 \mathrm{MHz}$ ). Die Reaktion auf das Kopplungssignal stimmt ansonsten sehr gut überein. Zum Zeitpunkt des Intensitätseinbruchs des treibenden Lasers (rote Kurve) steigt sowohl im Experiment als auch in der numerischen Simulation die Intensität sprunghaft an. Mit einer vom Pumpstrom abhängigen Verzögerung bricht die Intensität wieder zusammen. Der Mechanismus des Übergangs von Antisynchronisation zu Synchronisation wird also von dem Modell qualitativ gut beschrieben.

Für den Fall mit externem Resonator im Empfangssystem sind die Intensitätskurven des Übergangs von Antisynchronisation zu Synchronisation in Abbildung 6.8 dargestellt. Der Übergang verläuft ähnlich wie im Experiment, jedoch zeigen sich hier deutliche Unterschiede im Zeitverlauf sowie bei dem benötigten Pumpstrom. Das Problem ist hierbei vor allem die sehr große Empfindlichkeit von den Parametern. Dieses Problem zeigte sich ebenfalls in der experimentellen Realisierung. Auch ließ sich die Größe der externen Resonatoren im Experiment nicht ausreichend gut justierent Quantitativ vergleichbare numerische Resultate sind aus diesem Grund nicht zu erwarten. Auf systematische Parametervariationen wurde deshalb für den Fall optischer Rückkopplung im Empfangssystem sowohl experimentell als auch numerisch verzichtet.

\subsection{Synchronisation und Antisynchronisation der LFJ- Dynamik}

Zur Simulation der LFJ-Dynamik habe ich das in Abschnitt 3.5 beschriebene Modell verwendet. Dieses Modell wurde gemäß Abschnitt 3.4 auf mehrere Moden erweitert und

\footnotetext{
${ }^{3}$ Hierbei wurde ein Laserschwelle von $I_{t h}=32 \mathrm{~mA}$ angenommen. Die numerischen Parameter lauten: $p=1.08$, (a) $\hat{p}=1.0675, \Delta \omega=-1.6 \cdot 10^{11} \mathrm{rad} \cdot \mathrm{s}^{-1}$, (b) $\hat{p}=1.0725, \Delta \omega=-1.2 \cdot 10^{11} \mathrm{rad} \cdot \mathrm{s}^{-1}$, (c) $\hat{p}=1.0775, \Delta \omega=-8 \cdot 10^{10} \mathrm{rad} \cdot \mathrm{s}^{-1}$, (d) $\hat{p}=1.0825, \Delta \omega=-4 \cdot 10^{10} \mathrm{rad} \cdot \mathrm{s}^{-1}$.

${ }^{4}$ Die Resonatoren besaßen in der numerischen Simulation eine identische Länge, dies war im Experiment mit den zur Verfügung stehenden Mitteln nicht mit der benötigten Genauigkeit realisierbar.
} 

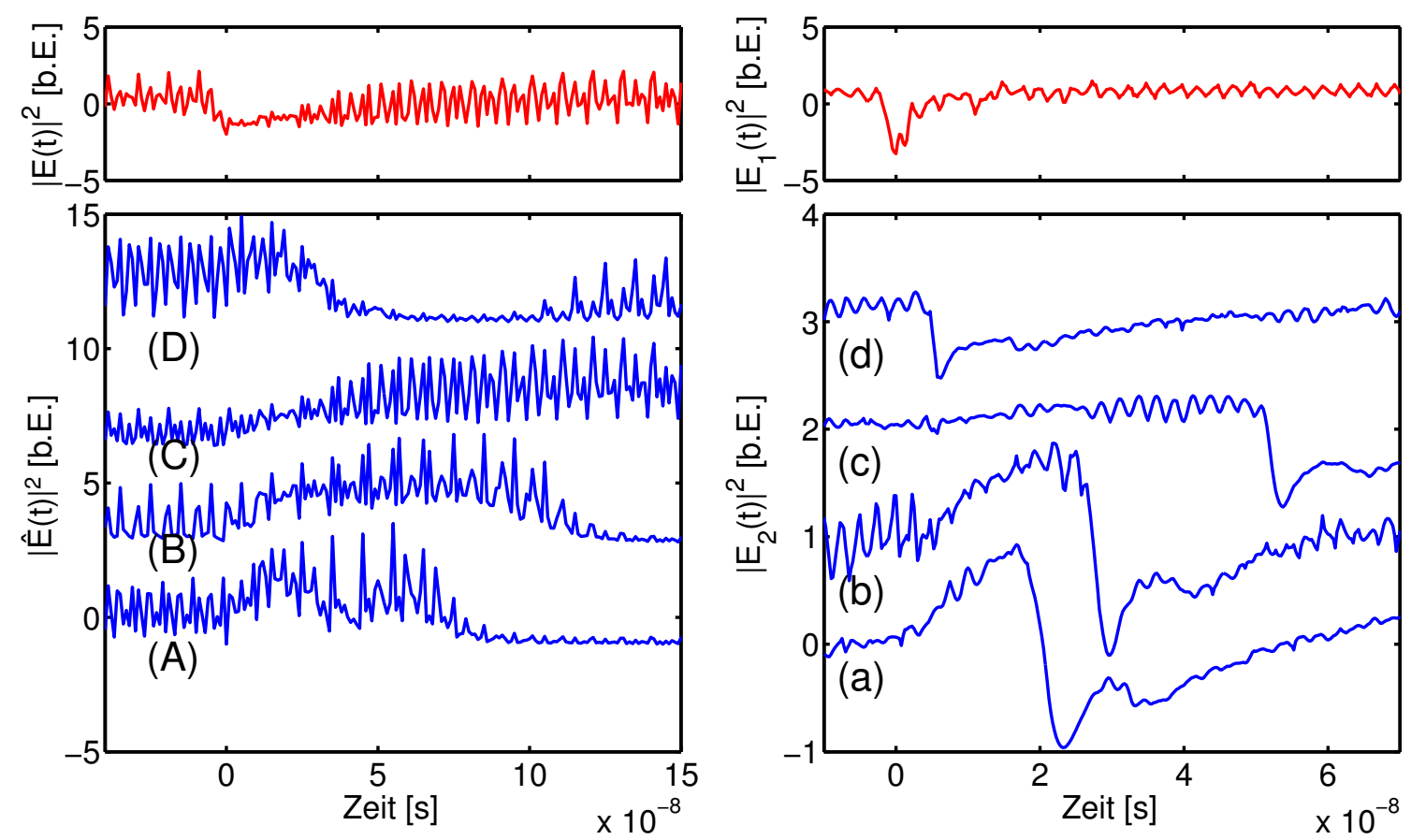

Abbildung 6.8: Links: Numerisch berechnete Zeitreihen der Laserintensität für verschiedene Parameter $I_{2}^{(n)}$ im Übergangsbereich zwischen Synchronisation und Antisynchronisation mit externem Resonator im Empfangssystem: (A) $I_{2}^{(n)}=30.72 \mathrm{~mA},(\mathrm{~B}) I_{2}^{(n)}=30.8 \mathrm{~mA}$, (C) $I_{2}^{(n)}=30.88 \mathrm{~mA},(\mathrm{D})$ $I_{2}^{(n)}=30.94 \mathrm{~mA}$, (E) $I_{2}^{(n)}=31.02 \mathrm{~mA}$. Rechts: Korrespondierende experimentell gemessene Intensitätskurven (a),(b),(c),(d) und (e) aus Abbildung [5.10, 
mit einem normalem Multimode-LK-Modell gekoppelt. Die resultierenden Gleichungen sind:

$$
\begin{aligned}
\frac{\mathrm{d}}{\mathrm{d} t} E_{(m)}(t)= & {\left[\frac{i \alpha}{2} G\left(N, \omega_{m}\right)+\frac{1}{2}\left(G\left(N, \omega_{m}\right)-\frac{1}{\tau_{p}}\right)\right] E_{(m)}(t) } \\
& +\kappa A_{(m)}(t-\tau) e^{-i \omega_{m} \tau} \\
A_{(m)}(t)= & E_{(m)}(t)+\sum_{n=1}^{\infty}\left(r_{2} r_{3} e^{-i \omega_{m} \tau}\right)^{n} E_{(m)}(t-n \tau) \\
\frac{\mathrm{d}}{\mathrm{d} t} N(t)= & p J_{t h}-\gamma N(t)-\sum_{m} G\left(N, \omega_{m}\right)\left|E_{(m)}(t)\right|^{2} \\
\frac{\mathrm{d}}{\mathrm{d} t} \hat{E}_{(m)}(t)= & {\left[\frac{i \alpha}{2} G\left(\hat{N}, \hat{\omega}_{m}\right)+\frac{1}{2}\left(G\left(\hat{N}, \hat{\omega}_{m}\right)-\frac{1}{\tau_{p}}\right)\right] \hat{E}_{(m)}(t) } \\
& +\rho A_{(m)}\left(t-\tau_{c}\right) e^{-i \omega_{m} \tau_{c}} e^{i\left(\omega_{m}-\hat{\omega}_{m}\right) t} \\
\frac{\mathrm{d}}{\mathrm{d} t} \hat{N}(t)= & \hat{p} J_{t h}-\gamma \hat{N}(t)-\sum_{m} G\left(\hat{N}, \hat{\omega}_{m}\right)\left|\hat{E}_{(m)}(t)\right|^{2} \\
|A(t)|^{2}= & \sum_{m}\left|A_{(m)}(t)\right|^{2}
\end{aligned}
$$
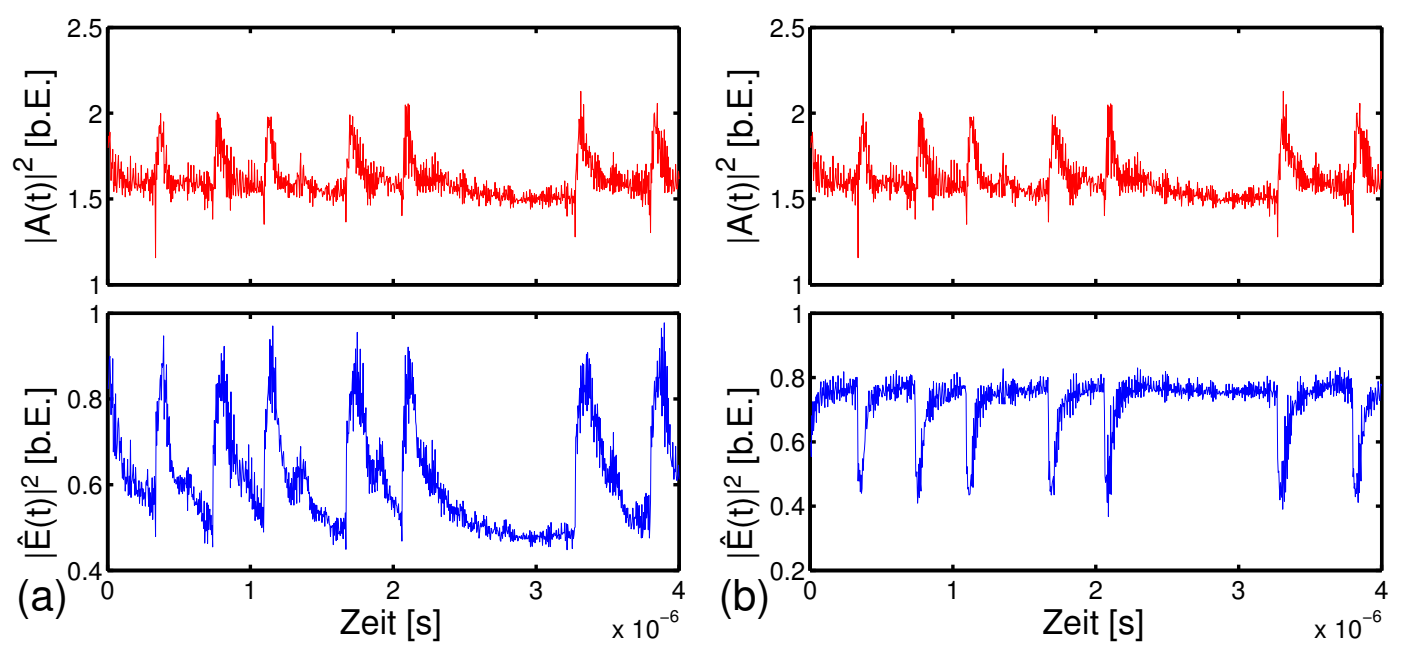

Abbildung 6.9: Numerische Simulation der Synchronisation (a) und der Antisynchronisation (b) der LFJ-Dynamik.

In Abbildung 6.9] sind die simulierten Intensitätssignale des treibenden Systems $|A(t)|^{2}$ (rechte Laserfacette gemäß Abbildung 3.8) und des empfangenden Systems $|\hat{E}(t)|^{2}$ für Synchronisation und Antisynchronisation dargestellt. Zur Vergleichbarkeit mit den experimentellen Intensitätskurven wurde auch hier eine Tiefpassfilterung durchgeführt. 
Die Ergebnisse der systematischen Variation der Parameter $\hat{p}$ und $\Delta \omega$ zeigt Abbildung 6.10. Hier ist wieder der Synchronisationsindex farbkodiert aufgetragen. Es lassen sich gut die Bereiche der Synchronisation und Antisynchronisation erkennen. Die Darstellung ist der Abbildung 6.3 sehr ähnlich, jedoch sind hier die Synchronisationsgebiete genau vertauscht. Das Synchronisationgebiet in dieser Auftragung ist in der Abbildung 6.3 das Antisynchronisationgebiet und umgekehrt.

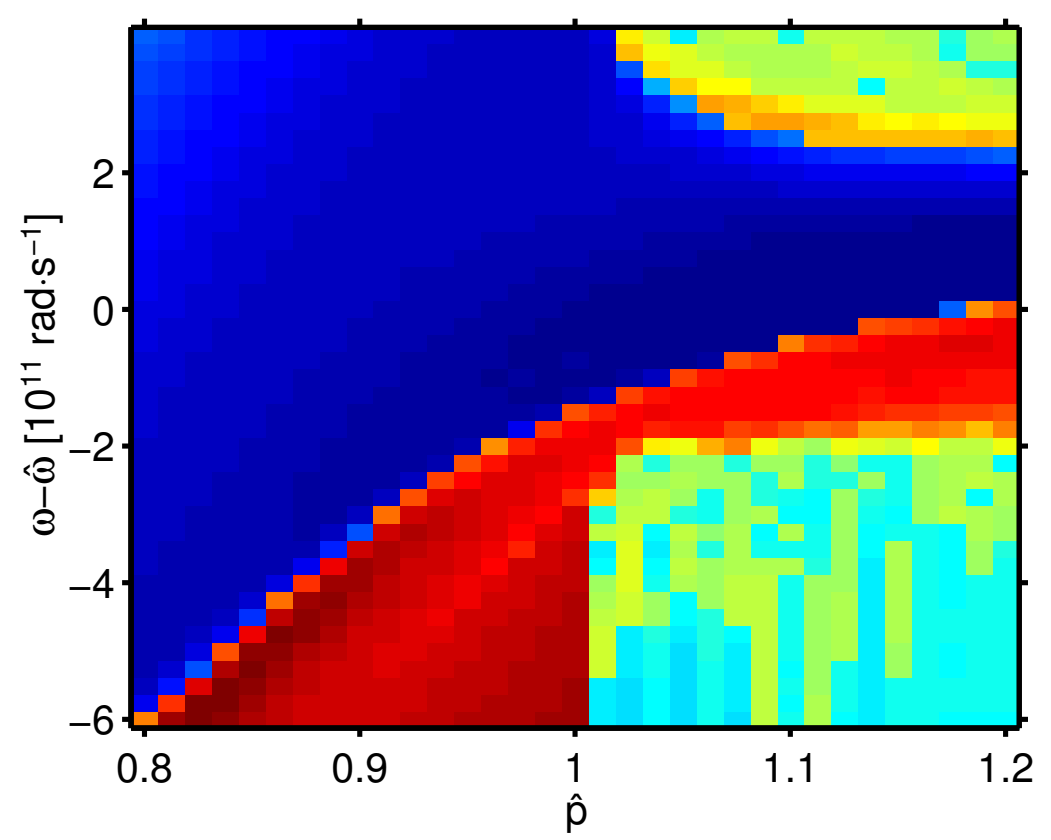

Abbildung 6.10: Synchronisationsindex farbkodiert aufgetragen über der Frequenzverstimmung $\Delta \omega$ und dem normierten Pumpstrom $\hat{p}$ des Empfangssystems.

Dies lässt sich relativ leicht verstehen. Um eine Vergleichbarkeit dieses numerischen Ergebnisses mit den experimentellen zu ermöglichen, wurde der Synchronisationsindex für diese Auftragung anhand der tiefpassgefilterten Intensitäten des Resonatorfeldes $|A(t)|^{2}$ und des empfangenden Systems $|\hat{E}(t)|^{2}$ berechnet. Im Fall von LFJ-Dynamik im treibenden System ist die tiefpassgefilterte Resonatorfeldintensität antikorreliert zur tiefpassgefilterten Laserfeldintensität des treibenden Lasers, wie im Abschnitt [3.5] beschrieben wurde. Dies bedeutet, dass im Antisynchronisationgebiet in Abbildung 6.10 die tiefpassgefilterte Laserfeldintensität synchronisiert mit der tiefpassgefilterten Feldintensität des empfangenden Systems ist. Es ist daher sehr gut nachzuvollziehen, dass sich die Synchronisationsgebiete gegenüber Abbildung 6.3 vertauschen.

Für den Vergleich der numerischen Berechnungen aus Abbildung 6.10 wurden die Parameter wieder auf die numerische Temperatur $T_{2}^{(n)}$ und den numerischen Pumpstrom $I_{2}^{(n)}$ umgerechnet. In Abbildung 6.11] sind in Auftragung 6.11] diese so umgerech- 
neten Ergebnisse zu erkennen. Die korrespondierende experimentelle Messung zeigt Auftragung 6.11 b. Es sind recht deutliche Unterschiede auszumachen. Speziell im Synchronisationsbereich unterscheiden sich beide Auftragungen deutlich. Die Abfolge der Synchronisationsgebiete sowie auch der Verlauf der Trennlinie zwischen den Synchronisationsbereichen wird hingegen relativ gut wiedergegeben.

Abbildung 6.12 zeigt die Auftragung des farbkodierten Synchronisationsindexes über der Frequenzverstimmung $\Delta \omega$ und dem Pumpstrom $p$ des treibenden Systems. Die Umrechnung in $T_{1}^{(n)}$ und $I_{1}^{(n)}$ zeigt Abbildung 6.13k. Die dazugehörige experimentelle Auftragung ist in Abbildung [6.13) 5 zu sehen (Ausschnitt aus Abb. [5.26). Auch zeigen sich deutliche Unterschiede in den Auftragungen. Wieder ist der Synchronisationsbereich in der numerischen Berechnung deutlich unterrepräsentiert. Jedoch wird ebenfalls die Reihenfolge sowie der Verlauf der Trennlinie der Synchronisationsgebiete befriedigend wiedergegeben. Zu erwähnen ist hier noch der deutlich unterschiedliche Pumpstrombereich beider Auftragungen. Derart hohe Pumpströme zeigen in den numerischen Berechnungen keine LFJ-Dynamik mehr.

\subsection{Modensynchronisation}

Im Experiment konnte die Modendynamik beider Laser lediglich auf derselben Frequenz verglichen werden. Die numerischen Simulation erlaubt die Betrachtung aller Modenintensitäten. Abbildung 6.14 zeigt die Gesamtintensitäten und die drei Modenintensitäten des treibenden und des empfangenden Lasers. Nicht sehr überraschend sind alle Moden des treibenden Lasers synchron mit der jeweiligen Gesamtintensität. Jede treibende Mode ist mit ihrer korrespondierenden Mode im Empfangssystem synchron, jedoch sind nicht alle Modenintensitäten identisch. In diesen Beispielauftragungen zeigt jede Einzelmode jeden Intensitätseinbruch der Gesamtintensität. Im Vergleich zu den experimentellen Messungen in Abbildung 5.23 ist dies ein deutlicher Unterschied. Im Experiment zeigt die betrachtete Modenintensität nicht alle von der Gesamtintensität ausgeführten Intensitätseinbrüche.

In Abbildung 6.15 sind die Intensitäten in derselben Weise für den Fall der antisynchronisierten LFF-Dynamik aufgetragen. Die Situation ist hier dieselbe wie bei der Synchronisation. Für den treibenden Laser war dies zu erwarten, da sich deren Parameter gegenüber den Auftragungen in Abbildung 6.14 nicht geändert haben. Für den Empfangslaser zeigt sich auch hier, dass jede Einzelmode näherungsweise synchron

\footnotetext{
${ }^{5}$ Leider führten Triggerprobleme dazu, dass diese Auftragung nur in geringerer Auflösung gemessen werden konnte.
} 

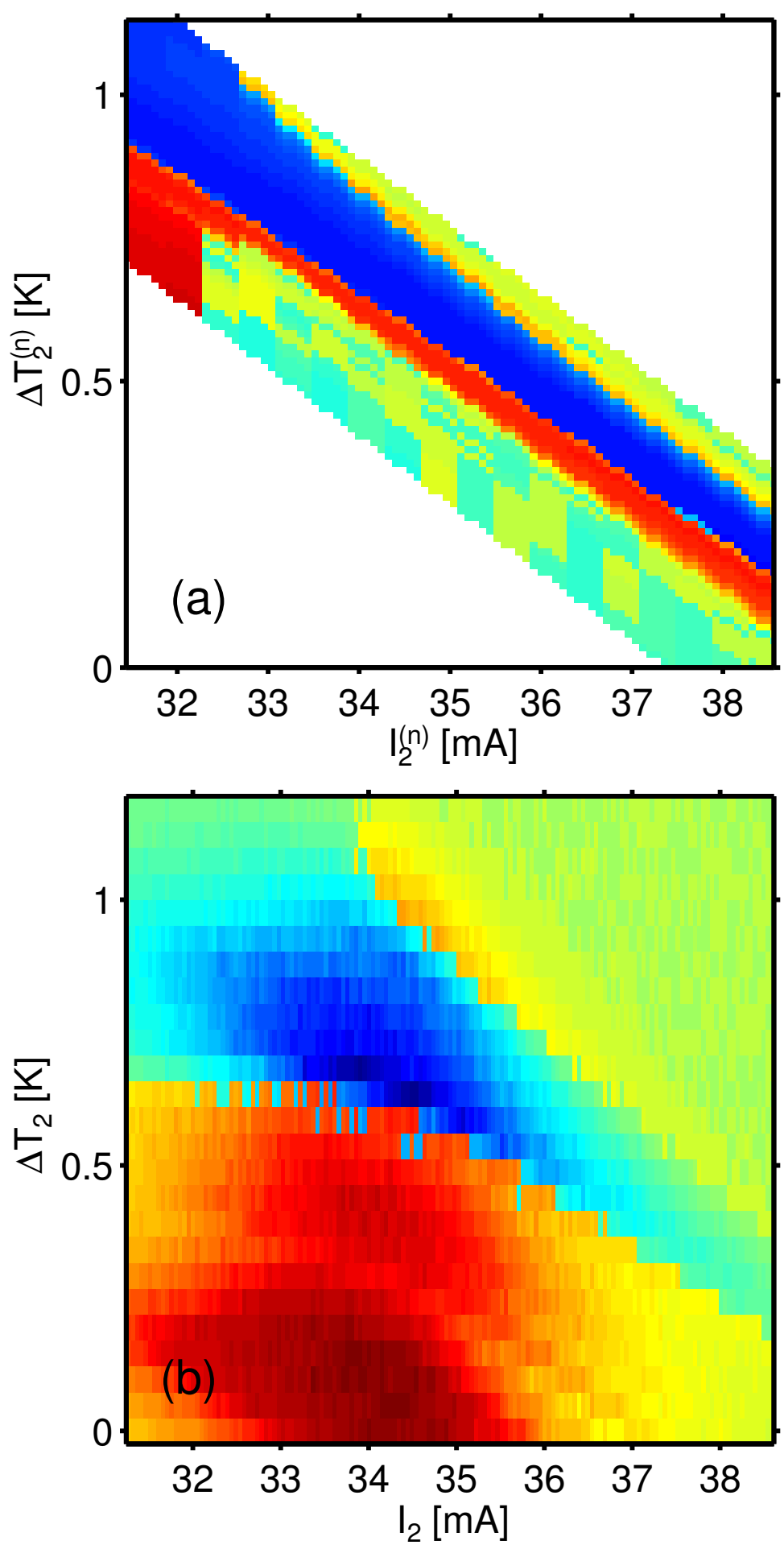

Abbildung 6.11: Vergleich der numerischen Ergebnisse (a) mit den experimentellen Messungen (b) bei der LFJ-Dynamik. 


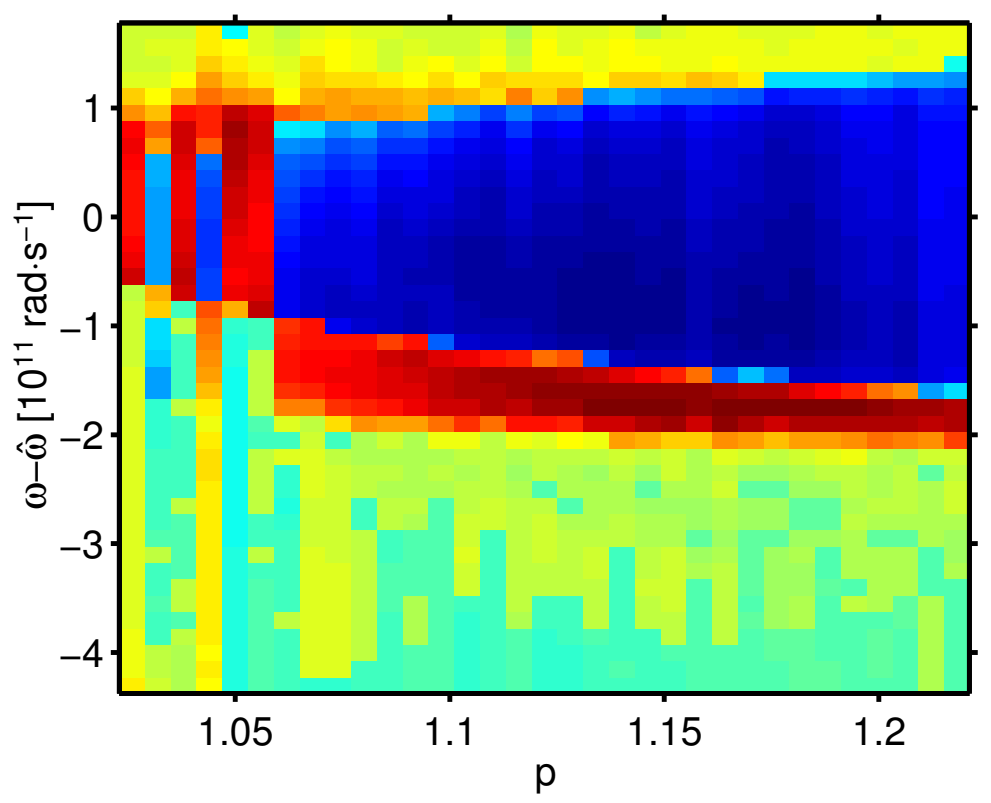

Abbildung 6.12: Synchronisationindex farbkodiert aufgetragen über der Frequenzverstimmung $\Delta \omega$ und dem normierten Pumpstrom $p$ des treibenden Systems.

mit der Gesamtintensitat als auch antisynchron zu ihrer korrespondierenden Mode im treibenden System verläuft.

Analysiert man die LFJ-Dynamik auf diese Weise, so gibt es einige überraschende Unterschiede im Verhalten des Systems. Die Situation wird bei dieser Dynamik noch etwas verkompliziert, da es für das treibende System zwei verschiedene Intensitäten für jede Mode gibt, die Intensität des Laserfeld und die des Resonatorfeldes wobei jedoch nur letzteres experimentell beobachtet werden konnte. In Abbildung 6.16] sind die Intensitätsauftragungen für den synchronisierten Fall, in Abbildung 6.17 für den antisynchronisierten Fall dargestellt. In beiden Abbildungen gehören immer drei untereinander dargestellte Auftragungen zusammen. Die Gesamtintensität ist in den Auftragungen (a), (b) und (c) jeweils für das Laserfeld des treibenden Lasers (grün), das Resonatorfeld des treibenden Lasers (rot) und das Feld des empfangenden Lasers (blau) dargestellt. Wie zuvor sind die Intensitäten der einzelnen Moden des Empfangslasers synchron zu ihren zugehörigen Moden des treibenden Lasers. Jedoch besitzt die zweite Mode eine komplett andere Dynamik als die erste und dritte. Die Intensität des Resonatorfeldes der zweiten Mode (Abb. 6.16h) zeigt im krassen Gegensatz zur Gesamtintensität und den anderen beiden Moden einzelne Intensitätseinbrüche ähnlich der LFF-Dynamik. Diese werden jedoch durch die umso stärkeren Intensitätssprünge der anderen beiden Moden kompensiert. Experimentell war dieser Fall nicht messbar. 

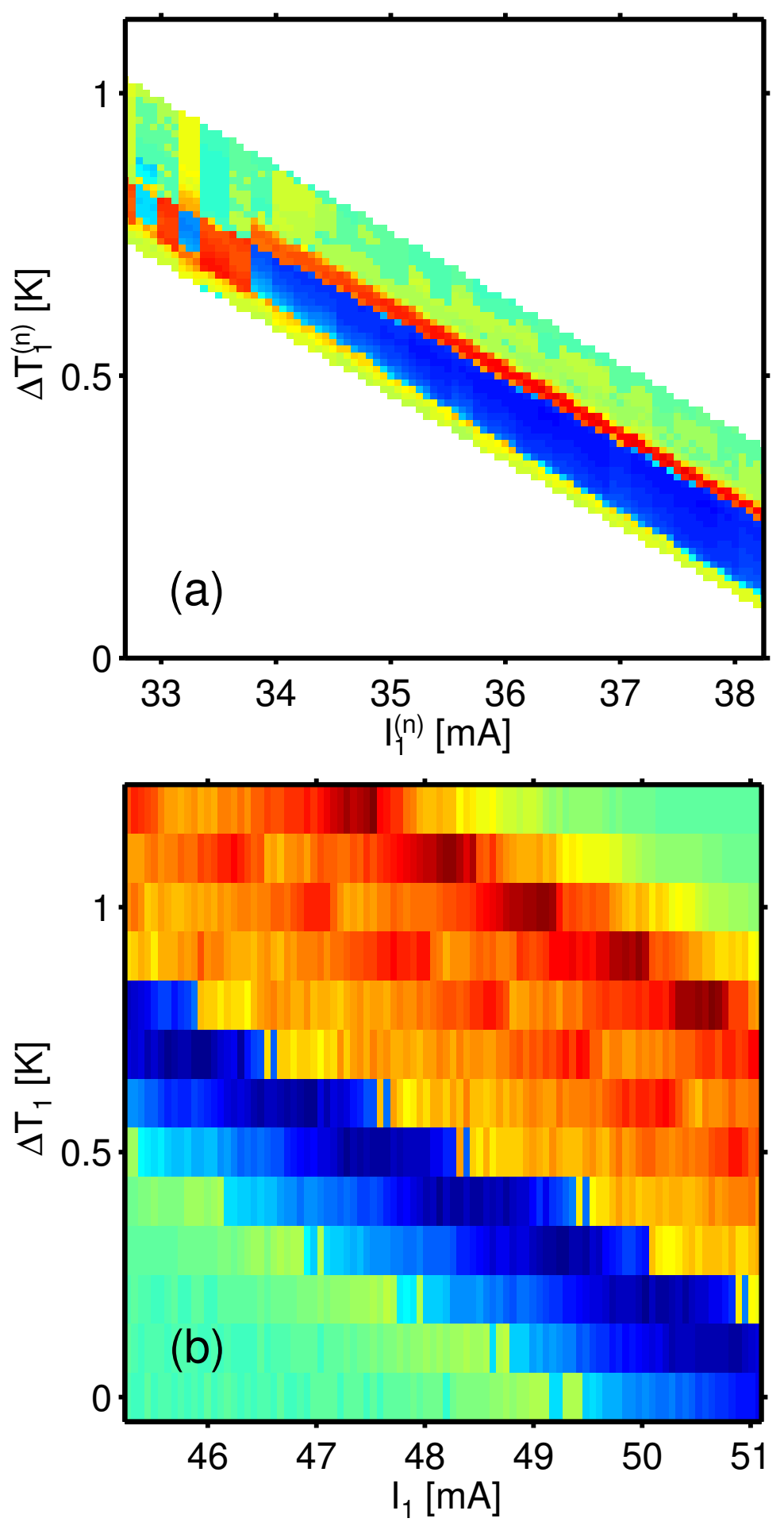

Abbildung 6.13: Vergleich der numerischen Ergebnisse (a) mit den experimentellen Messungen (b) bei der LFJ-Dynamik. 

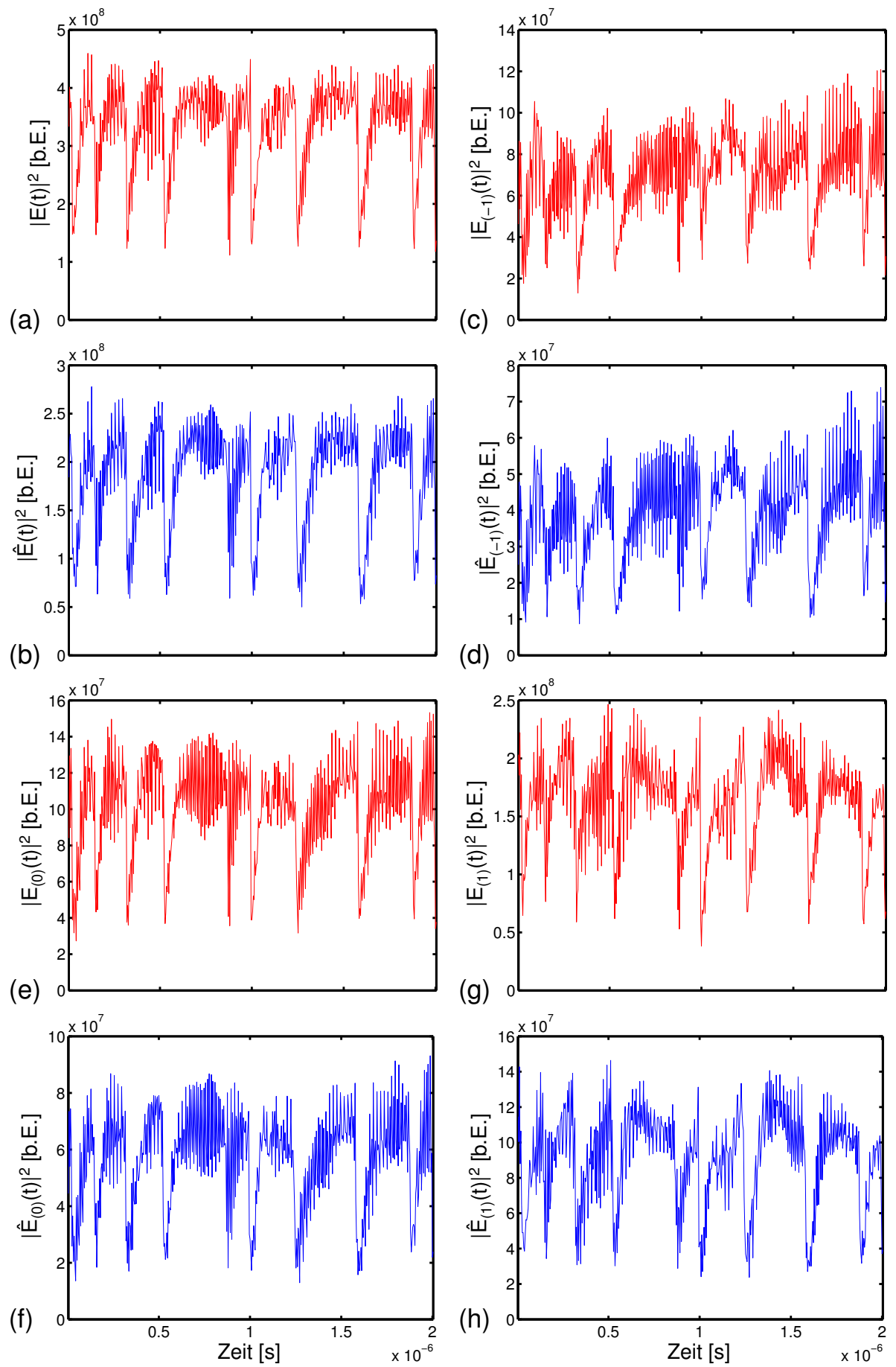

Abbildung 6.14: Gesamtintensitäten beider Laser und die Intensitäten der einzelnen Moden bei synchronisierter LFF-Dynamik. (a), (b) Gesamtintensitäten; (c), (d) Intensität 1. Mode; (e), (f) Intensität 2. Mode; (g), (h) Intensität 3. Mode; rot: Intensität des treibenden Lasers, blau: Intensität des empfangenden Lasers. Ohne externen Resonator im Empfangssystem. 

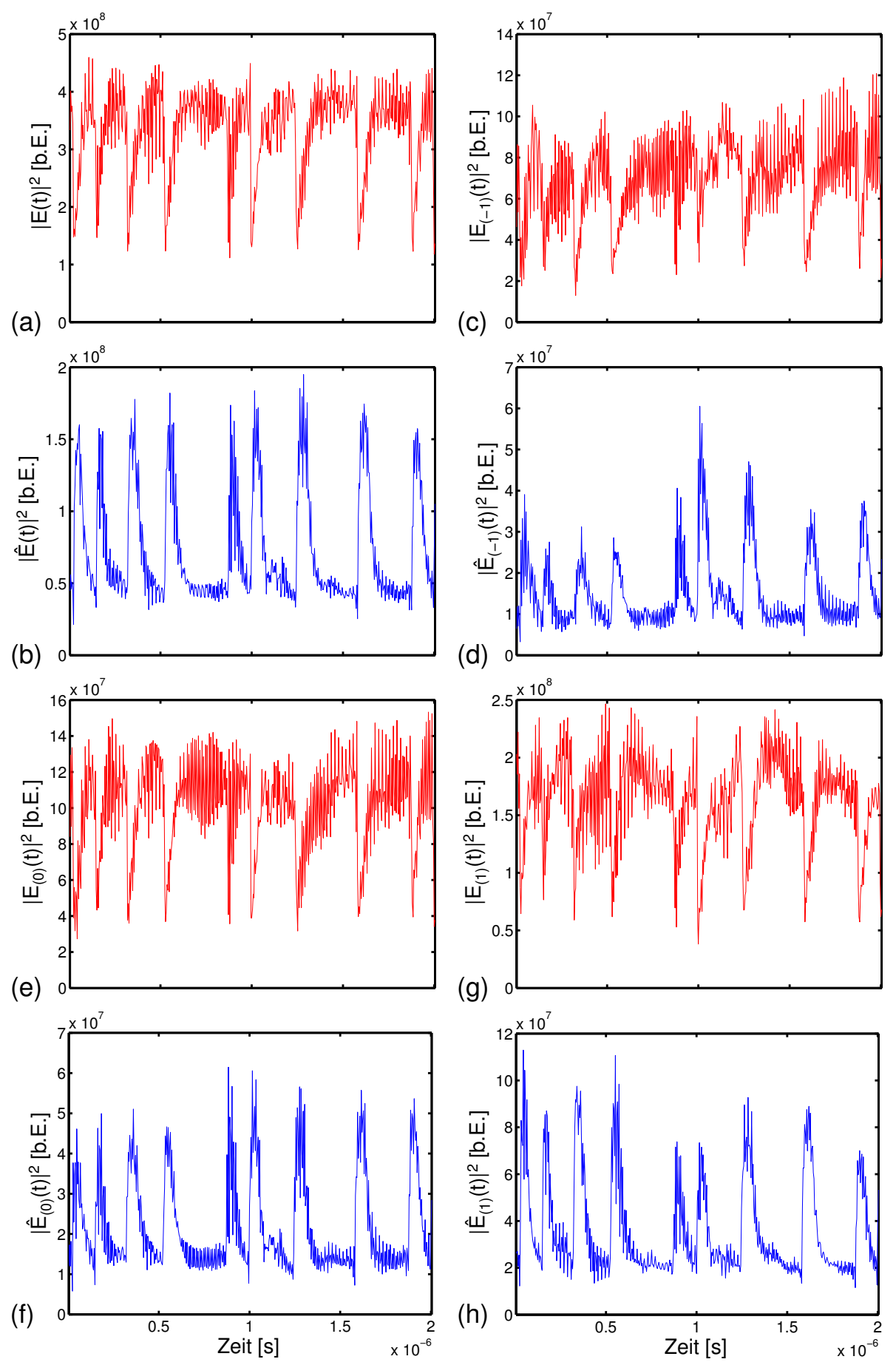

Abbildung 6.15: Gesamtintensitäten beider Laser und die Intensitäten der einzelnen Moden bei antisynchronisierter LFF-Dynamik. (a), (b) Gesamtintensitäten; (c), (d) Intensität 1. Mode; (e), (f) Intensität 2. Mode; (g), (h) Intensität 3. Mode; rot: Intensität des treibenden Lasers, blau: Intensität des empfangende Lasers, ohne externen Resonator im Empfangssystem. 
Da sich die Parameter des treibenden Lasers nicht verändert haben, zeigt sich dasselbe Phänomen auch für die antisynchrone LFJ-Dynamik in Abbildung 6.17. Das experimentell gefundene Phänomen der Modensynchronisation bei Antisynchronisation der Gesamtintensität (vgl. Abbildung 5.32) ist hier gut zu erkennen. Die zweite Mode besitzt hier die höchste Korrelation von $C_{X Y}\left(\tau_{c}\right)=0.78$. Die anderen beiden Moden zeigen eine etwas kleinere Korrelation von $C_{X Y}\left(\tau_{c}\right)=0.64$. Zum Vergleich, die Korrelation der Gesamtintensität beträgt $C_{X Y}\left(\tau_{c}\right)=-0.65$. Dies gilt wohlgemerkt nur für das multimodale Modell. Im monomodalen Fall kann dieses Phänomen nicht auftreten, da hier die Gesamtintensität der (einzigen) Modenintensität entspricht.

Dass die erste und dritte Mode sehr ähnliche Dynamik zeigt und sich zu der zweiten Mode unterscheiden kann, ist schnell einzusehen. Für dieses Multimoden-Modell wurde die in Gleichung (2.32) beschriebene Gewinnfunktion verwendet. Diese Funktion ist symmetrisch, die zweite Mode besitzt die maximale Verstärkung. Die Moden eins und drei besitzen einen etwas kleineren, aber identischen Gewinn.

In Abbildung 6.18 sind die Intensitätsauftragungen für den Kohärenzkollaps gezeigt. Gut zu erkennen ist hier, dass die tiefpassgefilterten Signale der Gesamtintensitäten in den Auftragungen (a) und (b) keine Intensitätseinbrüche zeigen. Dies ist typisch für den Kohärenzkollaps. Eine Korrelation ist für diese Gesamtintensitäten im Experiment durch das verschwindende Signal-Rausch-Verhältnis nicht zu bestimmen. Betrachtet man hingegen die einzelnen Modenintensitäten, so erkennt man gut die Synchronisation der Hüllkurve. Genau dies zeigte die experimentelle Messung in Abbildung 6.18, 

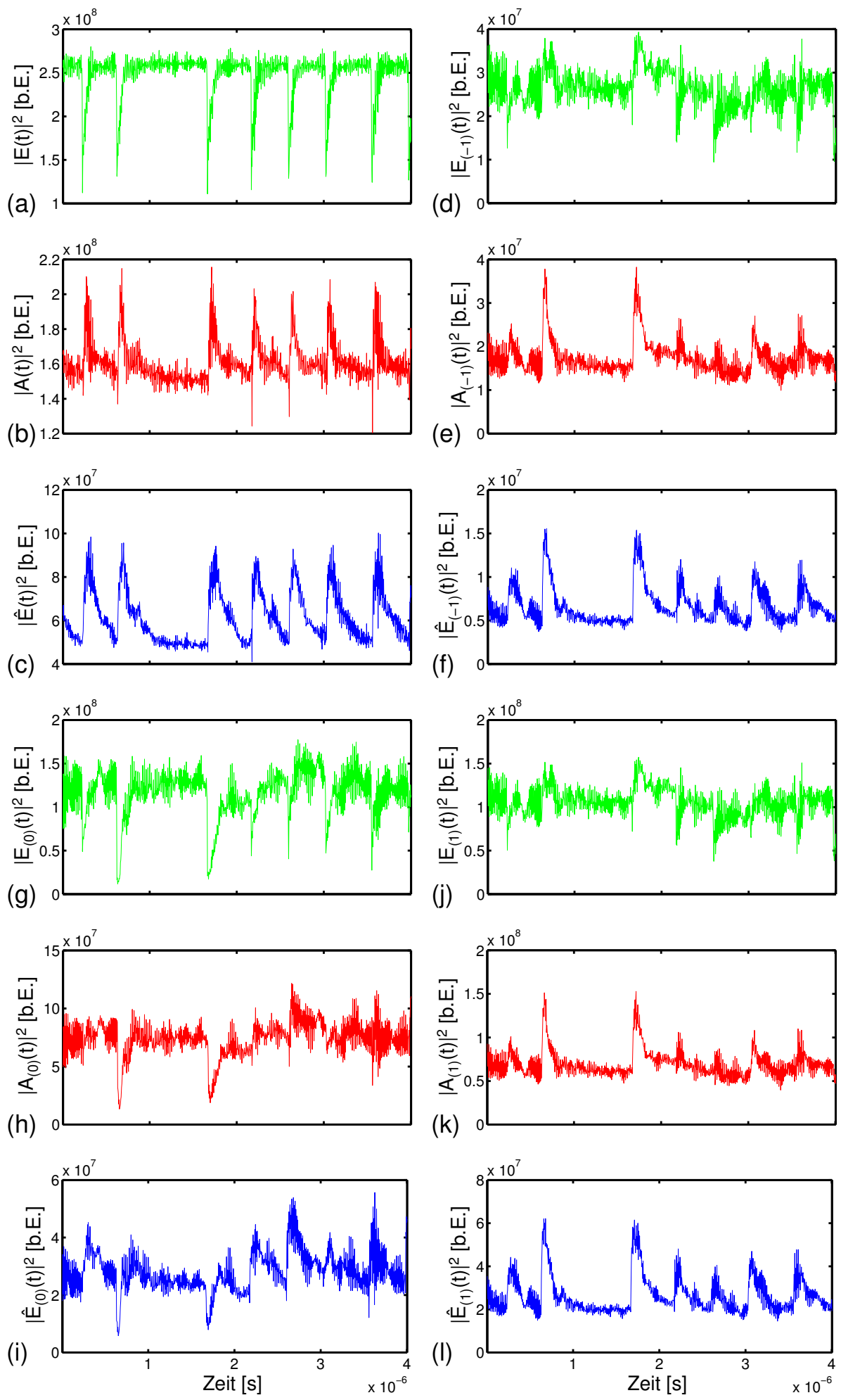

Abbildung 6.16: Gesamtintensitäten beider Laser und die Intensitäten der einzelnen Moden bei synchronisierter LFJ-Dynamik. (a), (b), (c) Gesamtintensitäten; (d), (e), (f) Intensität 1. Mode; (g), (h), (i) Intensität 2. Mode; (j), (k), (l) Intensität 3. Mode; grün: Intensität des Laserfeldes des treibenden Lasers, rot: Intensität des Resonatorfeldes des treibenden Lasers, blau: Intensität des empfangenden Lasers, ohne externen Resonator im Empfangssystem. 

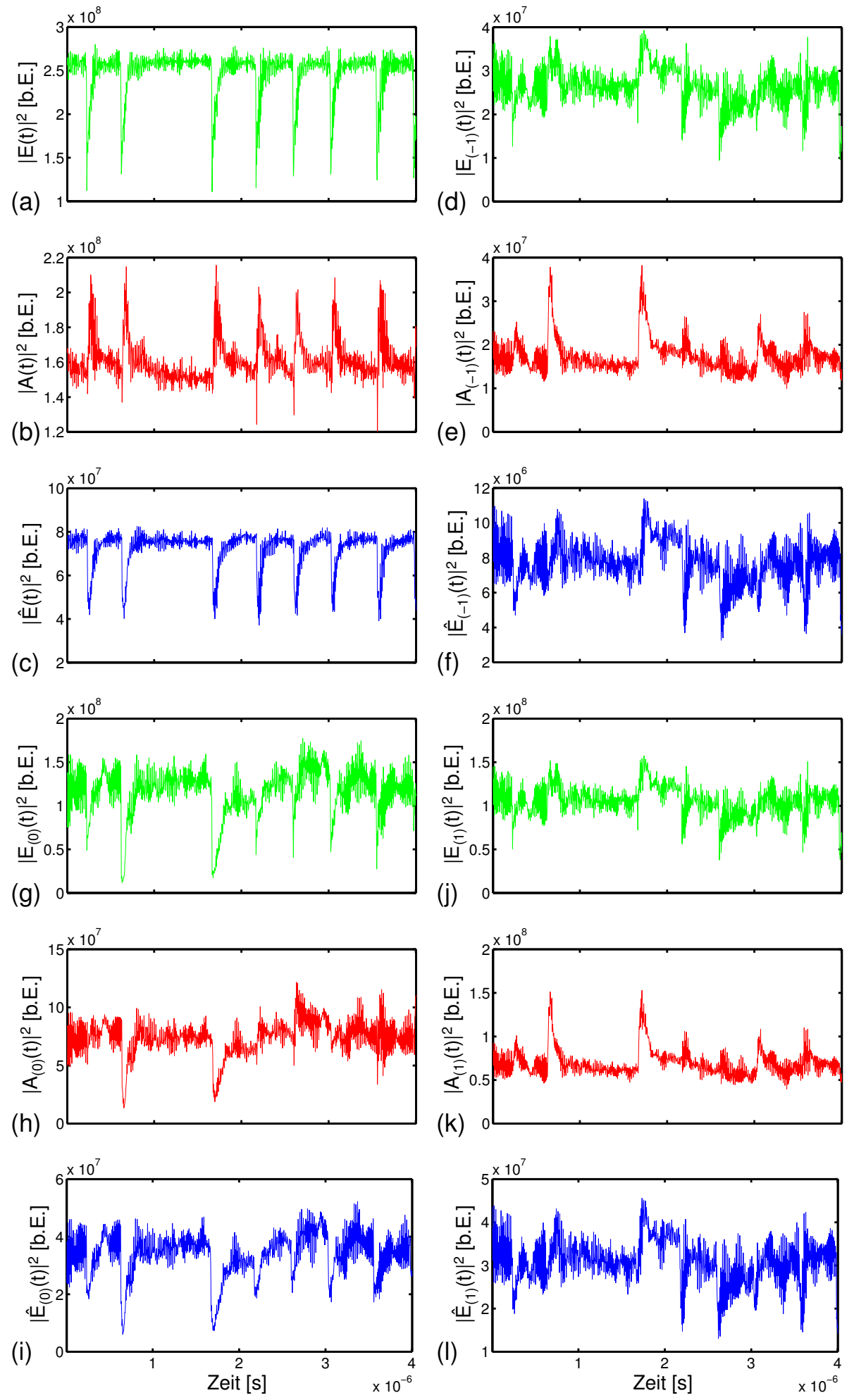

Abbildung 6.17: Gesamtintensitäten beider Laser und die Intensitäten der einzelnen Moden bei antisynchronisierter LFJ-Dynamik. (a), (b), (c) Gesamtintensitäten; (d), (e), (f) Intensität 1. Mode; (g), (h), (i) Intensität 2. Mode; (j), (k), (l) Intensität 3. Mode; grün: Intensität des Laserfeldes des treibenden Lasers, rot: Intensität des Resonatorfeldes des treibenden Lasers, blau: Intensität des empfangenden Lasers, ohne externen Resonator im Empfangssystem. 

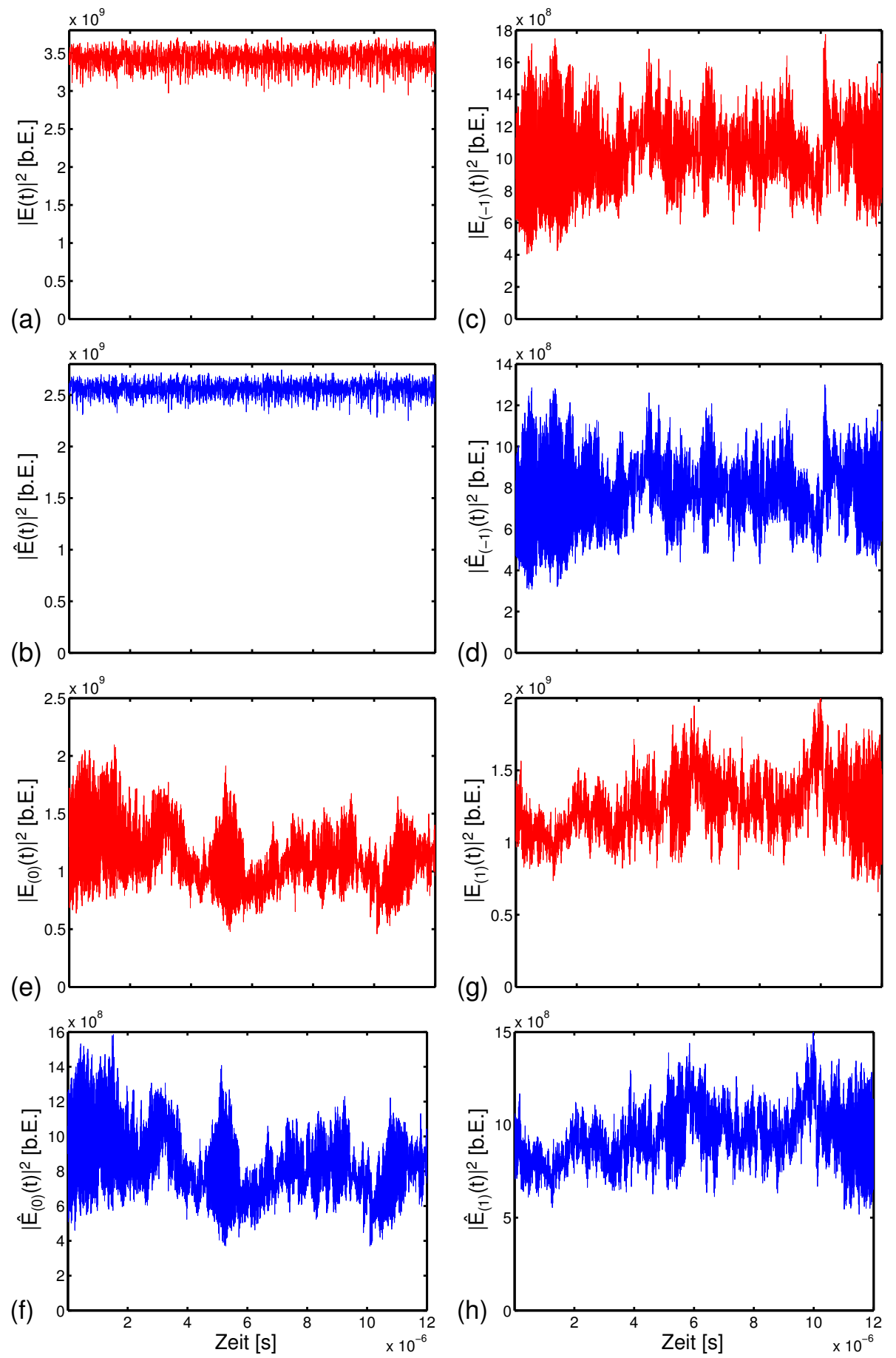

Abbildung 6.18: Gesamtintensitäten beider Laser und die Intensitäten der einzelnen Moden im Kohärenzkollaps. (a), (b) Gesamtintensitäten; (c), (d) Intensität 1. Mode; (e), (f) Intensität 2. Mode; (g), (h) Intensität 3. Mode; rot: Intensität des treibenden Lasers, blau: Intensität des empfangende Lasers, ohne externen Resonator im Empfangssystem. 


\title{
6.7 Puls- und Phasenraumdynamik
}

Die den Intensitätseinbrüchen zugrunde liegende extrem schnelle Pulsdynamik ist im Experiment mit den zur Verfügung stehenden Mitteln nicht messbar. In der Simulation ist es jedoch sehr leicht diese Dynamik zu beobachten. Durch die auf verschiedenen Zeitskalen stattfindende Dynamik ist es jedoch relativ schwierig, eine geeignete Darstellung der Signale zu erreichen. Um diese Problematik nicht noch weiter zu verkomplizieren beschränke ich mich hier auf ein monomodales Modell.

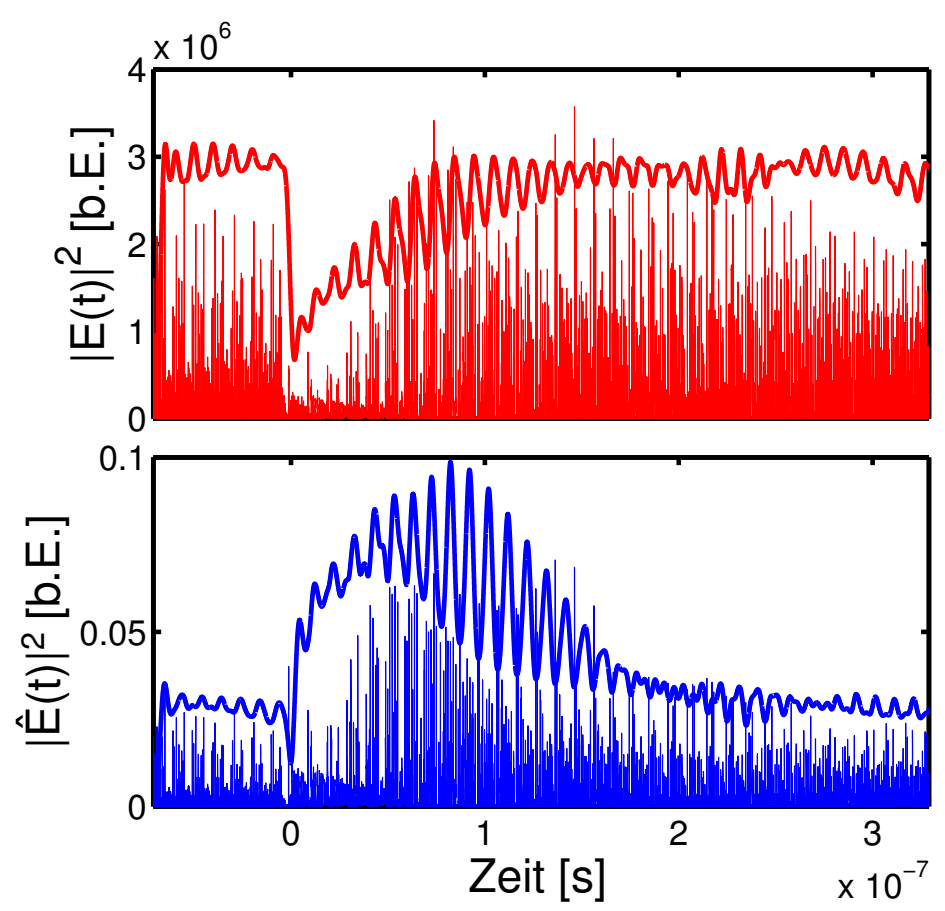

\begin{abstract}
Abbildung 6.19: Intensität der Pulsdynamik des treibenden Lasers (a) und des empfangenden Lasers (b) bei Antisynchronisation des LFF-Dynamik. Die dicke Linie entspricht der tiefpassgefilterte Intensität.
\end{abstract}

Im Falle der LFF-Dynamik ist der synchrone Fall eher unspektakulär, da hier im perfekten Fall auch die Pulsdynamik identisch synchronisiert, wie bereits AHLERs et al. [5] zeigen konnte. Auf eine Darstellung verzichte ich deshalb. Spannender ist der antisynchrone Fall, da es auf Pulsebene keine antisynchrone Dynamik geben kann. Hierzu wären negative Intensitäten notwendig. In Abbildung 6.19] ist diese Pulsdynamik für einen Intensitätseinbruch dargestellt. Diese Zeitskala ist offensichtlich noch nicht klein genug um die Pulse gut aufzulösen. In der feineren Darstellung der Umgebung des Intensitätseinbruch beim Zeitpunkt $t=0 \mathrm{~s}$ in Abbildung 6.20 sind die Pulse recht gut zu erkennen. Die Intensität der Pulse ist vor dem Intensitätseinbruch wesentlich größer als danach. Dies ist beim empfangenden Laser so nicht zu erkennen, die Intensität der 
Pulse bleibt annähernd gleich. Die Pulse entstehen offensichtlich bei beiden Lasern zur selben Zeit (die Kopplungszeit wurde hier der Einfachheit halber auf $\tau_{c}=0$ s gesetzt).

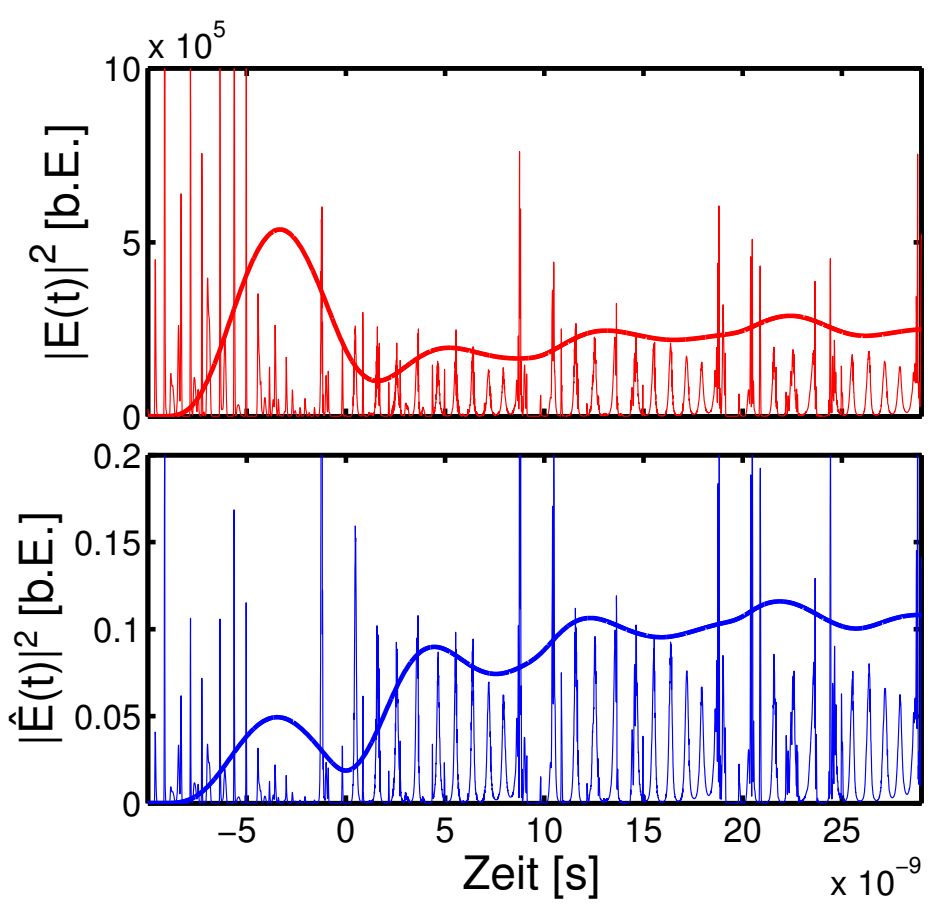

Abbildung 6.20: Pulsdynamik in der Umgebung eines Intensitätseinbruchs des treibenden Laser bei antisynchronisierter LFF-Dynamik.

Einen anderen Einblick in diese Dynamik, die nicht die Intensität sondern die Phase der komplexen Feldamplitude $E(t)$ verwendet, ist mit der Phasenraumdarstellung aus Abschnitt 3.3 möglich. Hierbei wird die Ladungsträgerinversion $N(t)$ gegen die Phasendifferenz $\phi(t)-\phi(t-\tau)$ aufgetragen. Die Ladungsträgerinversion wurde hierbei zur besseren Darstellung tiefpassgefiltert. Der Vorteil dieser Darstellung ist die Einbeziehung der durch die Dynamik entstehende Frequenzverschiebung des Lichtes. In Abbildung 6.21 sind die Trajektorien beider Laser im Phasenraum dargestellt. Die zur Simulation der Antisynchronisation notwendige Frequenzverstimmung $\Delta \omega$ findet sich als Konstante in der Phasendifferenz des empfangenden Lasers wieder. Um eine einheitliche Phasenachse zu bekommen, wurde diese konstante Phasendifferenz von den Phasenwerten des empfangenden Lasers abgezogen. Dadurch stellt die Phasenachse die Kreisfrequenzdifferenz $\omega-\omega_{0}$ beider Laser relativ zur solitären Laserfrequenz $\omega_{0}$ des treibenden Lasers dar.

Das Phasenraumdiagramm sieht für den empfangenden Laser (blaue Kurve) völlig anders aus als für den treibenden Laser (rote Kurve). Die Wanderung der Trajektorie des treibenden Laser entlang der Resonatormoden (ECMs) wird von dem empfangenden 
Laser in gewisser Weise nachvollzogen, wobei sich die Struktur der Trajektorie jedoch deutlich unterscheidet. Die Ladungsträgerdifferenz des Empfangslasers ist kleiner als die des treibenden Laser 6 . Desweiteren erhöht sich bei dem empfangenden Laser im Verlaufe dieser Wanderung die Ladungsträgerinversion leicht. Dadurch sinkt die Intensität im Unterschied zum treibenden Laser. Der vom treibenden Laser ausgeführte Intensitätseinbruch führt die Trajektorie des empfangenden Lasers näherungsweise linear zum Anfang der Wanderung. Die Trajektorie des treibenden Lasers hingegen macht hier einen weiten Bogen im Phasenraum.

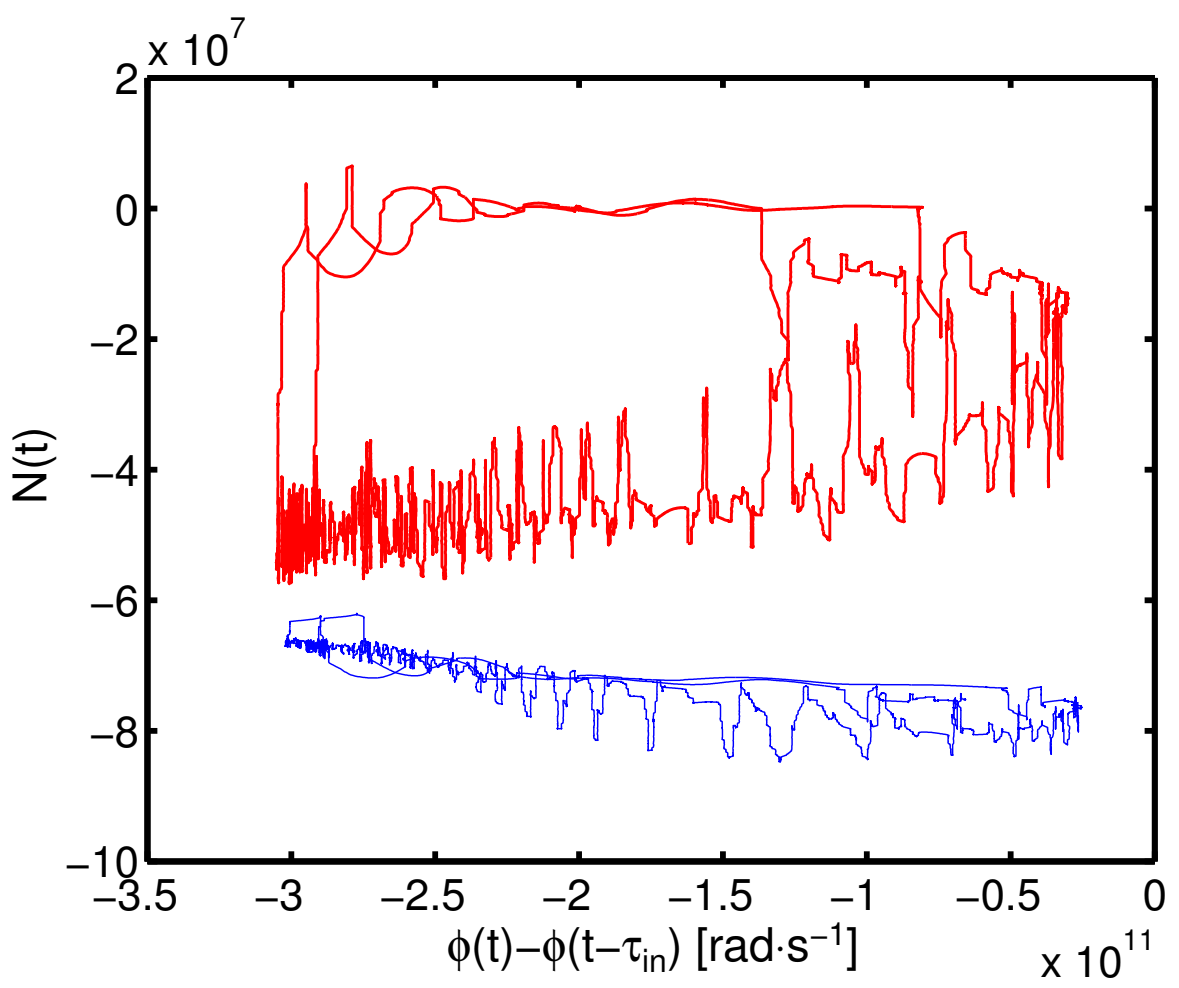

Abbildung 6.21: Phasenraumdarstellung eines Aufschaukelzyklus der Intensität des treibenden Lasers (rot) und Reaktion des Empfangslasers (blau).

Interessant ist hier vor allem die Kreisfrequenz des empfangenden Lasers, sie stimmt praktisch mit dem des treibenden Lasers überein, trotz der modellierten Frequenzverstimmung beider Laser. Dieser Effekt wird sehr gut von den Experimenten gestützt: Zwar können im Experiment die hier dargestellten feinen Frequenzunterschiede der Resonatormoden nicht aufgelöst werden, jedoch ist die vergleichsweise große und gut

\footnotetext{
${ }^{6}$ Normalerweise würde eine kleinere Ladungsträgerdifferenz einem größeren elektrischen Feld entsprechen. Da in diesem Fall jedoch auch das vom treibenden Laser eingekoppelte Licht indirekt auf die Ladungsträgerdifferenz wirkt gilt dies hier so nicht. Die Intensität des empfangenden Lasers ist geringer, wie in der Abbildung [6.19] zu erkennen ist.
} 
detektierbare Frequenzverstimmung beider Laser im antisynchronen Fall nicht auszumachen (u.A. in Auftragung [5.20 gezeigt). Der Empfangslaser wird also auf die Frequenz des treibenden Lasers gezogen.

\section{Phasenraumdynamik der LFJ-Dynamik}

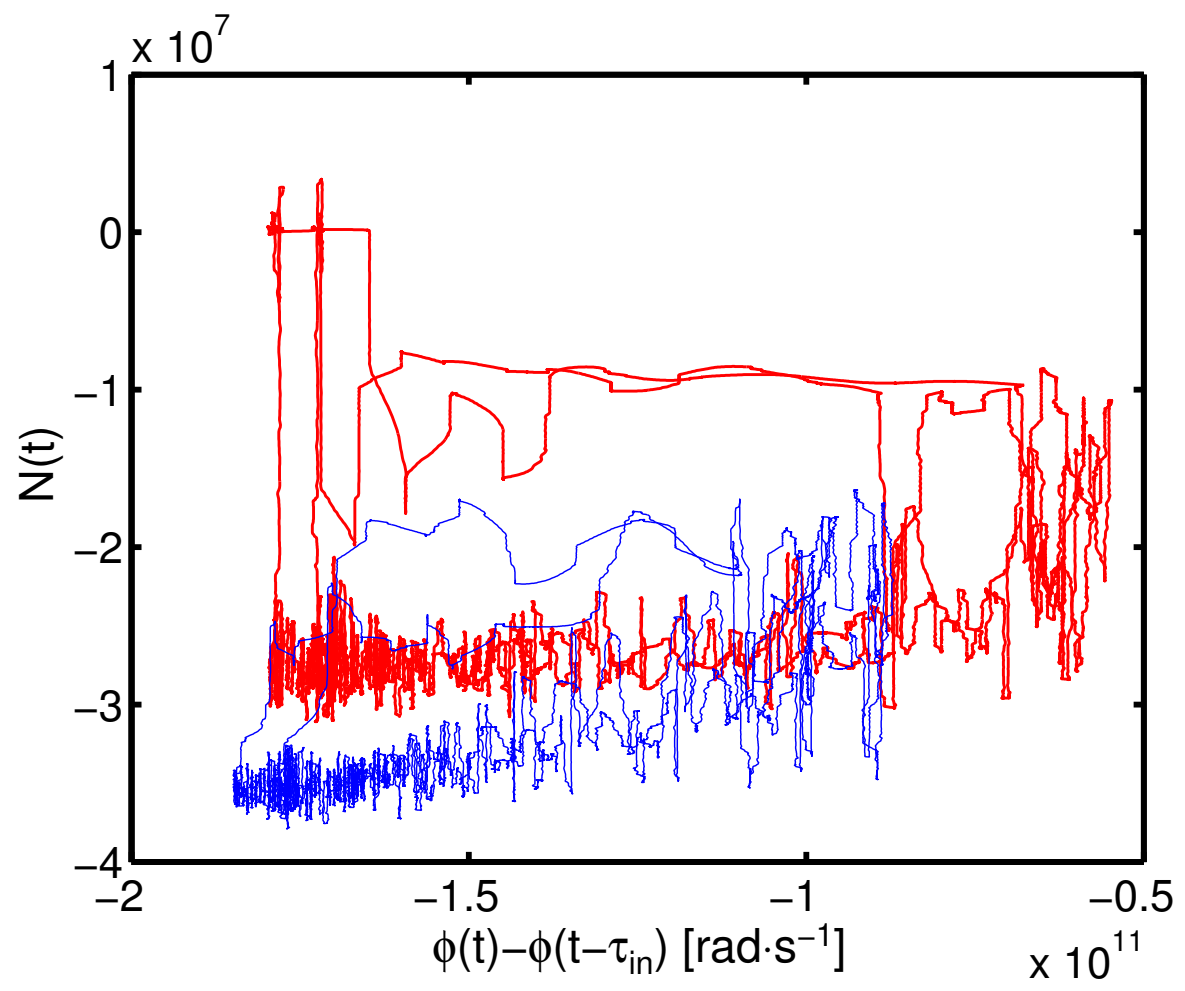

Abbildung 6.22: Phasenraumdynamik bei antisynchronisierter LFJDynamik. Rot: Trajektorie des treibenden Lasers, blau: Trajektorie des empfangenden Lasers.

Die Phasenraumdynamik bei antisynchronisierter LFJ-Dynamik ist in Abbildung 6.22 aufgetragen. Ersteinmal fällt auf, dass sich die Trajektorie des treibenden Systems strukturell nicht wesentlich von der bei LFF-Dynamik unterscheidet. Dies bedeutet, dass sich im Gegensatz zur Intensität die Phase des Resonatorfeldes kaum von der des Laserfeldes unterscheidet, denn nur diese geht in die vorliegende Darstellung ein.

Die Trajektorie des empfangenden Lasers zeigt eine komplett andere Struktur als im Falle der antisynchronisierten LFF-Dynamik und ähnelt entfernt der Trajektorie des treibenden Lasers. Dies ist ein weiteres Indiz für die Vermutung aus Abschnitt 6.5, dass es sich bei dieser antisynchronisierten LFJ-Dynamik eher um die Synchronisation des empfangenden Lasers mit dem Laserfeldes des treibenden Lasers handelt. 


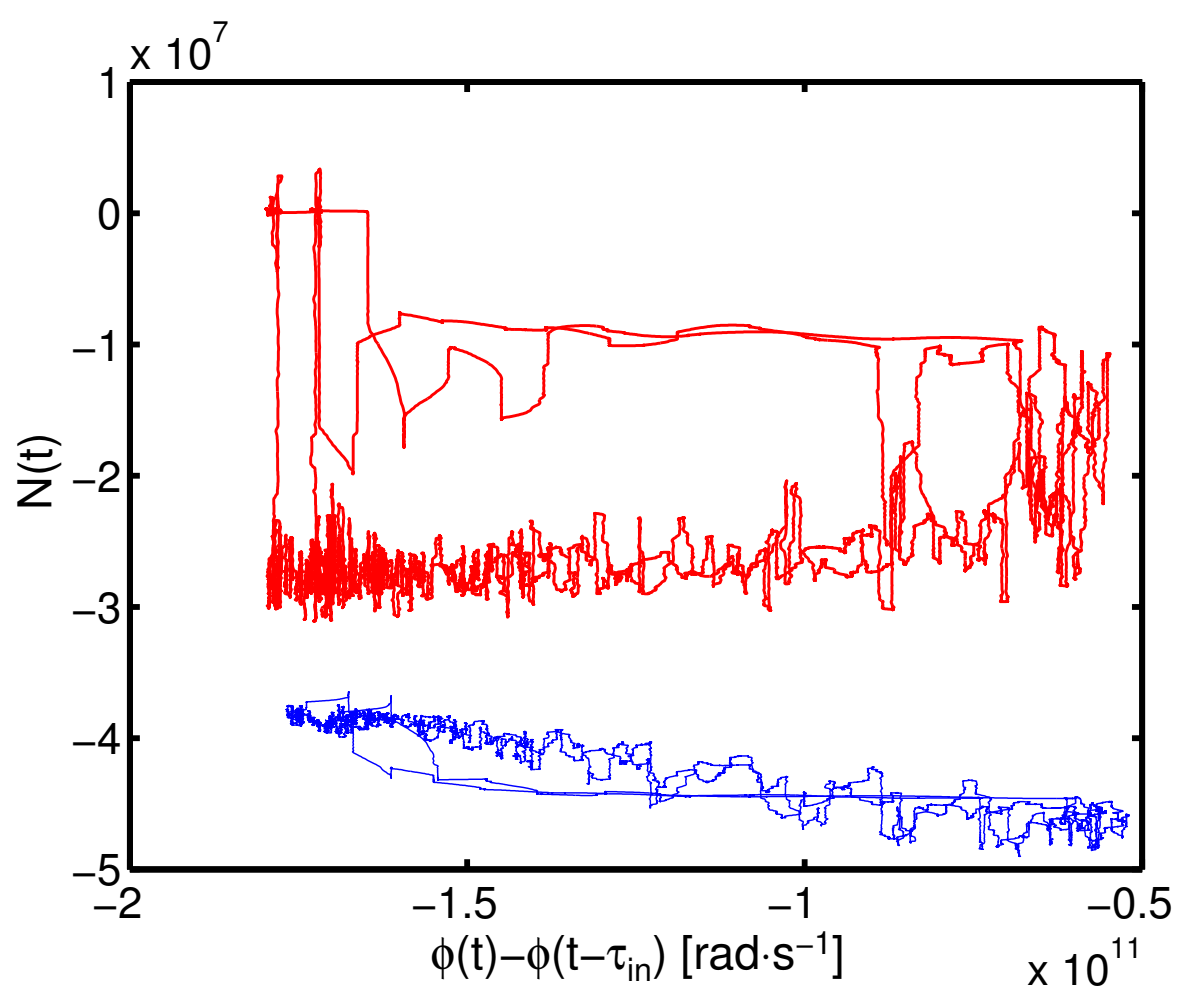

Abbildung 6.23: Phasenraumdynamik bei synchronisierter LFJ-Dynamik. Rot: Trajektorie des treibenden Lasers, blau: Trajektorie des empfangenden Lasers. 
Diese Vermutung würde auch bedeuten, dass die synchronisierte LFJ-Dynamik sinngemäß eine Antisynchronisation des Empfangslasers mit dem Laserfeld des treibenden Lasers darstellen müsste. Es ist dann zu erwarten, dass die Phasenraumtrajektorie des empfangenden Lasers für diesen Fall eine ähnliche Struktur zur Trajektorie des empfangenden Lasers im Falle antisynchronisierte LFF-Dynamik aufweisen müsste. Die Auftragung der Trajektorien für diesen Fall in Abbildung 6.23 bestätigt dies sehr schön. 



\section{Kapitel 7}

\section{Diskussion und Ausblick}

In dieser Arbeit wurde die Synchronisation und Antisynchronisation zweier unidirektional gekoppelter Halbleiterlaser experimentell und numerisch untersucht. Schwerpunkt lag auf der detaillierten Untersuchung der Dynamiken der Intensitätseinbrüche und Intensitätssprünge, die bei optischer Rückkopplung eines Halbleiterlasers auftreten. Für den Fall der Dynamik der Intensitätseinbrüche und der Intensitätssprünge bei optischer Rückkopplung im treibenden Laser zeigte die detaillierte Auftragung des Synchronisationbereiche charakteristische, sich wiederholende Bereiche der Synchronisation mit direkt angrenzenden Bereichen der Antisynchronisation.

Das optische Leistungsspektrum aufgetragen über der Temperatur oder dem Pumpstrom zeigte eine außergewöhnliche Struktur (Abb. 17.1) von systematischen Modensprüngen, die zwischen beiden Lasern synchron ausgeführt wurden. Durch diese Modensprünge ändert sich die Modenfrequenz diskret in einem charakteristischen Raster. Durch die langsame Frequenzänderung entlang der blauen Linie in Abbildung 7.1 konnte in Abschnitt 5.3.4 ein Mechanismus beschrieben werden, der das wiederholte Auftreten der Synchronisations- und Antisynchronisationsbereiche erklären kann.

Durch die Verwendung eines Monochromators konnten die Intensitäten der einzelnen longitudinalen Moden beider Laser aufgezeichnet und auf Synchronisation untersucht werden. Es stellte sich heraus, dass im Falle optischer Rückkopplung nur (identische) Modensynchronisation detektierbar war. Für den Fall der Dynamik der Intensitätseinbrüche im treibenden Laser zeigte sich diese nur im Synchronisationsbereich, im Antisynchronisationsbereich war keinerlei Modenintensität im Empfangslaser auszumachen. Ein unerwartetes Ergebnis zeigte sich bei der Dynamik der Intensitätssprünge im treibenden Laser: Modensynchronisation war nur im Antisynchronisationsbereich zu detektierten, im Synchronisationsbereich war keine Modenintensität im Empfangslaser zu messen. Innerhalb des Kohärenzkollapses, bei dem die Synchronität der Gesamtintensität aufgrund der begrenzten Bandbreite nicht sinnvoll messbar war, zeigte sich 
ebenfalls Modensynchronisation. Spontane Modensprünge, die ohne optische Rückkopplung in den Lasern bei bestimmten Parameterkombinationen stattfinden, konnten zwischen beiden Laser sowohl synchronisiert als auch antisynchronisiert werden.

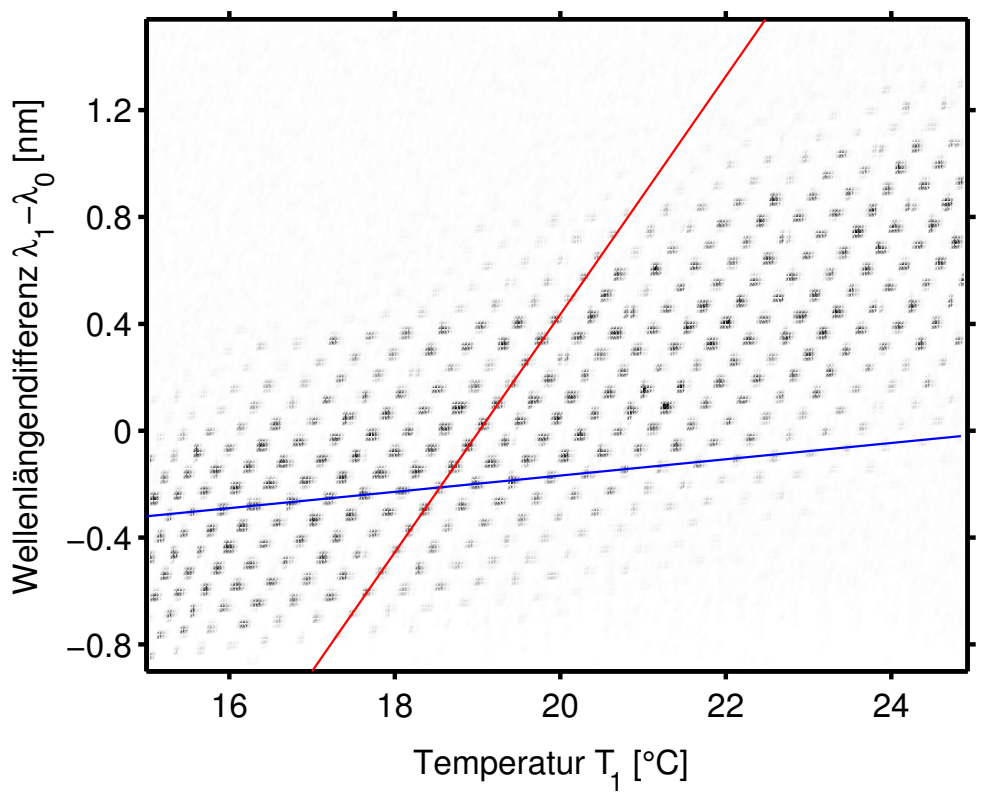

Abbildung 7.1: Systematische Modesprünge des optisch rückgekoppelten, treibenden Lasers bei Variation der Temperatur $T_{1}$.

Als Grundlage für ein numerisches Modell wurde das Lang-Kobayashi-Modell beschrieben. Erweiterungen dieses Modells auf mehrere Moden sowie die Einführung einer Frequenzverstimmung zwischen beiden gekoppelten Lasern zeigte qualitativ gute Übereinstimmung mit den experimentell gefundenen Ergebnissen. Eine Erweiterung des LK-Modell um Mehrfachreflexionen im externen Resonator konnte qualitativ sehr gut die Dynamik der Intensitätssprünge wiedergeben. In diesem Modell zeigt sich auch die von DE TOMASI et al. [15] beschriebene Asymmetrie zwischen den beiden Laserfacetten: die dem externen Resonator zugewandte Laserfacette zeigt Intensitätssprünge, die andere Laserfacette zeigt weiterhin Intensitätseinbrüche.

Die im Experiment auftretende Änderung der Lichtfrequenz durch die Parameter Temperatur und Pumpstrom wurde künstlich in Form einer Frequenzverstimmung beider Laser modelliert. Wird für die Variation der Temperatur des treibenden Lasers näherungsweise eine lineare Frequenzvariation gemäß der roten Linie in Abbildung 7.1 verwendet, so stimmt die Abfolge als auch die Form der Synchronisations- und Antisynchronisationsbereiche in der numerischen Simulation überraschend gut mit den experimentellen Ergebnissen überein. Dies gilt sowohl für die Dynamik der Intensitätseinbrüche als auch die der Intensitätssprünge. 
Die genaue Untersuchung der einzelnen Moden in den numerischen Rechnungen bestätigte noch weitere im Experiment aufgetretenden Effekte. Es konnte z.B. die Synchronisation der Modenintensitäten bei gleichzeitiger Antisynchronisation der Gesamtintensitat für den Fall von Intensitätssprüngen im treibenden Laser beschreiben. Sie lieferte auch weitere Indizien für die Vermutung, dass es sich bei der Antisynchronisation der Intensitätssprünge eher um eine Synchronisation der zeitgleich im treibenden Laser stattfindenden Intensitätseinbrüche handelt.

\section{Ausblick}

Einige der experimentell entdeckten Synchronisationsphänomene sind mit dem hier vorgestellten Modell nicht direkt zu simulieren. Dazu zählen sowohl die systematisch auftretenden Modensprünge bei optischer Rückkopplung als auch die spontanen Modensprünge ohne optische Rückkopplung. Eine sehr faszinierende aber auch sehr anspruchsvolle Aufgabe wäre die vollstände raumzeitliche Simulation beider Laser. Bisher nicht aufgeklärt werden konnte die außergewöhnliche Struktur des Modenspektrums des treibenden Lasers, speziell der systematischen Modensprünge. Eine Struktur, wie sie in Abbildung 7.1] dargestellt ist, würde bei der Annahme eines FabryPerot-Laserresonator nicht entstehen können. Erst die Kopplung zweier Resonatoren des Fabry-Perot-Typs kann eine solche Struktur zeigen. Der externe Resonator hat auf diese Struktur keinen Einfluss, seine Modenabstand ist wesentlich geringer.

Die Dynamik der Intensitätssprünge konnte in den hier präsentierten Experimenten nur an der dem externen Resonator zugewandten Laserfacette beobachtet werden. Das numerische Modell beschreibt das mit den hier detektierbaren Intensitätssprüngen zeitgleiche Auftreten von Intensitätseinbrüchen an der nicht dem externen Resonator zugewandten Laserfacette. Überprüft werden konnte dies im Experiment bisher nicht, da diese Laserfacette bei dem verwendeten Lasertyp nicht zugänglich ist.

Um die numerischen und experimentellen Ergebnisse vergleichen zu können, war es nötig, den Einkopplungsfaktor $\kappa$ unabhängig von der Reflektivität des Spiegels des externen Resonators zu wählen. Möglicherweise ist eine konsistente Wahl dieses Einkopplungsfaktors gemäß der Gleichung (3.2) durch weitere Optimierung der vielen Parameter dieses Modells erreichbar. 



\section{Anhang A}

\section{Symbolverzeichnis}

$\mathcal{E}$

$\mathcal{H}$

$\mathcal{D}$

$\mathcal{B}$

$\mathcal{J}$

$\rho_{f}$

$\epsilon_{0}$

$\mathcal{P}$

$\mu_{0}$

$\sigma$

$\boldsymbol{E}$

$\boldsymbol{P}$

$c$

$\omega_{0}$

$\omega$

$\chi$

$k_{0}$

$\frac{1}{\tau_{p}}$

G

$G_{N}$

$\alpha$

$E(t), E_{(m)}(t)$

$N(t)$

$N_{t r}$

$D$

$J$
Raumzeitliches elektrisches Feld

Raumzeitliches magnetisches Feld

elektrische Flussdichte

magnetische Flussdichte

Stromdichte

Ladungsdichte

Vakuumpermittivität

Polarisation des Mediums

Vakuumpermeabilität

Leitfähigkeit des Mediums

räumliche, komplexe Amplitude des zeitkonstanten elektrisches Feld

räumliche, komplexe Amplitude der zeitkonstanten Polarisation

Lichtgeschwindigkeit im Vakuum

Kreisfrequenz der Resonatormode

Kreisfrequenz des Lasers

komplexe Suszeptibilität

Wellenzahl

optische Verlustrate

Gewinnfunktion des laseraktiven Mediums

differentielle Gewinn des Mediums

Linienbreitenvergrößerungsfaktor

komplexe Amplitude des elektrischen Feldes einer Mode

Ladungsträgerinversion

Transparenzladungsträgerzahl

Konstante der Ladungsträgerdiffusion

Pumpstrom 


\begin{tabular}{|c|c|}
\hline$J_{t h}$ & Pumpstrom der Laserschwelle \\
\hline$p, \hat{p}$ & $\begin{array}{l}\text { Normierter Pumpstrom des treibenden bzw. empfangenden La- } \\
\text { sers }\end{array}$ \\
\hline$\gamma$ & Verlustrate der Ladungsträger \\
\hline$\kappa, \hat{\kappa}$ & Rückkoppelrate \\
\hline$\tau_{\text {in }}$ & Umlaufzeit im internen Resonator \\
\hline$\tau$ & $\begin{array}{l}\text { Umlaufzeit im externen Resonator, bei Kreuzkorrelation Ver- } \\
\text { schiebungszeit }\end{array}$ \\
\hline$r_{2}$ & Reflektivität der rechten Laserfacette \\
\hline$r_{3}$ & Reflektivität des variablen Strahlteilers M1 \\
\hline$E_{0}(t)$ & reele Amplitude der komplexen Amplitude $E(t)$ \\
\hline$\phi(t)$ & Phase der komplexen Amplitude $E(t)$ \\
\hline$A(t)$ & komplexe Amplitude des externen Resonatorfeldes \\
\hline$C_{X Y}$ & normierte Kreuzkorrelationsfunktion \\
\hline$\left|E_{1}(t)\right|^{2},\left|E_{2}(t)\right|^{2}$ & $\begin{array}{l}\text { experimentell aufgenommene Intensitäten des treibenden bzw. } \\
\text { des empfangenden Lasers }\end{array}$ \\
\hline$I_{1}, I_{2}$ & $\begin{array}{l}\text { experimentell eingestellter Pumpstrom des treibenden bzw. emp- } \\
\text { fangenden Lasers }\end{array}$ \\
\hline$T_{1}, T_{2}$ & $\begin{array}{l}\text { experimentell eingestellte Temperatur des treibenden bzw. emp- } \\
\text { fangenden Lasers }\end{array}$ \\
\hline$\lambda_{1}, \lambda_{2}$ & Wellenlänge des treibenden bzw. des empfangenden Lasers \\
\hline$\lambda$ & des Mon \\
\hline$\rho$ & sstärke \\
\hline$\tau_{c}$ & Kopplungsverzögerung \\
\hline$S$ & ationsindex \\
\hline$\Delta \omega_{T}$ & Temperaturkoeffizient der Frequenzverstimmung \\
\hline$\Delta \omega_{I}$ & Pumpstromkoeffizient der Frequenzverstimmung \\
\hline$T_{1}^{(n)}, T_{2}^{(n)}$ & numerische Temperatur \\
\hline$I_{1}^{(n)}, I_{2}^{(n)}$ & numerischer Pumpstrom \\
\hline$|E(t)|^{2},|\hat{E}(t)|^{2}$ & $\begin{array}{l}\text { numerische Gesamtintensität des treibenden bzw. des empfan- } \\
\text { genden Lasers }\end{array}$ \\
\hline$\left|E_{(m)}(t)\right|^{2},\left|\hat{E}_{(m)}(t)\right|^{2}$ & $\begin{array}{l}\text { numerische Modenintensität der m-ten Mode des treibenden } \\
\text { bzw. empfangenden Lasers }\end{array}$ \\
\hline$|A(t)|^{2},\left|A_{(m)}(t)\right|^{2}$ & $\begin{array}{l}\text { numerische Gesamtintensität bzw. m-te Modenintensität des Re- } \\
\text { sonatorsfeldes des treibenden Lasers }\end{array}$ \\
\hline$\Delta T_{1}^{(n)}, \Delta T_{2}^{(n)}$ & $\begin{array}{l}\text { numerische Temperaturdifferenz des treibenden bzw. des emp- } \\
\text { fangenden Lasers }\end{array}$ \\
\hline
\end{tabular}


\begin{tabular}{l|l}
$\Delta T_{1}, \Delta T_{2}$ & $\begin{array}{l}\text { experimentelle Temperaturdifferenz des treibenden bzw. des } \\
\text { empfangenden Lasers zu einer fest gewählten Referenztempera- } \\
\text { tur }\end{array}$
\end{tabular} 



\section{Anhang B}

\section{Komponenten des experimentellen Aufbaus}

\author{
Laserdiodennetzteile \\ Temperaturregelung \\ LD1, LD2 \\ BS1, BS2, BS3, BS4 \\ G \\ $\mathrm{P}$
}

OD

APD1, APD2, APD3, APD4

M1, M2

Spektrometer

Monochromator

Oszilloskop

Oszilloskop
Konstantstromnetzteil, Eigenbau der Institutswerkstatt, extern über Spannung einstellbar

Eigenbau der Institutswerkstatt, Genauigkeit $0.01^{\circ} \mathrm{C}$, extern über Spannung einstellbar

Laserdiode Mitsubishi ML1412R

Strahlteilerwürfel, nichtpolarisierend, Teilungsverhältnis 50:50, Spindler $\&$ Hoyer

Graukeil 1\%-80\% Transmission, Spindler \& Hoyer

Polarisationsfolie, Spindler \& Hoyer

Faradayisolator Optics for Research OFR IO-635$H P, 94 \%$ Transmission in Durchlaßrichtung, $43 \mathrm{~dB}$ Dämpfung in Sperrichtung

Avalanche Photo Diode mit Verstärker, ACgekoppelt

Variabler Strahlteiler, einstellbarer Reflexionsfaktor 8.5\%-85\%, Jodon VBA-200

Positive Light, CCD-Zeile mit 1024 Pixel, spektrale Auflösung $0.02 \mathrm{~nm}$

Acton Research SpectraPro-275, schrittmotorgesteuerter Monochromator, serielles Schnittstelle, spektrale Auflösung variabel $\geq 0.05 \mathrm{~nm}$

LeCroy LC574AM, 4 Kanäle, analoge Bandbreite $1 \mathrm{GHz}$, maximale Abtastrate 4GS/s, Abtastauflösung 8 Bit, Speichertiefe 2MS, GPIB-Interface

HP Infinium, 4 Kanäle, analoge Bandbreite $1.5 \mathrm{GHz}$, maximale Abtastrate 8GS/s, Abtastauflösung 8 Bit Speichertiefe 64kS, GPIB-Interface 
Oszilloskop

PC

DA-Wandler
Tektronix TDS220, 2 Kanäle, analoge Bandbreite $100 \mathrm{MHz}$, maximale Abtastrate $1 \mathrm{GS} / \mathrm{s}$, Abtastauflösung 8 Bit, Speichertiefe 2500S, GPIB-Interface Windows XP, Agilent VEE Pro, Matlab, GPIBInterface, 2 serielle Schnittstellen

2 Kanäle, serielle Schnittstelle, zur Ansteuerung der Pumpstrom- und Temperaturregelung 


\section{Anhang C Farbtabellen}

Die in vielen Abbildungen verwendeten Farbtabellen sind hier zusammengefasst. Die genauen Werte sind bei diesen Darstellung im Allgemeinen nicht relevant, da sie vor allem von dem Messaufbau, den Detektoren und der Verstärkereinstellung des Oszilloskops abhängen.

\section{Fluktuation}

Bei allen Monochromatormessungen in den Abbildungen 5.22, 5.30, 5.31,5.33, und 5.34 wurde die Fluktuation der Messgröße mit der in Abbildung C.1 gezeigten Farbtabelle kodiert.

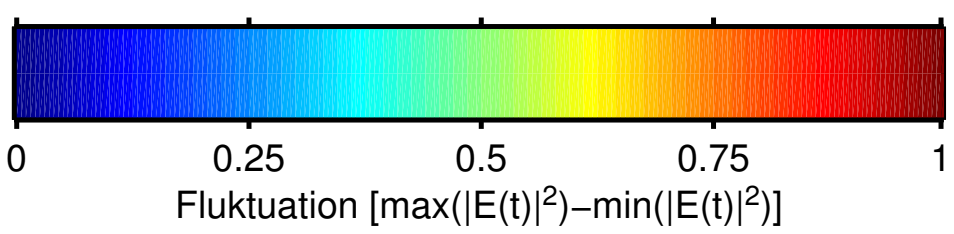

Abbildung C.1: Farbtabelle für die Darstellung der Fluktuationsstärke.

\section{Intensität}

In den Abbildungen 5.16 und 5.17 sowie in 5.26 und 5.27 wurde aufgrund der sehr hohen Auflösung die Intensität mit einem Weiß-Schwarz-Verlauf dargestellt (siehe Abb. C.2). Hierbei steht weiß für keine Intensität und schwarz für die maximal in der Messung vorkommende Intensität $\max \left(|E(t)|^{2}\right)$.

In den geringer aufgelösten Darstellungen der Intensität in Abb. 5.19, 5.20, und 6.1] hat sich die in Abbildung C.3 gezeigte Farbtabelle als sinnvoll erwiesen. 


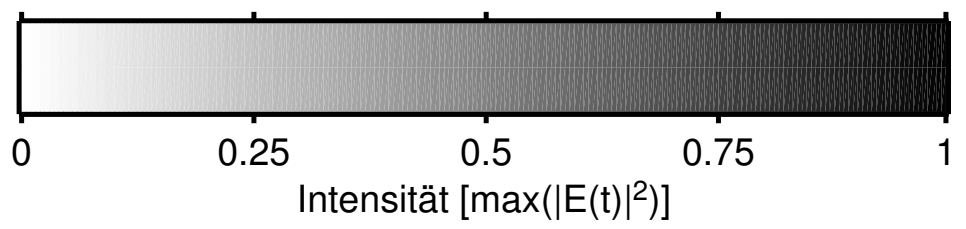

Abbildung C.2: Weiß-Schwarz-Verlauf für die hochaufgelösten Intensitätsdarstellungen.

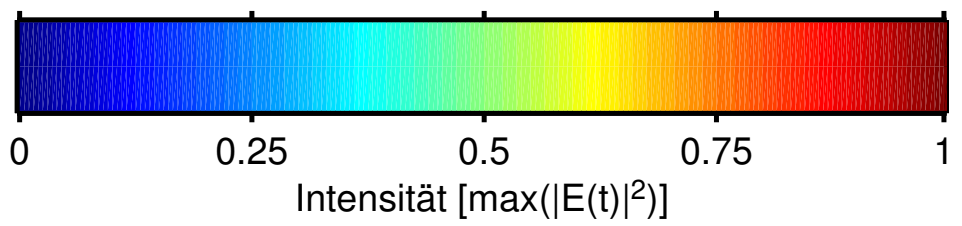

Abbildung C.3: Farbtabelle für die übrigen Intensitätsdarstellung.

\section{Synchronisationsindex}

Die Farbtabelle in Abbildung C.4 wurde für die Darstellung des Synchronisationsindexes verwendet (Abb. 5.4, 5.6, 5.13, 5.14, 5.22, 5.26, 5.27, 5.31, 5.33, 5.34, 6.3, 6.4, 6.5, 6.6. 6.10, 6.11, 6.12, 6.13). Der Wertebereich dieser Farbtabelle geht von $-\max (|S|)$ bis $\max (|S|)$.

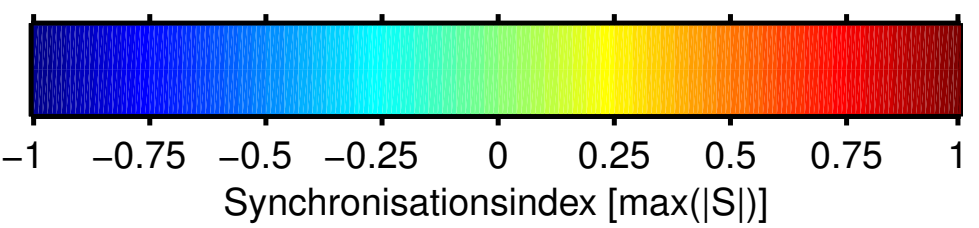

Abbildung C.4: Farbtabelle für die Darstellung des Synchronisationsindexes $S$.

\section{Korrelation}

Für die Darstellung der normierten Korrelation in den Abbildungen [5.3, 5.8 und 5.9] wurde die Farbtabelle aus Abbildung C.5 verwendet. 


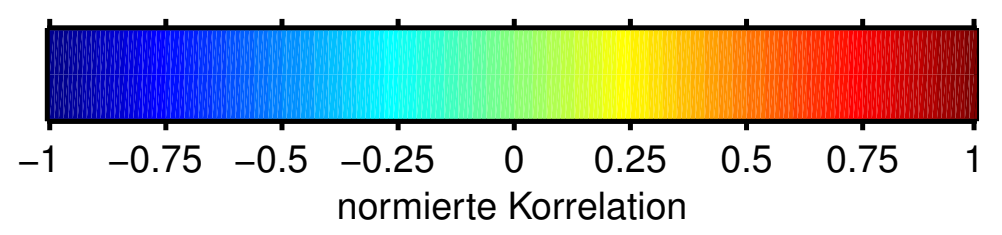

Abbildung C.5: Farbtabelle für die Darstellung der normierten Kreuzkorrelationskoeffizienten $C_{X Y}(\tau)$. 



\section{Anhang D}

\section{Parameter der numerischen Simulation}

\begin{tabular}{l|l}
$r_{2}$ & 0.5 \\
$r_{3}$ & 0.5 für Simulation der LFJ-Dynamik, 0 sonst \\
$\alpha$ & 3.5 \\
Reflexionen & 3 \\
$\frac{1}{\tau_{p}}$ & $7.14 \cdot 10^{11} \mathrm{~s}^{-1}$ \\
$\gamma$ & $5.29 \cdot 10^{8} \mathrm{~s}^{-1}$ \\
$N_{t r}$ & $3.86 \cdot 10^{8}$ \\
$\omega_{0}$ & $2.97 \cdot 10^{15} \mathrm{rad} \cdot \mathrm{s}^{-1}$ \\
$J$ & $3.12 \cdot 10^{17} \mathrm{~s}^{-1}$ \\
$G_{N}$ & $3.5 \cdot 10^{3} \mathrm{~s}^{-1}$ \\
$\kappa$ & $7 \cdot 10^{10} \mathrm{für} \mathrm{LFF}-$ und LFJ-Dynamik \\
$\rho$ & $1 \cdot 10^{11}$ \\
$\Delta \omega_{g}$ & $2.95 \cdot 10^{13} \mathrm{rad} \cdot \mathrm{s}^{-1}$ \\
$\Delta \omega_{L}$ & $7.51 \cdot 10^{11} \mathrm{rad} \cdot \mathrm{s}^{-1}$
\end{tabular}





\section{Literaturverzeichnis}

[1] V. S. Afraimovich, N. N. Verichev und M. I. Rabinovich: Stochastic synchonization of oscillation in dissipativ systems. Radiofizika 29(9), 1050-1060, 1986.

[2] G. P. Agrawal: IEEE J. Quantum Electron. QE-20, 468, 1984.

[3] G. P. Agrawal und N. K. Dutta: Semiconductor Lasers. Van Nostrang Reinhold, 2. Aufl., 1993.

[4] V. Ahlers: Nichtlineare Dynamik und Synchronisation chaotischer Halbleiterlaser mit externen optischen Resonatoren. Diplomarbeit, III. Physikalisches Institut der Georg-August-Universiät zu Göttingen, 1998.

[5] V. Ahlers, U. Parlitz und W. Lauterborn: Hyperchaotic dynamics and synchronisation of external-cavity semiconductor lasers. Phys. Rev. E 58(6), 7208-7213, 1998.

[6] Zh. I. Alferov und R. F. Kazarinov: Semiconductor laser with electric pumping. Inventor's Certificate No. 181737, Application No. 950840 , 1963.

[7] A. P. Bogatov, P. G. Eliseev und B. N. Sverdlov: Anomalous interaction of spectral modes in a semiconductor laser. IEEE J. Quantum Electron. 11(7), 510-515, 1975.

[8] S. Bottani: Synchronization of integrate and fire oscillators with global coupling. Phys. Rev. E 54(3), 2334-2350, 1996.

[9] R. Brown und L. Kocarev: A unified definition of synchronization for dynamical systems. Chaos. 10(2), 344-349, 2000.

[10] L.-Y. Cao und Y.-C. Lai: Antiphase synchronism in chaotic systems. Phys. Rev. E 58, 382-386, 1998.

[11] C. J. Chang-Hasnain, M. Orenstein, A. von Lehmen, L. T. Florez, J. P. Harbison und N. G. Stoffel: Transversal mode characteristics of vertical cavity surfaceemitting lasers. Appl. Phys. Lett. 57(3), 218-220, 1990. 
[12] K. D. Choquette, D. A. Richie und R. E. Leibenguth: Temperature dependence of gain-guided vertical-cavity surface emitting laser polarization. Appl. Phys. Lett. 64(16), 2062-2064, 1994.

[13] J. Cohen und D. Lenstra: Spectral properties of the coherence collapsed state of a semiconductor laser with delayed optical feedback. IEEE J. Quantum Electron. 25(6), 1143-1151, 1989.

[14] J. S. Cohen, F. Wittgrefe, M. D. Hoogerland und J. P. Woerdman: Optical spectra of a semiconductor laser with incoherent optical feedback. IEEE J. Quantum Electron. 26, 982-990, 1990.

[15] F. de Tomasi, E. Cerboneschi und E. Arimondo: Asymmetric pulse shape in the lff instabilities of a semiconductor laser with optical feedback. IEEE J. Quantum Electron. 30(10), 2277-2280, 1994.

[16] C. Degen, B. Krauskopf, G. Jennemann, I. Fischer und W. Elsäßer: Polarization selective symmetry breaking in the near-fields of vertical cavity surface emitting lasers. J. Quantum Semiclass. Opt. 2, 517-525, 2000.

[17] J. E. Epler, S. Gehrsitz, K. H. Gulden, M. Moser, H. C. Sigg und H. W. Lehmann: Mode behavior and high resolution spectra of circularly symmetric GaAs-AlGaAs air-post vertical cavity surface emitting lasers. Appl. Phys. Lett. 69(6), 722, 1996.

[18] J. Faist, F. Capasso, D. L. Sivco, A. L Sirtori, A. L. Hutchinson und A. Y. Cho: Quantum cascade lasers. Science 264, 553, 1994.

[19] I. Fischer, T. Heil, M. Münkel und W. Elsässer: On the mechanism of the lff phenomenon in the coherence collapse of semiconductor lasers: There is hope for sisyphus! In: Physics and Simulation of Optoelectronic Devices IV, herausgegeben von M. Osinski, P. Blood und A. Ishibashi, Bd. 3283 von Proceedings of SPIE, S. 571-579, 1998.

[20] I. Fischer, G. H. M. van Tartwijk, A. M. Levine, W. Elsässer, E. Göbel und D. Lenstra: Fast pulsing and chaotic itinerancy with a drift in the coherence collapse of semiconductor lasers. Phys. Rev. Lett. 76, 220-223, 1996.

[21] T. Fujisaka, H. Yamada: Stability theory of synchronized motion in coupledoscillator systems. Prog. Theor. Phys. 69(1), 32-47, 1983.

[22] T. Fujisaka, H. Yamada: Stability theory of synchronized motion in coupledoscillator systems 2. Prog. Theor. Phys. 70(5), 1240-1248, 1983. 
[23] J. P. Goedgebuer, L. Larger und H. Porte: Optical cryptosystem based on synchronisation of hyperchaos generated by a delayed feedback tunable laser diode. Physical Review Letters 80(10), 2249, März 1998.

[24] J. P. Gordon, H. J. Zeiger und C. H. Townes: The maser - new type of microwave amplifier, frequency standard, and spectrometer. Phys. Rev. 99, 1264, 1955.

[25] M. Grundmann: The present status of quantum dot lasers. Physica E 5(3), 167$184,1999$.

[26] H. Haken: Licht und Materie II: Laser. B. I. Wissenschaftsverlag, Mannheim, Wien, Zürich, 1981.

[27] R. N. Hall, G. E. Fenner, J. D. Kingsley, T. J. Soltys und R. O. Carlson: Coherent light emission from GaAs junctions. Phys. Rev. Lett. 9(9), 366-368, 1962.

[28] W. A. Hamel, M. P. Exter und J. P. Woerdman: Coherence properties of a semiconductor laser with feedback from a distant reflector: Experiment and theory. IEEE J. Quantum Electron. 28(6), 1459-1469, 1992.

[29] T. Heil, I. Fischer und W. Elsäßer: Coexistence of low-frequency fluctuations and stable emission on a single high-gain mode in semiconductor lasers with external optical feedback. Phys. Rev. A 58, 2672-2675, 1998.

[30] C. H. Henry und R. F. Kazarinov: Instability of semiconductor lasers due to optical feedback from distant reflectors. IEEE J. Quantum Electron 22(2), 294, 1986.

[31] N. Jr. Holonyak und S. F. Bevacqua: Appl. Phys. Lett. 1(82), 1962.

[32] G. Huyet, S. Balle, M. Giudici, C. Green, G. Giacomelli und J. R. Tredicce: Low frequency fluctuations and multimode operation of a semiconductor laser with optical feedback. Opt. Comm. 149, 341-347, 1998.

[33] G. Huyet, S. Hegarty, M. Giudici, B. de Bruyn und J. G. McInerney: Statistical properties of the dynamics of semiconductor lasers with optical feedback. Europhys. Lett. 40(6), 619-624, 1997.

[34] C. Huygens: Die Pendeluhr: Horologium oscillatorium (1763). Ostwald's Klassiker der exakten Wissenschaften 192, A. Heckscher und A. v. Öttingen, Leipzig, 1913.

[35] A. Ibaraki, S. Ishikawa, S. Ohkouchi und K. Iga: Pulsed oscillation of GaAlAs/GaAs surface-emitting injection laser. Elec. Lett. 20(10), 420-422, 1984. 
[36] L. Illing, J. Bröcker, L. Kocarev, U. Parlitz und H. D. I. Abarbanel: When are synchronization errors small. Phys. Rev. E 66, 036229, 2002.

[37] J. L Jewell, A. Scherer, S. L. McCall, Y. H. Lee, S. Walker, J. P. Harbison und L. T. Florenz: Low-threshold electrically pumped vertical-cavity surface-emitting microlasers. Elec. Lett. 25(17), 1123-1124, 1989.

[38] C.-M. Kim, S. Rim, W.-H. Kye, J.-W. Ryu und Y.-J. Park: Anti-synchronization of chaotic oscillators. Phys. Lett. A 320, 39-46, 2003.

[39] L. M. Kocarev und U. Parlitz: General approach of chaotic synchronization with applications to communication. Phys. Rev. Let. 74(25), 5028-5031, Juni 1995.

[40] H. Kroemer: A proposed class of heterojunction injection lasers. Proc. IEEE 51, 1782, 1963.

[41] R. Lang und K. Kobayashi: External optical feedback effects on semiconductor injection laser properties. IEEE J. Quantum Electron. QE-16(3), 347-355, 1980.

[42] D. Lenstra, B. Verbeek und A. J. den Boef: Coherence collapse in single-mode semiconductor laser due to optical feedback. IEEE J. Quantum Electron. QE-21, $674,1985$.

[43] A. M. Levine, G. H. M. van Tartwijk, D. Lenstra und T. Erneux: Diode lasers with optical feedback: Stability of the maximum gain mode. Phys. Rev. A 52(5), 3436-3439, 1995.

[44] A. Locquet, F. Rogister, M. Scimanna, P. Megret und M. Blondel: Two types of synchronization in unidirectionally coupled chaotic external-cavity semiconductor lasers. Phys. Rev. E 64, 045203, 2001.

[45] C. Masoller: Anticipation in the synchronization of chaotic semiconductor lasers with optical feedback. Phys. Rev. Lett. 86(13), 2782-2785, 2001.

[46] I. Melngailis: Longitudinal injection-plasma laser of insb. Appl. Phys. Lett. 6(3), 59-60, 1965.

[47] C. R. Mirasso, P. Colet und P. García-Ferández: Synchronization of chaotic semiconductor lasers: Application to encoded communications. IEEE Phot. Technol. Lett. 8, 299-301, 1996.

[48] J. Mørk, B. Tromborg und P. L. Christiansen: Bistability and low-frequency fluctuations in semiconductor lasers with optical feedback. IEEE J. Quantum Electron 24(2), 123-133, 1988. 
[49] J. Mørk, B. Tromborg und J. Mark: Chaos in semiconductor lasers with optical feedback: Theory and experiment. IEEE J. Quantum Electron. 28(1), 93-108, 1992.

[50] Z. Néda, E. Ravasz, Y. Brechet, T. Vicsek und A.-L. Barabási: The sound of many hands clapping. Nature 304, 849, 2000.

[51] M.-W. Pan, B.-P. Shi und G. R. Gray: Semiconductor laser dynamics subject to strong optical feedback. Opt. Lett. 22(3), 166-168, 1997.

[52] E. Patzak, H. Olesen, A. Sugimura und T. Mukai: Electron. Lett. 19, 938, 1983.

[53] L. M. Pecora und T. L. Carroll: Synchronization in chaotic systems. Phys. Rev. Lett. 64(8), 821-824, 1990.

[54] A. Pikovsky, M. Rosenblum und J. Kurths: Synchronization. Cambridge Universty Press, 2001.

[55] A. S. Pikovsky: On the interaction of strange attractors. Z. Phys. B 55, 149-154, 1984.

[56] C. Risch und C. Voumand: Self-pulsation in the output intensity and spectrum of GaAs-AlGaAs cw diode lasers coupled to a frequency-selective external optical cavity. J. Appl. Phys. 48, 2083, 1977.

[57] F. Rogister, P. Megret, O. Deparis und M. Blondel: Coexistance of in-phase and out-of-phase dynamics in a multimode external-cavity laser diode operating in the low-frequency fluctuations regime. Phys. Rev. A 62(061803), 2000.

[58] W. Rong und S. Ke: Inverse synchronization of chaotic systems in an erbiumdoped fibre dual-ring laser using the mutual coupling method. Chin. Phys. 10, 711-715, 2001.

[59] M. G. Rosenblum, A. S. Pikovsky und J. Kurths: Phase synchronisation of chaotic oscillators. Phys. Rev. Lett. 76(11), 1804-1807, 1996.

[60] R. Roy und K. S. Thornburg: Experimental synchronisation of chaotic lasers. Phys. Rev. Lett. 72(13), 2009-2012, 1994.

[61] T. Sano: Antimode dynamics and chaotic itinerancy in the coherence collapse of semiconductor lasers with external feedback. Phys. Rev. A 50(3), 2719-2726, 1994. 
[62] E. M. Shahverdiev, R. A. Nuriev und R. H. Hashimov: Parameter mismatches and inverse synchronization in the ikeda model. Int. J. Modern Phys. B 18(13), 1911-1918, 2004.

[63] S. Sivaprakasam, I. Pierce, P. Rees, P. S. Spencer, K. A. Shore und A. Valle: Inverse synchronization in semiconductor laser diodes. Phys. Rev. A 64, 013805, 2001.

[64] S. Sivaprakasam, E. M. Shahverdiev, P. S. Spencer und K. A. Shore: Experimental demonstration of anticipating synchronization in chaotic semiconductor lasers with optical feedback. Phys. Rev. Lett. 87(154101), 2001.

[65] S. Sivaprakasam und K. A. Shore: Demonstration of optical synchronization of chaotic external-cavity laser diodes. Opt. Lett. 24(7), 466-468, 1999.

[66] H. Soda, K. Iga, C. Kitahara und Suematsu: Gainasp/inp surface emitting injection lasers. Jpn. J. Appl. Phys. 18, 2329-2330, 1979.

[67] P. S. Spencer und K. A. Shore: Multimode iterative analysis of the dynamic and noise properties of laser diodes subject to optical feedback. Quantum. Semiclass. Opt. 9, 819-830, 1997.

[68] T. Sugawara, M. Tachikawa, T. Tsukamoto und T. Shimizu: Observation of synchronization in laser chaos. Phys. Rev. Lett. 72(22), 3502-3505, 1994.

[69] D. W. Sukow, T. Heil, I. Fischer, A. Gavrielides, A. Hohl-AbiChedid und W. Elsäßer: Picosecond intensity statistics of semiconductor lasers operating in the low-frequency fluctuation regime. Phys. Rev. A 60(1), 667-673, 1999.

[70] Y. Takiguchi, H. Fujino und J. Ohtsubo: Experimental synchronization of chaotic oscillations in externally injected semiconductor lasers in a low-frequency fluctuation regime. Opt. Lett. 24(22), 1570-1572, 1999.

[71] M. Tamburrini, P. Spano und S. Piazolla: Appl. Phys. Lett. 43, 410, 1983.

[72] P. Tass, M. G. Rosenblum, J. Weule, J. Kurths, A. Pikovsky, J. Volkmann, A. Schnitzler und H.-J. Freund: Detection of n:m phase locking from noisy data: Application to magnetoencephalography. Phys. Rev. Lett. 81(15), 3291-3294, 1998.

[73] T. Ushio: Chaotic synchronization and controlling chaos based on contraction mappings. Phys. Lett. A 198, 14-22, 1995. 
[74] B. van der Pol: Forced oscillations in a circuit with non-linear resistance. Phil. Mag. 7(3), 65-80, 1927.

[75] J. van der Pol, B. van der Mark: Frequency demultiplication. Nature 120, 363f., 1926.

[76] J. P. van der Ziel, R. Dingle, R. C. Miller, W. Wiegmann und W. A. Nordland Jr.: Laser oscillation from quantum states in very thin $\mathrm{GaAs}^{-} \mathrm{Al}_{0.2} \mathrm{Ga}_{0.8}$ As multilayer structures. Appl. Phys. Lett. 26(8), 463, 1975.

[77] G. H. M. van Tartwijk, A. M. Levine und D. Lenstra: Sisyphus effect in semiconductor lasers with optical feedback. IEEE J. Sel. Topic. Quantum Electron. 1(2), 466-472, 1995.

[78] G. Vaschenko, M. Giudici, J. J. Rocca, C. S. Menoni, J. R. Tredicce und S. Balle: Temporal dynamics of semiconductor lasers with optical feedback. Phys. Rev. Lett. 81(25), 5536-5539, 1998.

[79] E. A. Viktorov und P. Mandel: Low frequency fluctuations in a multimode semiconductor laser with optical feedback. Phys. Rev. Lett. 85(15), 3157-3160, 2000.

[80] I. Wedekind: Experimentelle Untersuchung zur Synchronisation chaotischer Halbleiterlaser. Diplomarbeit, III. Physikalisches Institut Universität Göttingen, 2000.

[81] I. Wedekind und U. Parlitz: Experimental observation of synchronization and anti-synchronization of chaotic low-frequency-fluctuations in external cavity semiconductor lasers. Int. J. Bifurc. Chaos 11(4), 2001.

[82] I. Wedekind und U. Parlitz: Experimental opservation of synchronization and anti-synchronization of chaotic low-frequency- fluctuations in external cavity semiconductor lasers. In: Experimental Chaos, herausgegeben von S. Bocaletti, B. J. Gluckman, J. Kurths, L. M. Pecora und M. L. Spano, S. 335-340, 2002.

[83] I. Wedekind und U. Parlitz: Synchronization and antisynchronization of chaotic power drop-outs and jump-ups of coupled semiconductor lasers. Phys. Rev. E 66, 026218, 2002.

[84] I. Wedekind und U. Parlitz: Experimental observation of mode synchronization of external cavity semiconductor lasers. in Vorbereitung , 2005.

[85] C. E. Wieman und L. Hollberg: Using diode laser for atomic physics. Rev. of Sci. Inst. 62, 1-19, 1991. 
[86] H. G. Winful und L. Rahman: Synchronized chaos and spatiotemporal chaos in arrays of coupled lasers. Phys. Rev. Lett. 65(13), 1575-1578, 1990. 


\section{Danksagung}

Ich möchte mich bei Herrn Prof. Dr. Ulrich Parlitz und Herrn Prof. Dr. Werner Lauterborn herzlich für die sehr gute Betreuung und Ermöglichung dieser Arbeit bedanken.

Großen Dank schulde ich auch den Werkstätten, hier speziell Herrn Dr. Lautscham und Herrn Ebrecht, für die unzähligen Tipps, Reparaturen und Schaltungsänderungen bestehender elektronischer Geräte, sowie Neubau diverser Pumpstrom- und Temperaturregelungen. Für die unzähligen Umbauten der Laserdiodenhalterungen schulde ich neben Herrn Ebrecht für die elektrische Seite auch Herrn Hille aus der mechanischen Werkstatt meinen Dank. Herrn Geiling danke ich sehr für den nervenzehrenden Kampf mit der anfangs sehr instabilen Workstation- und Laborrechnerhardware.

Ein weiteres Dankeschön geht an die Mitglieder der Arbeitsgruppe Nichtlineare Dyna$m i k$. Trotz meines „Einsiedlerthemas“ - niemand weiteres beschäftigte sich in diesem Themenbereich - konnte ich doch von vielen Tipps und Anregungen profitieren. Speziell möchte ich Dr. Reinhard Geisler für die vielen Tipps rund um die Themen Agilent VEE Pro, experimentelle Optik und Gerätetechnik danken. Auch Dipl.-Phys. David Engster und Dipl.-Phys. Jörg Dittmar danke ich für die unzähligen Tipps rund um Matlab sehr. Bei Dipl.-Phys. Alexander Hornstein als meinem Zimmernachbar und Dipl.-Phys. Matthias Jüschke bedanke ich mich für die vielen kleinen und großen Hilfen und vor allem für das Aushalten meines redseligen Gemütes.

Bei unserer Fotografin und den Angestellten möchte ich mich für die freundliche Hilfe bei vielen kleinen und großen Problemen bedanken.

Allen Institutsangehörigen danke ich für die freundliche und offene Atmosphäre. 



\section{Lebenslauf}

\section{Persönliche Daten}

\begin{tabular}{l|l} 
Name & Immo Wedekind \\
Geburtsdatum & 05.12 .1974 \\
Geburtsort & Köln \\
Eltern & Angelika und Dr. Klaus Wedekind \\
Staatsangehörigkeit & deutsch \\
Familienstand & ledig
\end{tabular}

\section{Schulausbildung}

$1981-1985$

$1985-1987$

1987-1994

1994
Grundschule Hasenburger Berg, Lüneburg Orientierungsstufe Oedeme, Lüneburg Gymasialzweig der Fritz-Reuter-Schule, Bad Bevensen Abitur

\section{Wissenschaftliche Ausbildung}

WS1995/96-SS1997

Oktober 1997

WS1997/98-SS2000

November 2000

$11 / 2000-4 / 2001$

$5 / 2001-10 / 2004$

WS2001/02-SS2002

WS2002/03-

WS2004/05
Grundstudium Physik an der Georg-August-Universität zu Göttingen

Physikvordiplom

Hauptstudium Physik an der Georg-August-Universität

Diplomprüfung Physik

wissenschaftliche Hilfskraft am Dritten Physikalischen Institut der Universität Göttingen

Wissenschaftlicher Mitarbeiter am Dritten Physikalischen Institut der Universität Göttingen

Aufbaustudiengang Physik an der Georg-August-Universität zu Göttingen

Promotionsstudiengang Physik an der Georg-August-Universität zu Göttingen 